\title{
Guía de Práctica Clínica de Anticoncepción Hormonal e Intrauterina
}





\section{Guía de Práctica Clínica de Anticoncepción Hormonal e Intrauterina}

GUÍAS DE PRÁCTICA CLÍNICA EN EL SNS

MINISTERIO DE SANIDAD, CONSUMO Y BIENESTAR SOCIAL 
Esta GPC es una ayuda a la toma de decisiones en la atención sanitaria. No es de obligado cumplimento ni sustituye el juicio clínico del personal sanitario.

\section{Edición:}

Edita: Ministerio de Sanidad, Consumo y Bienestar Social

Edita: Instituto Aragonés de Ciencias de la Salud (IACS)

NIPO: 731-18-013-4

Maquetación: ARPIrelieve, S. A. 
"Este documento se ha realizado al amparo del convenio de colaboración suscrito por el Instituto de Salud Carlos III, organismo autónomo del Ministerio Economía y Competitividad, y el Instituto Aragonés de Ciencias de la Salud, en el marco del desarrollo de actividades de la Red Española de Agencias de Evaluación de Tecnologías Sanitarias y Prestaciones del SNS, financiadas por el Ministerio de Sanidad, Consumo y Binestar Social".

Esta guía debe citarse:

Grupo de trabajo de la Guía de Práctica Clínica de Anticoncepción Hormonal e Intrauterina. Guía de Práctica Clínica de Anticoncepción Hormonal e Intrauterina. Ministerio de Sanidad, Consumo y Bienestar Social. Instituto Aragonés de Ciencias de la Salud; 2019. Guías de Práctica Clínica en el SNS. 



\section{Índice}

$\begin{array}{ll}\text { Presentación } & 7\end{array}$

$\begin{array}{ll}\text { Autoría y Colaboraciones } & 9\end{array}$

$\begin{array}{lr}\text { Preguntas para responder } & 13\end{array}$

Clasificación de la calidad de la evidencia y graduación de la fuerza de las recomendaciones

$\begin{array}{lr}\text { Recomendaciones de la GPC } & 19\end{array}$

1. Introducción 25

2. Alcance y objetivos 27

3. Metodología 29

4. Eficacia y seguridad de los métodos anticonceptivos 33

4.1. Anticonceptivos hormonales e intrauterinos 33

4.2. Tromboembolismo 40

5. Anticoncepción de urgencia 51

5.1. Eficacia y seguridad de la anticoncepción de urgencia 51

5.2. Aspectos relacionados con la libre dispensación de la anticoncepción de urgencia 58

6. Manejo clínico de los métodos anticonceptivos 65

6.1. Consideraciones previas 65

6.2. Controles necesarios para el seguimiento de usuarias de anticonceptivos hormonales e intrauterinos 73

6.3. Manejo de las alteraciones del patrón de sangrado en anticoncepción 82

6.4. Eventualidades en el uso de la anticoncepción 91

6.5. Manejo clínico de la anticoncepción intrauterina 102

7. Situaciones específicas 111

7.1. Usuarias adolescentes 111

$\begin{array}{ll}\text { 7.2. Edad reproductiva avanzada } & 123\end{array}$

7.3. Sobrepeso y obesidad 127

$\begin{array}{ll}\text { 7.4. Puerperio } & 134\end{array}$

8. Estrategias diagnósticas y terapeúticas 141

9. Difusión e implementación 147

10. Anexos 149

11. Bibliografía 159 



\section{Presentación}

Documentar la variabilidad de la práctica clínica, analizar sus causas y adoptar estrategias orientadas a eliminarla, han demostrado ser iniciativas que fomentan la toma de decisiones efectivas y seguras, centradas en los pacientes, por parte de los profesionales sanitarios. Entre dichas estrategias destaca la elaboración de Guías de Práctica Clínica (GPC), «conjunto de recomendaciones basadas en una revisión sistemática de la evidencia y en la evaluación de los riesgos y beneficios de las diferentes alternativas, con el objetivo de optimizar la atención sanitaria a los pacientes».

Entre las prioridades del Ministerio de Sanidad, Consumo y Bienestar Social, se encuentra consolidar la elaboración de GPC, coordinada desde GuíaSalud, en el marco de la Red Española de Agencias de Evaluación de Tecnologías Sanitarias y Prestaciones del Sistema Nacional de Salud (SNS).

Es en este contexto en el que se enmarca la presente Guía de Práctica Clínica de Anticoncepción Hormonal e Intrauterina.

Disponer de una GPC en la que se aborda el manejo de la anticoncepción de urgencia, y el manejo clínico de estos métodos anticonceptivos, prestando especial atención a poblaciones específicas, permite ofrecer recomendaciones en términos de eficacia y seguridad, dirigidas a un uso informado de los anticonceptivos, tanto hormonales como intrauterinos.

Esta GPC aborda aspectos específicos, como el seguimiento clínico de las usuarias de anticonceptivos hormonales e intrauterinos, eventualidades en el manejo de la anticoncepción hormonal como el olvido de la toma, o la utilización de estas tecnologías en la edad reproductiva avanzada.

Deseamos que esto conlleve asociado una reducción de la incertidumbre y controversias en la utilización de los métodos anticonceptivos, así como una reducción en la variabilidad de su uso, no justificado por razones sociodemográficas.

Esta guía es el resultado del gran esfuerzo realizado por un grupo de profesionales sanitarios pertenecientes a distintas especialidades y sociedades científicas, y representantes de varias Sociedades Científicas implicadas en la atención de las personas que acuden al Sistema Sanitario solicitando información para planificar su actividad sexual y reproductiva o ante la necesidad de tomar decisiones sobre la elección de un método anticonceptivo.

Desde la Dirección General de Salud Pública, Calidad e Innovación agradecemos a todas estas personas el trabajo realizado y esperamos que pueda ayudar a la población y a los profesionales en la toma de decisiones, mejorando la salud sexual y reproductiva de aquellas personas que desean disponer de mayor información sobre la anticoncepción.

D. ${ }^{a}$ PILAR APARICIO AZCÁRRAGA Directora General de Salud Pública, Calidad e Innovación

La presente Guía de Práctica Clínica responde a preguntas clínicas concernientes a este proceso y se dirige, principalmente, a los profesionales implicados en el manejo de la anticoncepción. 



\section{Autoría y Colaboraciones}

\section{Grupo de trabajo de la GPC de Anticoncepción Hormonal e Intrauterina}

Ma Jesús Alonso Llamazares. Médico especialista en Medicina Familiar y Comunitaria. Centro Provincial de Orientación Sexual para Jóvenes. Málaga.

Lorenzo Arribas Mir. Médico especialista en Medicina Familiar y Comunitaria. Unidad Clínica La Chana. Granada.

Joaquim Calaf Alsina. Médico especialista en Ginecología y Obstetricia. Hospital de la Santa Creu i Sant Pau. Barcelona.

$\mathbf{M}^{\mathbf{a}}$ Jesús Cancelo Hidalgo. Médico especialista en Ginecología y Obstetricia. Hospital General Universitario. Guadalajara.

Esther de la Viuda García. Médico especialista en Ginecología y Obstetricia. Hospital General Universitario. Guadalajara.

Natividad Fidalgo Rodríguez. Matrona y Sexóloga. Hospital Universitario Central de Asturias. Oviedo.

Patricia Gavín Benavent. Médico Especialista en Microbiología y Parasitología. Instituto Aragonés de Ciencias de la Salud (IACS). Zaragoza.

José Vicente González Navarro. Médico especialista en Ginecología y Obstetricia. Hospital Lozano Blesa. Zaragoza.

Paloma Lobo Abascal. Médico especialista en Ginecología y Obstetricia. Hospital Infanta Sofía, San Sebastián de los Reyes. Madrid.

Cristina Martínez Bueno. Matrona. ASSIR Barcelona Ciutat. Institut Català de la Salut. Barcelona.

Francisca Martínez San Andrés. Médico especialista en Ginecología y Obstetricia. Salut de la Dona Dexeus. Barcelona.

Ezequiel Pérez Campos. Médico especialista en Ginecología y Obstetricia. Hospital de Requena. Valencia.

Isabel Ramirez Polo. Médico especialista en Medicina Familiar y Comunitaria. UGC Dr. Cayetano Roldan, San Fernando. Cádiz

Ivan Solà Arnau. Investigador Centro Cochrane Iberoamericano. CIBER Epidemiología y Salud Pública (CIBERESP). Barcelona.

\section{Coordinación}

Juan Ignacio Martín Sánchez. Médico Especialista en Medicina Preventiva y Salud Pública. Instituto Aragonés de Ciencias de la Salud. Zaragoza.

Ivan Solà Arnau. Investigador. Centro Cochrane Iberoamericano, Barcelona. 


\section{Colaboración}

Àngels Avecilla Palau. Médico especialista en Ginecología y Obstetricia. CASSIR Badalona Servicios Asistenciales (Badalona).

David Rigau Comas. Médico especialista en Farmacología Clínica. Centro Cochrane Iberoamericano (Barcelona).

\section{Revisión externa}

Eva Adarve Hidalgo. Matrona. ASSIR Hospital de Mollet. Mollet del Vallès.

José Gutiérrez Ales. Médico especialista en Ginecología y Obstetricia. Departamento de Obstetricia y Ginecología, Hospital Virgen Macarena. Sevilla.

Ma Angeles Gómez Martínez. Médico especialista en Ginecología y Obstetricia. Centro de Salud Sexual y Reproductiva Alicante III. Alicante.

Roberto Lertxundi Barañano. Médico especialista en Salud Sexual y Reproductiva. Clínica Euskalduna. Bilbao.

Inaki Lete Lasa. Médico especialista en Ginecología y Obstetricia. Servicio de Ginecología y Obstetricia, Hospital Universitario Araba. Vitoria Gasteiz.

Hildegard Mausbach Reisen. Médico especialista en Ginecología y Obstetricia. Societat Catalana de Contracepció, ASSIR Mataró. Mataró.

Nicolás Mendoza Ladrón de Guevara. Médico especialista en Ginecología y Obstetricia. Departamento de Obstetricia y Ginecología, Facultad de Medicina, Universidad de Granada. Granada.

Ana Felisa Muñoz Ruiz. Enfermera especialista Obstétrico-Ginecológica (Matrona). Atención Primaria Sector III Zaragoza. C.S. Utebo. Zaragoza.

Amparo Ortega del Moral. Médico especialista en Medicina Familiar y Comunitaria. Grupo de Atención a la Mujer de SEMFYC, Grupo de Atención a la salud reproductiva de las mujeres SAMFYC, Centro de Salud Gran Capitán. Granada.

María Lourdes Ortiz Villanueva. Graduado en Enfermería Especialidad Matrona. Licenciatura Documentación. Doctora. Centro de Salud de Cella. Teruel.

Núria Parera Junyent. Médico especialista en Ginecología y Obstetricia. Salut de la Dona Dexeus. Barcelona.

Macarena Quesada Moreno. Médico especialista en Medicina Familiar y Comunitaria. Centro de Salud Campoamor. Alicante.

Alberto Salamanca Ballesteros. Médico especialista en Ginecología y Obstetricia. Departamento de Obstetricia y Ginecología, Facultad de Medicina, Universidad de Granada. Granada.

María Isabel Serrano Fuster. Médica especialista en Ginecología y Obstetricia. Programa de Salud Sexual y Reproductiva de Madrid-Salud. Ayuntamiento de Madrid. Voluntaria de la FPFE. Madrid. 


\section{Sociedades Colaboradoras}

Miembros de estas sociedades han participado en la autoría, revisión externa de la GPC.

Federación de Asociaciones de Matronas de España

Federación de Planificación Familiar Estatal

Sociedad Española de Contracepción

Sociedad Sociedad Española de Ginecología y Obstetricia

Sociedad Española de Medicina Familiar y Comunitaria

Declaración de interés: Todos los miembros del Grupo de Trabajo, así como las personas que han participado en la revisión externa, han realizado la declaración de interés que se presenta en el Anexo 4. 



\section{Preguntas para responder}

\section{Eficacia y seguridad de los métodos anticonceptivos}

\subsection{Anticonceptivos hormonales}

1. ¿Cuál es la eficacia y efectividad de los anticonceptivos hormonales?

2. ¿Existe alguna diferencia entre los intervalos libres de hormona en la anticoncepción hormonal combinada (24/4 vs $21 / 7)$, en términos de eficacia y efectos adversos?

\subsection{Anticonceptivos intrauterinos}

3. ¿Cuál es la eficacia y efectividad de los anticonceptivos intrauterinos?

\subsection{Tromboembolismo}

4. ¿En qué casos deben realizarse consideraciones especiales en usuarias de anticonceptivos hormonales en relación con el riesgo de tromboembolismo venoso?

5. ¿En qué casos deben realizarse consideraciones especiales en usuarias de anticonceptivos hormonales en relación con el riesgo de tromboembolismo arterial?

\section{Anticoncepción de urgencia}

2.1. Eficacia y seguridad de la anticoncepción de urgencia

6. ¿Cuál es la eficacia de los anticonceptivos de urgencia?

7. ¿Existen diferencias en términos de eficacia en la utilización de levonorgestrel frente al acetato de ulipristal como anticoncepción de urgencia?

8. ¿Existen diferencias en términos de eficacia en la utilización de la anticoncepción de urgencia hormonal frente a la intrauterina?

\subsection{Aspectos relacionados con la libre dispensación} de la anticoncepción de urgencia

9. ¿La libre dispensación de la anticoncepción de urgencia de levonorgestrel tiene un impacto sobre la incidencia de infecciones de transmisión sexual, las conductas sexuales de riesgo o el uso incorrecto de la anticoncepción habitual? 


\section{Manejo clínico de los métodos anticonceptivos}

\subsection{Consideraciones previas}

10. ¿Cuáles son los requisitos previos para la prescripción de un anticonceptivo hormonal?

11. ¿Cuáles son los controles necesarios para el seguimiento de un anticonceptivo hormonal?

12. ¿Cuáles son los requisitos previos para la inserción de un dispositivo intrauterino?

13. ¿Cuáles son los controles necesarios para el seguimiento en usuarias de un dispositivo intrauterino?

3.2. Manejo de las alteraciones del patrón de sangrado en anticoncepción

14. ¿Cómo se manejan los sangrados derivados del uso de anticoncepción?

\subsection{Eventualidades en el uso de la anticoncepción}

15. ¿Cómo se manejan los olvidos de las píldoras combinadas y de solo gestágeno?

16. ¿Cuál debe ser el manejo de la anticoncepción hormonal en caso de interacción medicamentosa?

\subsection{Manejo clínico de la anticoncepción intrauterina}

17. ¿Se debe utilizar la profilaxis antibiótica en alguna circunstancia en la inserción de un DIU?

18. ¿Es útil el uso de misoprostol para facilitar la inserción de un DIU?

\section{Situaciones específicas}

\subsection{Usuarias adolescentes}

19. ¿A qué edad puede considerarse la utilización de la anticoncepción hormonal en mujeres adolescentes?

20. ¿Es adecuada la utilización de un DIU en las mujeres adolescentes?

\subsection{Mujeres con edad reproductiva avanzada}

21. ¿Hasta qué edad es necesaria la anticoncepción hormonal?

22. ¿Se debe cambiar el DIU de cobre o LNG insertado después de los cuarenta años? 


\subsection{Sobrepeso y obesidad}

23. ¿Deben realizarse consideraciones especiales en usuarias de anticonceptivos hormonales o intrauterinos con sobrepeso u obesidad?

\subsection{Puerperio}

24. ¿En qué momento se puede iniciar un tratamiento anticonceptivo hormonal o intrauterino tras el parto? 



\section{Clasificación de la calidad de la evidencia y graduación de la fuerza de las recomendaciones}

\begin{tabular}{|c|c|c|c|c|}
\hline \multicolumn{5}{|c|}{ Clasificación de la calidad de la evidencia en el sistema GRADE } \\
\hline $\begin{array}{l}\text { Diseño del } \\
\text { estudio }\end{array}$ & $\begin{array}{c}\text { Calidad } \\
\text { inicial de la } \\
\text { evidencia }\end{array}$ & $\begin{array}{l}\text { Disminuir la calidad } \\
\text { si }\end{array}$ & $\begin{array}{l}\text { Aumentar la calidad } \\
\text { si }\end{array}$ & $\begin{array}{c}\text { Calidad } \\
\text { de la evidencia } \\
\text { final }\end{array}$ \\
\hline \multirow{2}{*}{$\begin{array}{l}\text { Ensayo clínico } \\
\text { aleatorizado } \\
\text { (ECA) }\end{array}$} & \multirow{2}{*}{ Alta } & \multirow{4}{*}{$\begin{array}{l}\text { Limitación en el } \\
\text { diseño: Importante (-1) } \\
\text { Muy importante (-2) } \\
\text { Inconsistencia: } \\
\text { Importante (-1) } \\
\text { Muy importante (-2) } \\
\text { Evidencia directa: } \\
\text { Importante (-1) } \\
\text { Muy importante (-2) } \\
\text { Imprevisión: } \\
\text { Importante (-1) } \\
\text { Muy importante (-2) } \\
\text { Sesgo de } \\
\text { publicación: } \\
\text { Alta probabilidad de (1) }\end{array}$} & \multirow{4}{*}{ 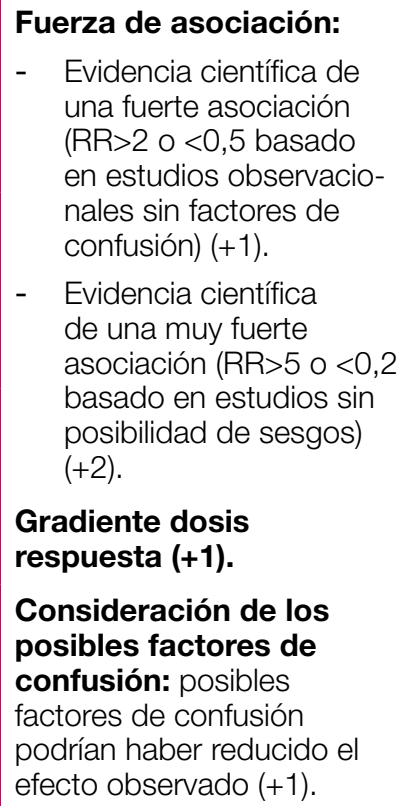 } & Alta \\
\hline & & & & Moderada \\
\hline \multirow{2}{*}{$\begin{array}{l}\text { Estudios } \\
\text { observacionales }\end{array}$} & \multirow[b]{2}{*}{ Baja } & & & Baja \\
\hline & & & & Muy baja \\
\hline
\end{tabular}

\begin{tabular}{|c|c|c|}
\hline \multicolumn{3}{|c|}{ Implicaciones de la fuerza de recomendación en el sistema GRADE } \\
\hline \multicolumn{3}{|c|}{ Implicaciones de una recomendación fuerte: } \\
\hline Usuarias & Clínicos & Gestores / Planificadores \\
\hline $\begin{array}{l}\text { La inmensa mayoría de } \\
\text { las personas estarían } \\
\text { de acuerdo con la } \\
\text { acción recomendada y } \\
\text { únicamente una pequeña } \\
\text { parte no lo estarían. }\end{array}$ & $\begin{array}{l}\text { La mayoría de usuarias deberían } \\
\text { recibir la intervención recomendada. }\end{array}$ & $\begin{array}{l}\text { La recomendación puede ser } \\
\text { adoptada como política sanitaria en } \\
\text { la mayoría de las situaciones. }\end{array}$ \\
\hline \multicolumn{3}{|c|}{ Implicaciones de una recomendación débil: } \\
\hline Usuarias & Clínicos & Gestores / Planificadores \\
\hline $\begin{array}{l}\text { La mayoría de las personas } \\
\text { estarían de acuerdo con la } \\
\text { acción recomendada pero } \\
\text { un número importante de } \\
\text { ellas no. }\end{array}$ & $\begin{array}{l}\text { Reconoce que diferentes opciones } \\
\text { serán apropiadas para diferentes } \\
\text { usuarias y que el profesional sanitario } \\
\text { tiene que ayudar a cada usuaria a } \\
\text { llegar a la decisión más consistente } \\
\text { con sus valores y preferencias. }\end{array}$ & $\begin{array}{l}\text { Existe necesidad de un debate } \\
\text { importante y la participación de los } \\
\text { grupos de interés. }\end{array}$ \\
\hline
\end{tabular}


En ocasiones el Grupo de Trabajo considera que existe algún aspecto práctico importante sobre el que se quiere hacer énfasis y para el cual no existe, probablemente, ninguna evidencia científica que lo soporte. En general estos casos están relacionados con algún aspecto del tratamiento considerado buena práctica clínica y que nadie cuestionaría habitualmente. Estos aspectos son valorados como puntos de buena práctica clínica. 


\section{Recomendaciones de la GPC}

\section{Eficacia y seguridad de los métodos anticonceptivos}

\begin{tabular}{|l|l|}
\hline Fuerte & $\begin{array}{l}\text { Se recomienda el uso de anticonceptivos hormonales e intrauterinos, debido } \\
\text { a que han mostrado una elevada efectividad. En la elección de un anticon- } \\
\text { ceptivo cada profesional sanitario debe considerar las circunstancias y pre- } \\
\text { ferencias de cada mujer. }\end{array}$ \\
\hline Fuerte & $\begin{array}{l}\text { Se recomienda que en la elección del anticonceptivo hormonal combinado } \\
\text { más adecuado cada profesional sanitario valore detalladamente los factores } \\
\text { de riesgo de tromboembolismo de la usuaria antes de iniciar el tratamiento } \\
\text { y periódicamente durante su uso. }\end{array}$ \\
\hline $\begin{array}{l}\text { Buente } \\
\text { práctica } \\
\text { clínica }\end{array}$ & $\begin{array}{l}\text { Se recomienda que cada profesional sanitario informe a las usuarias sobre el } \\
\text { beneficio derivado del uso de los anticonceptivos hormonales combinados y } \\
\text { del riesgo en términos absolutos de tromboembolismo, así como de los sig- } \\
\text { nos y síntomas de alerta que permitan su diagnóstico precoz. }\end{array}$ \\
\hline $\begin{array}{l}\text { Se sugiere la elaboración de protocolos para la provisión y manejo de an- } \\
\text { ticonceptivos en el marco del Sistema Nacional de Salud para asegurar la } \\
\text { ticonceptivos. }\end{array}$ \\
\hline
\end{tabular}

\section{Anticoncepción de urgencia}

\begin{tabular}{|l|l|}
\hline $\begin{array}{l}\text { Buena } \\
\text { práctica } \\
\text { clínica }\end{array}$ & $\begin{array}{l}\text { Es necesario informar a las mujeres que realizan una consulta de anticon- } \\
\text { cepción de urgencia sobre las opciones de anticoncepción de urgencia dis- } \\
\text { ponibles, de la mejor efectividad del DIU de cobre sobre los métodos orales, } \\
\text { y de la limitación en la efectividad dependiendo del tiempo transcurrido } \\
\text { desde la relación coital sin protección. }\end{array}$ \\
\hline Fuerte & $\begin{array}{l}\text { En aquellas situaciones en las que se decida insertar un DIU de cobre como } \\
\text { anticoncepción de urgencia, debe insertarse en las 120 horas (5 días) de ha- } \\
\text { ber mantenido relaciones coitales sin protección o haberse producido un } \\
\text { fallo del anticonceptivo utilizado. En el caso que pueda estimarse la fecha de } \\
\text { ovulación el dispositivo puede insertarse más tarde, siempre que no hayan } \\
\text { transcurrido 5 días tras esta fecha. }\end{array}$ \\
\hline Fuerte & $\begin{array}{l}\text { En aquellas situaciones en las que se decida utilizar un método oral como an- } \\
\text { ticoncepción de urgencia (acetato de ulipistral 30mg o levonorgestrel 1,5mg), } \\
\text { se recomienda administrar el tratamiento lo antes posible después de haber } \\
\text { mantenido relaciones coitales sin protección o haberse producido un fallo del } \\
\text { anticonceptivo utilizado (antes de las } 120 \text { horas (5 días) en el caso del acetato } \\
\text { de ulipristal o de las 72 horas (3 días) en el caso del levonorgestrel). }\end{array}$ \\
\hline
\end{tabular}




\begin{tabular}{|l|l|}
\hline Fuerte & $\begin{array}{l}\text { En aquellas situaciones en las que se decida utilizar un método oral como } \\
\text { anticoncepción de urgencia, se recomienda administrar de nuevo el anticon- } \\
\text { ceptivo si se producen vómitos en las tres horas siguientes a la toma del } \\
\text { comprimido. }\end{array}$ \\
\hline $\begin{array}{l}\text { Buena } \\
\text { práctica } \\
\text { clínica }\end{array}$ & $\begin{array}{l}\text { Se sugiere la elaboración de protocolos para la provisión y manejo de la } \\
\text { anticoncepción de urgencia que garantice la accesibilidad a las mujeres que } \\
\text { la necesiten. }\end{array}$ \\
\hline
\end{tabular}

\title{
Manejo clínico de los métodos anticonceptivos
}

\author{
Requisitos previos para la prescripción de un \\ anticonceptivo hormonal o dispositivo intrauterino
}

\begin{tabular}{|l|l|}
\hline $\begin{array}{l}\text { Buena } \\
\text { práctica } \\
\text { clínica }\end{array}$ & $\begin{array}{l}\text { Antes de prescribir un determinado anticonceptivo se sugiere realizar una } \\
\text { historia clínica que incluya una anamnesis detallada para identificar a mu- } \\
\text { jeres con factores de riesgo y antecedentes patológicos que contraindiquen } \\
\text { su uso, de acuerdo con los diferentes criterios de elegibilidad disponibles } \\
\text { (consultar Algoritmo 1). }\end{array}$ \\
\hline Fuerte & $\begin{array}{l}\text { Antes de prescribir un anticonceptivo hormonal combinado o un inyectable } \\
\text { de solo gestágenos se recomienda tomar la presión arterial y calcular el índi- } \\
\text { ce de masa corporal (consultar Algoritmo 1). }\end{array}$ \\
\hline Débil & $\begin{array}{l}\text { Se sugiere no realizar de manera rutinaria una exploración ginecológica ni } \\
\text { mamaria, o una citología, antes de prescribir un anticonceptivo (consultar } \\
\text { Algoritmo 1). }\end{array}$ \\
\hline
\end{tabular}

\section{Controles necesarios para el seguimiento de usuarias de anticonceptivos hormonales e intrauterinos}

\begin{tabular}{|l|l|}
\hline $\begin{array}{l}\text { Buena } \\
\text { práctica } \\
\text { clínica }\end{array}$ & $\begin{array}{l}\text { En el momento en que la mujer escoja un determinado método anticoncep- } \\
\text { tivo, se sugiere informarla sobre la conveniencia de consultar cualquier efec- } \\
\text { to secundario u otros problemas derivados del método anticonceptivo o si } \\
\text { desean cambiar el método. Las usuarias también deben recibir información } \\
\text { sobre los principales síntomas de alerta que permitan identificar situaciones } \\
\text { en las que deba considerarse una modificación en el uso del anticonceptivo. }\end{array}$ \\
\hline $\begin{array}{l}\text { Buena } \\
\text { práctica } \\
\text { clínica }\end{array}$ & $\begin{array}{l}\text { Se sugiere una visita a los tres meses tras el inicio de un tratamiento hormo- } \\
\text { nal combinado (oral, anillo o parche) o de sólo gestágeno, o a las 3-6 semanas } \\
\text { de la inserción en el caso del DIU. }\end{array}$ \\
\hline $\begin{array}{l}\text { Buena } \\
\text { práctica } \\
\text { clínica }\end{array}$ & $\begin{array}{l}\text { En las visitas de control, se sugiere comprobar cuál es su satisfacción con el } \\
\text { método y posibles problemas derivados de su uso. }\end{array}$ \\
\hline
\end{tabular}




\begin{tabular}{|l|l|}
\hline $\begin{array}{l}\text { Buena } \\
\text { práctica } \\
\text { clínica }\end{array}$ & $\begin{array}{l}\text { En las visitas de control, se sugiere explorar cualquier cambio en la historia } \\
\text { clínica de la usuaria que pueda causar un cambio en los criterios de elegibi- } \\
\text { lidad para el uso seguro de los anticonceptivos. }\end{array}$ \\
\hline Débil & $\begin{array}{l}\text { Se sugiere valorar cambios en el peso de la usuaria en cada visita de control } \\
\text { y discutir esta circunstancia con la usuaria que pueda asociar un eventual } \\
\text { cambio en el peso con el método anticonceptivo hormonal. }\end{array}$ \\
\hline Débil & $\begin{array}{l}\text { Se sugiere realizar una medición de la presión arterial en cada visita de con- } \\
\text { trol de las usuarias de anticonceptivos hormonales combinados (píldora, } \\
\text { parche o anillo). }\end{array}$ \\
\hline
\end{tabular}

\section{Manejo de las alteraciones del patrón de sangrado en anticoncepción}

\begin{tabular}{|l|l|}
\hline $\begin{array}{l}\text { Buena } \\
\text { práctica } \\
\text { clínica }\end{array}$ & $\begin{array}{l}\text { Antes de iniciar el tratamiento con un anticonceptivo hormonal o intrauteri- } \\
\text { no debe informarse a la usuaria de la modificación en su patrón de sangrado. } \\
\text { Principalmente en el caso de los dispositivos intrauterinos y los métodos } \\
\text { hormonales combinados la modificación del patrón del sangrado puede ser } \\
\text { habitual al inicio del uso del método, y tiende a desaparecer con su uso con- } \\
\text { tinuado. }\end{array}$ \\
\hline $\begin{array}{l}\text { Buena } \\
\text { práctica } \\
\text { clínica }\end{array}$ & $\begin{array}{l}\text { En el proceso de elección de un anticonceptivo hormonal o intrauterino } \\
\text { debe realizarse una anamnesis orientada a identificar factores de riesgo u } \\
\text { otras situaciones que permitan anticipar alteraciones en el patrón de san- } \\
\text { grado. }\end{array}$ \\
\hline Débil & $\begin{array}{l}\text { En usuarias de implante o inyectable se sugiere que ante la persistencia del } \\
\text { sangrado, en ausencia de problemas ginecológicos, se trate con antinflama- } \\
\text { torios no esteroideos. En caso de sangrado frecuente o prolongado se sugiere } \\
\text { un tratamiento con anticonceptivos combinados o estrógenos en dosis bajas. }\end{array}$ \\
\hline Débil & $\begin{array}{l}\text { En usuarias de anticonceptivos hormonales combinados se sugiere que ante } \\
\text { la persistencia del sangrado, y en ausencia de problemas ginecológicos, se } \\
\text { interrumpa el tratamiento durante } 3 \text { ó } 4 \text { días consecutivos. Estos días de } \\
\text { descanso del anticonceptivo, no están indicados en los primeros 21 días de } \\
\text { uso del método hormonal combinado en ciclos extendidos, ni está indicado } \\
\text { realizarlos en más de una ocasión en el mismo mes, ya que el anticonceptivo } \\
\text { puede reducir su eficacia. }\end{array}$ \\
\hline
\end{tabular}




\title{
Eventualidades en el uso de la anticoncepción
}

\author{
Manejo de los olvidos de las píldoras combinadas \\ y de solo gestágeno
}

\begin{tabular}{|l|l|}
\hline $\begin{array}{l}\text { Buena } \\
\text { práctica } \\
\text { clínica }\end{array}$ & $\begin{array}{l}\text { Durante una consulta de anticoncepción, cada profesional sanitario debería } \\
\text { informar sobre el posible impacto de un olvido y responder a las posibles } \\
\text { dudas de cada usuaria, valorando la posibilidad de proporcionar folletos in- } \\
\text { formativos sobre este aspecto. }\end{array}$ \\
\hline $\begin{array}{l}\text { Buena } \\
\text { práctica } \\
\text { clínica }\end{array}$ & $\begin{array}{l}\text { En caso de que se produzca un olvido en la toma de un anticonceptivo hor- } \\
\text { monal oral combinado, se sugiere considerar el momento en el que se produ- } \\
\text { jo el olvido y el número de píldoras olvidadas, para aconsejar a la usuaria la } \\
\text { manera más adecuada de retomar el uso adecuado del anticonceptivo (con- } \\
\text { sultar Algoritmo 3). }\end{array}$ \\
\hline $\begin{array}{l}\text { Buena } \\
\text { práctica } \\
\text { clínica }\end{array}$ & $\begin{array}{l}\text { En caso de que se produzca un olvido en la toma de un anticonceptivo hor- } \\
\text { monal de solo gestágeno, se sugiere considerar el momento en el que se pro- } \\
\text { dujo el olvido, para aconsejar a la usuaria la manera más adecuada de reto- } \\
\text { mar el uso adecuado del anticonceptivo (consultar Algoritmo 4). }\end{array}$ \\
\hline
\end{tabular}

\section{Anticoncepción hormonal e interacciones medicamentosas}

\begin{tabular}{|l|l|}
\hline $\begin{array}{l}\text { Buena } \\
\text { práctica } \\
\text { clínica }\end{array}$ & $\begin{array}{l}\text { Si una mujer desea iniciar un tratamiento con anticonceptivos hormonales, } \\
\text { se debe recopilar información sobre los tratamientos que está tomando o ha } \\
\text { tomado recientemente. }\end{array}$ \\
\hline $\begin{array}{l}\text { Buena } \\
\text { práctica } \\
\text { clínica }\end{array}$ & $\begin{array}{l}\text { Se debe advertir a la mujer que está utilizando un anticonceptivo hormo- } \\
\text { nal que ciertos medicamentos pueden alterar la eficacia del anticonceptivo } \\
\text { y que debe consultar a un médico antes de iniciar cualquier tratamiento me- } \\
\text { dicamentoso. }\end{array}$ \\
\hline $\begin{array}{l}\text { Buena } \\
\text { práctica } \\
\text { clínica }\end{array}$ & $\begin{array}{l}\text { Cuando se realice con la mujer la elección del anticonceptivo debe conside- } \\
\text { rarse la duración de un posible tratamiento concomitante, la causa por la que } \\
\text { se debe prescribir este tratamiento, y aquellas situaciones en las que puede } \\
\text { ser necesario tomar alguna medida contraceptiva adicional. }\end{array}$ \\
\hline $\begin{array}{l}\text { Buena } \\
\text { práctica } \\
\text { clínica }\end{array}$ & $\begin{array}{l}\text { En la elección de un anticonceptivo ante una posible interacción medica- } \\
\text { mentosa se sugiere consultar cualquiera de los documentos disponibles para } \\
\text { determinar los criterios médicos de elegibilidad para el uso de anticoncep- } \\
\text { tivos. }\end{array}$ \\
\hline
\end{tabular}

Manejo clínico de la anticoncepción intrauterina

\section{Profilaxis antibiótica en la inserción de un DIU}

\begin{tabular}{|l|l|}
\hline Fuerte & $\begin{array}{l}\text { No se recomienda administrar profilaxis antibiótica en la inserción de un } \\
\text { DIU. }\end{array}$ \\
\hline
\end{tabular}




\section{Uso de misoprostol durante la inserción de un DIU}

\begin{tabular}{|l|l|}
\hline Fuerte & $\begin{array}{l}\text { No se recomienda administrar misoprostol de manera rutinaria antes de la } \\
\text { inserción de un DIU con la finalidad de facilitar este procedimiento. }\end{array}$
\end{tabular}

\section{Situaciones específicas}

\section{Usuarias adolescentes}

\begin{tabular}{|l|l|}
\hline Débil & $\begin{array}{l}\text { Se sugiere ofrecer información a las mujeres adolescentes sobre los dife- } \\
\text { rentes tipos de anticonceptivos, destacando los beneficios adicionales de los } \\
\text { anticonceptivos reversibles de acción prolongada. }\end{array}$ \\
\hline $\begin{array}{l}\text { Buena } \\
\text { práctica } \\
\text { clínica }\end{array}$ & $\begin{array}{l}\text { Debe aconsejarse a las usuarias de DIU realizar una visita de seguimiento } \\
\text { en los primeros meses de uso para valorar su tolerancia, y recordarles que } \\
\text { deben consultar cualquier duda sobre el método anticonceptivo. }\end{array}$ \\
\hline
\end{tabular}

\section{Edad reproductiva avanzada}

\begin{tabular}{|l|l|}
\hline Fuerte & $\begin{array}{l}\text { Se recomienda no utilizar la edad de la usuaria como el único criterio en la } \\
\text { decisión sobre la interrupción de un anticonceptivo. }\end{array}$ \\
\hline $\begin{array}{l}\text { Buena } \\
\text { práctica } \\
\text { clínica }\end{array}$ & $\begin{array}{l}\text { Se sugiere que, en mujeres sin factores de riesgo ni problemas médicos rele- } \\
\text { vantes que desean evitar un embarazo no deseado, se mantenga la anticon- } \\
\text { cepción hormonal hasta la menopausia. }\end{array}$ \\
\hline $\begin{array}{l}\text { Buena } \\
\text { práctica } \\
\text { clínica }\end{array}$ & $\begin{array}{l}\text { Se sugiere que en la decisión de utilizar o continuar un determinado método } \\
\text { anticonceptivo en mujeres con edad reproductiva tardía, se individualice la } \\
\text { decisión teniendo en cuenta sus riesgos y beneficios, incluidos los no anti- } \\
\text { conceptivos. }\end{array}$ \\
\hline $\begin{array}{l}\text { Buena } \\
\text { práctica } \\
\text { clínica }\end{array}$ & $\begin{array}{l}\text { Se sugiere que las usuarias de un método anticonceptivo no hormonal como } \\
\text { el DIU de cobre interrumpan su uso transcurridos dos años tras el último } \\
\text { ciclo menstrual en las mujeres menores de 50 años, periodo que puede ser de } \\
\text { un año en las mujeres mayores de 50 años. }\end{array}$ \\
\hline $\begin{array}{l}\text { Buena } \\
\text { práctica } \\
\text { clínica }\end{array}$ & $\begin{array}{l}\text { Se sugiere informar a las usuarias de un DIU de cobre insertado a partir de } \\
\text { los 40 años sobre la posibilidad de mantener el dispositivo hasta la aparición } \\
\text { de la menopausia. }\end{array}$ \\
\hline $\begin{array}{l}\text { Buena } \\
\text { práctica } \\
\text { clínica }\end{array}$ & $\begin{array}{l}\text { Se sugiere extraer el DIU de levonorgestrel entre los } 40 \text { y } 44 \text { años a aquellas } \\
\text { mujeres a las que se les había insertado entre los } 35 \text { y } 39 \text { años. El DIU puede } \\
\text { renovarse o sustituirlo por un método alternativo. }\end{array}$ \\
\hline $\begin{array}{l}\text { Buena } \\
\text { práctica } \\
\text { clínica }\end{array}$ & $\begin{array}{l}\text { Se sugiere mantener el DIU de levonorgestrel durante siete años o hasta la } \\
\text { aparición de la menopausia en las usuarias en las que se les insertara el dis- } \\
\text { positivo a partir de los 45 años de edad. }\end{array}$ \\
\hline
\end{tabular}




\section{Sobrepeso y obesidad}

\begin{tabular}{|l|l|}
\hline $\begin{array}{l}\text { Buena } \\
\text { práctica } \\
\text { clínica }\end{array}$ & $\begin{array}{l}\text { En la elección de un método anticonceptivo para una mujer con sobrepeso } \\
\text { u obesidad se sugiere individualizar la decisión de acuerdo a las caracterís- } \\
\text { ticas de cada usuaria y a las directrices reflejadas en los criterios médicos de } \\
\text { elegibilidad para el uso de anticonceptivos, informando a la usuaria de las } \\
\text { ventajas e inconvenientes de cada uno de los métodos. }\end{array}$ \\
\hline Fuerte & $\begin{array}{l}\text { No se recomienda el uso de anticonceptivos hormonales combinados a las } \\
\text { mujeres con obesidad en las que se identifican factores de riesgo cardiovas- } \\
\text { cular adicional (edad avanzada, tabaquismo, diabetes, hipertensión), debido } \\
\text { a que aumenta el riesgo de sufrir alguna enfermedad cardiovascular secun- } \\
\text { daria. }\end{array}$ \\
\hline Débil & $\begin{array}{l}\text { En estas mujeres pueden considerarse métodos anticonceptivos que han } \\
\text { mostrado una buena eficacia sin aumentar el riesgo cardiovascular, como } \\
\text { los dispositivos intrauterinos o los métodos hormonales de sólo gestágeno. }\end{array}$ \\
\hline
\end{tabular}

\section{Puerperio}

\begin{tabular}{|l|l|}
\hline $\begin{array}{l}\text { Buena } \\
\text { práctica } \\
\text { clínica }\end{array}$ & $\begin{array}{l}\text { Se sugiere que los profesionales sanitarios promuevan durante el puerperio } \\
\text { espacios en los que se pueda tratar con las mujeres y sus parejas aspectos } \\
\text { relacionados con el consejo contraceptivo y la vivencia de la sexualidad en } \\
\text { esta etapa. }\end{array}$ \\
\hline $\begin{array}{l}\text { Buena } \\
\text { práctica } \\
\text { clínica }\end{array}$ & $\begin{array}{l}\text { Se sugiere consultar los criterios de elegibilidad disponibles para el uso de } \\
\text { anticonceptivos para individualizar la elección del método anticonceptivo } \\
\text { más adecuado de acuerdo con las características y antecedentes de cada } \\
\text { mujer. }\end{array}$ \\
\hline Fuerte & $\begin{array}{l}\text { Se recomienda informar sobre la necesidad de anticoncepción y la introduc- } \\
\text { ción de un método adaptado a la situación específica de las mujeres que de- } \\
\text { sean planificar sus futuros embarazos y que no mantienen lactancia materna } \\
\text { exclusiva. }\end{array}$ \\
\hline Débil & $\begin{array}{l}\text { Se sugiere informar sobre la posibilidad de seguir el método lactancia-ame- } \\
\text { norrea (MELA) hasta los 6 meses tras el parto a aquellas mujeres en las que } \\
\text { persiste la amenorrea, realizan lactancia materna exclusiva, y no presentan } \\
\text { riesgo de transmitir o adquirir una infección de transmisión sexual. }\end{array}$ \\
\hline
\end{tabular}




\section{Introducción}

La Estrategia Nacional de Salud Sexual y Reproductiva en el Sistema Nacional de Salud (SNS) reconoce los derechos sexuales y reproductivos como derechos humanos, tal y como se recoge en declaraciones, convenciones y pactos de las Naciones Unidas y otros documentos aprobados internacionalmente. El desarrollo de una sexualidad saludable pasa por el reconocimiento de los derechos sexuales, el derecho a la información y la decisión libre sobre la propia sexualidad del individuo (1).

En este sentido las personas deberían poder conocer las alternativas disponibles basadas en los resultados de la literatura científica disponible para que se puedan tomar decisiones bien informadas en situaciones como las de la elección de un método anticonceptivo.

La Encuesta Nacional de Salud Sexual de 2009 (2) muestra que el uso de métodos anticonceptivos para evitar embarazos no deseados es frecuente en el estado español. Hasta el $74 \%$ de las mujeres los usan siempre o casi siempre en la franja de edad entre los 16 a 44 años, en un porcentaje algo mayor que en los hombres (69\%) El método anticonceptivo más utilizados en parejas estables son, entre los hombres, el preservativo masculino (81\% entre los 16 y 24 años y el $51 \%$ entre los 25 a 54 años). En el caso de las mujeres, son varios los tipos de anticonceptivos utilizados. Las mujeres jóvenes usan en una mayor proporción el preservativo masculino (68\%), porcentaje que disminuye en las mujeres de 25 a 54 años (43\%). La píldora anticonceptiva se refiere como método anticonceptivo en el $33 \%$ de las mujeres entre 16 y 34 años, y disminuye al 18\% en las de 35 a 54 años. Aunque el uso de anticonceptivos como método de prevención de embarazos en parejas ocasionales muestra unas cifras similares, es importante destacar que hasta un $17 \%$ de los hombres y un $12 \%$ de las mujeres no usan ningún método, justificando la importancia de la realización de campañas informativas y educativas.

En junio-julio de 2018 se realiza la encuesta nacional sobre anticoncepción en España (Encuesta SEC 2018) $^{a}$, promovida por la Sociedad Española de Contracepción (SEC). En la misma se recoge que las mujeres que han mantenido relaciones sexuales alguna vez declaran haber utilizado algún método anticonceptivo la primera vez que tuvieron relaciones $(91,3 \%)$, sin embargo el $* 7,6 \%$ confiesa que la primera vez no utilizaron ningún método anticonceptivo.

Mientras que entre las mujeres de 45 a 49 años, el uso de método anticonceptivo en su primera relación fue en el $85,1 \%$ de los casos, esta práctica se incrementa en sucesivas generaciones (96,7\% entre las menores de 20 años). Reflejando una mayor sensibilidad en el uso de anticoncepción en su primera relación sexual entre las más jóvenes.

La encuesta actual, al igual que la publicada en 2009, describe que el preservativo es el método anticonceptivo utilizado actualmente por mayor número de mujeres en edad

a. Sociedad Española de Contracepción. Encuesta Nacional 2018 sobre la Anticoncepción en España. [Visitado: diciembre de 2018] Disponible en: http://sec.es/ 
fértil (29,6 \%), siendo su uso mayoritario en todas las franjas de edad. En segundo lugar se sitúa el uso de la píldora, que es referido por el 17,3\% de las mujeres.

La utilización de Dispositivos Intrauterinos se produce básicamente a partir de los 30 años, mientras que los métodos irreversibles femeninos se registran fundamentalmente a partir de los 35 años, cifras en consonancia con la encuesta publicada en 2009 (Encuesta SEC 2018).

Para facilitar que las personas tomen una decisión sobre el método anticonceptivo más adecuado a sus necesidades se requiere facilitar información accesible, completa y actualizada que les permita elegir entre las alternativas existentes. El acceso a las distintas opciones anticonceptivas, es un elemento clave para hacer efectivo el derecho de cada persona a tomar decisiones respecto a su vida reproductiva. Los equipos de profesionales sanitarios de centros de Atención Primaria o de Unidades de Atención a la Salud Sexual y Reproductiva deben disponer de información fiable y actualizada que les permita informar a las personas que desean utilizar un método anticonceptivo con la finalidad de promover las decisiones informadas y compartidas, evitando situaciones de inequidad.

Por este motivo, es imprescindible disponer de un documento con recomendaciones clínicas en las que se recojan las principales fuentes de incertidumbre, controversia, o variabilidad en la práctica en el manejo de situaciones específicas sobre anticoncepción. En esta Guía de Práctica Clínica se han identificado los aspectos más relevantes sobre la eficacia y seguridad de los anticonceptivos hormonales e intrauterinos, el manejo de la anticoncepción de urgencia, y el manejo clínico de estos métodos anticonceptivos prestando especial atención a poblaciones específicas, para ofrecer recomendaciones dirigidas a un uso informado de los anticonceptivos. 


\section{Alcance y objetivos}

\section{Objetivos}

Esta guía de práctica clínica (GPC) pretende servir como instrumento para disminuir la incertidumbre y la variabilidad en el manejo de los anticonceptivos hormonales e intrauterinos. Con esta GPC se busca:

1. Formular recomendaciones basadas en la literatura científica sobre el manejo clínico de la anticoncepción hormonal e intrauterina.

2. Ofrecer información actualizada y evaluada críticamente sobre una serie de aspectos determinantes en el proceso de decisión sobre la elección de un método anticonceptivo y su manejo clínico, que permita a los equipos de profesionales sanitarios mejorar la comunicación con las usuarias, ofreciéndoles información de calidad y accesible.

\section{Población diana}

La población diana de la guía son todas aquellas mujeres en edad fértil que deseen utilizar, o estén utilizando, un método anticonceptivo hormonal o intrauterino con la finalidad de evitar un embarazo no deseado. También se incluyen recomendaciones dirigidas a las mujeres que requieran utilizar un método de anticoncepción de urgencia.

\section{Áreas clínicas incluidas}

Esta Guía aborda una serie de aspectos de relevancia clínica relacionados con la utilidad de los anticonceptivos hormonales e intrauterinos y su manejo clínico en general o en situaciones específicas. De este modo la Guía recoge recomendaciones sobre los siguientes aspectos:

- Eficacia y seguridad de los diferentes anticonceptivos hormonales e intrauterinos.

- Uso de los anticonceptivos hormonales e intrauterinos en anticoncepción de urgencia.

- Manejo clínico de los anticonceptivos hormonales e intrauterinos relacionado con el inicio y control de su uso.

- Manejo de las alteraciones del patrón de sangrado en anticoncepción.

- Manejo clínico de situaciones específicas como el olvido de las píldoras, las interacciones medicamentosas de los anticonceptivos hormonales, la inserción de un DIU.

- Manejo clínico de los anticonceptivos hormonales e intrauterinos en usuarias en contextos concretos como la adolescencia, mujeres con una edad reproductiva avanzada, mujeres con sobrepeso u obesidad, o mujeres durante el puerperio. 
Esta Guía por el contrario no incluye aspectos relacionados con el resto de anticonceptivos, y en términos generales no trata sobre el uso de los anticonceptivos como método de prevención de infecciones de transmisión sexual. Por otro lado, la Guía no incluye recomendaciones relacionadas con la estructura asistencial o la organización de equipos para la prestación de servicios relacionados con la anticoncepción. En el estado español, aunque la prestación de servicios sobre anticoncepción está dirigida a la información, consejo y seguimiento de los métodos anticonceptivos, las Comunidades Autónomas muestran una estructura muy diversificada. La Estrategia de Salud Sexual y Reproductiva es un documento de referencia para obtener información relacionada con estos aspectos (1).

La Guía realiza de manera contínua referencias a los principales documentos disponibles de criterios elegibilidad para el uso de anticonceptivos (3-5). Estos documentos deben ser una referencia para consultar las indicaciones de los métodos anticonceptivos ante situaciones específicas, y permiten individualizar las decisiones ante situaciones concretas.

\section{Ámbito asistencial}

El área de aplicación de esta guía será el Sistema Nacional de Salud del estado español.

\section{Usuarios}

La población usuaria de esta guía son aquellos equipos de profesionales de la salud que prestan servicio en Centros de Atención Primaria, Unidades de Atención a la Salud Sexual y Reproductiva y Hospitales. 


\section{Metodología}

La metodología empleada para desarrollar esta Guía de Práctica Clínica ha seguido directrices del Manual Metodológico para la elaboración de Guías de Práctica Clínica del Sistema Nacional de Salud (6). Para la evaluación de la calidad de la evidencia y la graduación de la fuerza de las recomendaciones se han seguido las directrices metodológicas GRADE (7).

De forma general, las etapas realizadas han sido:

\section{Constitución del grupo de trabajo}

El grupo elaborador de la guía está formado por profesionales de atención primaria (medicina de familia y comunitaria, enfermería y matronas), y especializada (ginecología y obstetricia), con una amplia experiencia en el campo de la salud sexual y reproductiva. Además el grupo ha contado con especialistas en metodología pertenecientes al Centro Cochrane Iberoamericano.

\section{Formulación de las preguntas clínicas}

Las preguntas fueron estructuradas siguiendo un formato PICO (Población, Intervención, Comparación y Desenlaces [Outcomes]) y debatidas en el grupo elaborador durante una reunión de trabajo. En esta reunión se identificaron los diferentes apartados de la GPC y se realizó la priorización de las preguntas. La priorización se estableció teniendo en cuenta los objetivos, alcance, población diana, áreas clínicas y usuarios potenciales de la GPC.

Para evaluar los beneficios y la seguridad de las diferentes intervenciones incluidas, se priorizaron los desenlaces clave de interés para la población diana. El alcance de la Guía se focaliza en el uso de la anticoncepción como método para evitar embarazos no deseados, y por tanto de manera general en todas las preguntas clínicas se ha realizado un balance entre desenlaces clave relacionados con la tasa de embarazos como el principal desenlace de eficacia y desenlaces relacionados con la seguridad de los métodos. En preguntas clínicas más específicas se han identificado como clave desenlaces más específicos (como por ejemplo, la continuidad con el método en las preguntas sobre la anticoncepción en mujeres adolescentes, $u$ olvidos en preguntas relacionadas con la adherencia al tratamiento). La importancia de los diferentes desenlaces valorados puede consultarse en el Apéndice 1 que recoge las tablas GRADE de evaluación de la calidad de la evidencia y resumen de los resultados.

\section{Búsqueda bibliográfica}

En una primera etapa se identificaron GPC y otros documentos con recomendaciones sobre el uso de anticonceptivos. Esta etapa llevó a identificar documentos relacionados con los criterios de elegibilidad para el uso de anticonceptivos $(4,5)$ y otros documentos relevantes producidos por sociedades científicas $(3,8)$. 
Posteriormente, se realizó una búsqueda global de revisiones sistemáticas (RS) y otros documentos de síntesis crítica de literatura científica, etapa en la que se consultaron las siguientes bases de datos electrónicas:

- $\quad$ NHS Evidence

- Cochrane Database of Systematic Reviews (The Cochrane Library)

- Database of Abstracts of Reviews of Effects (DARE)

- Health Technology Assessment (HTA) Database

- $\quad$ NHS Economic Evaluation Database (NHS EED)

- MEDLINE (accedido mediante PubMed)

Finalmente, se realizaron búsquedas específicas de estudios individuales para actualizar las revisiones sistemáticas relevantes y para las preguntas en las cuales no se identificaron estudios en la primera fase. Se buscaron específicamente ensayos clínicos aleatorizados (ECA) y estudios observacionales, a partir de la fecha de búsqueda de las revisiones sistemáticas relevantes. Estas búsquedas se ejecutaron en MEDLINE y The Cochrane Central Register of Controlled Trials. Para identificar estudios que apoyaran la justificación para la formulación de las recomendaciones se consultaron la base de datos NHS Economic Evaluation Database para evaluaciones económicas, y MEDLINE para identificar estudios en el apartado de valores y preferencias.

No se estableció ningún límite lingüístico ni temporal a las búsquedas llevadas a cabo. Se han realizado búsquedas de manera rutinaria hasta el mes de agosto de 2016. Las principales estrategias de búsqueda diseñadas originalmente se muestran en el Apéndice 2.

\section{Selección de los estudios y evaluación de la calidad de la evidencia}

Para la selección de los estudios, se realizó un cribado inicial por título y resumen para determinar su pertinencia respecto a las preguntas a responder en la GPC. Las referencias consideradas pertinentes fueron evaluadas a texto completo para determinar su calidad e inclusión en la GPC. Se eligieron aquellas referencias con un menor riesgo de sesgo, que aportaran datos sobre los desenlaces de interés elegidos, priorizándose las revisiones sistemáticas y posteriormente, los ensayos clínicos aleatorizados u otros estudios observacionales en el caso de que fuera necesario.

La evaluación de la calidad de la evidencia se realizó siguiendo las directrices del grupo internacional de trabajo GRADE (Grading of Recommendations of Assessment Development and Evaluations) (7, 9). Para cada desenlace identificado como clave o importante para cada pregunta clínica, se evaluó la calidad del cuerpo de la evidencia disponible. Se consideraron los siguientes factores que pueden modificar la confianza en los resultados disponibles en la literatura científica: riesgo de sesgo, consistencia entre los resultados de diferentes estudios, la disponibilidad de evidencia directa, previsión de los estimadores del efecto y el sesgo de publicación. Ante la presencia de estos factores la calidad de la evidencia disminuye dependiendo la magnitud de su impacto sobre la confianza en los resultados de la literatura. Además también se valoran los siguientes factores que 
podrían aumentar la calidad de la evidencia, caso que aplica generalmente a los estudios observacionales: tamaño del efecto, relación dosis-respuesta, impacto en los resultados de los factores de confusión. Todo el proceso de evaluación permitió clasificar la calidad de la evidencia para cada desenlace en cuatro categorías: alta, moderada, baja, o muy baja. Ante la disponibilidad de ensayos clínicos se considera que la calidad de la evidencia es alta, que puede disminuir ante la presencia de los factores mencionados anteriormente. Cuando se dispone de estudios observacionales, la calidad de la evidencia inicial es baja y, en ausencia de limitaciones relevantes, se puede considerar aumentar la calidad de la evidencia, de acuerdo a los factores que se han comentado. Finalmente se realiza una clasificación global de la calidad de la evidencia para cada pregunta clínica, que implica realizar un juicio general de la calidad entre los desenlaces clave para una determinada pregunta clínica. Las valoraciones relativas a la calidad de la evidencia, en forma de tablas de resumen de resultados, se detallan en el Apéndice 1. Para las diferentes preguntas clínicas se recoge el análisis de la calidad de la evidencia por cada desenlace de interés, conjuntamente con los estimadores del efecto obtenidos de los estudios evaluados. La justificación de las consideraciones realizadas para clasificar la calidad de la evidencia y la extracción de datos de los estudios se detallan con explicaciones en forma de notas a pie de tabla.

\section{Elaboración de las recomendaciones}

A partir del resumen de la evidencia disponible para cada pregunta clínica, se pasó a la formulación de recomendaciones siguiendo la metodología GRADE. Para determinar la dirección (a favor o en contra de una intervención) y la fuerza de las recomendaciones (fuertes o débiles), se ponderaron los resultados de la calidad global de la evidencia disponible, el balance entre los beneficios y los riesgos de cada uno de los procedimientos evaluados; los aspectos relacionados con los valores y preferencias de las usuarias y según la pregunta evaluada, el uso de recursos y costes asociados.

Las consideraciones necesarias para la formulación de las recomendaciones se recogen en un apartado denominado "De la evidencia a la recomendación". En este apartado se recoge:

- una breve síntesis de la evidencia valorada en cada apartado acompañado de la clasificación de su calidad (bajo la sección "Resumen de la evidencia");

- la discusión sobre la calidad de la evidencia global de cada pregunta clínica con los principales aspectos que afectan a la confianza en la literatura evaluada (bajo la sección "Calidad de la evidencia");

- discusión sobre aspectos que determinan cómo las usuarias valoran la importancia de los diferentes desenlaces relevantes para tomar decisiones (bajo la sección "Valores y preferencias de las usuarias");

- discusión sobre el balance entre efectos deseables e indeseados de los diferentes aspectos valorados en las preguntas clínicas (bajo las secciones "Beneficios y riesgos/carga de las intervenciones" y "Balance beneficio/riesgo");

- valoración del uso de recursos y costes de los procedimientos valorados en las preguntas clínicas (bajo la sección "Uso de recursos y costes"); y

- finalmente se recoge una justificación de las recomendaciones formuladas, en la que se discuten cuáles son los principales aspectos que determinan tanto la direc- 
ción como la fuerza de éstas. Como se ha comentado, la fuerza de las recomendaciones se gradúa como fuerte o débil, dependiendo de la confianza que se puede tener en el hecho de que implantar una determinada recomendación comporte más beneficios que inconvenientes en las usuarias. En ocasiones el Grupo de Trabajo considera que existe algún aspecto práctico importante sobre el que se quiere hacer énfasis y para el cual no existe, probablemente, ninguna evidencia científica que lo soporte. En general estos casos están relacionados con algún aspecto del tratamiento considerado buena práctica clínica y que nadie cuestionaría habitualmente. Estos aspectos son valorados como puntos de buena práctica clínica.

Las recomendaciones se incluyen al final de cada pregunta clínica y al principio de este documento, en el apartado "Recomendaciones de la GPC".

\section{Revisión externa}

Con el objeto de mejorar la calidad, evaluar la pertinencia y aplicabilidad de las recomendaciones, así como la claridad, congruencia e información subyacente, un grupo de personas revisó el borrador del documento. Todos los cometarios fueron registrados y tabulados para dar respuesta a las personas revisoras y aquellos comentarios clave que afectaron a la pertinencia o sentido de una recomendación fueron discutidos posteriormente con el grupo de trabajo.

\section{Conflictos de intereses}

La declaración del conflicto de intereses, fue elaborada a través de un proceso que se inició con el envio de un formulario a cada uno de los autores y revisores externos que formaron parte del grupo de trabajo de esta GPC. Se recogieron (Anexo 4) los conflictos que sólo hayan estado relacionados con el alcance de la GPC.

\section{Actualización}

Está prevista una actualización de la guía cada tres años, o en un plazo de tiempo inferior si aparece nueva literatura científica que pueda modificar algunas de las recomendaciones ofrecidas en esta guía. Las actualizaciones se realizarán sobre la versión electrónica de la guía, disponible en www.guiasalud.es y siguiendo las recomendaciones del Manual Metodológico para la actualización de Guías de Práctica Clínica del Sistema Nacional de Salud vigente (6). 


\section{Eficacia y seguridad de los métodos anticonceptivos}

Preguntas a responder:

- ¿Cuál es la eficacia y efectividad de los anticonceptivos hormonales?

- ¿Existe alguna diferencia entre los intervalos libres de hormona en la anticoncepción hormonal combinada (24/4 vs 21/7), en términos de eficacia y efectos adversos?

- ¿Cuál es la eficacia y efectividad de los anticonceptivos intrauterinos?

- ¿En qué casos deben realizarse consideraciones especiales en usuarias de anticonceptivos hormonales en relación con el riesgo de tromboembolismo venoso?

- ¿En qué casos deben realizarse consideraciones especiales en usuarias de anticonceptivos hormonales en relación con el riesgo de tromboembolismo arterial?

\subsection{Anticonceptivos hormonales e intrauterinos}

\subsubsection{Eficacia y efectividad de los anticonceptivos hormonales e intrauterinos}

Se han identificado datos sobre la eficacia de los métodos anticonceptivos hormonales en una revisión sistemática de la literatura (10) y dos revisiones narrativas sobre la contracepción hormonal (11) y sobre los fallos de los métodos anticonceptivos en EEUU (12). Adicionalmente se comentan los resultados de cuatro estudios observacionales que han aportado datos sobre la eficacia de los métodos anticonceptivos orales en EEUU $(13,14)$ y Europa $(15,16)$, que no habían sido considerados en las revisiones de la literatura mencionadas. También se han recogido los resultados del estudio CHOICE, cohorte prospectiva que evalúa un programa comunitario dirigido a promover el uso de anticonceptivos reversibles de acción prolongada (LARC por sus siglas en inglés) (17).

Los estudios evaluados expresan la eficacia de los métodos anticonceptivos con el índice de Pearl (número de embarazos no deseados por cada 1200 ciclos de uso (en 100 mujeres/año)), a partir de tablas de vida. La tabla muestra la eficacia de varios métodos anticonceptivos en términos de su uso ideal (eficacia del método) y su uso habitual (efectividad del método) (11). Para tener una referencia que permita comparar la eficacia de los diferentes métodos, la tabla incluye tanto los anticonceptivos hormonales, como los intrauterinos, como el resto de métodos.

Con un uso ideal, el fallo de los métodos anticonceptivos hormonales oscila entre los 0,05 embarazos no deseados del implante subcutáneo de gestágeno a los 0,3 de los anticon- 
ceptivos orales combinados. No obstante, hasta la mitad de las usuarias de anticonceptivos tienen un olvido en cada ciclo, lo que puede aumentar el fallo de los métodos anticonceptivos considerablemente (reflejado en el índice de Pearl referido al uso habitual). En el caso de los anticonceptivos orales, el riesgo de embarazo es mayor cuando el olvido se produce al principio de la primera semana del ciclo, o al final de la última semana.

TABLA. Incidencia de embarazos no deseados durante el primer año de uso por cada 100 mujeres (fallo del método por cada 100 mujeres/año; índice de Pearl: uso habitual (efectividad) y uso ideal (eficacia)).

[adaptada de Trussell 2004 (18), Wiegratz 2011 (11)

(ajustes a los resultados de Mansour 2010 (10), Dinger 2011(14)) y Trussell 2011 (12)]

\begin{tabular}{|c|c|c|c|c|}
\hline \multirow[t]{2}{*}{ Método } & \multicolumn{2}{|c|}{ Uso ideal } & \multicolumn{2}{|c|}{ Uso habitual } \\
\hline & $\begin{array}{c}\text { Trussell } 2004 \\
\text { (18) } \\
\text { Wiegratz } 2011 \\
(11)\end{array}$ & $\begin{array}{c}\text { Trussell } 2011 \\
\text { (12) }\end{array}$ & $\begin{array}{l}\text { Trussell } 2004(18) \\
\text { Wiegratz } 2011(11)\end{array}$ & $\begin{array}{c}\text { Trussell } 2011 \\
\text { (12) }\end{array}$ \\
\hline Ningún método & \multicolumn{2}{|c|}{85} & \multicolumn{2}{|c|}{85} \\
\hline $\begin{array}{l}\text { Anticonceptivos orales } \\
\text { combinados }\end{array}$ & \multicolumn{2}{|c|}{0,3} & $8\left(2,2^{1,2}\right)$ & 9 \\
\hline Parche transdérmico & \multicolumn{2}{|c|}{0,3} & $8\left(1,2^{2}\right)$ & 9 \\
\hline Anillo vaginal & \multicolumn{2}{|c|}{0,3} & $8\left(1,2^{2}\right)$ & 9 \\
\hline Píldora de progestágeno solo & \multicolumn{2}{|c|}{0,3} & 8 & 9 \\
\hline $\begin{array}{l}\text { Píldora de progestágeno solo } \\
\text { (desogestrel) }\end{array}$ & $0,14^{1}$ & - & $0,41^{1}$ & - \\
\hline $\begin{array}{l}\text { Progestágenos inyectables } \\
\text { (acetato demedroxiprogesterona) }\end{array}$ & 0,3 & 0,2 & 3 & 6 \\
\hline Implante subcutáneo de gestágeno & \multicolumn{2}{|c|}{0,05} & \multicolumn{2}{|c|}{0,05} \\
\hline DIU de levonorgestrel & \multicolumn{2}{|c|}{0,2} & \multicolumn{2}{|c|}{0,2} \\
\hline Esterilización femenina & \multicolumn{2}{|c|}{0,5} & \multicolumn{2}{|c|}{0,5} \\
\hline Esterilización masculina & \multicolumn{2}{|c|}{0,10} & \multicolumn{2}{|c|}{0,15} \\
\hline DIU de cobre & \multicolumn{2}{|c|}{0,6} & \multicolumn{2}{|c|}{0,8} \\
\hline Diafragma con espermicida & \multicolumn{2}{|c|}{6} & 16 & 12 \\
\hline Preservativo masculino & \multicolumn{2}{|c|}{2} & 15 & 18 \\
\hline Preservativo femenino & \multicolumn{2}{|c|}{5} & \multicolumn{2}{|l|}{21} \\
\hline Espermicida & \multicolumn{2}{|c|}{18} & 29 & 28 \\
\hline Esponja vaginal en nulíparas & \multicolumn{2}{|c|}{9} & 16 & 12 \\
\hline Esponja vaginal en no nulíparas & \multicolumn{2}{|c|}{20} & 32 & 24 \\
\hline Coito interrumpido & \multicolumn{2}{|l|}{4} & 27 & 22 \\
\hline Métodos naturales & \multicolumn{2}{|l|}{$3-5$} & & 25 \\
\hline
\end{tabular}

Una revisión sistemática [(10), fecha de búsqueda 2008) ha recopilado las tasas de fallo de algunos métodos anticonceptivos a partir de la evaluación de 139 estudios (ensayos clínicos aleatorizados y estudios observacionales prospectivos]. De los estudios valorados, 
la mayoría evaluaban la eficacia de métodos anticonceptivos hormonales: 47 estudios evaluaron anticonceptivos orales combinados, uno evaluó la píldora de solo progestágeno, tres el parche transdérmico, tres el anillo vaginal, 15 estudios evaluaron implantes, 16 métodos inyectables, y nueve el DIU liberador de levonorgestrel $(20 \mu \mathrm{g} / \mathrm{d}$, por 5 años $)$. Por otro lado, 31 estudios presentaron resultados sobre el DIU de cobre.

La edad media de las mujeres varió dependiendo del método anticonceptivo utilizado: de 23 a 26 años en los anticonceptivos orales combinados, de 28 a 29 años en el parche, de 27 a 28 años en el anillo, entre los 25 y los 30 en los implantes, de 23 a 29 años en los inyectables, y de 25 a 35 años en los DIU de levonorgestrel.

En términos generales se utilizó el índice de Pearl para los métodos anticonceptivos de acción corta (anticonceptivos orales, píldora de solo pro-

Calidad moderada gestágeno, parche y anillo) mientras que para los anticonceptivos de acción prolongada se estimó su eficacia en tablas de vida. En este sentido, la revisión de Mansour 2010 describe que el índice de Pearl para los anticonceptivos de acción corta es de alrededor del 2,5 por cada 100 mujeres en el primer año de uso (tanto para el uso ideal como habitual) (10). Las tablas de vida para los anticonceptivos de acción prolongada (implantes y DIU de levonorgestrel) muestran un riesgo de fallo entre el 0,06 y 1,1 de embarazos no deseados al año. En el caso del DIU de cobre, la tasa anual de fallo está entre el 0,1 y el 1,4 por 100 mujeres/año. Los datos de eficacia para los métodos anticonceptivos hormonales descritos en la revisión de Mansour se pueden consultar en el Anexo 1. Se han seleccionado los estudios con población europea, teniendo en cuenta que, como se discute más abajo, los datos sobre el fallo de los métodos anticonceptivos son mayores en los estudios con mujeres norteamericanas que en los estudios con participantes europeas.

A la luz de los resultados de la revisión, los autores destacan la eficacia del DIU de levonorgestrel y los implantes, seguidos de los métodos anticonceptivos de acción corta. Respecto a los anticonceptivos orales combinados se destaca que a pesar que el índice de Pearl es ligeramente superior en el caso de los anticonceptivos con una dosis de etinilestradiol de $20 \mu \mathrm{g}(0 \mathrm{a}$ 1,6 en su uso habitual) que en los anticonceptivos con una dosis de $30 \mu \mathrm{g}$ ( 0 a 1,19 en su uso habitual), estos resultados se solapan de manera considerable, y por lo tanto en la práctica clínica habitual ambas formulaciones tendrían una efectividad similar, tal y como han comentado otros autores (11). En concreto, una revisión Cochrane ((19) fecha de búsqueda 2010) no encontró diferencias significativas en la eficacia contraceptiva de 13 formulaciones orales que contenían dosis de etinilestradiol de $20 \mu \mathrm{g}$ o superiores. La revisión destaca que, aunque los anticonceptivos con una dosis de $20 \mu \mathrm{g}$ parecen ser más seguros, los ensayos clínicos disponibles no tienen el poder suficiente para demostrar esta hipótesis. En el caso de las modificaciones de los patrones de sangrado asociados a las dosis bajas de estrógenos, los autores atribuyen este hecho al posible impacto de los diferentes tipos de gestágeno. 
En una revisión narrativa (12) se actualizan los datos de eficacia de los anticonceptivos disponibles en EEUU. Como se ha reflejado en la tabla de incidencia de embarazos no deseados durante el primer año de uso, los datos sobre el uso ideal se mantienen para todos los anticonceptivos hormonales, excepto para los progestágenos inyectables (el índice de Pearl pasa de 0,3 a 0,2 ). En cuanto a los datos de uso habitual el riesgo de fallo del método aumenta en el caso de los anticonceptivos orales, el parche, el anillo y la píldora de solo progestágeno (índice de Pearl 9 por 100 mujeres en el primer año de uso), con un aumento considerable en el caso de los progestágenos inyectables (índice de Pearl 6 por 100 mujeres en el primer año de uso). Estos datos muestran que en términos generales los datos de fallo del método son mayores en los estudios que recopilan datos de mujeres norteamericanas que de mujeres europeas.

En este sentido, una serie de estudios permiten comparar las tasas de fallo del método entre mujeres estadounidenses y europeas. Un estudio transversal estimó la eficacia de los anticonceptivos más utilizados en EEUU (en términos de fallo del método) a partir de la encuesta de salud National Survey of Family Growth (13). El estudio mostró una tasa global de fallo del método anticonceptivo del 12,4 por cada 100 mujeres durante el primer año de uso, siendo los métodos inyectables los métodos más eficaces (fallo del método en su uso habitual de 6,7 (IC95\% 4,3 a 10,4) por cada $100 \mathrm{mu}$ jeres durante el primer año de uso). No se observaron mejoras en la tasa de fallo del método cuando se compararon los resultados de la encuesta de salud del año 2002 y del año 1995, con un fuerte impacto de las variables socioeconómicas sobre el riesgo de embarazo no deseado.

Por otro lado, un estudio ha presentado los resultados de las participantes estadounidenses en la cohorte prospectiva controlada International Active Surveillance of Women Taking Oral Contraceptives (14), con datos de 73.269 mujeres/año. El estudio se centró en estimar los datos sobre la eficacia de los anticonceptivos orales (en términos de fallo del método en su uso habitual), disponibles en EEUU. El estudio incluyó a mujeres que usaban un anticonceptivo oral por primera vez (el $80 \%$ de mujeres eran usuarias que habían cambiado de método), con una edad media de 26,3 años y un índice de masa corporal (IMC) de $26,3 \mathrm{~kg} / \mathrm{m}^{2}$. El estudio mostró una incidencia de 1634 embarazos no deseados, la mayoría de ellos relacionados con un olvido (46,3\%). El índice de Pearl referido al uso habitual en el estudio fue de 2,2 (IC95\% 2,1 a 2,3). Los anticonceptivos combinados de drospirenona y etinilestradiol (EE) administrados en un régimen de 24 días mostraron un índice de Pearl menor (1,6; IC95\% 1,4 a 1,9) que los anticonceptivos administrados en un régimen de 21 días (2,2; IC95\% 1,8 a 2,6), o el resto de anticonceptivos orales evaluados (combinación de EE y acetato de noretisterona administrado en regimenes de 21 o 24 días; 2,6; IC95\% 2,4 a 2,7). 
El riesgo de fallo del método fue significativamente mayor en el régimen de 21 días y el resto de anticonceptivos orales comparado con el régimen de 24 días (HR 0,7; IC95\% 0,6 a 0,8). La edad fue el mayor predictor de fallo del método, ya que las mujeres entre las edades de 20 y 24 años mostraron un índice de Pearl 3 veces superior ( 3 fallos por 100 mujeres/año) que las mujeres de 35 a 39 años. Un cambio en el IMC de los 20 a $25 \mathrm{~kg} / \mathrm{m} 2$ a los 30 a 35 $\mathrm{kg} / \mathrm{m} 2$ solamente repercutió en un incremento del índice de Pearl de los 2 a los 2,5 fallos por 100 mujeres/año. Los datos de las tablas de vida muestran como el riesgo de fallo del método aumentaron con el tiempo de uso:

\begin{tabular}{|l|c|c|c|c|}
\hline \multirow{2}{*}{ Método } & \multirow{2}{*}{ Participantes } & \multicolumn{3}{|c|}{ Fallo del método (\%) al final del año (IC95\%) } \\
\cline { 3 - 5 } & $\mathbf{1}$ & $\mathbf{2}$ & $\mathbf{3}$ \\
\hline $\begin{array}{l}\text { Drospirenona/ } \\
\text { EE 24 días }\end{array}$ & 10.302 & $2,1(1,7$ a 2,4$)$ & $3,4(2,9$ a 4,0$)$ & $4,7(3,8$ a 5,6$)$ \\
\hline $\begin{array}{l}\text { Drospirenona/ } \\
\text { EE 21 días }\end{array}$ & 3.982 & $2,8(2,2$ a 3,3) & $4,5(3,6$ a 5,4$)$ & $5,7(4,5$ a 6,9$)$ \\
\hline Otros métodos & 12.875 & $3,5(3,3$ a 3,7) & $5,4(5,1$ a 5,7$)$ & $6,7(6,2$ a 7,1$)$ \\
\hline
\end{tabular}

Los resultados de estos dos estudios se contraponen con los resultados del estudio EURAS, una cohorte prospectiva controlada con datos de 112.659 mujeres/año europeas para evaluar la seguridad de una combinación de drospirenona 3mg con EE 30 $\mathrm{gg}(15,16)$. La edad media de las participantes en este estudio fue de 25,2 $\pm 8,2$ años, con un peso medio de $63,1 \pm 12,1 \mathrm{~kg}, \mathrm{y}$ un IMC $22,1 \pm 4,1 \mathrm{~kg} / \mathrm{m}^{2}$. El 20\% de las participantes usaron anticonceptivos orales por primera vez. Los anticonceptivos evaluados en el estudio fueron drospirenona, levonorgestrel, acetato de clormadinona (ACM), desogestrel, y dienogest.

En esta cohorte de mujeres se recogieron 545 embarazos no deseados lo que supone un índice de Pearl global referido al uso habitual del 0,48 fallos por 100/mujeres año (IC95\% 0,44 a 0,53), la mayoría atribuibles a un olvido $(42,2 \%)$. Los anticonceptivos mostraron los siguientes datos de efectividad desagregados:

\begin{tabular}{|l|c|c|}
\hline \multicolumn{1}{|c|}{ Método } & Indice de Pearl & $\begin{array}{c}\text { Tablas de vida: fallo del método (\%) } \\
\text { al final del primer año de uso (IC95\%) }\end{array}$ \\
\hline Anticonceptivos orales & $0,48(0,44$ a 0,53$)$ & $0,75(0,68$ a 0,82$)$ \\
\hline ACM & $0,48(0,35$ a 0,63$)$ & $0,78(0,57$ a 1,06$)$ \\
\hline Desogestrel & $0,50(0,40$ a 0,63$)$ & $0,88(0,69$ a 1,12$)$ \\
\hline Dienogest & $0,40(0,30$ a 0,53$)$ & $0,57(0,41$ a 0,79$)$ \\
\hline Drospirenona & $0,44(0,37$ a 0,53$)$ & $0,62(0,50$ a 0,76$)$ \\
\hline Levonorgestrel & $0,53(0,46$ a 0,62$)$ & $0,78(0,57$ a 1,06$)$ \\
\hline
\end{tabular}


No se observaron diferencias significativas entre el riesgo de embarazo no deseado entre los diferentes tipos de anticonceptivos evaluados. La eficacia de estos métodos anticonceptivos no varió en función del IMC de las mujeres (HR 1,00; IC95\% 0,98 a 1,03), excepto en el caso del acetato de clormadinona en el que se observó un aumento del riesgo de embarazo entre las mujeres con un IMC superior a los $30 \mathrm{~kg} / \mathrm{m}^{2}$. Estos resultados también se observaron al analizar la eficacia de los anticonceptivos dependiendo del peso de las mujeres. Como en otros estudios, se observó un claro declive del riesgo de embarazo no deseado a partir de los 30 años. Las diferencias en la eficacia de los anticonceptivos con una dosis de EE superior o inferior a los $30 \mu \mathrm{g}$ no fueron clínicamente relevantes (índice de Pearl EE $<30 \mu \mathrm{g}$ 0,57 (IC95\% 0,46 a 0,69) frente a $\geq 30 \mu \mathrm{g} 0,47$ (IC95\% 0,42 a 0,52), resultado similar al observado al comparar los régimenes multifásicos con los monofásicos (índice de Pearl multifásico 0,51 (IC95\% 0,39 a 0,65) frente a monofásico 0,48 (IC95\% 0,44 a 0,53).

El estudio CHOICE mostró una mayor tasa de fallo de la píldora, el parche o el anillo (PPA) que los anticonceptivos LARC. El porcentaje de fallos a 1, 2 y 3 años para el PPA fue de 4,8\%,7,8\% y 9,4\% respectivamente, que fue significativamente mayor que la de los DIU o implantes $(0,3 \%, 0,6 \%, 0,9 \%$; $\mathrm{P}<0,001$ ), o el inyectable de acetato de medroxiprogesterona (DMPA). Los datos sobre la incidencia acumulada mostraron un mayor riesgo de fallo de los métodos PPA (4,55 fallos por 100 usuarias/año) frente al inyectable de DMPA (0,22 por 100 usuarias/año) o los DIU o implante $(0,27$ por 100 usuarias/año) (HR: 21,8; IC95\% 13,7 a 34,9) (17).

\subsubsection{Intervalos libres de hormona en la anticoncepción oral combinada}

Se han identificado seis ensayos clínicos que comparan el uso de anticonceptivos hormonales orales con un intervalo libre de hormona de siete (21/7) o cuatro días (24/4) (20-25) y un estudio de cohorte prospectivo centrado en la seguridad de diferentes regímenes de anticonceptivos, entre ellos, el de 24/4 (26). Solamente se han recogido los resultados de los ensayos clínicos que mostraron datos sobre los embarazos y cumplimiento. Las diferencias entre los estudios en la descripción de los desenlaces de interés no hicieron posible combinar sus resultados en un metanálisis. Todos ellos fueron estudios abiertos y con financiación privada.

Un ensayo clínico aleatorizó a 938 mujeres a recibir durante seis ciclos un régimen de 24/4 de acetato de noretisterona (NETA $1 \mathrm{mg} / \mathrm{EE} 20 \mu$ ) o un régimen de 21/7 del mismo anticonceptivo (24). Otro ensayo aleatorizó a 1074 mujeres a recibir durante seis ciclos un régimen de 24/4 gestodeno (GSD $60 \mu / \mathrm{EE} 15 \mu$ ) o un régimen de 21/7 de desogestrel (DSG $150 \mu / \mathrm{EE} 20 \mu)(21)$.

Dos ensayos clínicos han evaluado la eficacia de un régimen de 24/4 de drospirenona (DRSP $3 \mathrm{mg} / \mathrm{EE} 20 \mu$ ). En el primero se aleatorizaron a 453 mujeres a recibir siete ciclos 


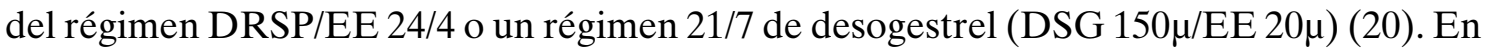
el otro se aleatorizaron a 355 a recibir solamente 3 ciclos de DRSP/EE 24/4 o un régimen

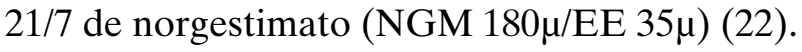

Finalmente, dos ensayos clínicos más han evaluado la eficacia de un régimen 24/4 de acetato de nomegestrol (NOMAC 2,5 mg/EE 1,5 mg) frente a un régimen 21/7 de drospirenona (DRSP $3 \mathrm{mg} / \mathrm{EE} 20 \mu$ ). Ambos estudios administraron el tratamiento durante 13 ciclos e incluyeron a 2152 (23) y a 2281 mujeres (25).

Tal y como se aprecia en la tabla, los resultados fueron muy variables, pero Calidad en todos los ensayos el número de embarazos fue similar entre los dos inbaja tervalos libres de hormonas comparados, y el índice de Pearl fue menor en el régimen de 24/4.

\begin{tabular}{|c|c|}
\hline Estudio & $\begin{array}{l}\text { Embarazos } \\
\text { (resultados mostrados para la comparación 24/4 frente 21/7) }\end{array}$ \\
\hline \multicolumn{2}{|l|}{ acetato de noretisterona } \\
\hline 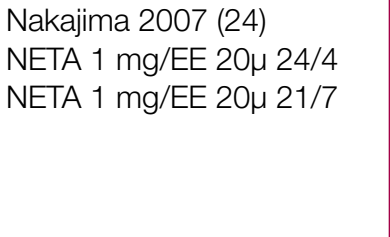 & $\begin{array}{l}\text { Número de embarazos: 0,7\% (5/705) frente 1,1\% (2/181) } \\
\text { (RR 0,64; IC95\% 0,12 a 3,28) } \\
\text { Î́ndice de Pearl: 1,82 (IC 95\% 0,59 a 4,25) frente 2,98 (IC } \\
\text { no descrito) } \\
\text { El riesgo acumulado de embarazo para el régimen 24/4 en } \\
\text { los primeros seis ciclos de uso fue del 0,9\% }\end{array}$ \\
\hline \multicolumn{2}{|l|}{ gestodeno } \\
\hline 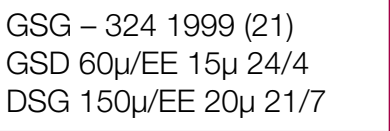 & $\begin{array}{l}\text { Número de embarazos: 0,18\% (1/539) frente 0,18\% (1/535) } \\
\text { (RR 0,99; IC95\% 0,06 a 15,83) }\end{array}$ \\
\hline \multicolumn{2}{|l|}{ drospirenona } \\
\hline 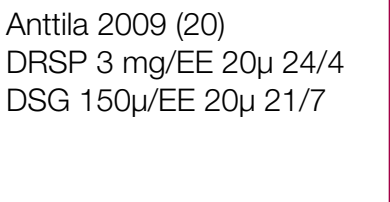 & $\begin{array}{l}\text { Número de embarazos: un único embarazo en el régimen } \\
\text { 21/7, atribuido por los autores a un fallo del método (HR de } \\
\text { embarazo 0,0045 (IC95\% 0,0006 a 0,0318)). } \\
\text { Índice de Pearl: } 0 \text { (extremo superior IC95\% 3,40) frente } \\
\text { 0,93 (extremo superior IC95\% 5,16) }\end{array}$ \\
\hline 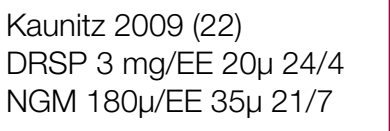 & $\begin{array}{l}\text { Número de embarazos: un único embarazo en el régimen } \\
21 / 7\end{array}$ \\
\hline \multicolumn{2}{|l|}{ acetato de nomegestrol } \\
\hline $\begin{array}{l}\text { Mansour } 2011 \text { (23) } \\
\text { NOMAC } 2,5 \text { mg/EE 1,5 mg } \\
\text { DRSP } 3 \text { mg/EE } 20 \mu\end{array}$ & $\begin{array}{l}\text { Número de embarazos: 0,2\% (4/1587) frente 0,5\% } \\
(3 / 534) \text { (RR 0,45; IC95\% 0,10 a 1,99) } \\
\text { Índice de Pearl: 0,31 (IC 95\% 0,08 a 0,79) frente 0,66 } \\
\text { (IC95\% 0,14 a 1,94) } \\
\text { El riesgo acumulado de embarazo tras un año de uso fue } \\
\text { del 0,33\% (IC95\% 0,12 a 0,87) frente 0,64\% } \\
\text { (IC95\% 0,21 a 1,97) }\end{array}$ \\
\hline $\begin{array}{l}\text { Westhoff } 2012 \text { (25) } \\
\text { NOMAC } 2,5 \text { mg/EE } 1,5 \mathrm{mg} \\
\text { DRSP } 3 \text { mg/EE } 20 \mu\end{array}$ & $\begin{array}{l}\text { Índice de Pearl: 1,27 (IC 95\% 0,66 a 2,22) frente 1,89 } \\
\text { (IC95\% 0,69 a 4,11) } \\
\text { El riesgo acumulado de embarazo tras un año de uso fue } \\
\text { del 1,22\% (IC95\% 0,69 a 2,16) frente 1,82\% } \\
\text { (IC95\% 0,81 a 4,05) }\end{array}$ \\
\hline
\end{tabular}


Los resultados sobre el cumplimiento con el tratamiento también fueron similares entre los dos regímenes de intervalos libres de hormonas en los estudios valorados, sin detectar en ningún caso diferencias significativas entre los regímenes de 24/4 y 21/7.

\begin{tabular}{|c|c|}
\hline Estudio & $\begin{array}{l}\text { Cumplimiento (resultados mostrados } \\
\text { para la comparación } 24 / 4 \text { frente } 21 / 7 \text { ) }\end{array}$ \\
\hline \multicolumn{2}{|l|}{ acetato de noretisterona } \\
\hline $\begin{array}{l}\text { Nakajima } 2007 \text { (24) } \\
\text { NETA } 1 \mathrm{mg} / \mathrm{EE} 20 \mu \mathrm{24/4} \\
\text { NETA } 1 \mathrm{mg} / \mathrm{EE} 20 \mu 21 / 7\end{array}$ & $\begin{array}{l}\text { Cumplimiento: } 77,2 \% \text { frente } 75,4 \% \\
\text { Interrupción del tratamiento por efectos adversos: } 6,2 \% \\
\text { frente } 7 \% \\
\text { Interrupción del tratamiento por falta de eficacia: } 0,5 \% \\
\text { frente } 1,1 \%\end{array}$ \\
\hline \multicolumn{2}{|l|}{ gestodeno } \\
\hline $\begin{array}{l}\text { GSG }-3241999(21) \\
\text { GSD } 60 \mu / E E 15 \mu 24 / 4 \\
\text { DSG 150 } \mu / E E ~ 20 \mu 21 / 7\end{array}$ & $\begin{array}{l}\text { Interrupción del tratamiento: } 64 / 539 \text { frente 51/535; RR } \\
1,25 \text { (IC95\% 0,88 a 1,76) }\end{array}$ \\
\hline \multicolumn{2}{|l|}{ drospirenona } \\
\hline $\begin{array}{l}\text { Anttila } 2009 \text { (20) } \\
\text { DRSP } 3 \text { mg/EE } 20 \mu 24 / 4 \\
\text { DSG } 150 \mu / E E ~ 20 \mu 21 / 7\end{array}$ & $\begin{array}{l}\text { Cumplimiento: de un número máximo de } 28 \text { píldoras, el } \\
\text { número de píldoras tomadas por ciclo osciló entre las } 26,5 \\
\text { y } 27,7 \text { frente a } 20,8 \text { a } 21,1\end{array}$ \\
\hline $\begin{array}{l}\text { Kaunitz } 2009 \text { (22) } \\
\text { DRSP } 3 \text { mg/EE } 20 \mu 24 / 4 \\
\text { NGM } 180 \mu / \text { EE } 35 \mu 21 / 7\end{array}$ & Interrupción del tratamiento: $2 / 167$ frente $1 / 165$ \\
\hline \multicolumn{2}{|l|}{ acetato de nomegestrol } \\
\hline $\begin{array}{l}\text { Mansour } 2011 \text { (23) } \\
\text { NOMAC 2,5mg/EE 1,5 mg } \\
\text { DRSP } 3 \text { mg/EE } 20 \mu\end{array}$ & Cumplimiento: $94,8 \%$ frente $91,4 \%$ \\
\hline
\end{tabular}

\subsection{Tromboembolismo}

\subsubsection{Tromboembolismo venoso y arterial}

Se identificaron cuatro revisiones sistemáticas que evaluaron el riesgo de enfermedad tromboembólica venosa, de ictus y de infarto de miocardio, asociados al uso de anticonceptivos hormonales (27-31).

La revisión más reciente (30), publicada también como revisión sistemática Cochrane (31) es un metanálisis de comparaciones múltiples que evaluó el riesgo de enfermedad tromboembólica venosa asociado al uso de anticonceptivos hormonales orales (fecha de búsqueda abril de 2013).

La revisión incluyó 26 estudios observacionales (estudios de cohorte, casos y controles y casos y controles anidados) y tuvo como desenlace principal el primer evento tromboembólico, fatal o no fatal. El metanálisis en red se desarrolló a partir de una exten- 
sión del modelo de efectos aleatorios frecuentista para comparaciones mixtas de múltiples tratamientos. Debido a que los autores no dispusieron de los datos crudos de los estudios, no se pudieron ajustar los análisis a factores de confusión.

La revisión de Peragallo Urrutia 2013 (29) incluyó 50 estudios observacionales que evaluaron el riesgo de enfermedad tromboembólica venosa, ictus e infarto de miocardio asociado al uso de anticonceptivos hormonales orales (búsqueda de junio de 2012). Los resultados de los estudios originales se combinaron con un modelo de efectos aleatorios.

En una revisión sistemática de miembros de la Fundación Española de Contracepción con unos objetivos similares a las otras dos revisiones comentadas ((28), fecha de búsqueda de abril 2010), se incluyeron 25 estudios observacionales y se describieron estimadores del efecto que compararon diferentes tipos de anticonceptivos orales de manera directa.

Por último, una revisión sistemática ((27), fecha de búsqueda de diciembre de 2011) incluyó tres estudios de cohortes y cinco estudios de casos y controles sobre el riesgo de enfermedad tromboembólica venosa asociado al uso de anticonceptivos de sólo gestágeno.

\subsubsection{Usuarias de anticonceptivos hormonales orales}

El metanálisis en red de Stegeman 2013 (30) recogió datos sobre la incidencia de eventos tromboembólicos en mujeres no usuarias de anticonceptivos hormonales de dos cohortes incluidas en su revisión de la literatura $(32,33)$. La incidencia fue de 1,9 y de 3,7 por cada 10.000 mujeres/año, dato similar a la de otros estudios en la literatura en que varían entre 1 y 6 casos por cada 10.000 mujeres/año.

Teniendo en cuenta este dato en población no usuaria de anticonceptivos hormonales orales, el uso de estos anticonceptivos aumenta el riesgo de sufrir un evento tromboembólico hasta tres veces (15 estudios; RR 3,5; IC95\%

Calidad moderada $2,9$ a 4,3$)$.

El riesgo relativo de evento tromboembólico fue similar para los anticonceptivos combinados con 30 a $35 \mu \mathrm{g}$ de etinilestradiol (EE) y gestodeno, desogestrel, acetato de ciproterona, o drospirenona. El riesgo en términos relativos de estos anticonceptivos fue entre el 50 y el $80 \%$ superior al riesgo asociado al uso de anticonceptivos combinados con levonorgestrel.

Basados en los resultados de 25 estudios, al compararlos con las no usuarias, los autores describen que los anticonceptivos hormonales orales con gestágenos de primera generación aumentan el riesgo hasta tres veces de sufrir un evento tromboembólico (RR 3,2; IC95\% 2 a 5,1), 2,8 veces en relación a los anticonceptivos con gestágenos de segunda generación (IC95\% 2 a 4,1), y 3,8 veces en relación a los de tercera generación (IC95\% 2,7 a 5,4).

El riesgo de sufrir un evento tromboembólico fue similar al comparar los anticonceptivos hormonales orales con gestágenos de primera y segunda generación (RR 0,9; IC95\% 0,6 a 1,4), pero en cambio las usuarias de anticonceptivos con gestágenos de tercera generación mostraron un mayor riesgo que las usuarias de anticonceptivos de segunda generación (RR 1,3; IC95\% 1,0 a 1,8). 
Se compararon los datos sobre el riesgo de sufrir un evento tromboembólico en 14 estudios, que mostraron una relación dosis y respuesta para el gestodeno, desogestrel, y el levonorgestrel que incrementaron el riesgo de un evento tromboembólico a mayores dosis de EE. Las combinaciones de dosis de $35 \mu \mathrm{g}$ EE y acetato de ciproterona $(0,90 ;$ IC95\% 0,6 a 1,3) y de $30 \mu \mathrm{g}$ EE y drospirenona (0,9; IC95\% 0,7 a 1,3) mostraron un riesgo similar a los $30 \mu \mathrm{g}$ EE y desogestrel.

Las comparaciones realizadas en la revisión de Martínez 2012 (28) mostraron que los anticonceptivos hormonales con menor riesgo de eventos tromboembólicos fueron aquellos con norgestimato o levonorgestrel. No se observaron diferencias significativas al comparar estos dos gestágenos (4 estudios, OR 1,11; IC95\% 0,84 a 1,46). El gestodeno (5 estudios, OR 1,33; IC95\% 1,08 a 1,63), el desogestrel (8 estudios, OR 1,93; IC95\% 1,31 a 2,83), la drospirenona (4 estudios, OR 1,67; IC95\% 1,10 a 2,55), y el acetato de ciproterona (6 estudios; OR 1,65; IC95\% 1,30 a 2,11), mostraron un mayor riesgo de evento tromboembólico que el levonorgestrel

La revisión de Peragallo Urrutia 2013 (29) ofrece datos de 15 estudios en los que se evaluó el riesgo de sufrir un ictus con el uso de anticonceptivos hormonales orales. Los anticonceptivos hormonales orales aumentaron el riesgo de ictus isquémico al compararlos con las no usuarias de anticonceptivos (7 estudios, OR 1,90; IC95\% 1,24 a 2,91), pero no de ictus hemorrágico (4 estudios, OR 1,03; IC95\% 0,71 a 1,49).

Los resultados de ocho estudios tampoco mostraron un aumento del riesgo de infarto de miocardio entre las usuarias de anticonceptivos orales y las no usuarias (OR 1,34; IC95\% 0,87 a 2,08).

Previo a la revisión sistemática de Peragallo Urrutia 2013 (29) se han publicado los datos de una cohorte poblacional sobre el riesgo de ictus e infarto de miocardio asociado al uso de anticonceptivos hormonales (34). El estudio aportó datos de un millón y medio de usuarias de anticonceptivos hormonales, entre los 15 y 49 años, no embarazadas y sin antecedentes de enfermedad cardiovascular o cáncer, en las que se registraron 21,4 ictus por cada 100000 usuarias/año, y 10,1 infartos de miocardio por cada 100000 usuarias/año.

Comparado con el riesgo en mujeres que no usaban anticonceptivos se observó un incremento del riesgo de ictus e infarto de miocardio que varió dependiendo de la dosis de EE y el tipo de gestágeno del anticonceptivo. La siguiente tabla detalla los resultados de la cohorte, marcando con un asterisco los estimadores que sugirieron un aumento de riesgo significativo: 


\begin{tabular}{|c|c|c|c|c|}
\hline & \multicolumn{2}{|c|}{ Ictus } & \multicolumn{2}{|c|}{ Infarto de miocardio } \\
\hline & EE 20 $\mu \mathrm{g}$ & EE 30 a $40 \mu \mathrm{g}$ & EE 20 $\mu \mathrm{g}$ & EE 30 a $40 \mu g$ \\
\hline Noretindrona & - & $2,2(1,5 \text { a } 3,2)^{\star}$ & - & $2,3(1,3 \text { a } 3,9)^{*}$ \\
\hline Levonorgestrel & - & $1,7(1,4 \text { a } 2,0)^{*}$ & - & $2,0(1,6 \text { a } 2,5)^{\star}$ \\
\hline Norgestimato & - & $1,5(1,2 \text { a } 1,9)^{*}$ & - & $1,3(0,9$ a 1,9$)$ \\
\hline Desogestrel & $1,5(1,3 \text { a } 1,9)^{\star}$ & $2,2(1,8 \text { a } 2,7)^{\star}$ & $1,6(1,1 \text { a } 2,1)^{\star}$ & $2,1(1,5 \text { a } 2,8)^{\star}$ \\
\hline Gestodeno & $1,7(1,4 \text { a } 2,1)^{\star}$ & $1,8(1,6 \text { a } 2,0)^{*}$ & $1,2(0,8$ a 1,9$)$ & $1,9(1,6 \text { a } 2,3)^{*}$ \\
\hline Drospirenona & $0,9(0,2$ a 3,5$)$ & $1,6(1,2 \text { a } 2,2)^{\star}$ & - & $1,7(1,0 \text { a } 2,6)^{\star}$ \\
\hline
\end{tabular}

\subsubsection{Usuarias de parche transdérmico y anillo vaginal}

La revisión de Martínez 2012 (28) recogió los resultados de tres estudios de casos y controles que mostraron un riesgo de evento tromboembólico similar entre el norgestimato y el parche transdérmico de norelgestromina (OR 1,18; IC95\% 0,73 a 1,89).

En la cohorte poblacional sobre el riesgo de ictus e infarto de miocardio asociado al uso de anticonceptivos hormonales (34), el parche transdérmico se asoció a un aumento no significativo del riesgo de sufrir un ictus comparado con las no usuarias de anticonceptivos hormonales (RR 3,2; IC95\% 0,8 a 12,6), sin observar casos de infarto de miocardio.

Por otro lado, en esta cohorte se observó un aumento significativo del riesgo de ictus entre las usuarias del anillo vaginal, al compararlo con el de las no usuarias de anticonceptivos hormonales (RR 2,5; IC95\% 1,4 a 4,4), pero no de infarto de miocardio (RR 2,1; IC95\% 0,7 a 6,5).

En otro estudio de una cohorte prospectiva que incluyó usuarias de Estados Unidos y varios países europeos se evaluó el riesgo cardiovascular de 16.864 usuarias del anillo (35). En esta cohorte se observó una incidencia de 8,3 tromboembolismos venosos por cada 10000 usuarias/año (IC95\% 5 a 12,9 casos por cada 10000 usuarias/año), sin que se observaran diferencias con el riesgo de tromboembolismo de los anticonceptivos hormonales orales (HR 0,8; IC95\% 0,5 a 1,5).

\subsubsection{Usuarias de anticonceptivos de sólo gestágeno}

Ninguno de los ocho estudios incluidos en la revisión sistemática de Mantha 2012 (27), mostró que los anticonceptivos de sólo gestágeno aumenten el Calidad riesgo de un evento tromboembólico venoso al compararlos con los datos baja de las mujeres no usuarias de contracepción. Un estimador del efecto combinado de estos estudios confirmó los resultados de los estudios individuales (RR 1,03; IC95\% 0,76 a 1,39).

Calidad moderada

Calidad baja 
Aunque no se detectó un aumento del riesgo entre las usuarias de anticonceptivos de sólo gestágeno orales (RR 0,90; IC95\% 0,57 a 1,45) o intrauterinos (RR 0,61; IC95\% 0,24 a 1,53), los datos de dos estudios sugirieron el aumento del doble de riesgo de un evento tromboembólico entre las usuarias del anticonceptivo inyectable y las no usuarias (RR 2,67; IC95\% 1,29 a 5,53). No obstante estos dos estudios tenían una muestra limitada (n:360) e incluyeron a mujeres con riesgo de sufrir este tipo de eventos.

Pese a que la literatura disponible muestra de manera consistente que los anticonceptivos hormonales combinados aumentan el riesgo de enfermedad tromboembólica venosa, es importante destacar que en la elección de un anticonceptivo hormonal deben tenerse en cuenta tanto los datos reflejados en la literatura como las características de cada usuaria y su posible riesgo de enfermedad tromboembólica. Por este motivo es importante interpretar los resultados de la literatura en términos absolutos. En este sentido la revisión de Martínez 2012 (28), mostró una estimación en términos absolutos del riesgo de enfermedad tromboembólica venosa al comparar el uso de anticonceptivos hormonales con las de mujeres en otras etapas de sus vidas.

Por otro lado la Agencia Española de Medicamentos y Productos Sanitarios elaboró una revisión del riesgo de tromboembolismo venoso asociado a los anticonceptivos hormonales combinados (36). Sus conclusiones destacan que: i) el beneficio de estos anticonceptivos en la prevención de embarazos no deseados continúa siendo superior a los posibles riesgos asociados a su uso; ii) los datos confirman que el riesgo conocido de tromboembolismo venoso es bajo, pero que aquellos que contienen levonorgestrel, noretisterona o norgestimato como progestágenos presentan un riesgo más bajo; iii) la probabilidad de tromboembolismo venoso es mayor durante el primer año de uso, al reiniciarlo tras una interrupción de al menos 4 semanas y en mujeres que presentan factores de riesgo (mayor edad, tabaquismo, sobrepeso, migrañas, historia familiar de tromboembolismo o parto reciente); iv) sobre el tromboembolismo arterial no se dispone de información suficiente que permita establecer diferencias entre los distintas combinaciones.

Tanto la revisión de Martínez 2012 (28) como el informe de la AEMPS recogen la estimación del riesgo de tromboembolismo venoso asociado a los diferentes tipos de anticonceptivos hormonales. En Martínez 2012 (28) los datos de referencia para estas estimaciones se calcularon a partir de los resultados de la revisión y de los datos de un estudio poblacional (15), marcados con un asterisco en la tabla. Los datos recogidos en el informe de la AEMPS no identifican su referencia y se identifican en la tabla con una $\operatorname{cruz}(\dagger)$. 


\begin{tabular}{|l|c|c|c|}
\hline & $\begin{array}{c}\text { Riesgo relativo de } \\
\text { ETV }\end{array}$ & \multicolumn{2}{|c|}{$\begin{array}{c}\text { Riesgo en términos absolutos por } \\
\text { cada } \mathbf{1 0 0 0 0} \text { mujeres/año }\end{array}$} \\
\hline $\begin{array}{l}\text { No usuarias - } \\
\text { No embarazadas }\end{array}$ & 1 & $2,3(3 \text { a } 13,3)^{\star}$ & $2 \dagger$ \\
\hline Levonorgestrel & $1,33(1,08-1,63)$ & $10,6(6,9$ a 15,6$)$ & 9 a $12 \dagger$ \\
\hline Gestodeno & $1,93(1,31-2,85)$ & $15,4(10,0$ a 22,5$)$ & 9 a $12 \dagger$ \\
\hline Desogestrel & $1,67(1,10-2,55)$ & $13,3(7,02$ a 24,4$)$ & 9 a $12 \dagger$ \\
\hline Drospirenona & $1,65(1,30-2,11)$ & $13,2(8,6$ a 19,3$)$ & \\
\hline Acetato de ciproterona & & $29,4(6,0 \text { a } 82,0)^{\star}$ & \\
\hline Embarazadas & & & \\
\hline
\end{tabular}

\section{De la evidencia a la recomendación}

\section{Resumen de la evidencia}

Los métodos anticonceptivos hormonales que han mostrado una mayor eficacia son aquellos con acción prolongada (implantes y DIU de levonorgestrel) con una incidencia de fallo del método durante el primer año asociada al uso habitual del 0,08 y 0,1 por cada 100 mujeres/año respectivamente. La eficacia mostrada por estos anticonceptivos es similar a los de la esterilización femenina.

A estos dos métodos los siguen los métodos: píldora de progestágeno solo (incidencia del 0,41), anillo (incidencia del 1,23), parche (incidencia del 1,24), y anticonceptivos de acción corta (anticonceptivos orales (incidencia del 2,1).

El uso de anticonceptivos hormonales orales combinados se asocia con un aumento del riesgo de sufrir un evento tromboembólico venoso. La magnitud en términos relativos de este riesgo depende del tipo de gestágeno y de la dosis de etinilestradiol (30). Aquellos anticonceptivos que contienen levonorgestrel, noretisterona o norgestimato como progestágenos presentan un riesgo más bajo (36).

El uso de anticonceptivos hormonales orales se asocia con un aumento del riesgo de ictus isquémico, pero no de ictus hemorrágico o infarto de miocardio (29).

Una cohorte poblacional mostró un aumento no significativo del riesgo de sufrir un ictus entre las usuarias del parche, al compararlo con el riesgo de las mujeres no usuarias de anticonceptivos hormonales (34). No se han observado diferencias en el riesgo de enfermedad tromboembólica venosa entre los anticonceptivos con norgestimato y el parche transdérmico de norelgestromina (28).

Calidad moderada

Calidad moderada

Calidad moderada

Calidad baja

Calidad moderada 
Las usuarias del anillo vaginal han mostrado un aumento significativo de ictus frente a las mujeres que no usan anticonceptivos hormonales (34). El riesgo de tromboembolismo venoso es bajo, sin que haya diferencias con el Calidad baja riesgo de las usuarias de anticonceptivos hormonales orales (35).

Los anticonceptivos de sólo gestágeno en su conjunto no aumentan el riesgo de enfermedad tromboembólica venosa en comparación con las no usuarias de anticonceptivos. Los limitados datos disponibles sobre los anticonceptivos inyectables de sólo gestágeno han mostrado una asociación con el doble de riesgo de enfermedad tromboémbolica venosa comparado con las mujeres no usuarias (27).

\section{Calidad de la evidencia}

La calidad global es moderada.

Para los anticonceptivos hormonales e intrauterinos se ha bajado la calidad de la evidencia en sólo un grado por considerar que la inclusión de estudios observacionales puede aportar ciertos sesgos a las estimaciones de los resultados. La variabilidad de las características de los estudios y sus resultados aporta cierta heterogeneidad a las estimaciones disponibles, aunque parecen ser consistentes entre los estudios.

Con relación a los datos disponibles sobre el riesgo de tromboembolismo venoso, la mayoría de los estudios disponibles son observacionales con limitaciones en su diseño o resultados que en ocasiones son inconsistentes, por lo que en términos generales la calidad de la evidencia es baja. En el caso del riesgo de tromboembolismo en usuarias de anticonceptivos hormonales orales, se considera que la calidad es moderada ya que los resultados disponibles para los anticonceptivos hormonales orales han mostrado una gran consistencia, confirmada por el modelo de comparaciones múltiples del metanálisis en red de Stegeman 2013 (30).

\section{Valores y preferencias de las usuarias}

Una revisión sistemática sobre los aspectos más comunes incluidos en las herramientas de decisión compartida en anticoncepción y en los estudios que evalúan las preferencias de las usuarias de anticonceptivos mostró que los aspectos de seguridad fueron los más destacados (37). En este sentido, el uso de información personalizada a las futuras usuarias de anticonceptivos se ha asociado en un ensayo clínico a la elección de métodos anticonceptivos eficaces (38), aunque los estudios sobre estrategias de comunicación sobre la eficacia de los anticonceptivos no han evaluado adecuadamente la percepción sobre su seguridad y su influencia sobre los valores y las preferencias de las mujeres (39).

En las conclusiones de su revisión del riesgo de tromboembolismo asociado al uso de anticonceptivos hormonales combinados, la AEMPS destaca la importancia de informar a las usuarias sobre el beneficio derivado del uso de estos anticonceptivos y del riesgo en términos absolutos de tromboembolismo, así como de los signos y síntomas de alerta que permitan su diagnóstico precoz (36). 


\section{Beneficios y riesgos/carga de las intervenciones}

No se ha considerado este aspecto en esta pregunta clínica.

\section{Balance beneficio/riesgo}

La efectividad de los anticonceptivos hormonales combinados es similar entre los diferentes tipos disponibles, por lo que es imprescindible conocer el riesgo de tromboembolismo individual de cada mujer cuando se decide iniciar el uso de un anticonceptivo hormonal.

La revisión de Stegeman 2013 (30) muestra que el mayor riesgo se ha observado entre las usuarias de etinilestradiol de $50 \mu \mathrm{g}$ con levonorgestrel, y etinilestradiol de $35 \mu \mathrm{g}$ con acetato de ciproterona, etinilestradiol de $30 \mu \mathrm{g}$ con drospirenona o etinilestradiol de $30 \mu \mathrm{g}$ con desogestrel, con un riesgo similar. El menor riesgo se ha observado entre las usuarias de levonorgestrel 30 ó $20 \mu \mathrm{g}$, gestodeno de $20 \mu \mathrm{g}$, o norgestimato $35 \mu \mathrm{g}$ (30). El riesgo relativo de evento tromboembólico fue similar para los anticonceptivos con 30 a $35 \mu \mathrm{g}$ de etinilestradiol y gestodeno, desogestrel, acetato de ciproterona, o drospirenona, pero fue entre el 50 y el $80 \%$ superior al riesgo asociado al uso de anticonceptivos con levonorgestrel. Por otro lado, no se han observado diferencias en el riesgo de enfermedad tromboembólica venosa entre los anticonceptivos norgestimato oral y el parche transdérmico de norelgestromina (28).

En las mujeres con un mayor riesgo de enfermedad tromboémbólica puede ser pertinente considerar el uso de anticonceptivos de sólo gestágeno no inyectables (27).

Cuando se discute con las mujeres sobre este aspecto, es importante destacar la eficacia y efectividad de los anticonceptivos y transmitir cuál puede ser el riesgo de sufrir un evento tromboémbolico en términos absolutos (ver tabla Martínez 2012 en la descripción de los resultados de la literatura (28)).

\section{Uso de recursos y costes}

Se ha identificado un estudio de coste beneficio que comparó cuatro métodos anticonceptivos hormonales: i) inyectable de 17-acetato de medroxiprogesterona (DMPA); ii) implante subdérmico de levonorgestrel; iii) anticonceptivo oral de gestágeno solo; y iv) anticonceptivo oral combinado (AOC) (40). Los análisis se realizaron para estimar los embarazos evitados mediante un modelo sobre una cohorte hipotética de mujeres estadounidenses de entre 18 y 44 años de edad sin complicaciones de salud relevantes tomando como referencia precios del año 1992. En este estudio el anticonceptivo oral de gestágeno solo y el AOC mostraron la mayor tasa de fallo en el primer año de uso (5\%), tasa superior al $0,3 \%$ de los otros dos métodos. Entre las usuarias del implante se estimó que hasta el $60 \%$ abandonarían el método en los primeros 5 años de uso. Según los autores, el método inyectable fue el método con un mayor coste-beneficio, con un beneficio por usuaria de 2,87 para el método inyectable, 2,79 \$ para el anticonceptivo oral de gestágeno solo, 2,77 \$ para el anticonceptivo oral combinado y 1,64\$ para el implante. No obstante el estudio mostró serias limitaciones en la definición de las fuentes de sus datos de eficacia y costes de referencia.

Un estudio de coste beneficio posterior muy similar recopiló datos de eficacia de estudios publicados hasta 1994 y datos de referencia de costes del periodo 1991 a 1993 
para una cohorte hipotética de mujeres estadounidenses. Este estudio comparó el coste beneficio de 15 métodos hormonales y no hormonales (41). Las tasas de fallo más bajas las registró la vasectomía $(0,04 \%)$, mientras que el implante y el inyectable DMPA mostraron una tasa del $0,3 \%$, y los anticonceptivos orales del 3\%. El modelo económico con una perspectiva de 5 años mostró que el DIU de cobre, la vasectomía y el implante eran las alternativas más ventajosas desde un modelo de servicios de salud privados, aportando un ahorro alrededor de los 14 dólares estadounidenses y previniendo 4,2 embarazos por persona. De todos modos los autores pusieron de manifiesto que el precio directo de adquisición de los anticonceptivos no es un indicador adecuado dado que la anticoncepción deriva en un ahorro económico y social al prevenir embarazos no deseados.

Un estudio de coste utilidad ha comparado el impacto neto sobre la salud y económico de 13 métodos anticonceptivos hormonales y no hormonales frente a no usar anticonceptivos con una perspectiva social en el modelo económico (42). Como en el estudio anterior se tomaron datos sobre la eficacia de los métodos a partir de estudios publicados hasta 2004 y se realizó una simulación mediante un modelo de Markov y Monte Carlo, tomando como referencia precios del año 2002 y con un horizonte temporal de 2 años. Se evaluaron multitud de desenlaces de interés relacionados con la eficacia de los diferentes métodos, el riesgo de infección de transmisión sexual (ITS) y los efectos adversos. Los diferentes métodos evaluados mostraron una media de años ganados por calidad de vida (AVAC o QALY) favorable entorno a los 1,9 AVAC. Cuando se compararon los métodos dependiendo del coste extra que supone la ganancia de un AVAC, el método inyectable DMPA mostró una relación de coste-efectividad incremental (ICER) de 18,406\$ frente a la vasectomía. El resto de comparaciones estuvieron dominadas por el DMPA, dado que mostraron un mayor coste con un beneficio menor. Todos los métodos mostraron un ahorro significativo frente a no usar anticonceptivos (de hasta 780\$) incluso en un escenario restringido a un año de uso. En definitiva, el uso de anticonceptivos se mostró favorable independientemente del método frente al no uso en términos de beneficio para la salud y ahorro económico, siendo los métodos que requieren de un uso menos regular los que mostraron un mayor beneficio y menor coste.

Otro análisis de coste-efectividad, evaluó si los métodos de acción prolongada (LARC por su acrónimo en inglés) eran más coste eficaces que otros métodos anticonceptivos (píldora o esterilización femenina) en la prevención de embarazos no deseados (43). La evaluación se basó en un modelo de decisión de Markov con un horizonte temporal de 15 años con la perpectiva del sistema sanitario británico (NHS), comparando los dispositivos intrauterinos, el implante y el método inyectable. Los costes que se consideraron fueron los derivados del uso de los anticonceptivos y los asociados a un nacimiento fruto de un embarazo no deseado. Entre los dos y 15 años de uso, los métodos LARC fueron los anticonceptivos más eficaces y menos costosos. Durante los primeros cuatro años de uso la esterilización femenina supuso una relación de coste incremental de 38197 libras por embarazo evitado en relación a los LARC, sobrecoste que se atenuó en el tiempo y se invirtió a partir del sexto año de uso. El método LARC menos dominante fue el inyectable. El implante supuso una relación de coste incremental de 13206 libras por embarazo evitado frente a los DIU, circunstancia que se invirtió a los 15 años de uso. A la luz de estos datos, los autores del estudio concluyeron que los métodos LARC son coste eficaces, aunque deben identificarse métodos para mejorar la aceptabilidad y evitar la interrupción del uso. 
Por último, otro estudio de coste utilidad posterior usó una metodología similar a la del estudio anterior para comparar 16 métodos anticonceptivos hormonales y no hormonales en población estadounidense frente a no usar anticonceptivos, en un modelo de Markov con un horizonte temporal de 5 años. Los autores concluyeron que los métodos más coste efectivos eran el DIU de cobre, la vasectomía y el DIU liberador de levonorgestrel (DIU-LNG), aunque los resultados dependían del coste estimado derivado del embarazo no deseado y de la perspectiva temporal en la que se realizaba el análisis. Los costes derivados de los diferentes métodos en un horizonte temporal de 5 años fueron: 647 para el DIU de cobre; 713 \$ para la vasectomía; y 930\$ para el DIU-LNG. El resto de anticonceptivos hormonales tenían un coste mayor: $1,597 \$$ con el implante; $2,681 \$$ con el inyectable; $3,381 \$$ con los AO; y 3,458\$ con el parche. Todos los métodos anticonceptivos se mostraron más eficaces que no usarlos. El DIU de cobre se mostró como la alternativa dominante al mostrar un incremento en la relación de coste-efectividad incremental (ICER) de $164 \$$ frente a la vasectomía. El resto de alternativas mostraron un ICER mayor: en el caso del DIU-LNG fue de 1,415\$ frente a la vasectomía, y el implante fue de $2,375 \$$ frente al DIU-LNG (44).

\section{Justificación}

Las recomendaciones en este apartado derivan de datos en la literatura que, pese a tener algunas limitaciones, muestran resultados consistentes. El beneficio derivado de los métodos anticonceptivos se ha mostrado regular a lo largo del tiempo. El Grupo de Trabajo priorizó la importancia de los resultados de efectividad (uso habitual) de los anticonceptivos hormonales e intrauterinos para graduar la recomendación, y los datos de los estudios económicos que muestran que pueden ofrecer un perfil de coste-beneficio favorable, no sólo derivados del coste directo de los anticonceptivos sino del beneficio económico y social derivado de los embarazos no deseados.

Por otro lado, el Grupo de Trabajo destacó la necesidad de reflejar las conclusiones de la revisión del riesgo de tromboembolismo venoso asociado a los anticonceptivos hormonales combinados de la Agencia Española de Medicamentos y Productos Sanitarios (36).

Las recomendaciones en este apartado se alinean con las de la Estrategia de Salud Sexual y Reproductiva del Sistema Nacional de Salud (SNS) para la mejora de la accesibilidad universal a las prácticas clínicas efectivas de planificación de la reproducción, mediante la incorporación en la cartera de servicios comunes del SNS de anticonceptivos que hayan mostrado su eficacia en la literatura científica (de acuerdo a la Ley Orgánica 2/2010) (1). Se incorpora la necesidad de individulizar la decisión a las circunstancias de las usuarias como una manera de considerar aspectos que determinen el uso y beneficio del método anticonceptivo y puedan identificar contextos de vulnerabilidad y evitar situaciones que contribuyan a la inequidad en la prestación de servicios. 


\section{Recomendaciones}

\begin{tabular}{|l|l|}
\hline Fuerte & $\begin{array}{l}\text { Se recomienda el uso de anticonceptivos hormonales e intrauterinos, debido } \\
\text { a que han mostrado una elevada efectividad. En la elección de un anticon- } \\
\text { ceptivo cada profesional sanitario debe considerar las circunstancias y pref- } \\
\text { erencias de cada mujer. }\end{array}$ \\
\hline Fuerte & $\begin{array}{l}\text { Se recomienda que en la elección del anticonceptivo hormonal combinado } \\
\text { más adecuado cada profesional sanitario valore detalladamente los factores } \\
\text { de riesgo de tromboembolismo de la usuaria antes de iniciar el tratamiento } \\
\text { y periódicamente durante su uso. }\end{array}$ \\
\hline Fuerte & $\begin{array}{l}\text { Se recomienda que cada profesional sanitario informe a las usuarias sobre el } \\
\text { beneficio derivado del uso de los anticonceptivos hormonales combinados y } \\
\text { del riesgo en términos absolutos de tromboembolismo, así como de los sig- } \\
\text { nos y síntomas de alerta que permitan su diagnóstico precoz. }\end{array}$ \\
\hline $\begin{array}{l}\text { Buena } \\
\text { práctica } \\
\text { clínica }\end{array}$ & $\begin{array}{l}\text { Se sugiere la elaboración de protocolos para la provisión y manejo de an- } \\
\text { ticonceptivos en el marco del Sistema Nacional de Salud para asegurar la } \\
\text { actuación integral, y garantizar la accesibilidad universal a los métodos an- } \\
\text { ticonceptivos. }\end{array}$ \\
\hline
\end{tabular}




\section{Anticoncepción de urgencia}

Preguntas a responder:

- ¿Cuál es la eficacia de los anticonceptivos de urgencia?

- ¿Existen diferencias en términos de eficacia en la utilización de levonorgestrel frente al acetato de ulipristal como anticoncepción de urgencia?

- ¿Existen diferencias en términos de eficacia en la utilización de la anticoncepción de urgencia hormonal frente a la intrauterina?

- ¿La libre dispensación de la anticoncepción de urgencia de levonorgestrel tiene un impacto sobre la incidencia de infecciones de transmisión sexual, las conductas sexuales de riesgo o el uso incorrecto de la anticoncepción habitual?

\subsection{Eficacia y seguridad de la anticoncepción de urgencia}

Se han identificado datos sobre la eficacia de los métodos anticonceptivos de urgencia en una revisión sistemática Cochrane (fecha de búsqueda en julio de 2011) que tenía como objetivo determinar la eficacia y seguridad de los diferentes métodos de anticoncepción de urgencia tras una relación coital sin protección (45). La revisión evaluó métodos hormonales orales e intrauterinos y se focalizó en el número de embarazos y embarazos ectópicos como desenlaces de eficacia y los efectos adversos. Se evaluaron los resultados dependiendo del tiempo transcurrido desde la relación coital y dependiendo de si las mujeres habían mantenido o no otras relaciones coitales durante el ciclo en el que se administraba el anticonceptivo de urgencia.

La revisión incluyó 100 ensayos clínicos. A parte de dos estudios de la OMS con amplias poblaciones de estudio, la gran mayoría de estudios -86- se publicaron en China a partir de la década de los 90. Principalmente los estudios compararon mifepristona frente a levonorgestrel, diferentes dosis de mifepristona o levonorgestrel entre ellas, y mifepristona o levonorgestrel frente al método de Yuzpe. Los estudios tuvieron un riesgo de sesgo considerable, puesto que solamente un cuarto de ellos ofreció una descripción adecuada del encubrimiento de la aleatorización, fueron doble ciego, u ofrecieron una descripción correcta de las pérdidas en el seguimiento.

La revisión de Cheng 2012 (45) también incluye un ensayo clínico de no inferiroridad simple ciego que comparó la eficacia y la seguridad del acetato de ulipristal frente el levonorgestrel para la anticoncepción de urgencia (46). Este estudio incluye un metanálisis con el ECA previo que evaluó la misma comparación de interés (47).

El ECA Glasier 2010 (46) incluyó a 2221 mujeres que solicitaron anticoncepción de urgencia durante los 5 días siguientes de una relación coital sin protección, reclutadas en 35 centros de planificación familiar en el Reino Unido, Irlanda y EEUU. Las mujeres 
fueron aleatorizadas a recibir de manera supervisada $30 \mathrm{mg}$ de acetato de ulipristal (n: 1104) o 1,5 mg de levonorgestrel (n: 1117). La mayoría de mujeres estuvo en la franja de edad entre los 21 y 35 años (63\%), solicitó anticoncepción de urgencia por una relación coital sin protección (55\%), que se produjo en una sola ocasión en el $89 \%$ de casos, entre las 0 y 72 horas tras la relación sin protección (hasta el $80 \%$ ). El desenlace principal del ensayo clínico fue la tasa de embarazo entre las mujeres que recibieron la anticoncepción de urgencia hasta las 72 horas posteriores de la relación coital sin protección. El estudio evaluó los resultados de las mujeres que finalmente recibieron la anticoncepción de urgencia (ulipristal 844 versus levonorgestrel 852) en un análisis de no inferioridad en el que se estableció un margen de diferencias entre los grupos del 1\%. Creinin 2006 comparó 50mg de ulipristal con $0,75 \mathrm{mg}$ de levonorgestrel tomada en dos dosis en un ECA de no inferioridad multicéntrico y doble ciego (47). El estudio evaluó los resultados de eficacia de 1549 mujeres (ulipristal 775 versus levonorgestrel 774) que recibieron el anticonceptivo hasta las 72 horas posteriores de la relación coital sin protección.

Estos dos estudios no tuvieron limitaciones destacables, puesto que aseguraron el encubrimiento de la aleatorización, fueron cegados y ofrecían suficiente información como para realizar análisis por intención de tratar.

Aunque la revisión Cochrane incluye los resultados de dos ensayos que compararon la eficacia del dispositivo intrauterino de cobre como método de anticoncepción de urgencia frente a conducta expectante (48) o mifepristona (49), otra revisión sistemática con unos criterios de inclusión más amplios identificó los estudios, independientemente de su diseño, que evaluaran algún dispositivo intrauterino (50).

Esta revisión incluyó 42 estudios de los que no se clarificó su diseño, que evaluaron un dispositivo intrauterino de cobre con la excepción de dos estudios que evaluaron el dispositivo Lippes Loop. Como en la revisión Cochrane, la mayoría de estudios se desarrollaron en China -28-. El dispositivo se insertaba en los 5 días siguientes a la relación coital sin protección en 31 de los estudios incluidos, aunque en tres estudios la inserción se realizaba hasta los 7 días, o en el caso de dos estudios hasta los 10 días o más. La inclusión de estudios no controlados en la revisión expone a sesgos importantes relacionados con el diseño de estos estudios, limitando la confianza en sus resultados.

Adicionalmente una guía de práctica clínica sobre la anticoncepción de urgencia elaborada por la unidad de evaluación Clinical Effectiveness Unit (CEU) de la Faculty of Sexual and Reproductive Healthcare y del Royal College of Obstetricians \& Gynaecologists (51), recoge recomendaciones relacionadas con el acetato de ulirpistal y el levonorgestrel en la anticoncepción de urgencia. También se ha recogido información sobre una guía para la provisión de anticoncepción de urgencia del European Consortium for Emergency Contraception (52), y un estándar de calidad elaborado por NICE (53). Cabe destacar, por otro lado, que en nuestro sistema sanitario el dispositivo intrauterino de levonorgestrel (DIU LNG) no dispone de indicación en ficha técnica como método de anticoncepción de urgencia (54), ni la mifepristona. 


\subsubsection{Eficacia de los anticonceptivos de urgencia}

El análisis combinado de 20 ensayos en la revisión de Cheng 2012 (45) mostró que la dosis de entre 25 y $50 \mathrm{mg}$ de mifepristona (20 ensayos; RR 0,64; IC $95 \%$ 0,45 a 0,92) o menor de 25 mg (11 ensayos; RR 0,70; IC95\% 0,50 a 0,97$)$ resulta en un número menor de embarazos que el levonorgestrel (LNG). Sin embargo un análisis de sensibilidad de los estudios con bajo riesgo de sesgo no mostró diferencias significativas (4 ensayos; RR 0,70; IC95\% 0,49 a 1,01).

De una manera similar, las dosis bajas de mifepristona fueron menos eficaces que las dosis de entre 25 y $50 \mathrm{mg}$ ( 25 ensayos; RR 0,73; IC95\% 0,55 a $0,97)$, aunque las diferencias desaparecieron tras analizar los ensayos con bajo riesgo de sesgo ( 6 ensayos; RR 0,75; IC95\% 0,50 a 1,10).

El LNG mostró una eficacia similar si se administra en una sola dosis de $1,5 \mathrm{mg}$ o en dos dosis de $0,75 \mathrm{mg}$ en un intervalo de 12 horas, independientemente del momento en que se administrara cualquiera de las dos pautas ( 3 ensayos; RR 0,84; IC95\% 0,53 a 1,33).

Los resultados combinados de 4 ensayos han mostrado que el LNG administrado dentro de las 72 horas siguientes a la relación coital sin protección reduce un número mayor de embarazos que si se administra con posterioridad (RR 0,51; IC95\% 0,31 a 0,84). En cambio el momento de la administración no pareció influir en el efecto de la mifepristona.

Por otro lado, el método de Yuzpe mostró una menor eficacia para prevenir el embarazo que la mifepristona (3 ensayos; RR 0,14; IC95\% 0,05 a 0,41) o el LNG (5 ensayos; RR 0,54; IC95\% 0,36 a 0,80), y un ensayo no

Calidad moderada

Calidad baja mostró diferencias entre la gestrinona y la mifepristona como método de anticoncepción de urgencia (RR 0,75; IC95\% 0,32 a 1,76).

\subsubsection{Eficacia del levonorgestrel frente al acetato de ulipristal como anticoncepción de urgencia}

El ECA de Glasier 2010 (46) mostró que el ulipristal presentó una menor tasa de embarazos (15; 1,8\% de las participantes) que el levonorgestrel (22;

Calidad moderada $2,6 \%$ de las participantes) aunque este resultado no supuso una diferencia significativa entre los grupos (1696 mujeres; OR 0,68; IC95\% 0,35 a 1,31). Estos resultados fueron muy similares cuando se evaluaron las mujeres que habían recibido el anticonceptivo entre las 72 y 120 horas posteriores a la relación coital sin protección (203 mujeres, 3 embarazos en el grupo de levonorgestrel; OR 0,57; IC95\% 0,29 a 1,09; P=0,091). 
Por su parte, el estudio de Creinin 2006 (47) mostró unos resultados similares: el ulipristal presentó una menor tasa de embarazos $(7 ; 0,9 \%$ de las participantes) que el levonorgestrel (13; 1,6\% de las participantes) sin que esta diferencia fuera significativa (1 ECA, 1549 mujeres; OR 0,50; IC95\% $0,18$ a 1,$24 ; \mathrm{P}=0,135)$.

Cuando se combinaron los resultados de ambos estudios se observó una menor tasa de embarazos entre las mujeres que habían recibido ulipristal entre 0 y 24 horas tras la relación sin protección (2 ECA, 5/584 embarazos ulipristal versus 15/600 embarazos levonorgestrel; OR 0,35; IC95\% 0,11 a 0,93). El ulipristal también mostró una menor tasa de embarazos que el levonorgestrel cuando se administró la anticoncepción de urgencia en las 120 horas posteriores al coito sin protección (2 ECA, 22/1714 embarazos ulipristal versus 38/1731 embarazos levonorgestrel; OR 0,55; IC95\% 0,32 a 0,93 ).

La diferencia entre ambos métodos de anticoncepción de urgencia fue marginal cuando se analizaron los resultados en las 72 horas siguientes al coito sin protección (2 ECA, 22/1617 embarazos ulipristal versus 35/1625 embarazos levonorgestrel; OR 0,58; IC95\% 0,33 a 0,99).

Por otro lado, la guía de práctica clínica sobre la anticoncepción de urgencia elaborada por la CEU de la Faculty of Sexual and Reproductive Healthcare (51) incluye las siguientes recomendaciones (con el máximo grado de recomendación) a partir del análisis de estos mismos estudios:

- La eficacia del acetato de ulipristal ha sido demostrada hasta 120 horas después de una relación sexual no protegida y puede ser ofrecido a mujeres que solicitan contracepción de urgencia durante este periodo de tiempo.

- La eficacia del levonorgestrel ha sido demostrada hasta las 96 horas después de una relación sexual no protegida. Su eficacia entre las 96 y 120 horas es desconocida.

\subsubsection{Seguridad de los anticonceptivos de urgencia}

Los análisis de los resultados de seguridad de la revisión de Cheng 2012 (45) mostraron que los anticonceptivos hormonales de urgencia fueron seCalidad guros en términos generales. Los métodos combinados con estrógenos se baja asocian a náuseas y vómitos, mientras que los métodos con progestágeno o con antiprogestágenos causaron cambios en menstruaciones posteriores. El retraso en la menstruación fue el principal efecto adverso asociado a la mifepristona y parece estar asociado a la dosis administrada. 


\subsubsection{Seguridad del levonorgestrel frente al acetato de ulipristal como anticoncepción de urgencia}

El ECA de Glasier 2010 (46) mostró una tasa de eventos adversos muy similar entre el ulipristal (597/1104; 54\% de las participantes) y el levonorgestrel (626/1117; 56\% de las participantes), que fueron evaluados como

Calidad baja leves o moderados en el 94\% de los casos (OR 0,92; IC 95\% 0,78 a 1,09).

El efecto adverso más frecuente fue la cefalea en el 20\% de las participantes. Sin llegar a la significación estadística en ningún caso, el ulipristal mostró una mayor tasa de cefalea, náuseas (15\%), fatiga (5\%), mareos (5\%) o lumbalgia (3\%). El levonorgestrel mostró una mayor tasa de dismenorrea $(15 \%)$ y dolor abdominal (10\%).

Por otro lado se han destacado como comunes los siguientes efectos adversos para el ulipristal y el levonorgestrel (55): cefalea (19\% en ambos), dismenorrea (13\% y $14 \%)$, náuseas $(13 \%$ y $11 \%)$, dolor abdominal $(5 \%$ y $7 \%$ ), mareos ( $5 \%$ en ambos), y fatiga (6\% y $5 \%$ ). También se destaca como común la alteración del ciclo menstrual normal en el caso del ulipristal, que puede ampliar la duración del ciclo menstrual en dos días.

Por otro lado la ficha técnica del acetato de ulipristal recoge los datos del informe de la EMA (56) sobre la posible pérdida de eficacia del método en mujeres con un peso corporal o IMC alto, aunque esta afirmación se basa en datos limitados y poco concluyentes derivados de reanálisis de los ensayos disponibles. El informe presenta los siguientes datos sobre la eficacia del método de acuerdo al IMC de las usuarias:

\begin{tabular}{|c|c|c|c|c|}
\hline IMC (kg/m $\mathbf{~})$ & $\begin{array}{c}\text { Bajo peso } \\
(\mathbf{0 - 1 8 , 5 )}\end{array}$ & $\begin{array}{c}\text { Normopeso } \\
(\mathbf{1 8 , 5 - 2 5 )}\end{array}$ & $\begin{array}{c}\text { Sobrepeso } \\
\mathbf{( 2 5 - 3 0 )}\end{array}$ & $\begin{array}{c}\text { Obesidad } \\
(\mathbf{2 3 0 )}\end{array}$ \\
\hline Participantes & 128 & 1866 & 699 & 467 \\
\hline Embarazos & 0 & 23 & 9 & 12 \\
\hline Tasa embarazos & $0 \%$ & $1,23 \%$ & $1,29 \%$ & $2,57 \%$ \\
\hline IC95\% & 0 a 2,84 & 0,78 a 1,84 & 0,59 a 2,43 & 1,34 a 4,45 \\
\hline
\end{tabular}

El informe insiste en la necesidad de que las mujeres tomen el anticonceptivo de urgencia lo antes posible después de haber mantenido una relación coital sin protección, independientemente de su peso corporal o IMC.

\subsubsection{Eficacia de los dispositivos intrauterinos de cobre como anticoncepción de urgencia}

La revisión de Cleland 2012 recopiló datos de 7.034 inserciones de dispositivos intrauterinos de 42 estudios, tras las que se observaron un total de 10 Calidad embarazos traducidos en una tasa de fallo del 0,14\% (IC95\% 0,08 a 0,25) (50). 
Los embarazos se repartieron entre dos estudios, uno realizado en China que registró 6 embarazos entre 5629 mujeres (tasa de fallo 0,11\% (IC95\% $0,05$ a 0,23$)$ ), y el único ensayo clínico que ha comparado la inserción de un dispositivo intrauterino frente a conducta expectante (48), que mostró una tasa fallo muy alta ( $2 \%$ (IC95\% 0,69 a 5,03)). La tasa de fallo al excluir los resultados de este ensayo clínico se ajustó al 0,09\% (IC95\% 0,04 a 0,19). La revisión no analizó los datos de seguridad de los dispositivos intrauterinos.

Un estudio incluido en la revisión Cochrane de Cheng 2012 (45) comparó la inserción de un DIU de cobre frente a la mifepristona (49). El estudio solamente registró un embarazo entre las mujeres que recibieron mifepristona (RR 1,51; IC95\% 0,06 a 36,67). En este ensayo la tasa de eventos adversos fue mayor entre las mujeres que recibieron mifepristona (RR 16,59; IC95\% 1,01 a 273,52).

\subsubsection{Anticoncepción de urgencia en otros documentos de recomendaciones}

La CEU de la Faculty of Sexual and Reproductive Healthcare (51) realiza las siguientes recomendaciones relacionadas con los efectos adversos y posibles interacciones con el ulipristal y el levonorgestrel:

- El único método para la anticoncepción de urgencia que puede administrarse en mujeres que toman inductores enzimáticos del metabolismo hepático (o que han dejado esta medicación en los últimos 28 días) es el DIU de cobre.

- La mujeres que toman inductores enzimáticos del metabolismo hepático, incluyendo la profilaxis post-exposición al VIH (o que han dejado esta medicación en los último 28 días), y que no desean o no son elegibles para un DIU de cobre, deberían tomar una dosis de $3 \mathrm{mg}$ de levonorgestrel tan pronto como sea posible antes de las 120 horas posteriores a la relación coital sin protección, a pesar que no se conozca el efecto exacto del levonorgestrel después de las 97 horas.

- No debe recomendarse el ulipristal a aquellas mujeres que toman inductores enzimáticos del metabolismo hepático (o que han dejado esta medicación en los últimos 28 días).

- No debe recomendarse el ulipristal a aquellas mujeres que toman fármacos para aumentar el $\mathrm{pH}$ gástrico (antiácidos, anti-H2 e inhibidores de la bomba de protones).

- Se debe recomendar a las mujeres que consulten con un médico si tienen vómitos durante las 2 horas posteriores a haber tomado levonorgestrel o durante las 3 horas después de haber tomado ulipristal. En este caso se puede ofrecer repetir el mismo método o un DIU de cobre. 
- Debe advertirse a las mujeres de posibles alteraciones del ciclo menstrual tras la anticoncepción de urgencia. Si existieran dudas sobre la menstruación en el ciclo en el que se ha tomado la anticoncepción de urgencia, debería realizarse un test de embarazo tres semanas después de haber recibido la dosis de anticonceptivo.

El mismo organismo ha publicado una declaración en la que ofrece directrices para manejar posibles interacciones entre el ulipristal y otros anticonceptivos hormonales (57), ofreciendo las siguientes recomendaciones:

- Teniendo en cuenta que el ulipristal podría reducir la eficacia de la anticoncepción hormonal, se debe recomendar un método de barrera adicional entre la toma de ulipristal y el inicio del siguiente periodo menstrual.

- No se recomienda el uso conjunto de ulipristal y una anticoncepción de urgencia que contenga levonorgestrel.

La guía del European Consortium for Emergency Contraception solamente hace referencia a los tres métodos autorizados en Europa para anticoncepción de urgencia (dispositivos intrauterinos de cobre, y píldoras de acetato de ulipistral $30 \mathrm{mg}$ o levonorgestrel 1,5 mg). El documento destaca la efectividad de estos métodos que pueden reducir el riesgo de embarazo tras una relación coital sin protección o con protección inadecuada entre un $75 \%$ y un $99 \%$ en función del método (52). El documento destaca el DIU de cobre como el método de anticoncepción de urgencia más efectivo seguido de las píldoras de ulipristal y levonorgestrel, aclarando que la efectividad de la anticoncepción de urgencia oral está relacionada con el momento del ciclo en el cual se toma la medicación.

Este documento formula las siguientes recomendaciones sobre los métodos de anticoncepción de urgencia que pueden ofrecerse:

- Es necesario informar a las usuarias sobre las opciones de anticoncepción de urgencia disponibles y de la limitación en su efectividad dependiendo del momento en que se usa;

- En caso de que solamente haya habido una relación coital sin protección las opciones, dependiendo del tiempo transcurrido desde la relación sin protección, son:

i) Menos de 72 horas: DIU de cobre, seguido de la píldora de ulipristal y de la píldora de levonorgestrel;

ii) Entre 72 y 120 horas: DIU de cobre, seguido de la píldora de ulipristal. La píldora de levonorgestrel no tiene indicación en esta situación, pero puede ser efectiva hasta las 96 horas; 
iii)Más de 96 horas: el DIU de cobre puede ser una opción si se cree que la mujer se encuentra antes de la primera fecha del inicio de la implantación (mínimo de seis días tras la relación sin protección y alrededor del día 20 de un ciclo de 28 días).

Si ha habido más de una relación coital sin protección las opciones son:

i) El DIU de cobre es el método más efectivo y puede utilizarse en cualquier momento hasta el día en el que el inicio de la implantación pudiera haber tenido lugar (mínimo de seis días tras la relación sin protección y alrededor del día 20 de un ciclo de 28 días);

ii) La píldora de ulipristal puede utilizarse si todos los episodios de coito sin protección han sucedido dentro de las últimas 120 horas;

iii)La píldora de levonorgestrel se considera segura para su empleo fuera de las restricciones de uso aprobadas.

NICE ha elaborado un estándar de calidad que establece que las mujeres que realizan una consulta de anticoncepción de urgencia deben ser informadas de que el dispositivo intrauterino es más efectivo que los métodos disponibles por vía oral (53). El documento especifica que el DIU de cobre tiene una tasa de fracaso menor que los métodos orales de anticoncepción de emergencia. Por otro lado, una vez insertado, puede ser utilizado como método anticonceptivo de manera continua.

\subsection{Aspectos relacionados con la libre dispensación de la anticoncepción de urgencia}

Se ha identificado una revisión sistemática Cochrane ((58), fecha de búsqueda en noviembre de 2009) que comparó la dispensación por adelantado de la anticoncepción de urgencia sobre las tasas de embarazo, infecciones de transmisión sexual y comportamientos sexuales y uso de anticonceptivos. La revisión sistemática incluyó cuatro ensayos que evaluaron el desenlace de la tasa de infecciones de transmisión sexual (ITS) (59-62).

Dos revisiones sistemáticas (fecha de búsqueda en noviembre de 2009 (63); y fecha de búsqueda febrero de 2012 (64)) evaluaron la misma intervención que la revisión de Polis 2009 (58), incluyendo los mismos estudios y mostrando los mismos resultados que ésta.

Los cuatro ensayos incluidos en las revisiones sistemáticas $(59,60,61,62)$ dispensaron levonorgestrel como anticoncepción de urgencia.

Ekstrand 2008 incluyó 420 adolescentes suecas (entre 15 y 19 años) en una clínica de un hospital universitario (59). El ensayo evaluó la dispensación de una dosis única de $1,5 \mathrm{mg}$ de levonorgestrel y 10 preservativos, juntamente con un folleto informativo, comparado con una dosis estándar de levonorgestrel.

Gold 2004 incluyó a 301 adolescentes norteamericanas (entre 15 y 20 años) principalmente procedentes de familias con bajos ingresos o de minorías (60). El ensayo evaluó la 
dispensación de dos dosis de $0,75 \mathrm{mg}$ de levonorgestrel con la posibilidad de obtener dos dosis extras durante 6 meses a demanda, además de recibir un folleto informativo. Esta intervención se comparó con la libre dispensación de la anticoncepción de urgencia en una clínica.

Raine 2005 incluyó a 1228 adolescentes y jóvenes (15 a 24 años) de habla inglesa o hispana en San Francisco, procedentes de familias con bajos ingresos, que estuvieran dispuestas a participar en el ensayo durante 6 meses (61). El estudio evaluó la dispensación de tres dosis de $0,75 \mathrm{mg}$ de levonorgestrel para ser administradas con un intérvalo de 12 horas, en las 72 primeras horas tras la relación coital sin protección. Las participantes en el grupo control recibieron la libre dispensación de la anticoncepción de urgencia en una clínica.

Raymond 2006 incluyó a 1409 adolescentes y jóvenes (14 a 24 años) que acudieron a varias clínicas de planificación familiar en dos estados norteamericanos (62). Se evaluó la dispensación de dos dosis de $0,75 \mathrm{mg}$ de levonorgestrel con la posibilidad de obtener más dosis en el transcurso del estudio. Las participantes en el grupo control accedieron a la dispensación de anticoncepción de urgencia habitual en las mismas clínicas.

Cabe destacar las limitaciones en el diseño de los ECA de Ekstrand 2008 (59) y Gold 2004 (60) que tuvieron una importante tasa de pérdidas (entre $22 \%$ y $26 \%$, que en el caso de Gold 2004 fueron mucho más considerables entre las participantes del grupo intervención (60)), y además no fueron estudios con el suficiente tamaño muestral como para demostrar diferencias en los principales desenlaces de interés. Del mismo modo, el hecho de que en todos los estudios se dispensara levonorgestrel a todas las participantes, sin disponer de la comparación de la dispensación de levonorgestrel con un control no activo u otro tipo de anticoncepción de urgencia, reduce la confianza en los resultados disponibles en la literatura.

Los estudios incluidos en las revisiones sistemáticas de Polis 2009 (58) y Meyer 2011 (63) mostraron que la libre dispensación por adelantado de leCalidad vonorgestrel como anticoncepción de urgencia no incrementa la incidencia de infecciones de transmisión sexual u otras conductas sexuales que pudieran aumentar el riesgo de embarazo.

Comparada con la dispensación estándar, la libre dispensación de la píldora de levonorgestrel no aumentó la incidencia de infecciones de transmisión sexual (4 ECA, 3.123 mujeres, 6,5\% dispensación estándar frente a 6,3\% libre dispensación; OR 1,01; IC 95\% 0,75 a 1,37). En cuanto al uso de métodos anticonceptivos, las mujeres que tuvieron acceso a la libre dispensación de la anticoncepción de urgencia mostraron una tasa similar de relaciones coitales sin protección que las mujeres que accedieron a la dispensación estándar (5 ECA, 2.024 mujeres; OR 0,96; IC 95\% 0,79 a 1,16). Un análisis de subgrupos del grupo control del estudio de Raymond 2006 (n: 714) mostró que tras 6 meses de seguimiento el $2 \%$ de las mujeres que habían recibido una píldora de levonorgestrel como método de anticoncepción de urgencia presentaron una infección de transmisión sexual (13/714) (62), cifra que aumentó al 6\% a los 12 meses de seguimiento (40/692) (Sander 2009). 
Aunque las mujeres que accedieron a una dispensación anticipada de anticoncepción de urgencia con levonorgestrel tomaron la píldora unas 13 horas antes que las mujeres que accedieron a la dispensación estándar (2 ECA, 1247 mujeres; DM -12,98; IC 95\% -16,66 a -9,31), no se observaron diferencias en la tasa de embarazos (7 ECA, 4217 mujeres; OR 0,82; IC $95 \%$ 0,64 a 1,05).

\section{De la evidencia a la recomendación}

\section{Resumen de la evidencia}

Se dispone de métodos de anticoncepción de urgencia con una alta efectividad que pueden reducir el riesgo de embarazo tras una relación coital sin protección o con protección inadecuada entre un $75 \%$ y un $99 \%$ en función del método $(46,47)$. EL DIU es el método de anticoncepción de urgencia más efectivo seguido de las píldoras de ulipristal y levonorgestrel $(52,53)$.

No se ha demostrado una diferencia relevante en la tasa de embarazos entre el levonorgestrel y el ulipristal al administrarlos como anticonceptivos de urgencia. En el caso del ulipristal se puede administrar en las primeras 120 horas tras la relación coital sin protección, y en el caso del levonorgestrel se ha mostrado su eficacia hasta las 96 horas.

Ambos tratamientos no presentan diferencias importantes en la tasa de efectos adversos, siendo la cefalea en más común en el $20 \%$ de las mujeres. En un ECA, el ulipristal se asoció en mayor medida a la cefalea, náuseas, fatiga, mareos o lumbalgia, mientras que el levonorgestrel lo hizo a la dismenorrea y dolor abdominal. A pesar de que el perfil de seguridad de ambos tratamientos parece similar, todavía se disponen de pocos datos relacionados con el ulipristal. Los datos sobre la pérdida de eficacia del acetato de ulipristal dependiendo del IMC de las usuarias son limitados y poco concluyentes.

Los dispositivos intrauterinos en la anticoncepción de urgencia han mostrado una tasa de fallo que oscila entre el 0,09\% y el 0,14\% (50). Los efectos adversos asociados a la inserción de estos dispositivos en este contexto no se han evaluado correctamente. No se dispone de información de calidad, por otro lado que haya comparado la eficacia de los dispositivos intrauterinos con otros anticonceptivos hormonales.

En mujeres jóvenes la libre dispensación de la píldora de levonorgestrel como anticoncepción de urgencia comparada con la dispensación estándar

Calidad moderada

Calidad moderada

Calidad baja

Calidad baja

Calidad baja no aumenta el riesgo de infecciones de transmisión sexual. 


\section{Calidad de la evidencia}

La calidad global es moderada, baja para los dispositivos intrauterinos como anticoncepción de urgencia.

Se ha bajado la calidad de la evidencia en sólo un grado debido a que los pocos eventos observados en la comparación de ulipristal frente a levonorgestrel llevan a una imprecisión en los estimadores del efecto. Por otro lado la información sobre los efectos adversos es limitada. Los estudios considerados en la revisión de Cleland 2012 sobre dispositivos intrauterinos como anticoncepción de urgencia son en su mayoría observacionales y no controlados, lo que lleva a una baja confianza en los estimadores del efecto que ofrece (50).

La calidad global es baja para la libre dispensación de anticonceptivos de urgencia. Varios de los estudios sufrieron pérdidas en el seguimiento considerables, o no tenían al poder estadístico suficiente para mostrar diferencias significativas entre los grupos. Además las comparaciones en los estudios disponibles dispensaron levonorgestrel a todas las participantes, sin posibilidad de conocer los resultados comparados con un control no activo u otro tipo de anticoncepción de urgencia.

\section{Valores y preferencias de las usuarias}

Ver apartado de Justificación.

\section{Beneficios y riesgos/carga de las intervenciones}

No se ha considerado este aspecto en esta pregunta clínica.

\section{Balance beneficio/riesgo}

EL DIU es el método de anticoncepción de urgencia más efectivo. El ulipristal y el levonorgestrel no han mostrado diferencias importantes en la tasa de efectos adversos. En uno de los ECA disponibles, el ulipristal se asoció en mayor medida a la cefalea, náuseas, fatiga, mareos o lumbalgia, mientras que el levonorgestrel lo hizo a la dismenorrea y dolor abdominal. A pesar de que el perfil de seguridad de ambos tratamientos parece similar, todavía se disponen de pocos datos relacionados con el ulipristal. Por otro lado, los datos reflejados en la ficha técnica del ulipristal sobre la pérdida de eficacia en mujeres con un IMC elevado (56) no son suficientemente concluyentes como para realizar consideraciones especiales en esta circunstancia.

\section{Uso de recursos y costes}

Un estudio de coste eficacia y coste utilidad evaluó el uso del ulipristal en la anticoncepción de urgencia en las 120 primeras horas tras el coito sin protección (65). Se comparó la dosis única de ulipristal $(30 \mathrm{mg}$ ) con la de levonorgestrel, tomando los datos de eficacia del ECA y metanálisis de Glasier 2010 (46). Los autores diseñaron un árbol de decisión para estimar los siguientes desenlaces: embarazo, embarazo seguido de aborto inducido, abor- 
to, embarazo ectópico, y parto vaginal o por cesárea. Se diseñó el estudio con una perspectiva de la sociedad en el contexto de la atención ambulatoria en los EEUU. Los costes se estimaron para los costes de un embarazo y los fármacos comparados expresados como dólares americanos con valor para el año 2011. Se calcularon QALY (Quality Adjusted Life Years: que sintetizan los resultados de salud en un indicador que combina el número de años vividos con la calidad de vida experimentada durante esos años) asumiendo que tras un aborto inducido una mujer viviría 55 años adicionales, con 0,992 QALY por año de vida adicional. El modelo mostró que el uso rutinario de ulipristal en lugar de levonorgestrel resultaría en 37500 embarazos no deseados menos por año, con una ganacia de 8053 QALY y un ahorro de 116 millones de dólares. Los análisis de sensibilidad mostraron que el ulipristal era más coste efectivo que el levonorgestrel en el $95 \%$ de las simulaciones.

\section{Justificación}

No existen grandes diferencias en la tasa de embarazos entre el levonorgestrel y el ulipristal al administrarlos como anticonceptivos de urgencia. Aunque el ulipristal se puede administrar en las primeras 120 horas tras la relación coital sin protección, en el caso del levonorgestrel solamente se dispone de datos sobre su eficacia hasta las 96 horas. Los datos sobre los efectos adversos son insuficientes, sin mostrar diferencias relevantes entre las dos intervenciones.

Los dispositivos intrauterinos son el método más efectivo, con una tasa de fallo entre el $0,09 \%$ y el $0,14 \%$, aunque no se dispone de datos fiables sobre su eficacia comparada a los anticonceptivos hormonales, ni sobre su seguridad. La recomendación sobre el uso del DIU de cobre en anticoncepción de urgencia toma como referencias los criterios de elegibilidad de la OMS (4), en los que se puede considerar su uso hasta 5 días después del coito sin protección o posteriormente cuando se puede estimar la fecha de ovulación, si no han transcurrido más de 5 días desde esta fecha.

Las recomendaciones recogen las consideraciones reflejadas en la ficha técnica de los productos y las diferentes recomendaciones disponibles. Por otra parte, la Estrategia de Salud Sexual y Reproductiva del SNS pone de manifiesto que no existe un protocolo común para el Sistema Nacional de Salud que establezca las condiciones de la prestación de la anticoncepción de urgencia en los servicios de salud, lo que implica fuentes potenciales de inequidad en la accesibilidad (MSPS 2011). En este sentido, es necesario comentar que en España puede accederse a la anticoncepción de urgencia sin receta médica a través de todas las oficinas de farmacia. 


\section{Recomendaciones}

\begin{tabular}{|l|l|}
\hline $\begin{array}{l}\text { Buena } \\
\text { práctica } \\
\text { clínica }\end{array}$ & $\begin{array}{l}\text { Es necesario informar a las mujeres que realizan una consulta de anticon- } \\
\text { cepción de urgencia sobre las opciones de anticoncepción de urgencia dispo- } \\
\text { nibles, de la mejor efectividad del DIU de cobre sobre los métodos orales, y } \\
\text { de la limitación en la efectividad dependiendo del tiempo transcurrido desde } \\
\text { la relación coital sin protección. }\end{array}$ \\
\hline Fuerte & $\begin{array}{l}\text { En aquellas situaciones en las que se decida insertar un DIU de cobre como } \\
\text { anticoncepción de urgencia, debe insertarse en las 120 horas (5 días) de ha- } \\
\text { ber mantenido relaciones coitales sin protección o haberse producido un } \\
\text { fallo del anticonceptivo utilizado. En el caso que pueda estimarse la fecha de } \\
\text { ovulación, el dispositivo puede insertarse más tarde, siempre que no hayan } \\
\text { transcurrido 5 días tras esta fecha. }\end{array}$ \\
\hline Fuerte & $\begin{array}{l}\text { En aquellas situaciones en las que se decida utilizar un método oral como } \\
\text { anticoncepción de urgencia (acetato de ulipistral 30 mg o levonorgestrel } \\
1,5 \text { mg), se recomienda administrar el tratamiento lo antes posible después } \\
\text { de haber mantenido relaciones coitales sin protección o haberse producido } \\
\text { un fallo del anticonceptivo utilizado (antes de las 120 horas (5 días) en el } \\
\text { caso del acetato de ulipristal o de las 72 horas (3 días) en el caso del levon- } \\
\text { orgestrel). }\end{array}$ \\
\hline $\begin{array}{l}\text { Fuerte } \\
\text { práctica } \\
\text { clínica }\end{array}$ & $\begin{array}{l}\text { En aquellas situaciones en las que se decida utilizar un método oral como } \\
\text { anticoncepción de urgencia, se recomienda administrar de nuevo el anti- } \\
\text { conceptivo si se producen vómitos en las tres horas siguientes a la toma del } \\
\text { comprimido. }\end{array}$ \\
\hline $\begin{array}{l}\text { Se sugiere la elaboración de protocolos para la provisión y manejo de la } \\
\text { anticoncepción de urgencia que garantice la accesibilidad a las mujeres que } \\
\text { la necesiten. }\end{array}$ \\
\hline
\end{tabular}





\section{Manejo clínico de los métodos anticonceptivos}

Preguntas a responder:

- ¿Cuáles son los requisitos previos para la prescripción de un anticonceptivo hormonal?

- ¿Cuáles son los controles necesarios para el seguimiento de un anticonceptivo hormonal?

- ¿Cuáles son los requisitos previos para la inserción de un dispositivo intrauterino?

- ¿Cuáles son los controles necesarios para el seguimiento en usuarias de un dispositivo intrauterino?

- ¿Cómo se manejan los sangrados derivados del uso de anticoncepción?

- ¿Cómo se manejan los olvidos de las píldoras combinadas y de solo gestágeno?

- ¿Cuál debe ser el manejo de la anticoncepción hormonal en caso de interacción medicamentosa?

- ¿Se debe utilizar la profilaxis antibiótica en alguna circunstancia en la inserción de un DIU?

- ¿Es útil el uso de misoprostol para facilitar la inserción de un DIU?

\subsection{Consideraciones previas}

\subsubsection{Requisitos previos para la prescripción de un anticonceptivo hormonal y dispositivos intrauterinos}

Una revisión sistemática evaluó los resultados derivados de la exploración física previa a la prescripción de anticonceptivos hormonales (66), focalizando en la exploración mamaria y pélvica. Los mismos autores realizaron una revisión sistemática para determinar si es necesario medir la presión arterial durante la exploración previa a la prescripción de un anticonceptivo hormonal (67), y otra focalizada en la necesidad de realizar pruebas de laboratorio antes de iniciar el tratamiento con un anticonceptivo (68). Se han valorado estas revisiones, así como los estudios más relevantes incluidos en éstas. 


\subsubsection{Exploración física y pruebas de laboratorio}

\subsection{Toma de presión arterial}

Una revisión (67) incluyó los resultados de tres estudios de casos y controles que incluyeron usuarias de anticonceptivos hormonales orales combinados (68-70), y focalizaron el análisis en el riesgo de infarto de miocardio (69 - 71), e ictus isquémico $(69,70)$ o hemorrágico (70). En dos estudios se compararon directamente los resultados de las mujeres a las que se les había tomado la presión arterial con las que no $(70,71)$ y en el otro se contrastaron los datos de las mujeres a las que se había tomado la presión arterial y a las que no, dependiendo de si eran usuarias de anticonceptivos o no (69).

En uno de los estudios el riesgo de infarto de miocardio fue mayor en aquellas mujeres en las que no se había medido la presión arterial antes

Calidad baja de iniciar el uso de un anticonceptivo oral combinado en todos los análisis de subgrupos que se realizaron (OR 9,47; IC95\% 3,72 a 24,1), aunque los intervalos de confianza se solapaban con los estimadores del efecto para las mujeres a las que sí se les había tomado la presión arterial (OR 2,60; IC95\% 1,15 a 5,89) (69).

El riesgo fue mayor en las mujeres a las que no se les había tomado la presión arterial cuando se estratificó por edad, la dosis de estrógeno o el tabaquismo. El mayor impacto se observó en las mujeres europeas a las que no se les tomó la presión arterial y eran fumadoras (OR 71,4; IC95\% 16,5 a 309).

El estudio Transnational Study también mostró un mayor riesgo de infarto de miocardio entre las usuarias de anticonceptivos orales combinados a las que no se había tomado la presión arterial (OR 1,81; IC95\% 1,20 a 2,73) (70). El estudio MICA tuvo resultados similares pero no mostró diferencias estadísticamente significativas entre las mujeres dependiendo de la medida de la presión arterial (OR 2,07; IC95\% 0,81 a 5,30).

En el estudio de WHO también se observó un mayor riesgo de ictus isquémico en aquellas usuarias de anticonceptivos orales combinados sin una toma de presión arterial antes de iniciar el tratamiento (OR 3,90; IC95\% 1,83 a 8,33 ), aunque los intervalos de confianza se solaparon con los del estimador de las mujeres a las que se les había realizado esta exploración (OR 2,26; IC95\% 1,12 a 4,55) (69). Este aumento de riesgo también se observó en el estudio Transnational Study. En el caso del ictus hemorrágico ninguno de los estudios mostró ninguna asociación. 


\subsection{Exploración mamaria y pélvica}

Una revisión sistemática no identificó estudios que valoraran la utilidad de la exploración mamaria antes de la prescripción de un anticonceptivo hormonal (66). Los autores comentan que el resultado de la exploración mamaria en este contexto es incierto dado que la proporción de casos de cáncer de mama que se diagnostican en las mujeres en edad reproductiva es baja. En un estudio que evaluó la prevalencia de patología mamaria en mujeres a las que se les realizaba de manera rutinaria una exploración mamaria en un centro de planificación familiar, mostró que entre más de 13.000 mujeres evaluadas en un periodo de 5 años, solamente se detectó en 12 mujeres un tumor invasivo, de las que ninguna era menor de 35 años y la mayoría era mayor de 40 años (72).

Por otro lado, una revisión sistemática Cochrane ha mostrado que el autoexamen o examen clínico de la mama no aportan ningún beneficio como métodos de cribado para un diagnóstico precoz del cáncer de mama (73). La revisión incluyó un ensayo de base poblacional en el que se combinaba la exploración mamaria con el autoexamen, aunque el estudio se suspendió debido a la falta de cumplimiento con el seguimiento.

Dos estudios de cohorte retrospectivos compararon los resultados de dos programas en los que se prescribía un anticonceptivo oral combinado sin haber realizado una exploración pélvica previa con los resultados de un grupo de mujeres a las que se les había realizado una exploración pélvica $(74,75)$. Se valoró la incidencia de ITS, o la tasa de resultados de una citología vaginal anormales.

Un estudio evaluó los resultados de un programa en EEUU en el que adolescentes (menores de 18 años) en su primera visita de planificación familiar podían retrasar la exploración pélvica durante seis meses, periodo durante el que recibían un método anticonceptivo. De las 390 adolescentes que consultaron en el centro de salud, solamente se recabaron datos de 151 entre las que 40 retrasaron la exploración y recibieron un anticonceptivo, mientras que a 111 se les realizó la exploración. La revisión de las historias clínicas de ambos grupos, no mostró diferencias significativas en la incidencia de gonorrea, clamidia, sífilis, o resultados anormales de una citología vaginal (74).

En el segundo estudio se compararon los resultados de 400 usuarias de anticonceptivos que recibieron la prescripción en un centro no sanitario con las de 400 usuarias emparejadas por edad y raza que habían conseguido la prescripción en un centro sanitario (75). A la gran mayoría de éstas últimas se les había realizado una exploración pélvica como rutina en la consulta de anticoncepción. Los dos grupos comparados no mostraron diferencias en la incidencia de ITS, resultados anormales en una citología vaginal, la edad temprana del primer coito, las múltiples parejas sexuales o el tabaquismo (considerados por los autores factores de riesgo de cáncer de cérvix).

Calidad muy baja 


\subsection{Pruebas de laboratorio}

Una revisión sistemática evalúa la necesidad de varias pruebas de laboratorio antes de iniciar el tratamiento con un anticonceptivo: nivel de glucosa, perfil lipídico, función hepática, citología, y cribado de ITS y VIH (68).

Sobre el nivel de glucosa destaca el posible impacto de algunos anticonceptivos hormonales en la tolerancia a la glucosa y la sensibilidad a la insulina, como el caso del implante o inyectable de gestágeno asociado a un Calidad baja aumento del nivel de insulina y los niveles de glucosa (76) o la disminución del nivel de insulina y aumento de los niveles de glucosa en algunas mujeres diabéticas usuarias del acetato de medroxiprogesterona $(76,77)$. En mujeres con diabetes los anticonceptivos orales combinados han mostrado un aumento de los niveles de glucemia en ayunas, sin repercutir en eventos más graves (77). Una revisión Cochrane ha mostrado que el impacto de los anticonceptivos hormonales en el metabolismo de la glucosa no es clínicamente relevante, a pesar de las limitaciones de los estudios que han evaluado este tema (78).

A pesar de que los criterios de elegibilidad para el uso de anticonceptivos establecen algunas contraindicaciones para algunos métodos en mujeres con determinadas complicaciones vasculares debidas a la diabetes (4), este diagnóstico debería identificarse en una anamnesis y es poco probable que justifique la realización de una prueba para determinar el nivel de glucosa.

Un estudio de cohorte mostró que las usuarias de anticonceptivos orales combinados y de acetato de medroxiprogesterona pueden experimentar un aumento de los niveles de triglicéridos, colesterol o de las lipoproteínas de muy baja densidad, aunque la alteración fue limitada en el tiempo y su impacto clínico no es relevante (79). Por otro lado, una revisión recoge datos que muestran que la prevalencia de mujeres en edad fértil con hipercolesterolemia es baja (68). Como en el caso anterior, a pesar que los criterios de elegibilidad para el uso de anticonceptivos contemplan alguna situación en la que debería evitarse el uso de anticonceptivos hormonales combinados en mujeres con hiperlipidemia (4), el cribado rutinario de esta alteración no parece estar justificado.

Una revisión sistemática evaluó el impacto de los anticonceptivos hormonales en las usuarias con hepatitis o cirrosis hepática (80), e incluyó un estudio que no mostró un efecto de los anticonceptivos orales sobre el curso de la hepatitis aguda y otros cinco estudios que no mostraron un efecto de estos anticonceptivos sobre la hepatitis crónica o la cirrosis hepática o su progresión.

Otra revisión de los mismos investigadores evaluó el impacto de los anticonceptivos hormonales en las usuarias con tumores hepáticos (81). Ninguna de las dos series de casos incluidas en la revisión mostró un impacto de los anticonceptivos orales combinados (o de los anticonceptivos de sólo gestágeno) en las lesiones hepáticas o su progresión. 
Es esperable que las mujeres con una patología hepática aguda o crónica muestren alguna sintomatología o que ésta pueda identificarse en el transcurso de una anamnesis (68), por lo que el estudio rutinario de la función hepática no estaría justificado.

La mayoría de casos de cáncer de cérvix aparecen entre los 35 y 50 años, con una incidencia anual de 34.000 casos y 16.000 muertes en la Unión Europea, aunque es uno de los tumores que mejor se puede controlar con programas de cribado (82). Se ha calculado que siguiendo la recomendación de realizar un cribado a nivel poblacional en un intervalo de entre cada tres y cinco años, la incidencia de cáncer de cérvix podría disminuir hasta en un $80 \%$ (83), sin dejar de lado, sin embargo, los retos para el sistema sanitario que implantar este tipo de programas.

Aunque el uso prolongado de anticonceptivos hormonales es uno de los factores de riesgo de cáncer de cérvix $(83,84)$, y teniendo en cuenta que en el estado español hasta el $75 \%$ de las mujeres de entre 18 y 65 años se han realizado una citología en los últimos 3 años (85), la realización rutinaria de citologías en el momento de la consulta anticonceptiva no estaría indicada (82), aunque puedan ser una oportunidad para valorar la adherencia a los programas de cribado.

En cuanto a los dispositivos intrauterinos es necesario destacar que los criterios de elegibilidad para el uso de anticonceptivos establecen algunas contraindicaciones para las mujeres con cáncer de cérvix (4).

En el caso de las mujeres con alto riesgo o con una ITS establecida y en las mujeres con alto riesgo de VIH o con una infección por VIH, existen contraindicaciones para la inserción de dispositivos intrauterinos (4). En el caso del VIH, aunque existen anticonceptivos indicados para las mujeres con riesgo de VIH o con una infección por VIH, es importante aconsejar el uso del preservativo de manera adicional.

Aunque no existen estudios que hayan comparado el riesgo de enfermedad inflamatoria pélvica (EIP) en mujeres con una ITS dependiendo de si usaban un DIU o no, una revisión sistemática valoró los resultados de seis estudios observacionales en los que compararon los resultados de usuarias de DIU de cobre dependiendo de si tenían una ITS o no en el momento de la inserción (86). Los estudios mostraron que las mujeres con clamidia o gonorrea en el momento de la inserción del DIU tenían un mayor de riesgo de EIP que las mujeres sin infección, a pesar que el riesgo en términos absolutos fue bajo en ambos grupos (entre 0 y 5 de cada 100 en las mujeres con ITS al inicio del estudio frente a 0 a 2 casos de cada 100 en las mujeres sin ITS al inicio del estudio). 
En mujeres con un riesgo bajo de ITS el uso de dispositivos intrauterinos se asocia raramente a la EIP, por lo que sería suficiente que se incorporen en la anamnesis aspectos relacionados con los hábitos sexuales de las futuras usuarias con el objetivo de descartar el riesgo de ITS, sin que sea necesario realizar serologías de manera rutinaria. En caso de detectar signos o síntomas de alguna ITS, es necesario realizar una serología y esperar su resultado, y no insertar un DIU hasta que se complete el tratamiento en caso de un resultado positivo (157).

El documento de la OMS "Selected practice recomendations for contraceptive use" (88) y adaptaciones posteriores (3) realizan una propuesta sencilla sobre las exploraciones y pruebas necesarias antes de la prescripción de anticonceptivos. Estas propuestas no recomiendan la realización rutinaria de exploraciones o pruebas de laboratorio, con alguna excepción como la toma de presión arterial antes de prescribir un anticonceptivo hormonal, o el examen pélvico antes de la inserción de un DIU. El Algoritmo 1 recoge esta propuesta de exploraciones necesarias para la prescripción de anticonceptivos.

\subsubsection{Anamnesis y contenido de la visita previa a la prescripción de un anticonceptivo}

Los requisitos previos a la prescripción de un método anticonceptivo se centran en la identificación de factores de riesgo y a los antecedentes patológicos que contraindicarían su uso, que permitirían aplicar los diferentes criterios de elegibilidad disponibles (4).

Por este motivo en el momento previo a la elección de un método anticonceptivo se debería realizar una anamnesis orientada a detectar factores de riesgo y antecedentes patológicos, que según algunas directrices debería repetirse anualmente (89). Debe hacerse hincapié en los antecedentes personales o familiares, la toma de medicamentos u otros productos como remedios naturales, y la presencia de migraña o factores de riesgo cardiovascular (8).

También se debería facilitar la información adecuada sobre normas de uso, efectos secundarios, riesgos y beneficios de los diferentes métodos (8). Por último, cabe la posibilidad de aprovechar la visita de la usuaria para realizar actividades preventivas y de promoción de la salud (informar sobre el cribado de cáncer de cérvix y de mama, consejo antitabaco, o promoción de hábitos saludables) (8).

En la valoración para la prescripción de un anticonceptivo hormonal combinado (píldoras, parches y anillo vaginal) debería valorarse: la edad, las características de la menstruación, la lactancia materna, el posparto (incluido el parto pre-término), la cefalea y migraña, los antecedentes de diabetes, hipertensión, tabaquismo, obesidad, hiperlipidemias, enfermedad cardiaca isquémica o accidente cerebrovascular, trombosis venosa, neoplasia de mama, enfermedad autoinmune, enfermedad hepática, enfermedad de la vesícula biliar, trombofilias, infecciones de transmisión sexual, tuberculosis, enfermedad cardiaca congénita y las valvulopatías (8).

En el caso de los anticonceptivos hormonales de sólo progestágenos debe evaluarse: la edad, el sangrado menstrual, la lactancia materna, antecedentes de diabetes, hiperten- 
sión, trombosis venosa, enfermedad cardiaca isquémica o accidente cerebrovascular, hiperlipidemias, obesidad, neoplasia de mama, enfermedad de la vesícula biliar y colestasis, enfermedad hepática, enfermedad autoinmune, infecciones de transmisión sexual (8).

Cuando se valore la inserción de un dispositivo liberador de levonorgestrel, conviene identificar a las mujeres con alto riesgo de ITS, y valorar la edad, el embarazo, posparto, post-aborto, antecedente de embarazo ectópico, sangrado abundante, enfermedad trofoblástica gestacional, cáncer de cérvix y endometrio, cavidad uterina distorsionada, enfermedad pélvica inflamatoria, infecciones de transmisión sexual y tuberculosis pélvica (8).

\section{De la evidencia a la recomendación}

\section{Resumen de la evidencia}

Se ha observado un aumento del riesgo de infarto de miocardio y de ictus isquémico en las mujeres a las que, antes de iniciar un tratamiento con anticonceptivos orales combinados, no se realiza una comprobación de su presión arterial en comparación con las mujeres a las que sí se les realizaba esta exploración (67).

La realización de una exploración mamaria o pélvica no aporta ningún valor a la valoración de una mujer que desea iniciar un tratamiento con anticonceptivos (66).

A pesar de que determinados métodos de anticonceptivos hormonales pueden producir alguna alteración en mujeres con diabetes, hiperlipidemia o problemas hepáticos, el cribado rutinario de estos problemas de salud

Calidad baja

Calidad muy baja

Calidad baja aporta poco valor antes del inicio de la prescripción de un anticonceptivo, siendo más adecuado la exploración de estos aspectos en la anamnesis (68).

Existen situaciones en las que se contraindica el uso de un DIU en mujeres con cáncer de cérvix, o con una alta sospecha de infección de transmisión sexual, o que tienen una ITS establecida o una infección por VIH (en un estadio avanzado en el momento de la inserción) (4).

\section{Calidad de la evidencia}

La calidad global es baja.

Los estudios que han valorado el valor de la exploración pélvica fueron retrospectivos y no controlados. Además mostraron graves limitaciones en su diseño (como la medida no controlada del desenlace de interés (75)) o ejecución (sesgo de desgaste (74)), por lo que la calidad de la evidencia se ha considerado muy baja. Para la medición de lípidos y la función hepática se ha bajado la calidad de la evidencia por disponer de estudios observacionales no controlados con una muestra limitada. 
La calidad de la evidencia para los estudios sobre el impacto de medir o no la presión arterial antes de iniciar el tratamiento anticonceptivo es baja, por basarse en estudios de casos y controles con resultados que muestran una cierta imprevisión en sus estimadores (69).

La calidad de la evidencia para el impacto de los anticonceptivos sobre el metabolismo de la glucosa es baja, debido a la amplia variabilidad entre los estudios disponibles, la limitada muestra de muchos de ellos y las limitaciones en el reporte de sus resultados (78).

En ninguno de los casos se ha podido considerar aumentar la calidad de la evidencia; en este caso no se presentan tablas de evidencia GRADE.

\section{Valores y preferencias de las usuarias}

No se ha considerado este aspecto en esta pregunta clínica.

\section{Beneficios y riesgos/carga de las intervenciones}

Las recomendaciones en este apartado se dirigen a la identificación de factores de riesgo que determinen la contraindicación de algún método anticonceptivo, intentando evitar pruebas de poco valor que retrasen la prescripción de métodos eficaces y seguros.

\section{Balance beneficio/riesgo}

La toma de presión arterial ha mostrado consistentemente tener un impacto en las usuarias de anticonceptivos hormonales combinados. Aunque la medida del IMC no es imprescindible en la decisión de cuál puede ser el anticonceptivo más adecuado (reconociendo que puede contraindicar en alguna ocasión), su medida y monitorización puede servir para futuras referencias.

\section{Uso de recursos y costes}

No se ha considerado este aspecto en esta pregunta clínica.

\section{Justificación}

Ver apartados de balance entre beneficio, riesgos, y cargas de las intervenciones. 


\section{Recomendaciones}

\begin{tabular}{|l|l|}
\hline $\begin{array}{l}\text { Buena } \\
\text { práctica } \\
\text { clínica }\end{array}$ & $\begin{array}{l}\text { Antes de prescribir un determinado anticonceptivo se sugiere realizar una } \\
\text { historia clínica que incluya una anamnesis detallada para identificar a mu- } \\
\text { jeres con factores de riesgo y antecedentes patológicos que contraindiquen } \\
\text { su uso, de acuerdo con los diferentes criterios de elegibilidad disponibles } \\
\text { (consultar Algoritmo 1). }\end{array}$ \\
\hline Fuerte & $\begin{array}{l}\text { Antes de prescribir un anticonceptivo hormonal combinado o un inyectable } \\
\text { de solo gestágenos se recomienda tomar la presión arterial y calcular el índi- } \\
\text { ce de masa corporal (consultar Algoritmo 1). }\end{array}$ \\
\hline Débil & $\begin{array}{l}\text { Se sugiere no realizar de manera rutinaria una exploración ginecológica ni } \\
\text { mamaria, o una citología, antes de prescribir un anticonceptivo (consultar } \\
\text { Algoritmo 1). }\end{array}$ \\
\hline
\end{tabular}

\subsection{Controles necesarios para el seguimiento de usuarias de anticonceptivos hormonales e intrauterinos}

\subsubsection{Controles necesarios para el seguimiento en usuarias de anticonceptivos hormonales y DIU}

Una revisión sistemática de estudios controlados ((90); fecha de búsqueda mayo 2012) valoró si las visitas de seguimiento tras el inicio de un método anticonceptivo mejora su uso correcto y su continuidad de uso. La revisión sistemática identificó cuatro estudios sobre el impacto en la continuidad y el uso correcto de los anticonceptivos de controles programados o controles telefónicos. Dos de los estudios valoraron el efecto de visitas programadas tras la inserción de un DIU en la continuación con el método, valorado en términos de extracciones o expulsiones del dispositivo. En una cohorte prospectiva se compararon los resultados de 280 mujeres que tras la inserción de un DIU, seguían dos frecuencias de visitas diferentes, tras un cambio organizativo en el centro sanitario en el que fueron atendidas las usuarias: 199 tuvieron un control a las 6 semanas de la inserción, a los 3, 6 y doce meses, y posteriormente anualmente, mientras que el resto de 81 mujeres tuvieron un control a los 6 meses tras la inserción y posteriormente anualmente (91). Mientras que en la primera parte del estudio el porcentaje de DIU de cobre fue mayor que el de levonorgestrel (75\%), en la segunda parte la mayoría fueron usuarias del DIU de levonorgestrel (63\%). La revisión también incluye los resultados de un ensayo clínico controlado (con asignación por alternancia) que evaluó el impacto del momento en el que se realizaba la primera visita de seguimiento en la continuidad de uso de un DIU. Se asignó a las mujeres a una visita una semana tras la inserción del DIU (n: 175), dos semanas después (n: 126), o un mes después (n: 190) (92).

Otros dos ensayos clínicos valoraron una intervención que incluía un programa de controles telefónicos para mejorar el uso de una serie de anticonceptivos en adolescentes. 
En un ensayo clínico se valoró un programa de controles telefónicos en el uso continuado de anticonceptivos en un grupo de adolescentes (entre 12 y 17 años) que asistieron a una clínica de planificación familiar (93). En este estudio norteamericano, 83 adolescentes recibieron un apoyo periódico descrito como el seguimiento mediante dos a seis llamadas telefónicas en las cuatro a seis semanas posteriores a la primera visita en la clínica (con un seguimiento a los seis y 15 meses). Sus resultados se compararon con los de 201 adolescentes que no recibieron el apoyo pero que tuvieron una entrevista de seguimiento a los seis y 15 meses, y un último grupo de 61 chicas que no recibieron el apoyo y solamente fueron entrevistadas a los 15 meses. La mayoría de participantes usaron los anticonceptivos orales $(82 \%)$, seguidos de los preservativos $(8 \%)$, diafragma $(5 \%)$ y DIU $(1 \%)$. Otro ensayo clínico evaluó un programa de seguimiento mediante llamadas telefónicas de 18 meses de duración en 805 adolescentes norteamericanas que visitaban una clínica de planificación familiar (94). Una persona con experiencia en planificación familiar realizaba una llamada mensual en los primeros seis meses a las participantes del grupo intervención, y posteriormente una llamada cada dos meses en los seis últimos meses del estudio. Las participantes en el grupo control no recibían ninguna llamada y simplemente completaban las visitas de seguimiento. Aunque el $75 \%$ de participantes completaron las visitas de seguimiento solamente se completaron las nueve llamadas de seguimiento en el $30 \%$ de las participantes, y solamente el $11 \%$ recibió seis llamadas o más.

Un estudio de cohorte mostró que, durante el primer año de uso, se extrajeron un menor número de DIU en las mujeres que tuvieron una mayor frecuencia de visitas, que en las que tuvieron una frecuencia menor, sin que la diferencia fuera estadísticamente significativa (14\% frente a $24 \%$; RR 0,6; IC95\% 0,3 a 1,0) (91). Entre aquellas mujeres a las que se les extrajo el DIU por razones médicas en los primeros 12 meses de uso, se observó que el tiempo hasta la extracción era mayor en las mujeres con una mayor frecuencia de visitas independientemente del tipo de DIU usado (6,9 meses frente a 2,1 meses). Dos de las mujeres que recibieron menos controles tuvieron dos expulsiones del DIU inadvertidas (tasa de expulsión inadvertida del 0,27 por 100 mujeres año; IC 95\% 0,02 a 0,8), y las mujeres con más controles programados realizaron una proporción mayor de visitas no programadas (RR 1,6; IC95\% 1,03 a 2,4).

En el ensayo clínico que evaluó la frecuencia de visitas tras la inserción de un DIU (92) no presentó datos de significación estadística entre los grupos de comparación. Al final del seguimiento del estudio la continuación con el uso del dispositivo fue alta entre los grupos (visita a la primera semana tras la inserción $73,8 \%$; visita tras dos semanas $75,9 \%$; visita tras un mes $83,5 \%)$. El cumplimiento de las participantes con las visitas de seguimiento no fue bueno dado que la mitad de las mujeres no asistían a las visitas programadas en la fecha correcta (visita a la primera semana tras la inserción $50 \%$; visita tras dos semanas $40 \%$; visita tras un mes $46 \%$ ), y en un alto porcentaje el seguimiento debía realizarse en casa ya que las mujeres no asistían a la clínica (visita a la primera semana tras la inserción 5\%; visita tras dos semanas $31 \%$; visita tras un mes $69 \%$ ). 
En el ensayo de los controles telefónicos a adolescentes (93) el porcentaje Calidad baja de participantes que utilizaron los contraceptivos de manera ininterrumpida fue moderado (40,7\% en el grupo de controles telefónicos frente a $47,6 \%$ en las que no recibieron el control). El porcentaje de embarazo fue bajo entre las participantes que recibieron al menos una llamada durante su participación en el estudio (65) en comparación con las chicas que pese a ser aleatorizadas al grupo de control telefónico, no recibieron al menos una llamada $(29 \%)$. Este último porcentaje fue mayor incluso que en las chicas que habían sido aleatorizadas al grupo que no recibió ninguna intervención $(14 \%)$. Los autores del estudio no presentaron pruebas estadísticas para interpretar esta diferencia entre los grupos, aunque comentaron que las diferencias no fueron significativas.

El segundo ensayo clínico que evaluó un programa de controles telefónicos a adolescentes (94) no mostró diferencias entre los grupos en el porcentaje de adolescentes que habían usado un método anticonceptivo durante la última relación sexual. En el caso de las usuarias de anticonceptivos hormonales el porcentaje de uso durante la última relación sexual fue el mismo a los 6 (44\%), 12 (43\%) y 18 meses de seguimiento (43\%). Aunque los autores describieron que el $25 \%$ del total de participantes en el ensayo se quedaron embarazadas, no se ofrecieron datos desagregados para comparar el porcentaje en cada uno de los grupos de interés.

Una revisión sistemática incluyó estudios que valoraban la probabilidad de que una mujer sana pudiera sufrir un problema de salud o experimentar un efecto adverso tras iniciar un método anticonceptivo hormonal o intrauterino ((95), fecha de búsqueda abril de 2012). Los autores incluyeron estudios sobre la incidencia de hipertensión, o un cambio significativo en la presión arterial tras el inicio de un anticonceptivo hormonal combinado, la incidencia de enfermedad inflamatoria pélvica tras la inserción de un DIU de cobre o levonorgestrel, o el posible cambio en el peso tras el inicio del inyectable de acetato de medroxiprogesterona.

Una revisión identificó cinco estudios sobre el impacto de los anticoncep- Calidad baja tivos orales en la hipertensión (un ensayo clínico y cuatro cohortes), y no encontraron estudios sobre el impacto del parche o el anillo (95). Uno de los estudios de cohortes (96) no se ha considerado en el desarrollo de esta pregunta clínica por su limitado tamaño de muestra. 
El ensayo clínico aleatorizó 704 mujeres a un anticonceptivo oral $(100 \mu \mathrm{g}$

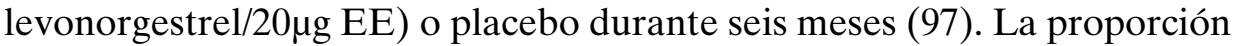
de mujeres con hipertensión al final del estudio fue similar, con siete mujeres en el grupo de anticonceptivos y 8 en el grupo de placebo con una presión arterial $>140 \mathrm{mmHg}$, y seis en el grupo de anticonceptivos frente a 8 en el grupo de placebo con una presión $>90 \mathrm{mmHg}$. Aunque los autores no describieron ninguna prueba estadística para comprobar si las diferencias entre los grupos fueron significativas, el análisis de los resultados con el software de distribución libre RevMan v 5,3 (98) no mostró diferencias entre los grupos (RR >160 mmHg 0,89; IC95\% 0,33 a 2,43; RR >90 mmHg 0,76; IC95\% 0,27 a 2,18).

En un estudio de cohortes prospectivo se valoró el impacto de los anticonceptivos orales en la presión arterial de 485 mujeres con una presión arterial al inicio del estudio inferior a 140/90 mmHg (99). Tras un año de seguimiento la presión arterial aumentó significativamente en las usuarias de anticonceptivos orales. Las 137 usuarias de un anticonceptivo oral con levonorgestrel experimentaron un aumento de $6,4 \mathrm{mmHg}$ en la presión sistólica y $2,7 \mathrm{mmHg}$ en la diastólica. Este aumento significativo también se observó en las 91 mujeres que tomaban un anticonceptivo con diacetato de etinodiol (aumento de 6,2 $\mathrm{mmHg}$ sistólica y 3,0 $\mathrm{mmHg}$ diastólica). Las 143 usuarias de DIU solamente experimentaron una reducción significativa de la presión diastólica de 5,3 $\mathrm{mmHg}$, sin cambios en la sistólica. Las usuarias de anticonceptivos de sólo gestágeno no mostraron un cambio significativo.

Un estudio de cohortes posterior no mostró cambios en la presión arterial estadísticamente significativos durante un año de seguimiento entre 98 usuarias de un DIU y dos grupos de 181 usuarias de dos preparados hormona-

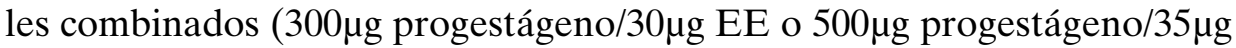
EE) (100).

Un estudio de cohortes prospectivo en 500 mujeres chinas mostró un mayor incremento hasta las 48 semanas, ajustado al nivel de presión arterial al inicio del estudio, en la presión arterial diastólica en las usuarias de anticonceptivos orales comparado con las usuarias de DIU (101). Las diferencias en las mediciones entre ambas usuarias oscilaron entre los $1,8 \mathrm{mmHg}$ a las 12 semanas y los $2,3 \mathrm{mmHg}$ a las 36 semanas. Los cambios en la presión sistólica no mostraron diferencias significativas entre las usuarias de anticonceptivos orales y DIU. En las usuarias del implante no se detectaron cambios relevantes.

Una revisión sistemática identificó cuatro estudios que valoraron la incidencia de enfermedad inflamatoria pélvica (EIP) hasta los 8 años tras la inserción de un DIU de cobre, comparada con la de otros anticonceptivos (95). Para esta pregunta clínica solamente se han valorado los ensayos clínicos incluidos en la revisión $(102,103,104)$. Ninguno de los estudios valorados en la revisión valoró este desenlace en los DIU de levonorgestrel en comparación a otro tipo de anticonceptivo. 
Un estudio realizó un análisis combinado de 12 ensayos clínicos que ofrecían datos sobre la incidencia de EIP hasta los 8 años de seguimiento (102) en 22.900 usuarias de DIU de cobre, liberadores de hormonas (ALZA T IPCS) o anticonceptivos orales. La tasa acumulada de EIP no fue diferente entre los dos tipos de DIU comparados (1,54 EIP por 1000 usuarias/año en los DIU Cu frente a 1,90 EIP por 1000 usuarias/año en los DIU liberadores de hormonas). No hubo diferencias significativas en el riesgo de EIP entre ambos tipos de DIU (RR 0,78; IC95\% 0,38 a 1,64).

Un ensayo clínico comparó la tasa de EIP en 1509 mujeres que fueron aleatorizadas a usar un DIU de levonorgestrel (DIU LNG 20; 755 mujeres) o un DIU de cobre (Copper T 380Ag; 754 mujeres) $(103,105,106)$.

El estudio no identificó una diferencia significativa en la tasa acumulada de expulsiones del DIU debido a EIP. A los 12 meses de seguimiento la tasa acumulada de expulsiones en el grupo del DIU LNG fue de 1,6 por 100 usuarias/año frente a 1,3 por 100 usuarias/año en el grupo de DIU Cu (103). A los 25 meses las tasas acumuladas de expulsión por EIP fue similar (1,3 por 100 usuarias/año en DIU LNG frente a 1,5 en DIU Cu), resultado que se observó durante los siete años de seguimiento del estudio $(105,106)$.

Otro ensayo clínico comparó la incidencia de EIP en 185 mujeres aleatorizadas a un DIU de cobre (Copper T 380Ag) y en 183 aleatorizadas a acetato de medroxiprogesterona (DMPA) (104). No se observaron diferencias en la incidencia de EIP entre ambos métodos anticonceptivos, independientemente de los criterios diagnósticos utilizados ( $\mathrm{RR}$ con criterios $\mathrm{CDC}$ 1,06; IC95\% 0,53 a 2,13; RR con criterios Hagar 1,24; IC95\% 0,34 a 4,53).

Finalmente, la revisión sistemática de Steenland 2013 (95) incluyó dos estudios observacionales sobre el cambio en el peso tras el inicio del inyectable de DMPA.

Un primer estudio en una cohorte de usuarias de DMPA valoró si una ganancia de peso relevante en los primeros meses de uso del anticonceptivo Calidad ( $>5 \%$ sobre el peso al inicio del estudio) podía ser predictiva de una gananbaja cia excesiva de peso con el uso continuado del método (107). El estudio comparó la progresión en la ganancia de peso entre 51 mujeres que ganaron $>5 \%$ del peso inicial en los seis primeros meses de uso del anticonceptivo frente a 144 mujeres que durante estos primeros meses habían ganado $\leq 5 \%$ del peso inicial. 
La ganancia media de peso fue significativamente superior en las mujeres con una ganancia inicial mayor a los $12(8,04 \mathrm{~kg}$ frente a $0,63 \mathrm{~kg}), 24(10,86$ $\mathrm{kg}$ frente a $1,48 \mathrm{~kg})$, y 36 meses de seguimiento (11,08 kg frente a 2,49 kg) $(\mathrm{P}<0,001)$. A los 36 meses de seguimiento las mujeres con una ganancia inicial de peso relevante ganaron alrededor de $7 \mathrm{~kg}$ más que las mujeres con una ganancia de peso más progresiva. Esta ganancia inicial de peso, frente a la ganancia más moderada del grupo control, fue uno de los principales factores predictores de una ganancia de peso relevante a los 36 meses de uso del DMPA.

El segundo estudio de cohortes se diseñó con el mismo objetivo de comprobar si una ganancia de peso relevante inicial podía predecir una ganancia excesiva de peso con el uso excesivo del método anticonceptivo en 97 adolescentes norteamericanas (108).

Las 77 usuarias que tuvieron una ganancia de peso moderado en los seis primeros meses de uso del DMPA apenas variaron su IMC (media de 23,4 a 23,3), mientras que en el otro grupo el aumento en el IMC fue considerable (media de 24,5 a 26,6). A partir de ese momento el incremento de IMC fue significativamente superior en las adolescentes con un aumento de peso inicial considerable que en las que experimentaron un aumento más moderado a los 12 (media de 28,7 frente a 24,2) y los 18 meses de uso (media de $32,1$ frente a 25,7$)$.

\section{De la evidencia a la recomendación}

\section{Resumen de la evidencia}

Los datos sobre el impacto de las visitas de seguimiento en el uso correcto y continuado de los anticonceptivos hormonales y DIU son limitados (90). Un estudio observacional no mostró diferencias significativas en el número de extracciones de un DIU dependiendo de la frecuencia de las visitas de seguimiento, aunque el tiempo hasta la extracción fue mayor a mayor número de visitas realizadas (91). Por otro lado, los ensayos que han evaluado un seguimiento telefónico en usuarias adolescentes de anticonceptivos, no han mostrado mejores resultados de uso que en las usuarias que no recibían el seguimiento $(93,94)$.

Los datos sobre el impacto sobre la presión arterial tras el inicio de un anticonceptivo hormonal oral son inconsistentes (90). En un ensayo clínico se diagnosticó hipertensión en alrededor del $2 \%$ de usuarias de un anticonceptivo oral, sin que la proporción fuera estadísticamente superior a la de las mujeres que tomaron placebo (97). Dos estudios observacionales mostraron un incremento significativo de la presión arterial, en usuarias de anticonceptivos orales $(99,101)$, mientras que otro estudio no mostró estos resultados (100). Los DIU no han mostrado una alteración de la presión arterial relevante en los estudios valorados. 
No se han observado diferencias relevantes en la incidencia de EIP entre las usuarias de diferentes tipos de DIU (de cobre o liberador de hormonas),

Calidad moderada ni al compararla con las usuarias de DMPA o anticonceptivos orales (95).

Dos estudios observacionales han mostrado que las usuarias de DMPA que tienen una ganancia rápida de peso en los primeros seis meses de uso ( $>5 \%$ del peso antes de iniciar el tratamiento), experimentan posteriormente una ganancia de peso e IMC mayor que las usuarias que en los primeros meses experimentan una ganancia de peso moderada $(107,108)$.
Calidad baja

\section{Calidad de la evidencia}

La calidad global es baja.

Globalmente los datos de los diferentes estudios recopilados para el desarrollo de esta pregunta clínica permiten realizar asunciones indirectas para la formulación de las recomendaciones. En ningún caso se han identificado estudios controlados que evalúen de manera directa el número de controles más adecuados para los diferentes métodos anticonceptivos, o cuáles son las pruebas que aportan un mayor valor a estos controles.

En el caso de los estudios que han evaluado la frecuencia de visitas o intervenciones específicas de seguimiento sobre el uso de anticonceptivos, la calidad de la evidencia se considera baja por las limitaciones en el diseño de los estudios y la falta de aplicabilidad de sus resultados. No se han identificado estudios que evalúen programas específicos, comparando por ejemplo diferentes frecuencias de visitas. Los estudios que han evaluado intervenciones en usuarias de DIU fueron un estudio observacional (91) y un ensayo clínico que asignó a las participantes por alternancia y que no comparó los resultados estadísticamente (92). Los ensayos clínicos sobre seguimiento telefónico no presentaron datos desagregados que permitieran valorar el impacto de la intervención en las usuarias de método concreto (93), o implantaron la intervención de manera deficiente, completando el protocolo de estudio solamente en el $30 \%$ de las partipantes (94).

En el caso de los estudios sobre los posibles efectos indeseables tras el inicio de un método anticonceptivo, la calidad de la evidencia se considera moderada para el riesgo de EIP y baja para el riesgo de un aumento de la presión arterial y el peso. Los métodos anticonceptivos evaluados en los estudios sobre la incidencia de EIP en usuarias de DIU pueden haber quedado obsoletos por lo que la calidad de la evidencia estaría afectada por la naturaleza indirecta de los resultados de estos estudios. Por lo que respecta a los estudios que han aportado datos para el aumento de la presión arterial o la ganancia de peso relacionada con el uso de DMPA, en su mayoría son estudios observacionales que o bien no son controlados, o no ofrecen garantías de haber controlado adecuadamente los principales factores de confusión.

\section{Valores y preferencias de las usuarias}

Las participantes en el estudio CHOICE identificaron los atributos más importantes para la elección de un determinado anticonceptivo (109). Éstos fueron la eficacia, la seguri- 
dad, la asequibilidad del método, su duración, y la posibilidad de olvidar el método. En este sentido, una revisión sistemática sobre los aspectos más comunes incluidos en las herramientas de decisión compartida en anticoncepción y en los estudios que evalúan las preferencias de las usuarias de anticonceptivos, mostró que los aspectos relacionados con la seguridad eran los más destacados (37). Estos aspectos pueden ser relevantes para explorar la satisfacción con el uso de un determinado método en las visitas de control.

\section{Beneficios y riesgos/carga de las intervenciones}

La revisión sistemática sobre el número de visitas y su impacto en el uso y continuidad de los anticonceptivos no identificó estudios aplicables de manera directa a la pregunta clínica de interés. De manera indirecta un estudio observacional mostró que una mayor frecuencia de visitas no tenía un impacto en el número de extracciones de DIU, aunque el tiempo hasta la extracción era mayor en comparación con una frecuencia menor (91). Un ensayo clínico con alto riesgo de sesgo no mostró mejores resultados dependiendo del momento en el que se realizaba la primera visita tras la inserción de un DIU (92). Los ensayos que han evaluado programas de seguimiento tampoco han mostrado un beneficio relevante en el uso de los anticonceptivos $(93,94)$.

A pesar de la ausencia de estudios que permitan determinar la frecuencia más adecuada para los controles en la anticoncepción, existen múltiples documentos que establecen por consenso la periodicidad de las visitas de control. En nuestro entorno, la Sociedad Española de Contracepción estableció un consenso en este sentido (8). El documento se adhiere a las recomendaciones recogidas en los criterios de elegibilidad de la OMS que establece que en mujeres sin factores de riesgo se debe realizar una primera visita a los tres meses tras el inicio de un tratamiento hormonal combinado (oral, anillo o parche) o de sólo gestágeno, o a las 3-6 semanas de la inserción en el caso del DIU-LNG. Tras la primera visita recomienda controles anuales en las usuarias de anticonceptivos combinados, y siempre que sea necesario a solicitud de la mujer. El documento también identificó motivos para orientar la retirada del método. En el caso de un anticonceptivo hormonal combinado se identifican la edad (50 años), características del sangrado menstrual, hábito tabáquico, IMC >35, aparición de cefalea o migraña, complicaciones asociadas a la diabetes, hipertensión arterial, enfermedad cardiaca isquémica o accidente cerebrovascular, trombosis venosa, diagnóstico de cáncer de mama, lupus eritematoso sistémico, enfermedad hepática o de la vesícula biliar, VIH o sida, tuberculosis, valvulopatia complicada, cirugía mayor con inmovilización prolongada. Además es necesario revisar si la usuaria está recibiendo un tratamiento con fármacos inductores enzimaticos. En el caso de los anticonceptivos de sólo gestágeno se identifican la certeza de menopausia, características del sangrado menstrual, diagnostico de diabetes, hipertensión, trombosis venosa profunda, enfermedad cardiaca isquémica o accidente cerebrovascular, cáncer de mama, enfermedad hepática, enfermedad autoinmune o infecciones de transmisión sexual. Para orientar la retirada de un dispositivo intrauterino liberador de levonorgestrel, además de los aspectos anteriores se deben evaluar síntomas sugestivos de embarazo, alteraciones en el patrón de sangrado, enfermedad trofoblástica gestacional, cáncer de cérvix y endometrio, enfermedad pélvica inflamatoria, infecciones de trasmisión sexual o tuberculosis pélvica. 


\section{Balance beneficio/riesgo}

Los estudios recogidos en la revisión sobre los efectos indeseados que pueden aparecer tras el inicio de uso de un método anticonceptivo son poco concluyentes. Un ensayo mostró una tasa de diagnósticos de hipertensión de solo el $2 \%$ en usuarias de anticonceptivos orales, sin que la diferencia fuera significativa al compararla con placebo (97). Por otro lado, los estudios que han mostrado un aumento significativo en la presión arterial han mostrado valores al final del estudio que están por debajo de los niveles de hipertensión. Las diferencias en la toma de la presión en los estudios, alejada en ocasiones a la práctica actual, pueden afectar la confianza en sus resultados. Por otro lado los estudios no han mostrado una mayor incidencia de EIP entre los diferentes tipos de DIU, ni en comparación a otros tipos de anticonceptivo. Aunque las diferencias en el diagnóstico de la EIP puede restar fiabilidad a los resultados, el estudio que combinó los resultados de 12 ensayos mostró que la incidencia acumulada de EIP es mayor en los 20 días posteriores a la inserción de un DIU (9,6 por 1000 usuarias/año) que a largo plazo (1,38 por 1000 usuarias/año a los 8 años) (102). Dos estudios observacionales han mostrado resultados consistentes que sugieren que una ganancia de $>5 \%$ del peso inicial en los seis primeros meses de uso del DMPA repercute posteriormente en una ganancia de peso continuada $(107,108)$.

\section{Uso de recursos y costes}

No se han identificado evaluaciones económicas sobre la frecuencia de los controles de los anticonceptivos hormonales o intrauterinos.

\section{Justificación}

La información disponible en la literatura científica no permite formular una recomendación sobre cómo establecer la frecuencia de las visitas para el control de los anticonceptivos hormonales e intrauterinos, pero tampoco existe ningún dato que permita asegurar que deban realizarse visitas de rutina.

Los resultados de la literatura aportan datos, aunque limitados, que permiten identificar algunos aspectos relacionados con los efectos indeseados o adversos derivados del uso de los métodos anticonceptivos. Los cambios en la presión arterial y el peso, en las usuarias de DMPA, parecen ser los dos aspectos que pueden tener un cierto impacto al inicio del uso de un anticonceptivo. Por otro lado, los estudios sobre los atributos identificados por las usuarias como los más relevantes para elegir un anticonceptivo, muestran que la seguridad del método y los posibles efectos indeseados son alguno de los aspectos más importantes. Por este motivo, de manera similar a otras guías de práctica clínica, las recomendaciones formuladas en este apartado se dirigen a identificar aquellos aspectos más relevantes derivados del uso de un determinado método anticonceptivo. Se ha graduado la fuerza de aquéllas recomendaciones en las que se dispone de datos en la literatura. 


\section{Recomendaciones}

\begin{tabular}{|l|l|}
\hline $\begin{array}{l}\text { Buena } \\
\text { práctica } \\
\text { clínica }\end{array}$ & $\begin{array}{l}\text { En el momento en que la mujer escoja un determinado método anticoncep- } \\
\text { tivo, se sugiere informarla sobre la conveniencia de consultar cualquier efec- } \\
\text { to secundario u otros problemas derivados del método anticonceptivo o si } \\
\text { desean cambiar el método. Las usuarias también deben recibir información } \\
\text { sobre los principales síntomas de alerta que permitan identificar situaciones } \\
\text { en las que deba considerarse una modificación en el uso del anticonceptivo. }\end{array}$ \\
\hline $\begin{array}{l}\text { Buena } \\
\text { práctica } \\
\text { clínica }\end{array}$ & $\begin{array}{l}\text { Se sugiere una visita a los tres meses tras el inicio de un tratamiento hormo- } \\
\text { nal combinado (oral, anillo o parche) o de sólo gestágeno, o a las 3-6 sema- } \\
\text { nas de la inserción en el caso del DIU. }\end{array}$ \\
\hline $\begin{array}{l}\text { Buena } \\
\text { práctica } \\
\text { clínica }\end{array}$ & $\begin{array}{l}\text { En las visitas de control, se sugiere comprobar cuál es su satisfacción con el } \\
\text { método y posibles problemas derivados de su uso. }\end{array}$ \\
\hline $\begin{array}{l}\text { Buena } \\
\text { práctica } \\
\text { clínica }\end{array}$ & $\begin{array}{l}\text { En las visitas de control, se sugiere explorar cualquier cambio en la historia } \\
\text { clínica de la usuaria que pueda causar un cambio en los criterios de elegibi- } \\
\text { lidad para el uso seguro de los anticonceptivos. }\end{array}$ \\
\hline Débil & $\begin{array}{l}\text { Se sugiere valorar cambios en el peso de la usuaria en cada visita de control } \\
\text { y discutir esta circunstancia con la usuaria que pueda asociar un eventual } \\
\text { cambio en el peso con el método anticonceptivo hormonal. }\end{array}$ \\
\hline Débil & $\begin{array}{l}\text { Se sugiere realizar una medición de la presión arterial en cada visita de con- } \\
\text { trol de las usuarias de anticonceptivos hormonales combinados (píldora, } \\
\text { parche o anillo). }\end{array}$ \\
\hline
\end{tabular}

\subsection{Manejo de las alteraciones del patrón de sangrado en anticoncepción}

\subsubsection{Valoración de las alteraciones del patrón de sangrado en usuarias de anticonceptivos hormonales y DIU}

El uso de anticonceptivos hormonales repercute en ocasiones en un cambio en el patrón de sangrado de las usuarias. Esto puede tener un impacto en el uso adecuado del anticonceptivo si el patrón de sangrado resulta insatisfactorio para la usuaria (110). Por este motivo las usuarias de estos anticonceptivos deben ser informadas de esta circunstancia. Teniendo en cuenta que los sangrados pueden ser frecuentes y prolongarse, por ejemplo, hasta los tres primeros meses de uso en el caso de los anticonceptivos hormonales, es necesario que antes de iniciar el tratamiento con un anticonceptivo y durante su uso se realice una anamnesis dirigida a caracterizar el patrón de sangrado de las usuarias y a descartar otras patologías.

La OMS establece unos criterios de definición del patrón de sangrado aplicable a los diferentes métodos anticonceptivos, utilizando periodos de referencia de 90 días en lugar de ciclos de 28 días. 
Tipo de sangrado en periodos de referencia de 90 días

\begin{tabular}{|l|l|}
\hline $\begin{array}{l}\text { Sangrado } \\
\text { regular }\end{array}$ & Tres episodios de sangrado o manchado de una duración de cinco días cada uno \\
\hline Amenorrea & No hay sangrado \\
\hline $\begin{array}{l}\text { Sangrado } \\
\text { infrecuente }\end{array}$ & Menos de dos episodios de sangrado o manchado \\
\hline $\begin{array}{l}\text { Sangrado } \\
\text { frecuente }\end{array}$ & Más de cuatro episodios de sangrado o manchado \\
\hline $\begin{array}{l}\text { Sangrado } \\
\text { irregular }\end{array}$ & $\begin{array}{l}\text { La diferencia entre el intervalo libre de sangrado más corto y el más largo es superior } \\
\text { a } 17 \text { días }\end{array}$ \\
\hline $\begin{array}{l}\text { Sangrado } \\
\text { prolongado }\end{array}$ & Al menos un episodio de sangrado o manchado de 10 días o superior \\
\hline
\end{tabular}

En la valoración del patrón de sangrado se pueden explorar aspectos como la preocupación de las usuarias por los sangrados no esperados, el tipo de sangrado y su patrón así como la comparación con patrones anteriores, el tipo de anticonceptivo que se utiliza o se desea utilizar, el empleo adecuado del anticonceptivo, otras circunstancias asociadas a sangrados no esperados. También se han propuesto algunas situaciones en las que puede ser necesario realizar una exploración ginecológica ante un sangrado no esperado: sospecha de otras patologías, mujeres mayores de 45 años, alto riesgo de ITS, presencia de otra sintomatología como dolor, dispareunia o sangrado postcoital, o en portadoras de DIU en las que se sospeche expulsión, infección o perforación $(8,111)$.

\subsubsection{Tratamiento de las alteraciones del patrón de sangrado en usuarias de anticonceptivos hormonales combinados en pauta extendida}

Una revisión sistemática ((112) fecha de búsqueda enero de 2012) evaluó las intervenciones para el manejo de los sangrados en usuarias de anticonceptivos hormonales combinados (píldora, parche o anillo).

En un ensayo abierto con 111 usuarias de anticonceptivos orales durante al menos 3 meses, se inició un régimen anticonceptivo extendido de 168 días de $3 \mathrm{mg}$ de drospirenona y $30 \mu \mathrm{g}$ de EE. Las mujeres que tuvieron un sangrado no esperado de más de siete días se aleatorizaron a un periodo libre de hormonas de tres días, o a continuar el régimen extendido de anticonceptivos (113). Los mismos investigadores evaluaron el manejo de los sangrados no esperados en las usuarias del anillo anticonceptivo (114). El estudio incluyó a 74 usuarias de anticonceptivos hormonales que iniciaron un tratamiento continuado de 6 meses con el anillo anticonceptivo. Al inicio del uso del anillo se aleatorizó a las usuarias a un intervalo libre de hormonas de cuatro días o a continuar con el ciclo cuando experimentaban un sangrado o manchado durante al menos cinco días.

Un ensayo doble ciego controlado con placebo evaluó el impacto sobre los sangrados no esperados de la doxiciclina en 66 mujeres que iniciaron un régimen extendido de anticonceptivos orales con levonorgestrel (115). Las mujeres debían tomar doxiciclina o placebo a partir del primer día del sangrado durante cinco días consecutivos. El tratamiento 
debía repetirse si las mujeres experimentaban un nuevo episodio de sangrado tras siete días de haber finalizado el primer tratamiento. Tras 84 días (tres ciclos de 28 días) las participantes debían tomar un último ciclo anticonceptivo pero sin tratamiento para los sangrados.

En un ensayo las mujeres con un periodo libre de hormonas resolvieron con mayor éxito el sangrado no esperado que las mujeres que continuaron con

Calidad baja el régimen extendido (17/30 (57\%) frente a $1 / 33(3 \%) ; \mathrm{P}<0,0001)(113)$.

Las mujeres en el grupo que tuvieron un periodo libre de hormonas experimentaron un incremento inicial del sangrado que disminuía de manera relevante al séptimo día y desaparecía al día 11; al final del estudio el patrón de sangrado fue menor en el grupo de mujeres que tuvieron un intervalo libre de hormonas que en el que siguió el régimen extendido.

En el ensayo del anillo (114), se registraron 50 sangrados o manchados en las mujeres que realizaron un intervalo libre de hormonas y 58 en las que realizaron el régimen continuo. A partir de los datos de un diario de sangrados de las usuarias, se observó que las mujeres que realizaban un intervalo libre de hormonas tenían un periodo mayor de ausencia de sangrado que las mujeres del régimen continuo (media de 95 días de ausencia de sangrado (rango de 92 a 97) frente a media de 89 días (rango de 85 a 93); $\mathrm{P}=$ $0,016)$. Las diferencias en los días con sangrado no fueron diferentes entre los grupos. Cuando se comparó el patrón de sangrado, las mujeres que realizaron un intervalo libre de hormonas experimentaron un incremento del sangrado en los tres primeros días tras la retirada del anillo, que disminuyó al octavo día, y al final del periodo de administración, el registro sobre el flujo fue inferior que en las mujeres que habían seguido con el anillo.

En el ensayo sobre la doxiciclina para el tratamiento de los sangrados no esperados (115), los investigadores no mostraron diferencias con el placebo en el total de días con sangrado o manchado $(23,1$ días (EE 2,2) frente a 19,2 (EE 3,4): $\mathrm{P}=0,32)$ o el total de días de sangrado (8,7 días (EE 2,2) frente a 6,5 (EE 1,6): $\mathrm{P}=0,63)$. La duración de los sangrados entre ambos grupos no fue diferente. Tampoco hubo diferencias en los días de sangrado durante el último ciclo de anticonceptivos que las mujeres debían tomar sin tratamiento para la alteración del ciclo $(3,2$ días $(\mathrm{EE} 0,9)$ frente a 2,9 (EE $1,0): \mathrm{P}=0,57)$.

\subsubsection{Tratamiento de las alteraciones del patrón de sangrado en usuarias de anticonceptivos de sólo gestágeno}

Una revisión sistemática Cochrane ((116) fecha de búsqueda mayo de 2012) evaluó las intervenciones para el manejo de los sangrados en usuarias de anticonceptivos de sólo gestágeno. 
La revisión incluyó 33 ensayos clínicos con un total de 3677 usuarias de anticonceptivos incluidas. Doce de estos ensayos evaluaron tratamientos en usuarias del implante subcutáneo de levonorgestrel y tres el de etonogestrel, tanto en nuevas usuarias como en usuarias de hasta un año. Otros doce ensayos evaluaron intervenciones en usuarias del inyectable de acetato de medroxiprogesterona y otro en usuarias del implante subcutáneo de levonorgestrel, con un periodo de uso de hasta 18 meses. Tres ensayos evaluaron intervenciones en usuarias de la píldora de sólo gestágeno. Finalmente dos ensayos evaluaron intervenciones en nuevas usuarias del DIU de levonorgestrel.

Los ensayos evaluaron hasta 18 intervenciones, 14 de ellas para el manejo de los sangrados no esperados. En siete ensayos se evaluó el uso de estrógenos solos y en tres ensayos más se probaron combinaciones de estrógenos con gestágeno. En dos ensayos se probó el impacto de antiprogestágenos como la mifepristona, que se combinaron con un estrógeno en dos ensayos más. En ocho ensayos se utilizaron antinflamatorios no esteroideos (AINE) como intervención terapéutica para el sangrado. Dos ensayos usaron vitamina E y otros dos usaron ácido tranexámico. Cuatro ensayos evaluaron inhibidores de la matriz de las metaloproteinasas (doxiciclina). Otra serie de intervenciones se evaluaron en un solo ensayo cada una: anticonceptivos hormonales combinados con levonorgrestel, un venotónico, una combinación de vitamina $\mathrm{E}$ con un AINE, un modulador selectivo de los receptores estrogénicos.

A pesar de que los ensayos tenían como desenlace de interés el tratamiento de los episodios de sangrado, la medida del resultado fue muy variable entre los estudios incluidos. Los ensayos también evaluaron la interrupción del tratamiento anticonceptivo, los efectos adversos derivados del tratamiento, o la insatisfacción con el tratamiento. La variabilidad en los estudios provocó que los autores de la revisión pudieran combinar en pocas ocasiones los resultados de los estudios incluidos, contribuyendo a la dificultad en la interpretación de los resultados. Dos tercios de los estudios incluidos en la revisión tuvieron un riesgo de sesgo alto (por limitaciones en el encubrimiento de la asignación o un sesgo de desgaste importante) o no ofrecían suficiente información como para determinar su riesgo de sesgo.

Los estudios que evaluaron el uso de estrógenos en el manejo de las alteraciones del ciclo en usuarias del implante subcutáneo de levonorgestrel o el Calidad inyectable de acetato de medroxiprogesterona fueron inconsistentes. Los estrógenos redujeron el sangrado irregular en usuarias del implante ( 2 ensayos; RR 0,43; IC95\% 0,30 a 0,61). Los resultados fueron similares en un ensayo con usuarias del inyectable pero los datos del estudio no se pudieron extraer correctamente para el análisis en la revisión de Abdel-Aleem 2013 (116). Sin embargo, el tratamiento también repercutió en una mayor tasa de abandonos de los estudios por efectos adversos comparados con sus controles (2 ensayos; RR 8,10; IC95\% 1,04 a 63,40). 
En dos ensayos se evaluó el tratamiento con anticonceptivos orales combinados de levonorgestrel en usuarias de un implante subcutáneo de levonorgestrel con sangrados. Los dos estudios describieron un efecto beneficioso del anticonceptivo oral para parar el sangrado, aunque solamente se pudieron analizar los datos de uno de ellos (RR 0,08; IC95\% 0,03 a 0,24). No se observaron problemas con los efectos adversos relevantes como en el caso de los ensayos en los que se administraron estrógenos sólos. En un ensayo pequeño (46 mujeres) un anticonceptivo oral combinado también redujo los sangrados en usuarias del inyectable de acetato de medroxiprogesterona (RR 0,38; IC95\% 0,19 a 0,73), que repercutió en un porcentaje menor de mujeres que interrumpieron el tratamiento anticonceptivo (RR 0,60; IC 95\% 0,40 a 0,88).

La mifepristona se utilizó parar tratar los sangrados en las usuarias de un implante subcutáneo de levonorgestrel en un ensayo clínico que mostró una reducción significativa de la duración de los sangrados durante los seis primeros meses de duración del tratamiento (100 mujeres, DM 6,5 días menos de sangrado; IC 95\% de 3 a 10 días menos). El tratamiento con un inhibor selectivo del receptor de progesterona también se mostró beneficioso en el tratamiento de los sangrados en usuarias del inyectable de acetato de medroxiprogesterona (1 ensayo), la píldora de sólo gestágeno (1 ensayo) y el DIU de levonorgestrel (1 ensayo). Sin embargo este tratamiento no se mostró eficaz en un ensayo en el que se evaluó su eficacia para el manejo de las alteraciones del sangrado en usuarias del implante subcutáneo de etonogestrel, aunque cuando se añadió un estrógeno a la mifepristona los resultados sugirieron una reducción de los episodios de sangrado ( 77 mujeres; RR 0,04; IC95\% 0,00 a 0,66). Los autores de la revisión sistemática advierten que la seguridad de este tratamiento necesita evaluarse con más rigor.

Un ensayo con una muestra limitada (100 mujeres) evaluó el tamoxifeno para tratar las alteraciones del ciclo en usuarias del implante subcutáneo de levonorgestrel mostrando una reducción significativa de los sangrados (RR 0,41 ; IC95\% 0,21 a 0,80) y de las mujeres que abandonaban el método anticonceptivo por un sangrado (RR 0,22; IC95\% 0,05 a 0,98) al compararlo con placebo. 
Otros tratamientos han mostrado un efecto moderado en ensayos clínicos pequeños, resultados que no se han confirmado en estudios más robustos, por lo que la aplicabilidad de los resultados debe tomarse con cautela

Calidad muy baja (116). Por ejemplo, mientras que la vitamina $\mathrm{E}$ redujo el número de días con sangrado en un estudio piloto en usuarias de un implante subcutáneo de levonorgestrel, los resultados no se replicaron en un estudio posterior con mayor tamaño muestral. Este mismo estudio tampoco confirmó los resultados favorables observados con el tratamiento con AINES en un ensayo clínico con ibuprofeno en 21 mujeres, uno sobre ácido mefenámico en 34 mujeres, otro sobre valdecoxib en 22 mujeres, o un último ensayo sobre naproxeno en 42 nuevas usuarias del DIU de levonorgestrel. Por último, el ácido tranexámico, la mifepristona combinada con un estrógeno y la doxiciclina mostraron un mejor efecto que el placebo en la interrupción de los sangrados en tres estudios pequeños.

\subsubsection{Tratamiento de las alteraciones del patrón de sangrado en usuarias de DIU de cobre}

Una revisión sistemática ((117) fecha de búsqueda marzo de 2012) evaluó las intervenciones para el manejo de los sangrados en usuarias de DIU de cobre. La revisión incluyó una serie de estudios que están incluidos en una revisión Cochrane (118; actualización de la búsqueda en agosto de 2011). Se han recopilado los datos de esta última revisión sistemática ya que presenta los datos de una manera más exhaustiva.

Una revisión incluyó ocho ensayos clínicos que evaluaron el efecto de diferentes AINE de los que seis aportaron datos para el sangrado (118). Un ensayo en 48 usuarias de DIU (34 de cobre) evaluó una dosis diaria naproxeno de 1250mg dividida en varias tomas, una dosis de 1 gr en el primer día de tratamiento seguida de dosis diarias de $750 \mathrm{mg}$ y placebo. En otro ensayo en 24 usuarias de DIU de cobre se comparó un tratamiento con desmopresina nasal durante cinco días iniciado el primer día de sangrado frente al ácido mefenámico de $1,5 \mathrm{gr}$ diarios con la misma duración. Un estudio comparó un tratamiento de siete días de indometacina $50 \mathrm{mg}$ que empezaba el primer día de sangrado frente a un preparado natural herbal (baofuxin $16 \mathrm{gr}$ ). La indometacina también se comparó con placebo en un ensayo cruzado en 15 mujeres. Otro ensayo de diseño cruzado en 28 mujeres comparó suporfeno frente a placebo. Finalmente un ensayo en 31 mujeres comparó con placebo un tratamiento con ácido tranexámico (4,5 g diarios durante cinco días empezando el primer día de sangrado), o uno con diclofenaco (150 mg el primer día de sangrado y $75 \mathrm{mg}$ en los cuatro días posteriores).

Una revisión describe que el naproxeno frente al placebo reduce el sangrado en usuarias de un DIU de cobre (pérdida de sangre pre-tratamiento:

Calidad baja $127 \mathrm{~mL}$; con naproxeno $1250 \mathrm{mg}$ : $93 \mathrm{~mL}$; con naproxeno $750 \mathrm{mg}$ : $89 \mathrm{~mL}$; con placebo: $129 \mathrm{~mL}$ ) (118). No se observaron efectos adversos relevantes. 
El ácido mefenámico y la desmopresina nasal mostraron resultados similares con una reducción similar del sangrado percibido por las mujeres en relación con el inicio del estudio ( $46 \%$ frente a $41 \%$ ), con respuestas al tratamiento variables y sin que se observaran efectos adversos graves (3/12 mujeres tratadas con desmopresina aquejaron de insomnio y cefalea). Grimes 2006 argumenta que los resultados del ácido mefenámico pueden ser más favorables, teniendo en cuenta el mayor coste de la desmopresina (118).

Un ensayo mostró que la indometacina conseguía una reducción de los días de sangrado similar a las de un remedio natural (baofuxin) (media a 28,8 días frente a 28,3 días), así como las ocasiones en las que las mujeres registraban un sangrado (media de 3,4 frente a 3,3). La proporción de mujeres a las que se les extrajo el DIU por sangrado también fue similar (13 frente a 12). El ensayo que comparó la indometacina con placebo mostró una reducción del sangrado respecto al inicio del estudio del 57\% (de 174,7 mL a $75,1 \mathrm{~mL}$ ), sin observar efectos adversos notables.

El suprofeno consiguió una reducción que las mujeres consideraron notable en 22/28 de las tratadas con este AINE, mientras que ninguna de las mujeres tratadas con placebo percibió este cambio. Dos de las mujeres que recibieron suprofeno sufrieron náuseas y cefaleas.

El ácido tranexámico mostró mejores resultados que el diclofenaco, con una reducción del sangrado del $50 \%$ y el $20 \%$ respectivamente. Mientras que las usuarias del ácido tranexámico reportaron problemas gastrointestinales y cefaleas, las que tomaron diclofenaco reportaron fatiga, sequedad labial, picores o edema (una de las mujeres abandonó el estudio por esta causa).

\section{De la evidencia a la recomendación}

\section{Resumen de la evidencia}

Un intervalo libre de hormonas breve (entre tres y cuatro días) durante el uso de anticonceptivos combinados orales o el anillo, reduce la frecuencia de sangrados $(113,114)$.

La doxiciclina no ha mostrado un beneficio comparada con placebo en el tratamiento de los sangrados en usuarias de anticonceptivos combinados orales (115).

La literatura científica ha mostrado el beneficio de algunos tratamientos para el manejo de las alteraciones del ciclo en las usuarias de anticonceptivos de sólo gestágeno, aunque las limitaciones de los estudios disponibles

Calidad baja

Calidad baja

Calidad baja relacionados con las diferencias existentes entre ellos y su tamaño limitado hacen que se desconozca el efecto de estos tratamientos a largo plazo y su relevancia clínica (116). 
El análisis de estos estudios han mostrado resultados variados, que podrían sintetizarse de la siguiente manera: i) el tratamiento con estrógenos ha mostrado una reducción de los días con sangrados en las usuarias del implante y el inyectable, aunque sus efectos adversos derivaron en los estudios en más abandonos; ii) este efecto también se ha observado con un anticonceptivo oral combinado en usuarias del implante e inyectable; iii) la mifepristona ha mostrado resultados inconsistentes en las usuarias del implante; iv) los AINE han mostrado resultados muy variados en estudios pequeños; v) el tamoxifeno ha mostrado un leve beneficio en usuarias del implante; vi) intervenciones como el ácido tranexámico, la combinación de mifepristona con un estrógeno o la doxiciclina han mostrado un efecto leve en estudios con una muestra pequeña (116). Es necesario destacar que alguno de estos tratamientos no se encuentran disponibles en el Sistema Nacional de Salud.

Los AINE han mostrado que reducen los sangrados en las usuarias del DIU de cobre, aunque la variedad de estudio y sus limitaciones no permiten destacar a uno sobre los otros. El naproxeno, la indometacina, el suprofeno y baja el diclofenaco han mostrado mejores resultados que el placebo. El ácido mefenámico ha mostrado unos resultados similares a la desmopresina. Finalmente, aunque el ácido tranexámico reduce en una proporción mayor los sangrados que el diclofenaco y el placebo, produce efectos adversos menos tolerables (118).

\section{Calidad de la evidencia}

La calidad global es baja.

Para el manejo de las alteraciones del patrón de sangrado en usuarias de anticonceptivos hormonales combinados se ha considerado que la calidad es baja debido a que los dos principales ensayos fueron abiertos, utilizaron medidas de resultado no validadas (diarios de autoregistro de intensidad de sangrado) y tuvieron muestras pequeñas. En el caso de las intervenciones para usuarias de anticonceptivos de sólo gestágeno la calidad también fue baja por las limitaciones en el diseño de los estudios (dos tercios de los ensayos tenían un riesgo de sesgo alto o incierto) y por la inconsistencia de sus hallazgos (la multitud de intervenciones evaluadas e inconsistencia en la medida y descripción de los resultados de interés en los estudios incluidos dificultaron la interpretación y aplicabilidad de los hallazgos de la revisión).

La calidad de la evidencia para el tratamiento con AINE en usuarias de DIU de cobre también es baja. Grimes 2006 destaca las limitaciones metodológicas de los ensayos clínicos disponibles, en muchas ocasiones con un diseño cruzado, y con un tamaño de la muestra muy reducido, con un impacto directo en la previsión de los resultados (118).

\section{Valores y preferencias de las usuarias}

El cambio en el patrón de sangrado de las usuarias de anticonceptivos hormonales repercute en la satisfacción con el tratamiento y su continuidad. Un estudio valoró la po- 
sible relación entre una alteración en el patrón de manchado y la interrupción del uso de los anticonceptivos hormonales analizando las razones de abandono de cinco ensayos clínicos como una aproximación a la experiencia de las usuarias (110). Las usuarias del anillo y de los anticonceptivos hormonales combinados que interrumpían su participación en los ensayos, tenían patrones de sangrado similares a las mujeres que continuaban en el estudio. En cambio las usuarias del inyectable que abandonaron el estudio acostumbraban a hacerlo por las alteraciones en su patrón de sangrado. Las mujeres que interrumpieron la participación en el ensayo por una amenorrea tenían pocos días con manchados o sangrados inesperados y al menos un intervalo sin sangrado prolongado. La principal alteración del patrón de sangrado en las participantes en el estudio fue la frecuencia de los sangrados inesperados.

\section{Beneficios y riesgos/carga de las intervenciones}

No se ha considerado este aspecto en esta pregunta clínica.

\section{Balance beneficio/riesgo}

No se ha considerado este aspecto en esta pregunta clínica.

\section{Uso de recursos y costes}

No se ha considerado este aspecto en esta pregunta clínica.

\section{Justificación}

Las recomendaciones en esta pregunta clínica se fundamentan en la necesidad de realizar un manejo de las alteraciones del patrón de sangrado de las usuarias de anticonceptivos hormonales e intrauterinos que permitan asegurar una buena adherencia a su uso, preservando su eficacia. Las únicas recomendaciones que pueden basarse en los resultados de la literatura son los relativos al tratamiento de los sangrados inesperados. Se ha sintetizado en el Algoritmo 2 la actuación ante la alteración del patrón de sangrado en anticoncepción hormonal o intrauterina. 


\section{Recomendaciones}

\begin{tabular}{|l|l|}
\hline $\begin{array}{l}\text { Buena } \\
\text { práctica } \\
\text { clínica }\end{array}$ & $\begin{array}{l}\text { Antes de iniciar el tratamiento con un anticonceptivo hormonal o intrauteri- } \\
\text { no debe informarse a la usuaria de la modificación en su patrón de sangrado. } \\
\text { Principalmente en el caso de los dispositivos intrauterinos y los métodos } \\
\text { hormonales combinados, la modificación del patrón del sangrado puede ser } \\
\text { habitual al inicio del uso del método, y tiende a desaparecer con su uso con- } \\
\text { tinuado. }\end{array}$ \\
\hline $\begin{array}{l}\text { Buena } \\
\text { práctica } \\
\text { clínica }\end{array}$ & $\begin{array}{l}\text { En el proceso de elección de un anticonceptivo hormonal o intrauterino } \\
\text { debe realizarse una anamnesis orientada a identificar factores de riesgo u } \\
\text { otras situaciones que permitan anticipar alteraciones en el patrón de san- } \\
\text { grado. }\end{array}$ \\
\hline Débil & $\begin{array}{l}\text { En usuarias de implante o inyectable se sugiere que ante la persistencia del } \\
\text { sangrado, y en ausencia de problemas ginecológicos, se trate con antinfla- } \\
\text { matorios no esteroideos. En caso de sangrado frecuente o prolongado se su- } \\
\text { giere un tratamiento con anticonceptivos combinados o estrógenos en dosis } \\
\text { bajas. }\end{array}$ \\
\hline Débil & $\begin{array}{l}\text { En usuarias de anticonceptivos hormonales combinados se sugiere que ante } \\
\text { la persistencia del sangrado, y en ausencia de problemas ginecológicos, se } \\
\text { interrumpa el tratamiento durante } 3 \text { ó } 4 \text { días consecutivos. Estos días de } \\
\text { descanso del anticonceptivo, no están indicados en los primeros 21 días de } \\
\text { uso del método hormonal combinado en ciclos extendidos, ni está indicado } \\
\text { realizarlos en más de una ocasión en el mismo mes, ya que el anticonceptivo } \\
\text { puede reducir su eficacia. }\end{array}$ \\
\hline
\end{tabular}

\subsection{Eventualidades en el uso de la anticoncepción}

\subsubsection{Manejo de los olvidos de las píldoras combinadas y de solo gestágeno}

Una revisión sistemática no ha identificado estudios que evalúen cómo los olvidos en la toma de las píldoras anticonceptivas influyen en el riesgo de embarazo (119), y centra su atención en estudios que evalúan la función ovárica en ciclos en los cuáles los periodos libres de hormona se extendían deliberadamente, o en ciclos en los que se olvidaba deliberadamente la toma de una píldora en días que no estaban próximos a la semana libre de toma.

Los estudios mostraban un impacto muy variable en el desarrollo folicular y la ovulación en las usuarias de anticonceptivos hormonales orales que extienden el periodo libre de hormonas hasta los 14 días. En términos generales pocas mujeres en este grupo ovulaban, y entre las que lo hacían, los ciclos eran irregulares (niveles de progesterona menores, folículos pequeños, poco moco cervical). Los estudios en los que había un olvido de entre una y cuatro píldoras en días que no estuvieran próximos al intervalo libre de hormona mostraban una actividad folicular baja y un riesgo de ovulación bajo. Sin lugar a dudas, es difícil establecer una relación entre estos desenlaces intermedios y el impacto de los olvi- 
dos en la eficacia de los anticonceptivos. Sin embargo, el uso incorrecto de los anticonceptivos hormonales tiene un impacto sobre su eficacia, por lo que habría cantidades y momentos del ciclo donde los olvidos aumentarían el riesgo de embarazo.

Se han identificado cuatro documentos que abordan el manejo de los olvidos de anticonceptivos orales, que incluyen los anticonceptivos combinados y los de sólo gestágeno $(3,120-122)$.

Una revisión de la literatura que resume las reglas a seguir cuando se olvida una o más de una píldora (120), recoge las recomendaciones sobre esta situación de las principales agencias reguladoras y de evaluación: Faculty of Sexual and Reproductive Healthcare (121), y la Medicines and Healthcare products Regulatory Agency (123). Por otro lado, el documento del Centers for Disease Control and Prevention (3), ofrece una propuesta simplificada para el manejo de los olvidos basado en una revisión sistemática (119).

Las directrices recogidas en estos documentos se basan en un balance entre los resultados derivados de la literatura y el criterio clínico de los expertos, por lo que no es posible realizar una evaluación formal de la calidad de la evidencia. Las recomendaciones derivadas de este apartado (mostradas en el Algoritmo 3) se consideran recomendaciones de buena práctica clínica. Para el manejo de los olvidos de las píldoras de solo gestágeno se muestran las indicaciones en el Algoritmo 4.

Una revisión evaluó la comprensión de las usuarias de las instrucciones sobre cómo actuar tras el olvido de las píldoras anticonceptivas ((124) fecha de búsqueda en marzo de 2012), incluyendo nueve estudios sobre el conocimiento y comprensión de estas instrucciones tras recibir información sobre el tema en diversos formatos, de los cuales tres fueron ensayos clínicos (125 - 127).

Los resultados de los estudios incluidos en la revision mostraron que las mujeres tenían un mejor conocimiento sobre cómo actuar tras el olvido

Calidad baja de una píldora que tras haber olvidado dos o tres píldoras. Dos estudios transversales mostraron que las mujeres tenían dificultades para entender la información sobre los olvidos en los prospectos de los envases.

Los ensayos incluidos mostraron que el consejo anticonceptivo combinado con un folleto informativo con instrucciones sobre como actuar ante un olvido, mejoró el conocimiento de las usuarias comparado con el consejo anticonceptivo solo (125 - 127).

Uno de los estudios, en 1239 mujeres pero con un seguimiento de solamente un tercio de las participantes, comparó la provisión de información oral en la consulta anticonceptiva frente a la combinación de un folleto informativo o un folleto y una grabación (127). El apoyo audiovisual mejoró el conocimiento sobre la eficacia de los anticonceptivos y la ventaja de su uso correcto, así como el conocimiento sobre cómo actuar ante un olvido. 
Otro estudio también valoró el beneficio de entregar folletos informativos en 636 mujeres que asistían a un centro de salud para una prescripción de anticonceptivos (126), mediante un cuestionario que respondieron el $80 \%$ de las participantes. La intervención mejoró el conocimiento sobre las consecuencias de un olvido en las mujeres que recibieron el folleto informativo ( $22 \%$ frente a $12 \% ; \mathrm{P}<0,01)$. La provisión de folletos informativos (OR 4,04; IC95\% 1,68 a 9,75) y la respuesta a preguntas (OR 3,03; IC95\% $1,30$ a 7,00$)$ mostraron una mejora significativa de la comprensión sobre los aspectos relacionados con los olvidos.

Un último ensayo clínico en 864 usuarias de las que solamente el $60 \%$ respondió a un cuestionario, mostró que las mujeres que recibieron materiales informativos mejoraron la comprensión sobre cómo actuar ante un olvido que ante dos o más olvidos. Por otro lado, la información gráfica era más fácil de entender que la que se basaba solamente en texto (127).

Por otro lado, una revisión sistemática Cochrane ((122) fecha de búsqueda en 2013) evaluó intervenciones para mejorar el cumplimiento con los anticonceptivos orales, incluyendo el olvido como uno de los desenlaces de interés.

La revisión tiene como objetivo comparar la eficacia de diferentes técnicas para mejorar la adherencia al tratamiento de las mujeres usuarias de anticonceptivos hormonales. Esta revisión incluye los resultados de un ensayo clínico (128) realizado en un centro de planificación familiar de EEUU en el que 42 mujeres recibieron recordatorios diarios mediante mensajes de texto y otras 42 mujeres no recibieron ningún mensaje. El ensayo evaluó el número de olvidos por ciclo. La media de edad de las mujeres fue de 22 años, que en su mayoría fueron mujeres blancas no hispanas (79\%), con estudios secundarios $(99 \%)$ y nulíparas (99\%). La mayoría de las participantes había solicitado la anticoncepción de urgencia anteriormente. En otro ensayo clínico se evaluó el impacto de enviar mensajes de texto a diario (recordatorios para tomar el anticonceptivo, consejos sobre diferentes aspectos de la anticoncepción oral combinada) frente al consejo anticonceptivo habitual en 925 mujeres menores de 25 años que asistían a un centro de planificación familiar en Nueva York (129). Aunque el ensayo tenía como objetivo principal comprobar el cumplimiento con el tratamiento (tomas de anticonceptivos durante la última semana), también se registró la tasa de olvidos.

Un ensayo clínico no mostró que un recordatorio diario mediante telefonía móvil repercutiera en el número de olvidos de las usuarias de anticonceptivos (128). El grupo que recibió los recordatorios (36 mujeres) tuvo una

Calidad baja media de 4 olvidos (DE 3,5), mientras que las mujeres que no los recibieron (37 mujeres) tuvo una media de 3,5 olvidos (DE 3,4) (DM: 0,50; IC95\% $-1,08$ a 2,08$)$.

En un ensayo clínico las mujeres que recibían mensajes de texto olvidaban en un porcentaje menor tomar la píldora durante más de siete días que las mujeres que recibían consejo anticonceptivo habitual (683 mujeres; OR 1,53; IC95\% 1,13 a 2,07) (129). 


\section{De la evidencia a la recomendación}

\section{Resumen de la evidencia}

No se ha establecido de manera directa la relación entre el olvido de la toma de los anticonceptivos hormonales orales y su eficacia. Estudios que han evaluado el impacto de un olvido de entre una y cuatro píldoras en días que no estuvieran próximos al intervalo libre de hormona han mostrado una actividad folicular baja y un riesgo de ovulación bajo (119).

La comprensión de las instrucciones sobre cómo actuar ante un olvido es variable entre los estudios, aunque las usuarias tienen más conocimiento de qué hacer tras un olvido, que tras haber olvidado dos o tres píldoras. Las usuarias parecen tener dificultades en interpretar las instrucciones sobre los olvidos que incluyen los prospectos de los envases, por lo que el consejo sobre cómo actuar ante tales situaciones acompañado de información escrita presentada de manera gráfica y sencilla puede mejorar la comprensión (124).

El uso de recordatorios a las usuarias de la píldora anticonceptiva ha mostrado resultados discrepantes. Mientras que un ensayo mostró que un sisteCalidad baja ma diario de recordatorio mediante mensaje de texto no reducía de manera relevante el número de olvidos (127), un sistema más intensivo de mensajes de recordatorio y consejos sobre la anticoncepción redujo el porcentaje de mujeres que olvidaban la toma durante más de siete días (129).

\section{Calidad de la evidencia}

La calidad global es baja para la relación entre los olvidos y la eficacia de los anticonceptivos, y para las intervenciones dirigidas a reducir los olvidos.

En el primer caso, todos los estudios de una revisión han evaluado el tema de interés de manera indirecta (124). En cuanto a las intervenciones para reducir los olvidos, los ensayos clínicos evaluados muestran resultados discrepantes y tuvieron limitaciones en el diseño importantes: Hou 2010 tenía un tamaño de muestra muy limitado (83 participantes) (128) y Castaño 2012 era un estudio abierto que sufrió un número considerable de pérdidas (129). El resto de estudios han evaluado intervenciones muy diferentes entre ellas y han medido los resultados mediante cuestionarios con una baja tasa de respuesta (un tercio en Deijen 1997 (125), y 60\% en Chin-Quee 2006 (127)).

\section{Valores y preferencias de las usuarias}

En una revisión sistemática sobre los atributos que influyen la elección de un anticonceptivo por parte de una mujer, la posibilidad de un olvido apareció como un aspecto determinante en un tercio de los estudios que realizaron una entrevista o recabaron los datos 
mediante un cuestionario ( $7 / 20$ ), y en un $40 \%$ de los estudios que aplicaban un instrumento de decisión compartida (8/19) (37).

\section{Beneficios y riesgos/carga de las intervenciones}

No se ha considerado este aspecto en esta pregunta clínica.

\section{Balance beneficio/riesgo}

Aunque no se ha establecido de manera directa el impacto de los olvidos sobre la eficacia de los anticonceptivos, la cantidad de píldoras olvidadas y el momento en el que se olvida la toma pueden aumentar el riesgo de un embarazo no deseado. Este hecho justifica la necesidad de establecer unos criterios que permitan retomar de la manera más eficiente posible el uso de los anticonceptivos.

\section{Uso de recursos y costes}

No se ha considerado este aspecto en esta pregunta clínica.

\section{Justificación}

En la formulación de una recomendación sobre directrices para el manejo de los olvidos de las píldoras anticonceptivas, debe tenerse en cuenta que éstas se basan en un balance entre los resultados de la literatura y el criterio clínico, por lo que las recomendaciones derivadas se consideran recomendaciones de buena práctica clínica.

\section{Recomendaciones}

\begin{tabular}{|l|l|}
\hline $\begin{array}{l}\text { Buena } \\
\text { práctica } \\
\text { clínica }\end{array}$ & $\begin{array}{l}\text { Durante una consulta de anticoncepción, cada profesional sanitario debería } \\
\text { informar sobre el posible impacto de un olvido y responder a las posibles } \\
\text { dudas de cada usuaria, valorando la posibilidad de proporcionar folletos in- } \\
\text { formativos sobre este aspecto. }\end{array}$ \\
\hline $\begin{array}{l}\text { Buena } \\
\text { práctica } \\
\text { clínica }\end{array}$ & $\begin{array}{l}\text { En caso de que se produzca un olvido en la toma de un anticonceptivo hor- } \\
\text { monal oral combinado, se sugiere considerar el momento en el que se produ- } \\
\text { jo el olvido y el número de píldoras olvidadas, para aconsejar a la usuaria la } \\
\text { manera más adecuada de retomar el uso adecuado del anticonceptivo (con- } \\
\text { sultar Algoritmo 3). }\end{array}$ \\
\hline $\begin{array}{l}\text { Buena } \\
\text { práctica } \\
\text { clínica }\end{array}$ & $\begin{array}{l}\text { En caso de que se produzca un olvido en la toma de un anticonceptivo hor- } \\
\text { monal de solo gestágeno, se sugiere considerar el momento en el que se } \\
\text { produjo el olvido, para aconsejar a la usuaria la manera más adecuada de } \\
\text { retomar el uso adecuado del anticonceptivo (consultar Algoritmo 4). }\end{array}$ \\
\hline
\end{tabular}




\subsubsection{Anticoncepción hormonal e interacciones medicamentosas}

Un documento de recomendaciones de la Faculty of Sexual and Reproductive Healthcare - FSRH (130) recopila consideraciones ante la prescipción de medicamentos a mujeres que reciben un tratamiento con anticonceptivos hormonales. La literatura científica que evalúa la interacción de fármacos con los anticonceptivos hormonales es escasa y de baja calidad, ya que procede de descripciones de casos, series de casos muy pequeñas, $o$ alertas de fármacovigilancia. El documento de la FSRH realiza una síntesis exhaustiva, actual y rigurosa sobre estos estudios.

Las interacciones medicamentosas se producen cuando la eficacia contraceptiva de un anticonceptivo se ve alterada por el mecanismo de acción de algún otro medicamento. La eficacia del anticonceptivo puede verse alterada por cambios en su farmacocinética o famacodinámica. El primer aspecto está relacionado con la alteración de los mecanismos de absorción, distribución, metabolismo o secreción del medicamento, afectando a la concentración del anticonceptivo. La farmacodinámica se ve afectada cuando un fármaco influye directamente en la acción clínica del anticonceptivo (por sinergia o antagonismo).

El documento de recomendaciones del FSRH (130), contempla aspectos relacionados con la información a discutir en el momento en el que se prescribe un medicamento a una mujer que está tomando un anticonceptivo hormonal, describe los fármacos que tienen una potencial influencia en la eficacia de los anticonceptivos hormonales, ofrece consejos para las mujeres que usan fármacos con interacciones sobre la eficacia de los anticonceptivos hormonales, y describe los principales tratamientos que aumentan la concentración de los anticonceptivos hormonales.

Por otro lado los documentos para determinar los criterios médicos de elegibilidad para el uso de anticonceptivos incluyen en su mayoría un apartado en los que se contemplan las principales interacciones medicamentosas, y situaciones en las que un embarazo aumenta el riesgo de las mujeres con determinados problemas de salud (como por ejemplo, epilepsia, diabetes, tuberculosis, HIV, cardiopatía coronaria, etc.) (3 - 5).

\subsubsection{Aspectos a discutir en el momento de prescribir fármacos a una mujer que esté tomando un anticonceptivo hormonal}

Siempre debe tenerse en cuenta la posibilidad de una interacción medicamentosa en el momento de prescribir un anticonceptivo hormonal (incluso en anticoncepción de emergencia), o en el momento de prescribir un tratamiento a una mujer que está tomando un anticonceptivo hormonal (130).

Se debe advertir a las mujeres que están utilizando un anticonceptivo hormonal que ciertos medicamentos pueden alterar la eficacia del anticonceptivo y, por lo tanto, que puede ser necesario tomar alguna medida anticonceptiva adicional. La duración del tratamiento concomitante, y la causa por la que se debe prescribir este tratamiento, pueden ser aspectos determinantes en el momento de decidir qué anticonceptivo administrar. El Anexo 2 recoge los fármacos que pueden reducir los niveles de hormonas anticonceptivas o la eficacia contraceptiva de estos tratamientos. 


\subsubsection{Fármacos con una interacción sobre la eficacia contraceptiva de los anticonceptivos hormonales}

\subsection{Fármacos que producen inducción enzimática}

Los fármacos inductores enzimáticos (como algunos antiepilépticos como la carbamazepina o la fenitoína) reducen la eficacia contraceptiva de los anticonceptivos hormonales, excepto en el caso de los inyectables de sólo progestágeno (DMPA y NET-EN) y el dispositivo intrauterino de levonorgestrel (DIU LNG) (130). Estos fármacos pueden aumentar el metabolismo de los estrógenos y/o el progestágeno, reduciendo la biodisponibilidad del anticonceptivo y reduciendo su eficacia.

Aunque la lamotrigina no induce la actividad enzimática, una pequeña serie de casos en 16 mujeres sanas (131) mostró que la toma de este fármaco conjuntamente con un anticonceptivo combinado oral con levonorgestrel no afectó los niveles de etinilestradiol, aunque los niveles de concentración de levonorgestrel disminuyeron de forma moderada. Este hecho se asociaba a un aumento de las hormonas FSH y LH y un incremento de los sangrados intermenstruales. No obstante la eficacia contraceptiva del anticonceptivo se mantuvo.

\subsection{Fármacos con un efecto sobre la absorción de otros fármacos}

Algunos fármacos alteran la absorción de otros fármacos al alterar el nivel de $\mathrm{pH}$ gástrico. Los fármacos que aumentan el $\mathrm{pH}$ gástrico (como los antiácidos, antagonistas de los receptores $\mathrm{H} 2$, o los inhibidores de la bomba de protones), pueden reducir la absorción y eficacia del ulipristal utilizado como anticonceptivo de urgencia (132).

Por otro lado, en ocasiones ciertas medicaciones pueden inducir el vómito o provocarlo como efecto secundario. Las mujeres que vomitan durante las dos horas siguientes a haber tomado un contraceptivo oral, deberían repetir la dosis lo antes posible (133).

\subsection{Antibióticos que no alteran la actividad enzimática}

La rifampicina y la rifabutina son los únicos antibióticos que han mostrado una acción sobre la actividad enzimática, y que alteran la concentración de etinilestradiol (134-138).

En la literatura también se ha descrito el fallo terapéutico de los anticonceptivos cuando se han administrado de manera concomitante con otros antibióticos sin ninguna acción sobre la actividad enzimática como la penicilina, tetraciclinas, macrólidos, fluoroquinolonas o el antifúngico imizadol (139-154). A pesar de estos datos recogidos en la literatura, los criterios de elegibilidad de la Organización Mundial de la Salud (4) consideran que la eficacia contraceptiva de los anticonceptivos hormonales no se ve afectada por la mayoría de antibióticos de amplio espectro (tetraciclinas, macrólidos y cloranfenicol), y por lo tanto pueden administrarse sin restricción y sin necesidad de recomendar precauciones anticonceptivas adicionales. 


\subsubsection{Consejos a las mujeres que toman un fármaco con una interacción sobre la eficacia contraceptiva de los anticonceptivos hormonales}

\subsection{Fármacos que producen inducción enzimática}

Se debería aconsejar a las mujeres que toman algún fármaco inductor de la actividad enzimática a que escoja algún método anticonceptivo que no esté afectado por este tipo de fármaco. Los anticonceptivos inyectables de sólo progestágeno y el dispositivo intrauterino de levonorgestrel no han mostrado una interacción con estos fármacos (130).

\subsection{Fármacos que producen inducción enzimática - Anticonceptivos hormonales combinados}

Si una mujer toma un método combinado y no quiere cambiar a un método que no esté afectado por los fármacos inductores de la actividad enzimática, debe considerar alguna de las siguientes opciones.

- Si la mujer ha usado el fármaco inductor de la actividad enzimática durante un periodo breve de tiempo (menos de dos meses), debe continuar con el método combinado (sea en forma de parche, anillo, o la píldora con una dosis mínima de $30 \mu \mathrm{g}$ de etinilestradiol), pero se le debe aconsejar el uso de precauciones anticonceptivas adicionales (preservativo) mientras continúe tomando el fármaco inductor y hasta los 28 días después de la interrupción del tratamiento.

- Si la mujer ha usado el fármaco inductor de la actividad enzimática durante un periodo superior a los dos meses, se debe recomendar un aumento de la dosis del anticonceptivo a una dosis de al menos $50 \mu \mathrm{g}$ de etinilestradiol mientras continúe tomando el fármaco inductor y hasta los 28 días después de la interrupción del tratamiento.

- Si la mujer está tomando rifampicina o rifabutina, fármacos con una fuerte acción sobre la inducción de la actividad enzimática, se debe aconsejar que se cambie a un método que no esté afectado por la acción de estos fármacos.

\subsection{Fármacos inductores enzimáticos - Anticonceptivos con sólo progestágeno}

Las mujeres que toman fármacos inductores de la actividad enzimática y usan el método anticonceptivo de sólo progestágeno inyectable (DMPA y NET-EN) o el DIU de levonorgestrel (DIU LNG) no requieren de precauciones adicionales, ajustes de dosis, o modificaciones en la pauta de administración.

Se debe informar a las usuarias de la píldora de sólo progestágeno o el implante que los fármacos inductores de la actividad enzimática pueden interactuar con la eficacia del método y por tanto deben considerar utilizar un método alternativo que no esté afectado por la acción de estos fármacos. 


\subsection{Fármacos para la inducción de la actividad enzimática - Anticonceptivos de urgencia}

Los fármacos inductores de la actividad enzimática pueden afectar la eficacia contraceptiva de los métodos de anticonceptión de urgencia basados en levonorgestrel. En el caso del ulipristal, este método está contraindicado en mujeres que toman estos fármacos.

En este sentido, se debería recomendar a las mujeres que solicitan la anticonceptión de urgencia y están tomando un fármaco inductor de la actividad enzimática (o durante los 28 días porteriores a su interrupción) el uso de un DIU de cobre. Las mujeres que no desean usar este método o tienen alguna contraindicación para su uso, deben tomar $3 \mathrm{mg}$ de levonorgestrel en una única dosis, tan pronto como sea posible en las 120 horas posteriores a la relación coital sin protección.

Los fármacos que aumentan el pH gástrico (como los antiácidos, antagonistas de los receptores $\mathrm{H} 2$, o los inhibidores de la bomba de protones) reducen la absorción y eficacia del ulipristal utilizado como anticonceptivo de urgencia (132), y por lo tanto este método no debe recomendarse a mujeres que tomen este tipo de fármaco y solicitan un anticonceptivo de urgencia.

\subsection{Antibióticos que no alteran la actividad enzimática}

La mayoría de antibióticos, salvo algunos como la rifampicina y la rifabutina, no afectan la eficacia contraceptiva de los anticonceptivos hormonales, y por lo tanto no sugieren la necesidad de utilizar medidas de precaución adicionales.

El único aspecto a tener en cuenta sería en el caso que la toma del antibiótico provocara vómitos o diarreas, situación que podría afectar la absorción del anticonceptivo y por tanto requeriría medidas adicionales.

\subsubsection{Tratamientos que aumentan la concentración de los anticonceptivos hormonales}

El Anexo 3 recoge aquellos fármacos que aumentan la concentración de las hormonas anticonceptivas. Se desconoce el efecto clínico del aumento de la concentración de estinilestradiol o de la progesterona, aunque podrían aumentar los efectos adversos, pero en cualquier caso no afectaría su eficacia contraceptiva.

\subsubsection{Directrices ante posibles interacciones medicamentosas y los anticonceptivos hormonales}

El documento de recomendaciones de la FSRH (130), recoge las siguientes recomendaciones específicas a tener en cuenta ante una posible interacción medicamentosa y un anticonceptivo hormonal:

- Las mujeres que toman algún fármaco inductor de la actividad enzimática deben escoger algún método anticonceptivo que no esté afectado por la acción de estos fármacos (por ejemplo, inyectables de sólo progestágeno o un dispositivo intrauterino de levonorgestrel o de cobre). 
- Las mujeres que toman un fármaco inductor de la actividad enzimática durante menos de dos meses y que no desean cambiar de método anticonceptivo combinado por uno no afectado por la acción de estos fármacos, deben optar por un anticonceptivo oral con una dosis mínima de $30 \mu \mathrm{g}$ de etinilestradiol, un parche o un anillo con medidas anticonceptivas adicionales (preservativo). Esta medida debe mantenerse mientras dure el tratamiento con el fármaco inductor, y hasta 28 días después de su interrupción.

- Las mujeres que toman un fármaco inductor de la actividad enzimática durante un periodo superior a los dos meses y que no desean cambiar de método anticonceptivo combinado por uno no afectado por la acción de estos fármacos, deben optar por un anticonceptivo oral con una dosis incrementada hasta los $50 \mu \mathrm{g}$ de etinilestradiol. Esta medida debe mantenerse mientras dure el tratamiento con el fármaco inductor, y hasta 28 días después de su interrupción.

- Las mujeres que reciben un tratamiento con rifampicina o rifabutina, deben cambiar el método anticonceptivo por uno que no esté afectado por la acción de estos fármacos.

- Las mujeres que han tomado un fármaco inductor de la actividad enzimática durante menos de dos meses y que no desean cambiar el método anticonceptivo basado en la píldora o el implante de sólo progestágeno por uno no afectado por la acción de estos fármacos, deben optar por tomar precauciones anticonceptivas adicionales (preservativo). Esta medida debe mantenerse mientras dure el tratamiento con el fármaco inductor, y hasta 28 días después de su interrupción.

- Se debe aconsejar el uso de un DIU de cobre ó $3 \mathrm{mg}$ de levonorgestrel en una única dosis (tan pronto como sea posible, o al menos en las 120 horas posteriores a la relación coital sin protección) a las mujeres que solicitan un anticonceptivo de urgencia y están tomando un fármaco inductor de la actividad enzimática (o durante los 28 días porteriores a su interrupción).

- $\quad$ No debe usarse el ulipistral como método anticonceptivo de urgencia en aquellas mujeres que están tomando un fármaco inductor de la actividad enzimática (o durante los 28 días porteriores a su interrupción).

- $\quad$ No debe usarse el ulipistral como método anticonceptivo de urgencia en aquellas mujeres que están tomando un fármaco que aumente el $\mathrm{pH}$ gástrico (como los antiácidos, antagonistas de los receptores $\mathrm{H} 2$, o los inhibidores de la bomba de protones).

- No deben aconsejarse precauciones anticonceptivas adicionales a aquellas mujeres que están tomado un antibiótico (excepto en el caso de la rifampicina y la rifabutina, que son inductores de la actividad enzimática). 


\section{De la evidencia a la recomendación}

\section{Resumen de la evidencia}

Siempre debe tenerse en cuenta la posibilidad de una interacción medicamentosa en el momento de prescribir un anticonceptivo hormonal (incluso en anticoncepción de emergencia), o en el momento de prescribir un tratamiento a una mujer que está tomando un anticonceptivo hormonal (130).

Se debe advertir a las mujeres que están utilizando un anticonceptivo hormonal que ciertos medicamentos pueden alterar la eficacia del anticonceptivo y, por lo tanto, que puede ser necesario tomar alguna medida anticonceptiva adicional. La duración del tratamiento concomitante, y la causa por la que se debe prescribir este tratamiento, pueden ser aspectos determinantes en el momento de decidir qué anticonceptivo administrar.

Los documentos de criterios médicos de elegibilidad para el uso de anticonceptivos incluyen un apartado en los que se contemplan las principales interacciones medicamentosas y aquellas situaciones en las que un embarazo aumenta el riesgo de las mujeres con determinados problemas de salud (3-5).

\section{Calidad de la evidencia}

La calidad global es baja.

Toda la información recogida en esta pregunta clínica se basa en descripciones de casos, series de casos muy pequeñas, o alertas de fármacovigilancia, además de en recomendaciones elaboradas por Sociedades Científicas basadas en el criterio clínico. No se han elaborado tablas de evidencia GRADE para clasificar la calidad de la evidencia en esta pregunta.

\section{Valores y preferencias de las usuarias}

No se ha considerado este aspecto en esta pregunta clínica.

\section{Beneficios y riesgos/carga de las intervenciones}

No se ha considerado este aspecto en esta pregunta clínica.

\section{Balance beneficio/riesgo}

La aparición de una interacción medicamentosa puede tener una repercusión en la eficacia de un anticonceptivo hormonal, por lo que las recomendaciones en este sentido se basan en aquellas interacciones documentadas y en el criterio clínico.

\section{Uso de recursos y costes}

No se ha considerado este aspecto en esta pregunta clínica. 


\section{Justificación}

Las recomendaciones de este apartado se dirigen a identificar situaciones en las que pueda aparecer una interacción medicamentosa con el anticonceptivo hormonal. Las recomendaciones sobre anticoncepción e interacciones medicamentosas recogidas en los diferentes documentos de criterios médicos de elegibilidad para el uso de anticonceptivos se basan en los casos documentados de interacciones y en el criterio clínico, por lo que las recomendaciones en este apartado se clasifican como de buena práctica clínica.

\section{Recomendaciones}

\begin{tabular}{|l|l|}
\hline $\begin{array}{l}\text { Buena } \\
\text { práctica } \\
\text { clínica }\end{array}$ & $\begin{array}{l}\text { Si una mujer desea iniciar un tratamiento con anticonceptivos hormonales, } \\
\text { se debe recopilar información sobre los tratamientos que está tomando o ha } \\
\text { tomado recientemente. }\end{array}$ \\
\hline $\begin{array}{l}\text { Buena } \\
\text { práctica } \\
\text { clínica }\end{array}$ & $\begin{array}{l}\text { Se debe advertir a la mujer que está utilizando un anticonceptivo hormonal } \\
\text { que ciertos medicamentos pueden alterar la eficacia del anticonceptivo y } \\
\text { que debe consultar a un médico antes de iniciar cualquier tratamiento me- } \\
\text { dicamentoso. }\end{array}$ \\
\hline $\begin{array}{l}\text { Buena } \\
\text { práctica } \\
\text { clínica }\end{array}$ & $\begin{array}{l}\text { Cuando se realice con la mujer la elección del anticonceptivo debe consi- } \\
\text { derarse la duración de un posible tratamiento concomitante, la causa por } \\
\text { la que se debe prescribir este tratamiento, y aquellas situaciones en las que } \\
\text { puede ser necesario tomar alguna medida contraceptiva adicional. }\end{array}$ \\
\hline $\begin{array}{l}\text { Buena } \\
\text { práctica } \\
\text { clínica }\end{array}$ & $\begin{array}{l}\text { En la elección de un anticonceptivo ante una posible interacción medica- } \\
\text { mentosa se sugiere consultar cualquiera de los documentos disponibles para } \\
\text { determinar los criterios médicos de elegibilidad para el uso de anticoncep- } \\
\text { tivos. }\end{array}$ \\
\hline
\end{tabular}

\subsection{Manejo clínico de la anticoncepción intrauterina}

\subsubsection{Profilaxis antibiótica en la inserción de un DIU}

Se ha identificado una revisión sistemática Cochrane ((155) fecha de búsqueda actualizada en febrero de 2012), cuyos resultados se han publicado por duplicado (156), sobre el impacto del uso de la profilaxis antibiótica antes de la inserción de un DIU para reducir las complicaciones relacionadas con el dispositivo (principalmente Enfermedad Pélvica Inflamatoria -EPI). Otra revisión narrativa (87) sobre el riesgo de infección asociado al DIU se basó en los resultados de una revisión sistemática (155) para discutir la necesidad de realizar una profilaxis antibiótica en la inserción de un DIU. Esta revisión sistemática incluyó 4 ECA comparados con placebo (158 - 161), para los que se realizó un estudio piloto en 2 de ellos y de los cuales se analizaron sus resultados $(158,161)$.

Ladipo 1991 incluyó a 1485 mujeres nigerianas que solicitaron un DIU en un hospital universitario. Las mujeres debían tener entre 20 y 44 años y con ciclos menstruales regulares (a las que se realizó un cribado de gonorrea e infección por clamidias), y se excluyeron 
casos de embarazos ectópicos, embarazos dentro de los 42 días, a mujeres con enfermedades médicas, que habían usado un antibiótico en los últimos 14 días (159). Se aleatorizó a las participantes a recibir $200 \mathrm{mg}$ de doxiciclina oral 1 hora antes de la inserción o un placebo, para valorar la tasa de EIP y las visitas imprevistas al hospital.

Sinei 1990 incluyó a 1813 mujeres que solicitaron la inserción de un DIU en un centro de planificación familiar en Kenya (158). Los criterios de inclusión y exclusión, así como la intervención y los desenlaces de interés, fueron los mismos que en el estudio de Lapido 1991.

Walsh 1998 incluyó a 1867 mujeres que solicitaron la inserción de un DIU en 11 clínicas de California (EEUU) (161). La inclusión se realizó a criterio clínico de cada uno de los centros participantes en el estudio, pero en todos los centros se realizó un cribado de gonorrea e infección por clamidias. Se aleatorizó a las participantes para recibir $500 \mathrm{mg}$ de azitromicina oral una hora antes de la inserción o un placebo. Se evaluaron las razones de la extracción del DIU en los 90 días después de su inserción.

Por último Zorlu 1993 incluyó a 177 mujeres que acudieron a un centro de planificación familiar de Ankara (Turquía) solicitando la inserción de un DIU (160). Los motivos de exclusión del estudio fueron similares a los de los estudios desarrollados en África, pero no se realizó un cribado de gonorrea e infección por clamidias. Se aleatorizó a las participantes a recibir $200 \mathrm{mg}$ de doxiciclina oral 1 hora antes de la inserción o un placebo, que en el grupo de tratamiento se reforzó con dos dosis diarias adicionales de $200 \mathrm{mg}$ de doxiciclina. El desenlace principal de interés fue la tasa de EPI.

Un análisis combinado de los resultados de los estudios más rigurosos incluidos en una revisión sistemática $(155,158,159,161)$ no mostró una reducción del riesgo de la tasa de enfermedad pélvica inflamatoria al comparar la administración de profilaxis antibiótica con un placebo (3 ECA; 5520 mujeres; 26 eventos antibiótico vs 29 eventos placebo; OR 0,70; IC95\% $0,36$ a 1,38$)$.

De todos modos debe tenerse en cuenta la inclusión de estudios en poblaciones con una alta prevalencia de infecciones de transmisión sexual (158, 159), que quizás ofrecen resultados no extrapolables a población general. Un estudio mostró, por ejemplo, que la doxiciclina redujo de manera no significativa un tercio en el riesgo de EIP (1429 mujeres; 11 eventos antibiótico vs 16 eventos placebo; RR 0,69; IC95\% 0,32 a 1,47) (159), mientras que otro no mostró ninguna diferencia en este sentido (158). En cambio, en el estudio norteamericano (161), solamente se registró un caso de EIP en cada uno de los grupos (1.833 mujeres; 1 evento antibiótico vs 1 evento placebo; RR 1,13; IC95\% 0,70 a 1,81).

Calidad baja 
A este hecho debe sumarse la dificultad de estimar adecuadamente el riesgo de EIP asociada a la inserción del DIU por la complejidad del diagnóstico de esta infección y la variabilidad de definiciones existentes en la literatura, que pueden llevar a estimaciones que muestran diferencias 10 veces mayores entre ellas (87). El análisis de 12 estudios de la OMS, con datos correspondientes a 22.908 inserciones de DIU (seguimiento de 51.399 mujeres/año), mostró una incidencia de EIP en los primeros 20 días tras la inserción del dispositivo del 9,7 por 1000 mujeres/año, indicador que disminuyó drásticamente posteriormente tras este periodo hasta el 1,4 por 1000 mujeres/año (RR 6,3; IC95\% 3,4 a 11,6).

Una recomendación de la Faculty of Sexual and Reproductive Healthcare (162) descarta la necesidad de usar la profilaxis antibiótica antes de la inserción de un DIU incluso en los casos en que las mujeres tienen un mayor riesgo de endocarditis. Otras recomendaciones reconocen la ausencia de pruebas en la literatura que apoyen el uso de antibióticos profilácticos en la inserción de un DIU, pero consideran que en aquellas mujeres con una alta sospecha de infección, ésta debería confirmarse y tratar aquellas con un resultado positivo (163).

Por otro lado, aunque en mujeres con riesgo de endocarditis la inserción de un DIU puede estar contraindicada, el uso de antibióticos con la finalidad exclusiva de prevenir la endocarditis no estaría justificado si se decide su inserción $(164,165)$.

A pesar de estos datos y recomendaciones, los criterios de elegibilidad de la OMS aconsejan el uso de profilaxis antibiótica en mujeres con valvulopatía para prevenir la endocarditis (4), aunque no apoya con datos empíricos esta recomendación.

El análisis conjunto de los resultados de estos tres ensayos mostró que el uso de antibiótico comparado con placebo reduce mínimamente el número de visitas no programadas al hospital (3 ECA; 4917 mujeres; 320 eventos antibiótico vs 371 eventos placebo; OR 0,82; IC95\% 0,70 a 0,98$)$, pero no el número de mujeres que requirieron la extracción del dispositivo en los 90 días posteriores a la inserción del dispositivo (1 ECA; 2275 mujeres; 43 eventos antibiótico vs 41 eventos placebo; OR 1,05; IC95\% 0,68 a 1,63).

\subsubsection{Uso de misoprostol durante la inserción de un DIU}

Se ha identificado una revisión sistemática Cochrane ((166), fecha de búsqueda en septiembre de 2009) sobre el impacto de los tratamientos disponibles para el dolor asociado a la inserción de un DIU, que solamente incluye un estudio sobre el uso del misoprostol sobre el dolor percibido durante la inserción del DIU (167). Posteriormente a la revisión sistemática se han publicado seis estudios adicionales (168 - 173).

Los estudios disponibles varían en cuanto a la vía de administración del misoprostol y sus dosis, así como el momento de administración previo al procedimiento: 
El estudio incluido en la revisión de Curtis 2009 (167) comparó en 80 mujeres nulíparas el efecto de $400 \mu \mathrm{g}$ de misoprostol sublingual (juntamente con $100 \mathrm{mg}$ de diclofenaco) frente a una dosis de $100 \mathrm{mg}$ de diclofenaco, una hora antes de la inserción del DIU de cobre. La intervención de interés se cegó a los investigadores, pero no a las participantes en el estudio. Se evaluaron el dolor durante la inserción del dispositivo (mediante una escala visual analógica (EVA)), y la experiencia durante la inserción.

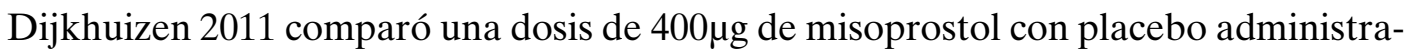
das 3 horas antes de la inserción de un DIU (indistintamente de cobre o liberador de levonorgestrel) en un ECA doble ciego, que incluyó a 199 mujeres nulíparas y multíparas en varios centros de Holanda. El principal desenlace de interés fue el fallo en la inserción del DIU, pero también se evaluaron las complicaciones derivadas de la inserción, el dolor, la dificultad experimentada durante la inserción y los efectos adversos. El estudio tuvo un importante número de pérdidas tras la aleatorización lo que puso en compromiso el cálculo del tamaño muestral inicial (168).

Edelman 2011 comparó la misma dosis de misoprostol que en los estudios anteriores con placebo en 40 mujeres nulíparas norteamericanas, administrado 90 minutos antes de la inserción de un DIU (indistintamente de cobre o liberador de levonorgestrel). El ensayo evaluó el dolor percibido como el desenlace principal, además de la facilidad de inserción, los efectos adversos y la retención del dispositivo 1 mes después de la inserción (169).

Scavuzzi 2013 comparó en un ensayo clínico doble ciego una dosis vaginal de misoprostol cuatro horas antes de la inserción de un DIU ( $400 \mathrm{mg}, 86$ mujeres que nunca habían estado embarazadas) frente a placebo (93 mujeres) (170). Lathrop 2013 también comparó

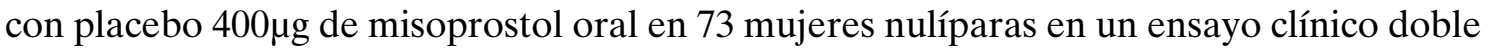
ciego (172). Finalmente, Espey 2014 realizó la misma comparación en un ensayo clínico doble ciego en el que se comparó el misoprostol oral ( 400 mcg; 42 mujeres nulíparas) con placebo (40 mujeres) (173).

Ibrahim 2013 comparó en un ensayo clínico simple ciego una dosis sublingual de misoprostol combinada con diclofenaco una hora antes de la inserción de un DIU (400 mg, 130 mujeres primíparas que habían dado a luz mediante cesárea electiva) frente a diclofenaco (100 mg; 125 mujeres) (171).

El estudio de Sääv 2007 no mostró diferencias significativas en la sensación del dolor o la satisfacción durante la inserción del DIU al comparar 400 Calidad $\mu \mathrm{g}$ de misoprostol administrados conjuntamente con $100 \mathrm{mg}$ de diclofenaco frente a $100 \mathrm{mg}$ de diclofenaco sólo (167). La puntuación media en la escala de dolor fue prácticamente la misma entre ambos grupos ( 7 puntos en el grupo diclofenaco frente 6,5 puntos en el grupo control), así como el número de mujeres que definieron la experiencia como desagradable o muy desagradable (1 ECA, 80 mujeres, 67 eventos; OR 2,19; IC 95\% 0,60 a 7,97). El número de mujeres que reportaron algún evento adverso (temblores, diarrea, nausea o vómito) fue similar entre el grupo de misoprostol y el control (1 ECA, 80 mujeres, 41 eventos; OR 1,76; IC 95\% 0,72 a 4,29). En el caso del temblor, se observó un mayor número de mujeres que manifestaron este efecto adverso entre las que recibieron misoprostol (14/40) que en el grupo control $(3 / 40)(\mathrm{P}=0,008)$. 
El ECA doble ciego de Dijkhuizen 2011 no mostró diferencias entre el misoprostol y el placebo en el fallo de la inserción del DIU (199 mujeres, 3 eventos; RR 1,9; IC 95\% 0,2 a 20,6), ni en las complicaciones durante la inserción (199 mujeres, problemas experimentados en el 20\% de las inserciones; RR 1,1; IC 95\% 0,7 a 2,0) (168). Tampoco se observaron diferencias entre los grupos en el dolor o la dificultad en la inserción percibidos, pero en cambio los efectos adversos fueron mayores entre el grupo de mujeres que recibió misoprostol (199 mujeres; 56\% frente a 42\%; RR 1,3; IC 95\% $1,0$ a 1,7$)$.

Edelman 2011 mostró resultados similares ya que las mujeres que recibieron misoprostol registraron puntuaciones similares de dolor en el momento de la inserción que las que recibieron placebo (puntuación en una EVA en el grupo misoprostol: $65 \mathrm{~mm}$ (DE $21 \mathrm{~mm}$ ) versus EVA en el grupo placebo: $55 \mathrm{~mm}$ (DE $21 \mathrm{~mm}$ ); $\mathrm{P}=0,83$ ) (169). Las mujeres del grupo misoprostol experimentaron náuseas en más ocasiones que las mujeres que recibieron el placebo ( 35 mujeres, $29 \%$ versus $5 \%$; $\mathrm{P}=0,05)$, así como temblores (35 mujeres, $47 \%$ versus $16 \% ; \mathrm{P}=0,04)$.

En el ensayo de Scavuzzi 2013, la dosis de $400 \mu \mathrm{g}$ de misoprostol mostró mejores resultados que el placebo en la dificultad de insertar el DIU (179 mujeres, RR 0,49; IC95\% 0,33 a 0,72; $\mathrm{P}=0,00005$ ), en el porcentajes de mujeres con dolor durante la inserción (RR 0,56; IC95\% 0,41 a 0,76; $\mathrm{P}=0,00008)$ o en aquellas que experimentaban una sensación desagradable (RR 0,49; IC95\% 0,35 a 0,68; $\mathrm{P}=0,00004$ ). No se observaron diferencias en las complicaciones durante la inserción del DIU (170).

En el ensayo de Ibrahim 2013, la dosis de $400 \mu \mathrm{g}$ de misoprostol combinada con diclofenaco mostró resultados similares al diclofenaco solo. Los fallos en la inseción del DIU fueron muy similares en ambos grupos (siete en el grupo de misoprostol, cinco en el control), y la facilidad de la inserción y la satisfacción de las usuarias fueron similares. Las mujeres que recibieron misoprostol describieron más dolor abdominal y náuseas (171).

En el ensayo de Lathrop 2013 los profesionales sanitarios tuvieron una percepción similar sobre la facilidad de insertar el DIU entre las mujeres que recibieron misoprostol o placebo. Las mujeres que recibieron misoprostol puntuaron en escalas analógicas (de o a 100 puntos) un dolor mayor tanto antes $(10,8$ frente a 2,1 puntos; $\mathrm{P}=0,003)$ como después de la inserción del DIU (46,5 frente a 35,1 puntos; $\mathrm{P}=0,04)$ (172).

Espey 2014 tampoco mostró diferencias entre la percepción de los profesionales sanitarios sobre la facilidad de insertar el DIU entre las mujeres que recibieron misoprostol o placebo, y las mujeres tuvieron una percepción similar de dolor (2,2 frente a 2,5 puntos en una escala de 0 a $10 ; \mathrm{P}=0,54)$ (173). 
Una publicación recoje los resultados de una encuenta en Estados Unidos en la que se les preguntó a profesionales sanitarios con experiencia en la inserción de un DIU sobre las posibles ventajas derivadas del uso del misoprostol en la inserción del dispositivo en mujeres nulíparas (174). Los resultados de la encuesta (con una repuesta del $86 \%$ de los 2200 profesionales encuestados electrónicamente) mostraron que el $42 \%$ de los profesionales usaban misoprostol antes de la inserción de un DIU. Entre las usuarias del misoprostol el 57\% opinó que el misoprostol facilitaba mucho el procedimiento.

La dosis más utilizada fue de $400 \mu \mathrm{g}(54 \%)$, la vía de administración más frecuente fue la vaginal $(40 \%)$ y la mitad de las usuarias administraban el misoprostol la noche antes de la inserción del DIU.

\section{De la evidencia a la recomendación}

\section{Resumen de la evidencia}

La administración rutinaria de profilaxis antibiótica antes de la inserción de un DIU ofrece poco beneficio en términos de evitar infecciones que afecten a los genitales internos (enfermedad pélvica inflamatoria), o en el número de visitas, incluso en poblaciones de alto riesgo (155).

El uso de misoprostol antes de la inserción de un DIU no ofrece ninguna ventaja en términos de dolor o satisfacción durante la inserción del dispoCalidad baja sitivo. Además, se asocia a un incremento de efectos adversos (167-173).

\section{Calidad de la evidencia}

La calidad global para el uso de profilaxis antibiótica en la inserción de un DIU es baja.

Aunque se dispone de 4 ensayos clínicos comparados con placebo para responder a esta pregunta clínica, los estudios se realizaron en poblaciones con una alta prevalencia de infecciones de transmisión sexual, o con un riesgo elevado de contraerlas. Por otro lado, el número de eventos observados en los estudios fue limitado afectando a la previsión de los resultados.

La calidad global para el uso de misoprostol antes de la inserción de un DIU es moderada.

Los resultados de los ensayos valorados para responder a esta pregunta clínica, han mostrado resultados consistentes aunque el número de eventos observados en los estudios fue limitado afectando a la previsión de los resultados. 


\section{Valores y preferencias de las usuarias}

Relacionado con el uso de misoprostol durante la inserción de un DIU, uno de los ensayos valorados mostró una proporción similar de mujeres que definió la experiencia de la inserción como desagradable o muy desagradable al usar misoprostol o diclofenaco (167). Los otros dos ensayos mostraron una sensación de dolor autopercibida similar entre las mujeres que tomaron misoprostol y las que tomaron placebo $(168,169)$. Las usuarias del ensayo clínico de Espey 2014 estaban dispuestas a tolerar un dolor similar independientemente de si recibían misoprostol o placebo (4,9 frente a 5,7 puntos en una escala de 0 a $10 ; \mathrm{P}=0,18)(173)$.

\section{Beneficios y riesgos/carga de las intervenciones}

Aunque los estudios evaluados no apoyan el uso rutinario de profilaxis antibiótica para la inserción de un DIU, la nueva edición de los criterios de elegibilidad de la OMS contempla la posibilidad de usar profilaxis antibiótica en mujeres con valvulopatía para prevenir la endocarditis (4).

\section{Balance beneficio/riesgo}

Los estudios evaluados no recogieron información relacionada con posibles efectos adversos derivados de la administración de antibióticos antes de la inserción del DIU. El uso del DIU es seguro y solamente se han reportado algunos casos de molestias tras la inserción o sangrados leves. Aunque en mujeres con riesgo de endocarditis la inserción de un DIU puede estar contraindicada, el uso de antibióticos con la finalidad exclusiva de prevenir la endocarditis no estaría justificado si se decide su inserción $(164,165)$.

Es necesario tener en cuenta que el uso de misoprostol no ofrece un beneficio en la inserción de un DIU en términos de reducción del dolor, aunque en todos los estudios valorados se ha mostrado un aumento de los efectos adversos. Incluso en el ensayo que comparó el misoprostol frente a diclofenaco (167), aunque el número de efectos adversos en general no fue diferente entre los grupos comparados, un mayor número de las mujeres que recibieron misoprostol mostraron temblor en mayor proporción que su comparador. Por su parte, los dos ensayos controlados con placebo $(168,169)$ mostraron que los efectos adversos fueron mayores entre el grupo de mujeres que recibió misoprostol.

\section{Uso de recursos y costes}

No existen evaluaciones económicas sobre el uso de la profilaxis antibiótica en la inserción de un DIU, aunque los autores de la revisión sistemática valorada cuestionan el coste beneficio del uso rutinario de este procedimiento (155).

\section{Justificación}

La administración de profilaxis antibiótica antes de la inserción de un DIU ha mostrado un beneficio mínimo, a pesar de la ventaja marginal en la reducción del número de visitas 
al hospital imprevistas. La realización rutinaria de esta práctica no parece aportar ningún valor adicional a la práctica clínica. Debe tenerse en cuenta que la literatura disponible es de baja calidad, principalmente debido a la disponibilidad de estudios que se han llevado a cabo en poblaciones con una alta prevalencia de infecciones de transmisión sexual, o con un riesgo elevado de contraerlas. No obstante, la baja incidencia de infecciones en poblaciones de bajo riesgo hace difícil disponer de estimadores precisos sobre el efecto de esta intervención. Si bien la recomendación se refiere a la administración rutinaria de profilaxis antibiótica en la inserción de un DIU, debe tenerse en cuenta que los criterios de elegibilidad de la OMS contemplan la posibilidad de usarlos en mujeres con valvulopatía para prevenir la endocarditis (4).

Por otro lado, a pesar de las diferencias entre los estudios disponibles, el uso de misoprostol antes de la inserción de un DIU no ha mostrado ninguna ventaja en términos de dolor o satisfacción durante la inserción del dispositivo, asociándose a un incremento de efectos adversos. Alguno de los estudios evaluados además ha mostrado la insatisfacción de las usuarias con el procedimiento.

\section{Recomendaciones}

\begin{tabular}{|l|l|}
\hline Fuerte & $\begin{array}{l}\text { No se recomienda administrar profilaxis antibiótica en la inserción de un } \\
\text { DIU. }\end{array}$ \\
\hline Fuerte & $\begin{array}{l}\text { No se recomienda administrar misoprostol de manera rutinaria antes de la } \\
\text { inserción de un DIU con la finalidad de facilitar este procedimiento. }\end{array}$ \\
\hline
\end{tabular}





\section{Situaciones específicas}

Preguntas a responder:

- ¿A qué edad puede considerarse la utilización de la anticoncepción hormonal en mujeres adolescentes?

- ¿Es adecuada la utilización de un DIU en las mujeres adolescentes?

- ¿Hasta qué edad es necesaria la anticoncepción hormonal?

- ¿Se debe cambiar el DIU de cobre o LNG insertado después de los cuarenta años?

- ¿Deben realizarse consideraciones especiales en usuarias de anticonceptivos hormonales o intrauterinos con sobrepeso u obesidad?

- ¿En qué momento se puede iniciar un tratamiento anticonceptivo hormonal o intrauterino tras el parto?

\subsection{Usuarias adolescentes}

\subsubsection{Edad de inicio de la anticoncepción hormonal}

Los diferentes criterios de elegibilidad disponibles para el uso de anticonceptivos $(3,4)$ o documentos con directrices para el uso de anticonceptivos en adolescentes (175), establecen el inicio de la anticoncepción hormonal en la menarquia. Los diferentes criterios de elegibilidad identifican la menarquia como el momento en el que se puede iniciar la anticoncepción hormonal sin restricciones (categoría 1) o de una manera en la que los beneficios del método son mayores que sus posibles riesgos (categoría 2). La siguiente tabla muestra las indicaciones de los anticonceptivos hormonales en las diferentes propuestas siguiendo el inicio de la menstruación como criterio: 


\begin{tabular}{|c|c|c|c|c|c|c|c|}
\hline & Píldora & Parche & Anillo & $\begin{array}{c}\text { Sólo } \\
\text { gestágeno }\end{array}$ & Inyectable & Implante & DIU de LNG \\
\hline $\begin{array}{c}\text { CDC } \\
2013 \\
(3)\end{array}$ & \multicolumn{3}{|c|}{$\begin{array}{l}\text { Entre menarquia y }<40 \text { años } \\
1\end{array}$} & $\begin{array}{c}\text { Entre } \\
\text { menarquia y } \\
45 \text { años } \\
1\end{array}$ & $\begin{array}{c}\text { Entre menarquia y } \\
<18 \text { años } \\
2 \\
18 \text { a } 45 \text { años } \\
1\end{array}$ & $\begin{array}{c}\text { Entre } \\
\text { menarquia y } \\
45 \text { años } \\
1\end{array}$ & $\begin{array}{c}\text { Entre } \\
\text { menarquia y } \\
<18 \text { años } \\
2\end{array}$ \\
\hline $\begin{array}{c}\text { WHO } \\
2015 \\
(4)\end{array}$ & \multicolumn{3}{|c|}{$\begin{array}{l}\text { Entre menarquia y }<40 \text { años } \\
\qquad 1^{1}\end{array}$} & $\begin{array}{c}\text { Entre } \\
\text { menarquia y } \\
45 \text { años } \\
1\end{array}$ & $\begin{array}{c}\text { Inyectable } \\
\text { combinado } \\
\text { Entre menarquia y } \\
<40 \text { años } \\
1 \\
\text { Inyectable DMPA } \\
\text { Entre menarquia y } \\
<18 \text { años } \\
2 \\
18 \text { a } 45 \text { años } \\
1\end{array}$ & $\begin{array}{c}\text { Entre } \\
\text { menarquia y } \\
45 \text { años } \\
1\end{array}$ & $\begin{array}{c}\text { Entre } \\
\text { menarquia y } \\
<20 \text { años } \\
2^{2}\end{array}$ \\
\hline $\begin{array}{c}\text { UK } \\
\text { MEC } \\
2009 \\
(5)\end{array}$ & \multicolumn{3}{|c|}{$\begin{array}{l}\text { Entre menarquia y }<40 \text { años } \\
1\end{array}$} & $\begin{array}{c}\text { Entre } \\
\text { menarquia y } \\
45 \text { años } \\
1\end{array}$ & $\begin{array}{c}\text { Inyectable DMPA } \\
\text { Entre menarquia y } \\
<18 \text { años } \\
2 \\
18 \text { a } 45 \text { años } \\
1\end{array}$ & $\begin{array}{c}\text { Entre } \\
\text { menarquia y } \\
45 \text { años } \\
1\end{array}$ & $\begin{array}{c}\text { Entre } \\
\text { menarquia y } \\
<20 \text { años } \\
2^{2}\end{array}$ \\
\hline
\end{tabular}

1. Advierte del impacto de los anticonceptivos con $20 \mu \mathrm{g}$ EE sobre la densidad mineral ósea, que desaparece a dosis más altas.

2. El uso de DIU en esta edad comporta una serie de consideraciones sobre la posibilidad de expulsión por la nuliparidad, y adicionalmente debe tenerse en cuenta el riesgo de ITS por el comportamiento sexual durante esta etapa de la vida.

Las particularidades de esta franja etaria, hacen que documentos como los criterios de elegibilidad de los CDC estadounidense identifiquen a las adolescentes como un grupo de usuarias de anticoncepción que podría beneficiarse de una mayor frecuencia de visitas en las que poder valorar la adherencia al método elegido, eventualidades relacionadas con su uso, dudas de las usuarias, valorar aspectos como el riesgo de ITS de las usuarias u otros más clínicos (3).

Por otro lado, se ha propuesto el método de inicio inmediato ('quick start' por referencia en inglés) como un abordaje del que podrían beneficiarse las usuarias adolescentes (176). El método de inicio inmediato pretende mejorar el cumplimiento con el método y prevenir embarazos en el periodo de espera para empezar la anticoncepción hormonal. Este enfoque propone el inicio del tratamiento el mismo día en el que se prescribe el método hormonal, habiendo excluido la posibilidad de embarazo. En el método estándar el comienzo del tratamiento se retrasaría hasta la aparición o momento avanzado de la regla, de modo que se mantenga la regularidad del ciclo y se evita la exposición hormonal en un eventual embarazo. 
Un estudio retrospectivo en 193 adolescentes con una media de 16 años de edad, usuarias de anticonceptivos orales, comparó el cumplimiento con el método de inicio inmediato frente a un método habitual de inicio el domingo posterior al inicio del próximo ciclo (177). Aunque el método inmediato mostró un mejor cumplimiento a los tres meses de seguimiento $(27 \%$ frente a $56 \% ; \mathrm{P}=0,059)$, las diferencias habían desaparecido al año de seguimiento. La seguridad de ambos métodos fue similar.

Un estudio prospectivo permitió escoger el método de inicio a las mujeres que asistían a una clínica de planificación familiar para iniciar o retomar un método anticonceptivo hormonal (178). El estudio analizó los datos de 57 jóvenes que escogieron el método inmediato y de 156 que escogieron el método habitual. Aunque las participantes tuvieron una media de edad de 22 años y la publicación no ofrece datos adicionales sobre las usuarias adolescentes, el método inmediato fue uno de los principales predictores de la continuidad entre el primer y segundo tratamiento anticonceptivo (OR 2,8; IC95\% 1,1 a 7,3).

Los investigadores de este estudio observacional diseñaron un ensayo clínico para comprobar la eficacia en términos de continuidad con el método y embarazos del método inmediato frente al habitual $(179,184)$, cuyos resultados en las 539 participantes adolescentes (con una media de 16 años de edad) se publicaron por separado (180). El método inmediato mostró una mayor probabilidad de continuidad entre el primer y segundo tratamiento anticonceptivo (OR 1,8; IC95\% 1,1 a 3,3), pero no mostró una mejor continuidad en el uso a los tres o seis meses de seguimiento, momento en el que solamente el $26 \%$ de las participantes seguían con el método.

La tasa de embarazos entre los dos métodos fue similar $(6,5 \%$ frente a 10,5\%; RR 0,60; IC95\% 0,33 a 1,06; P=0,08).

Otros ensayos clínicos incluidos en una revisión sistemática Cochrane que evaluó el impacto del método inmediato (181) no se han valorado ya que no ofrecían datos desagregados para las usuarias adolescentes (182-184), o comparaban dos estrategias de métodos inmediatos de anticoncepción (185).

\subsubsection{Utilización de DIU en las mujeres adolescentes}

Este apartado se basa en los resultados de una revisión sistemática de estudios observacionales sobre el uso del DIU en adolescentes (186) y los resultados del estudio CHOICE, una cohorte que evalúa un programa comunitario dirigido a promover el uso de anticonceptivos reversibles de acción prolongada (LARC por sus siglas en inglés) (17, 187).

Una revisión sistemática incluyó 13 estudios de cohortes o casos y controles (186). Los autores de la revisión advirtieron que la literatura disponible en este campo es insuficiente y en muchas ocasiones obsoleta. La siguiente tabla resume los estudios incluidos en esta revisión: 


\begin{tabular}{|c|c|c|c|c|c|c|}
\hline Estudio & Participantes & Tipo de DIU & Edad & Seguimiento & Desenlace & Comentario \\
\hline \multicolumn{7}{|c|}{ estudios de cohorte } \\
\hline $\begin{array}{l}\text { Jorgensen } \\
1973 \text { (188) }\end{array}$ & $\begin{array}{l}172 \\
\text { (82 con DIU) }\end{array}$ & $\begin{array}{l}\text { Lippes Loop A } \\
\text { Cooper T }\end{array}$ & $\begin{array}{c}11 \mathrm{a} \\
17\end{array}$ & 12 meses & $\begin{array}{l}\text { Uso } \\
\text { continuado } \\
\text { Expulsión }\end{array}$ & Post parto \\
\hline $\begin{array}{l}\text { Goldman } \\
1979 \text { (189) }\end{array}$ & 162 & $\begin{array}{l}\text { Lippes Loop A } \\
\text { Cooper } 7 \\
\text { Cooper T }\end{array}$ & $\begin{array}{c}13 a \\
18\end{array}$ & 12 meses & $\begin{array}{l}\text { Uso } \\
\text { continuado } \\
\text { Embarazo } \\
\text { Expulsión }\end{array}$ & $\begin{array}{l}\text { Nulíparas } \\
\text { post aborto }\end{array}$ \\
\hline $\begin{array}{l}\text { Goldman } \\
1980(190)\end{array}$ & $\begin{array}{l}160 \\
\text { (30 con DIU) }\end{array}$ & $\begin{array}{l}\text { Cooper } 7 \\
\text { Cooper T }\end{array}$ & $\begin{array}{c}14 a \\
18\end{array}$ & 24 meses & $\begin{array}{l}\text { Uso } \\
\text { continuado } \\
\text { Embarazo } \\
\text { Expulsión }\end{array}$ & Nulíparas \\
\hline $\begin{array}{l}\text { Díaz } 1993 \\
\text { (191) }\end{array}$ & 995 & T-Cu200B & $<20$ & 48 meses & $\begin{array}{l}\text { Uso } \\
\text { continuado } \\
\text { Embarazo } \\
\text { Expulsión }\end{array}$ & - \\
\hline \multicolumn{7}{|c|}{ estudios de casos y control } \\
\hline $\begin{array}{l}\text { Lane } 1975 \\
\text { (192) }\end{array}$ & 103 & $\begin{array}{l}\text { Loop C } \\
\text { Loop D } \\
\text { Experimental }\end{array}$ & $\begin{array}{c}13 a \\
19\end{array}$ & 9 meses & $\begin{array}{l}\text { Uso } \\
\text { continuado } \\
\text { Embarazo } \\
\text { Expulsión }\end{array}$ & $\begin{array}{l}\text { Nuligrávidas } \\
\text { (83\%) } \\
\text { Nulíparas } \\
(96 \%)\end{array}$ \\
\hline $\begin{array}{l}\text { Weiner } 1978 \\
\text { (193) }\end{array}$ & 243 & $\begin{array}{l}\text { Cooper } 7 \\
\text { Cooper T-200 }\end{array}$ & $\begin{array}{c}13 a \\
20\end{array}$ & 6 meses & $\begin{array}{l}\text { Uso } \\
\text { continuado } \\
\text { Embarazo } \\
\text { Expulsión }\end{array}$ & Nuligrávidas \\
\hline $\begin{array}{l}\text { Kulig } 1980 \\
(194)\end{array}$ & 120 & Cooper 7 & $\begin{array}{c}13 a \\
22\end{array}$ & 36 meses & $\begin{array}{l}\text { Uso } \\
\text { continuado } \\
\text { Embarazo } \\
\text { Expulsión }\end{array}$ & $\begin{array}{l}\text { Nulíparas } \\
(81 \%)\end{array}$ \\
\hline $\begin{array}{l}\text { Larsson } \\
1981 \text { (195) }\end{array}$ & 179 & Cooper 7 & $\begin{array}{c}15 a \\
19\end{array}$ & 24 meses & Embarazo & - \\
\hline
\end{tabular}

El estudio CHOICE es una cohorte prospectiva oportunista en EEUU, diseñada para comprobar si los LARC reducen el riesgo de embarazo no deseado. Las participantes en este programa comunitario recibían información sobre anticoncepción reversible, insistiendo en los beneficios de los LARC. El programa suministraba sin coste alguno el anticonceptivo que elegían las propias participantes y realizaba un seguimiento de hasta 3 años $(17,187)$. El objetivo principal del estudio fue el de comparar la tasa de fallo de los anticonceptivos LARC frente a la píldora, parche y anillo (PPA) o el inyectable de acetato de medroxiprogesterona (DMPA).

Los resultados para la cohorte completa (17) se detallan en la pregunta sobre la eficacia y seguridad de los anticonceptivos, aunque éstos ya mosCalidad traron que el riesgo de embarazo no deseado en las mujeres menores de 21 baja años que eligieron la PPA era el doble del de sus pares mayores (HR 1,83; IC95\% 1,25 a 2,69), mientras que la tasa de embarazo no deseado no varió entre las usuarias de LARC o DMPA en función de la edad. 
Estos resultados se han complementado con el análisis de las tasas de embarazo, nacimientos y abortos inducidos de 1404 participantes en el estudio CHOICE con una edad comprendida entre los 14 y 19 años (187). Casi la mitad de las participantes en el CHOICE de esta franja etaria tenía al menos un antecedente de embarazo no deseado (47,8\%), y el $18 \%$ de un aborto. La mayoría de las participantes eligieron un LARC como método anticonceptivo, siendo mucho más escogido por las adolescentes de entre 14 y 17 años que entre las que tenían 18 a 19 años (77,5\% frente a 68,4\%; $\mathrm{P}<0,001)$. El implante fue el método más escogido por las primeras $(50 \%)$ mientras que el segundo grupo escogió el DIU en una mayor proporción $(35,4 \%)$.

El programa se mostró eficaz en la población evaluada, puesto que en un periodo de 6 años la tasa anual de embarazo fue más baja (34 embarazos por cada 1.000 usuarias/año; IC95\% 25,7 a 44,1) comparada con la tasa del resto de población estadounidense de la misma edad (57,4 embarazos por cada 1.000 usuarias/año) o con la de la misma edad sexualmente activa (158,5 embarazos por cada 1000 usuarias/año).

El estudio describió diferencias muy relevantes en la tasa de fallo de los diferentes métodos anticonceptivos. El método con más fallos fue el parche (60,8 fallos por cada 1.000 usuarias/año), seguido de la píldora $(56,8$ por cada 1.000 usuarias/año), el anillo (51,8 por cada 1.000 usuarias/año). El DMPA inyectable (5,2 fallos por cada 1000 usuarias/año) y el DIU de levonorgestrel (5,1 por cada 1000 usuarias/año) fueron los métodos con una menor tasa de fallo. Finalmente, no se registraron fallos entre las usuarias del DIU de cobre o el implante.

Los estudios incluidos en una revisión de estudios observacionales (186), con seguimientos entre los 6 y 48 meses, también mostraron que los embarazos eran infrecuentes (188-195). La tasa acumulada de embarazo varió del 2\% a los 6 meses de seguimiento (193) hasta el $11 \%$ a los 48 meses (191). Un estudio comparó la tasa de embarazo entre el DIU (3\%) y la píldora (0\%) (190).

Las adolescentes muestran una mejor continuidad con el uso de los métodos anticonceptivos de acción prolongada, que con los métodos de acción más breve o que requieren un uso diario o rutinario.

Los datos de continuidad en las participantes en la cohorte CHOICE mostraron que el porcentaje de uso a los 12 meses de seguimiento de los LARC era muy aceptable entre las adolescentes y las mujeres jóvenes (196). Se analizaron los datos de continuidad de uso a los 12 meses de seguimiento de las 7472 participantes en el programa comparando los resultados para las franjas etarias 14 a 19 años, 20 a 25 y 26 o mayores. 
La continuidad de uso de los anticonceptivos LARC entre las adolescentes fue ligeramente menor que entre las participantes de más edad, aunque la diferencia no alcanzó la significación estadística ( $81 \%$ frente a $86 \%)$. En contraste, el porcentaje de adolescentes que dejaba de usar otro tipo de anticonceptivos era estadísticamente mayor que entre las mujeres mayores de 25 años (53\% frente a $44 \%$; HR 1,32; IC95\% 1,02 a 1,73). Además, pese a mostrar diferencias pequeñas, las adolescentes mostraban una menor satisfacción que las usuarias de más edad con los anticonceptivos diferentes a LARC (42\% frente a 51\%; RR 0,80; IC95\% 0,65 a 0,98), diferencia que no se apreciaba con los métodos LARC (75\% frente a 83\%; RR 0,94; IC95\% 0,88 a 1,01).

En los siete estudios que aportaron datos sobre la continuidad del uso del anticonceptivo (188 - 194), se observó que la continuación era alta durante el primer año y disminuía con el tiempo. Durante el primer año entre el $48 \%$ (189) y el 88\% (188) de las participantes de los estudios seguían con el dispositivo, a los 24 meses entre el 49\% (194) y el 73\% (191), a los 36 meses entre el 39\% (194) y el 45\% (191), y finalmente un 31\% de las participantes en el estudio de Díaz 1993 continuaban con el DIU a los 48 meses (191).

Estudios posteriores han mostrado resultados similares. En una cohorte neozelandesa en 179 adolescentes (entre los 11 y 19 años) que usaron un DIU de levonorgestrel, la tasa de continuación del tratamiento al año de seguimiento fue del $85 \%$. De todos modos, en este estudio solamente el $14 \%$ de las participantes usaron el DIU con una finalidad exclusivamente anticonceptiva, con un número considerable de participantes que usaron el DIU con finalidades anticonceptivas y también como tratamiento de la menorragia (16\%) o por otras causas $(25 \%)$. El resto de participantes $(55 \%)$ no usó el DIU con finalidades anticonceptivas (197).

En los estudios que se comparó el uso de DIU con el de otros dispositivos $(188,190)$, las tasas de cumplimiento fueron similares. En un ECA se observó que las mujeres menores de 25 años interrumpieron el uso del dispositivo de levorgestrel en una mayor proporción que mujeres de mayor edad (198), mientras que en otro se observó que las principales razones para la interrupción del uso del DIU-Cu entre las participantes adolescentes fueron el dolor y el sangrado (199).

El sangrado es una de las principales razones de discontinuación del DIU. En los estudios recogidos en la revisión de Deans 2009 (186), las participantes adolescentes que habían abandonado el DIU por esta causa en el estudio de Díaz 1993 (17\%) fue mayor que las mujeres adultas que discontinuaron por la misma causa (9\%) (191). En el estudio de Larsson 1981 los abandonos por sangrado o dolor a los dos años de seguimiento, fueron similares entre las mujeres de diferentes edades (195). Por último en el estudio de Goldman 1980 hubo un mayor número de adolescentes con DIU que reportaron problemas de sangrado $(27 \%)$ que las adolescentes que tomaban otros anticonceptivos (11\%) (190). 
El estudio CHOICE ha publicado los datos relacionados con la tasa de sangrado y manchado de las usuarias de DIU e implante a los tres y seis meses de uso, y su posible asociación con la satisfacción con el método (200). No obstante los resultados se han publicado para todas las participantes en el estudio, sin que se disponga de los datos desagregados por franja etaria. Además, las participantes de menos de 20 años solamente fueron el $20 \%$ de la muestra.

Mientras que más del $65 \%$ de usuarias del DIU de levonorgestrel o del implante no reportaron modificaciones relevantes en su patrón de manchado, el $63 \%$ de las usuarias del DIU de cobre describieron un aumento del manchado menstrual. Un número considerable de usuarias del implante y el DIU de levonorgestrel describieron un patrón de sangrado menor que al inicio del estudio (58\% y $67 \%$, respectivamente), hecho que solamente reportaron el $8 \%$ de las usuarias de DIU de cobre.

Aunque la satisfacción con los anticonceptivos LARC fue muy buena a los seis meses de uso, fue menos probable que las usuarias reportaran estar satisfechas con el método si habían experimentado un aumento del sangrado menstrual (RR 0,78; IC95\% 0,72 a 0,85), mayores sangrados (RR 0,83; IC95\% 0,76 a 0,92$)$, o si había aumentado su frecuencia de sangrado $(0,73$; IC95\% 0,67 a 0,80$)$.

La tasa de expulsiones del dispositivo fue muy variable (188-194, 197), entre el $5 \%$ y el $22 \%$, tasa que se relacionó de manera inversa (a más edad, menos expulsiones) en dos estudios $(191,195)$. Esta relación entre la edad y la tasa de expulsiones se observó en un estudio de casos y controles (201) que mostró una mayor tasa de expulsiones entre las participantes que anteriormente había experimentado una expulsión y entre las mujeres más jóvenes. No obstantes la variable independiente en este análisis se refirió a mujeres menores de 35 años. El estudio solamente incluyó un 2\% de participantes entre los 18 y 24 años y en este subgrupo no se observaron diferencias significativas (20 participantes; OR 0,5; IC95\% 0,17 a 1,42).

En un análisis secundario de los resultados del estudio CHOICE, se valoró si la edad y la nuliparidad se asociaban a la tasa de expulsión de los DIU (202). Durante los tres años del estudio se registraron 432 expulsiones en las 5403 usuarias (el 78\% de la muestra usó un DIU de levonorgestrel, y el $22 \%$ uno de cobre). La tasa acumulada de 10,2 expulsiones por cada 100 usuarias/año no varió dependiendo del dispositivo (DIU LNG 10,1 frente a DIU Cu 10,7; P=0,99). La tasa acumulada de expulsión fue significativamente mayor entre las usuarias de 14 a 19 años que en las usuarias mayores (18,8 frente a 9,3 expulsiones por 100 usuarias/año; $\mathrm{P}<0,001)$. Un análisis ajustado a los factores de confusión confirmó el mayor riesgo de expulsión en las usuarias adolescentes independientemente del tipo de dispositivo (HR DIU LNG 2,26; IC95\% 1,68 a 3,06; HR DIU Cu 3,06; IC95\% 1,75 a $5,33)$. 
Uno de los puntos de interés sobre el uso del DIU en adolescente es el riesgo de enfermedad pélvica inflamatoria (EPI). En las mujeres de cualquier edad, el riesgo de EIP es mayor en los tres primeros meses tras la inserción del DIU (9,7 por 1000 mujeres/año), y se reduce posteriormente (1,4 por 1000 mujeres/año), una tasa similar a las mujeres que no usan DIU y relacionado más a infecciones de transmisión sexual que al uso del dispositivo (102). La definición de EIP está recogida de manera muy heterogénea en la literatura focalizada en población adolescente (186), aunque se ha destacado que incluso en presencia de infección, la tasa de EIP es baja, oscilando entre el $0 \%$ y el $5 \%$ (155 - 157).

En algunos países como los EEUU, las adolescentes presentan hasta 39\% de los casos de clamidia y el $34 \%$ de los casos de gonorrea del total registrados ( $67 \%$ y $56 \%$ si se amplía la franja de edad hasta los 24 años de edad) (203), lo que justifica la recomendación de realizar un cribado antes de la inserción de un DIU (3).

En nuestro entorno se ha observado una tendencia ascendente en el periodo 2007 a 2012 de diagnósticos de Chlamydia trachomatis registrados por los laboratorios que notifican al Sistema de Información Microbiológica del Instituto de Salud Carlos III (101 casos en 2007 y 799 en 2012) (204). Aunque la distribución de los diagnósticos es similar entre hombres y mujeres, éstos afectan principalmente a la población más joven, y en la franja de los 15 a 19 años afectan en una mayor proporción a las mujeres. En la franja de los 10 a 14 años los únicos casos registrados son en mujeres. Datos de prevalencia locales han mostrado una prevalencia de Chlamydia trachomatis durante el año 2012 en la franja de edad entre los 16 y los 24 años del 8,5\% (con 43 casos positivos). La prevalencia en mujeres fue mayor $(9,1 \%)$ que en los hombres $(2,2 \%)$, sin que la diferencia fuera estadísticamente significativa (205).

Como se ha comentado en la pregunta clínica sobre los controles necesarios en anticoncepción, en mujeres con un riesgo bajo de ITS sería suficiente que durante la exploración previa al inicio de un método anticonceptivo se incorporen en la anamnesis aspectos relacionados con los hábitos sexuales de las futuras usuarias con el objetivo de descartar el riesgo de ITS, sin que sea necesario realizar serologías de manera rutinaria. En caso de detectar signos o síntomas de alguna ITS, es necesario realizar una serología y esperar su resultado, y no insertar un DIU hasta que se complete el tratamiento en caso de un resultado positivo (157). 
Las tasas de infertilidad no son superiores en usuarias de DIU al compararlas con las usuarias de otros métodos anticonceptivos. En un estudio de cohorte, entre 109 mujeres noruegas que interrumpieron el DIU por deseo gestacional, el 93,6\% se quedaron embarazadas sin observarse diferencias entre los embarazos intra o extrauterinos (206). Aunque solamente el 12\% de estas participantes eran adolescentes (14/109) un análisis de los resultados mostró que la tasa de embarazo no dependía de la edad en la que la mujer interrumpía el tratamiento.

En los criterios de elegibilidad, la franja etaria comprendida entre la menarquia hasta los 20 años los dispositivos intrauterinos reciben la categoría 2. Esta indicación se acompaña de la advertencia de que el uso de DIU en esta edad comporta una serie de consideraciones sobre la posibilidad de expulsión por la nuliparidad, y adicionalmente debe tenerse en cuenta el riesgo de ITS por el comportamiento sexual durante esta etapa de la vida.

No obstante, debe tenerse en cuenta que ante una cervicitis purulenta o infección por Chlamydia o gonococo los DIU están contraindicados (categoría 4), pero mantienen la categoría 2 para su continuación en el uso o ante la presencia de otras ITS, salvo en casos de riesgo individual de ITS alto en las que se les otorga la categoría 3. Los criterios de elegibilidad también remarcan que los DIU no protegen contra las ITS, por lo que ante el riesgo de exposición a una ITS se debe aconsejar el uso del preservativo (4).

\section{De la evidencia a la recomendación}

\section{Resumen de la evidencia}

Los principales criterios de elegibilidad para el uso de anticonceptivos establecen el inicio de la anticoncepción hormonal en la menarquia (3-5).

El impacto del inicio inmediato de la anticoncepción sobre la continuidad con el método elegido y la tasa de embarazos no deseados en adolescentes, es limitado. El método inmediato ha mostrado una mejora en la continuidad a muy corto plazo, que no se mantiene a medio o largo plazo $(177,180)$, sin mayor impacto sobre la tasa de embarazos no deseados (180).

La edad de las usuarias tiene un impacto relevante en la tasa de fallo de determinados anticonceptivos. Mientras que la tasa de fallo no varía en Calidad baja función de la edad en las usuarias de anticonceptivos reversibles de acción prolongada (LARC) o el inyectable de DMPA, las usuarias menores de 21 años han mostrado una tasa de fallo mucho mayor que sus pares mayores con otros métodos como la píldora, parche transdérmico o anillo (17). 
Un estudio que ha evaluado un programa de consejo anticonceptivo en el que se promocionaba el uso de anticonceptivos LARC en adolescentes, mostró diferencias muy importantes en las tasas de fallo dependiendo del método. Mientras que el parche o la píldora tenían tasas de fallo considerables (60,8 y 56,8 fallos por cada 1000 usuarias/año respectivamente), el DIU de levonorgestrel mostró una tasa de fallo de 5,1 fallos por cada 1000 usuarias/año. Métodos como el DIU de cobre o el implante no mostraron fallos (187).

Datos de este mismo estudio han mostrado que entre las usuarias adolescentes la continuidad de uso también es diferente al de las usuarias de más edad, dependiendo del tipo de anticonceptivo. Mientras que la continuidad en el uso entre las adolescentes de los anticonceptivos LARC era ligeramente menor que las usuarias de más edad, la proporción de adolescentes que abandonaba el uso de otro tipo de anticonceptivos era mucho mayor que las usuarias de más edad. La satisfacción con los anticonceptivos diferentes a los LARC era mucho menor entre las adolescentes que entre las usuarias mayores (196).

Las alteraciones del patrón de sangrado entre las usuarias de DIU de cobre son frecuentes, y éstas pueden determinar la satisfacción con el método anticonceptivo escogido (200).

La tasa de expulsión de los DIU se asocia con la edad de las usuarias. Las usuarias adolescentes tienen más probabilidad de expulsar el dispositivo que las usuarias de más edad, independientemente de la paridad y el tipo

Calidad baja

Calidad baja

Calidad baja

Calidad baja de dispositivo (202).

\section{Calidad de la evidencia}

La calidad global es baja.

La calidad de la evidencia para el apartado sobre el inicio inmediato de la anticoncepción en usuarias adolescentes es baja. La evaluación se ha basado en los datos de dos estudios observacionales y un sub-análisis de un ensayo clínico. Uno de los estudios observacionales fue retrospectivo (177) y el otro incluyó a participantes con una edad mayor de la de interés para la pregunta clínica (178). Por otro lado, los datos de Edwards 2008 corresponden a un re-análisis de los datos correspondientes a las adolescentes participantes en un ensayo clínico. Además de las limitaciones relacionadas con el diseño del estudio, los datos no tienen el poder estadístico necesario para mostrar una diferencia clínicamente relevante (180).

La calidad de la evidencia para la eficacia y seguridad de los DIU, así como para el resto de desenlaces, en mujeres adolescentes se considera baja. Todos los estudios considerados son estudios observacionales, que en ocasiones evaluaron métodos obsoletos como se destaca en la revisión sistemática de Deans 2009 (186). Por otro lado se dispone de un gran estudio observacional que ha permitido valorar el impacto de los LARC. El estudio CHOICE recoge los resultados de un estudio de cohorte prospectivo con un buen 
diseño y con unos estimadores del efecto considerables. No obstante, teniendo en cuenta que la población de estudio puede no ser representativa de la de nuestro entorno, se ha mantenido la calidad de la evidencia como baja.

\section{Valores y preferencias de las usuarias}

En el momento de iniciar su participación en el estudio CHOICE las participantes completaron una encuesta en las que puntuaron la importancia de determinados atributos para la elección de un método anticonceptivo, y cuáles eran los atributos más importantes en el momento de optar por un método (109). Aunque los resultados publicados no ofrecen información desagregada para las diferentes franjas etarias, los atributos puntuados como más importantes por las 2.590 participantes en la encuesta fueron la eficacia, la seguridad, la asequibilidad del método, su duración, y la posibilidad de olvidar el método. Por otro lado, las mujeres que daban importancia a la duración del método y la posibilidad de olvidarlo eran más proclives a escoger un DIU. Del mismo modo, las mujeres que daban importancia a no sufrir alteraciones en el patrón de sangrado, escogían en una proporción menor los DIU.

La adolescencia es uno de los grupos etarios identificados como vulnerables en la Estrategia de Salud Sexual y Reproductiva del SNS por las dificultades que puede experimentar para acceder a los servicios sanitarios (1). La provisión de programas de anticoncepción dirigidos específicamente a esta franja etaria, supondría una oportunidad para garantizar la accesibilidad a prácticas de planificación de la reproducción efectivas.

\section{Beneficios y riesgos/carga de las intervenciones}

Los datos aportados por el estudio CHOICE parecen justificar el uso de anticonceptivos reversibles de acción prolongada (LARC) en usuarias adolescentes. Otros tipos de anticonceptivo que requieren un uso diario o rutinario han mostrado una tasa de fallo mucho mayor en esta franja de edad que en mujeres de más edad (17), y las tasas de fallo entre las adolescentes con anticonceptivos como el DIU son mucho menores que con otros métodos (5,1 fallos por 1000 usuarias del DIU LNG/año frente a 60,8 fallos por 1000 usuarias de la píldora/año) (187).

La eficacia de estos métodos en las adolescentes debe valorarse en relación al riesgo de expulsión, estimado alrededor del 10 por cada 100 usuarias/año y es más frecuente que en mujeres de otras edades, e independiente del tipo de dispositivo (202).

Otro aspecto determinante en la elección del DIU como método anticonceptivo en las adolescentes es el del riesgo de ITS. Aunque la tasa de diagnóstico de ITS como la Chlamydia trachomatis es inferior en nuestro entorno que en otros países, es necesario que durante la exploración previa al inicio de un método anticonceptivo se realice una anamnesis completa sobre los hábitos sexuales de las usuarias para determinar el riesgo de una ITS. Los criterios de elegibilidad establecen directrices claras sobre cómo actuar ante esta situación. En situaciones en las que se identifica un mayor riesgo de ITS se ha propuesto el doble método (consistente en la utilización conjunta de un método eficaz para prevenir el embarazo con el preservativo, que previene de las ITS). 


\section{Balance beneficio/riesgo}

Ver apartado anterior.

\section{Uso de recursos y costes}

No se han identificado evaluaciones económicas sobre el uso de DIU o métodos LARC en usuarias adolescentes. Sin embargo en un artículo de revisión narrativa de la literatura sobre el coste de los anticonceptivos LARC identifica la cobertura sanitaria, el pago directo de los anticonceptivos por parte de las usuarias, o la participación de los padres en el pago como las principales barreras para su uso $(207,208)$. Esta publicación destaca las características de los programas comunitarios como el CHOICE, en el que se ofrecían los anticonceptivos sin coste, como una manera de incrementar el uso de los LARC entre las adolescentes, con un impacto directo en la tasa de embarazos no deseados, en resultados de salud, y en el ahorro para el sistema sanitario (207). Aunque no se dispone de una evaluación económica sobre el programa CHOICE, la revisión de Eisenberg 2013 recoge los resultados de un análisis de coste beneficio de un programa de planificación familiar en un estado estadounidense (209). El estudio mostró un considerable beneficio en términos de incremento de uso de los métodos LARC y reducción de los embarazos no deseados y abortos entre adolescentes de entre 14 y 19 años, y calculó un ahorro en los costes derivados de la atención sanitaria de 17,2 dólares estadounidenses por cada dólar invertido en un método anticonceptivo.

En el estudio de coste-efectividad que mostraba la dominancia de los métodos de acción prolongada (métodos más eficaces y menos costosos) para el sistema de salud británico comentado en la pregunta clínica sobre la eficacia de los anticonceptivos, no se realizó ninguna consideración sobre la edad de las usuarias (43).

\section{Justificación}

Se formula una recomendación que destaca los beneficios de los métodos LARC teniendo en cuenta la eficacia que han mostrado estos métodos. La fuerza de la recomendación es débil teniendo en cuenta la calidad de la evidencia disponible, y la necesidad de realizar un balance individualizado entre la eficacia del DIU y otros aspectos como el riesgo de expulsión o ITS. Para esta última situación se ha formulado una recomendación de buena práctica.

\section{Recomendaciones}

\begin{tabular}{|l|l|}
\hline Débil & $\begin{array}{l}\text { Se sugiere ofrecer información a las mujeres adolescentes sobre los dife- } \\
\text { rentes tipos de anticonceptivos, destacando los beneficios adicionales de los } \\
\text { anticonceptivos reversibles de acción prolongada. }\end{array}$ \\
\hline $\begin{array}{l}\text { Buena } \\
\text { práctica } \\
\text { clínica }\end{array}$ & $\begin{array}{l}\text { Debe aconsejarse a las usuarias de DIU realizar una visita de seguimiento } \\
\text { en los primeros meses de uso para valorar su tolerancia, y recordarles que } \\
\text { deben consultar cualquier duda sobre el método anticonceptivo. }\end{array}$ \\
\hline
\end{tabular}




\subsection{Edad reproductiva avanzada}

\subsubsection{Edad de interrupción de la anticoncepción hormonal}

No está establecido hasta qué momento de sus vidas las mujeres mantienen la posibilidad de quedarse embarazadas. A pesar de que la fecundidad disminuye gradualmente a partir de los 32 años, disminución que se acelera tras los 37 años (210), múltiples estudios muestran que mujeres de más de 45 ó 50 años pueden desarrollar un embarazo con buen resultado (211). Por otro lado, la edad de inicio de la menopausia espontánea se sitúa entre los 45 y 55 años, con una media en mujeres españolas situada en torno a los 51 años $(212,213)$.

Aunque la encuesta de fecundidad del Instituto Nacional de Estadística muestra datos de 1999, y no muestra resultados desagregados para los casos de concepción natural, se registraron 5105 embarazos en la franja de edad entre los 40 y los 44 años, y 706 en la edad comprendida entre los 45 a 49 años (214). Por otro lado, en España se registraron durante el año 201387 nacimientos en mujeres de 50 años de edad o más (215). Teniendo en cuenta que no existen medios fiables para confirmar la pérdida definitiva de fertilidad en una mujer, la edad reproductiva tardía de una mujer no puede considerarse un criterio aislado para determinar en qué momento interrumpir el uso de un método anticonceptivo (3). Por lo tanto, la elección de un método anticonceptivo para una mujer debería centrarse en su estado de salud, sus hábitos de vida y su experiencia previa con otros métodos.

La edad materna avanzada se ha asociado consistentemente con complicaciones fetales y obstétricas, que pueden verse agravado por el impacto de los factores asociados a la mayor prevalencia de comorbilidades en las mujeres de esta edad (216). Este hecho debe tenerse en cuenta en el momento de informar a las mujeres en esta etapa de sus vidas sobre el uso de un método anticonceptivo, discutiendo sobre el balance entre los beneficios y riesgos de tener un embarazo no deseado en una edad reproductiva tardía, los beneficios no anticonceptivos asociados a algún método, pero también sobre los eventuales riesgos de la anticoncepción.

Los criterios de elegibilidad permiten valorar todos estos aspectos antes de considerar el uso de un método anticonceptivo (4). Los métodos hormonales combinados, de sólo gestágeno, o los dispositivos intrauterinos son eficaces y seguros (categoría 1 ó 2) cuando se usa la edad ( $\geq 40$ años) como el criterio de elegibilidad del anticonceptivo. No obstante deben considerarse todos aquellos problemas de salud y factores de riesgo que pueden convertir a estos métodos en poco seguros. Por ejemplo, es imprescindible considerar el riesgo de enfermedad cardiovascular (hábito tabáquico, diabetes, hipertensión, antecedentes personales), o la valoración del riesgo o antecedentes de cáncer de mama (217) en la prescripción de determinados métodos combinados.

\subsubsection{Manejo de los dispositivos intrauterinos después de los cuarenta años}

No se han identificado estudios controlados que evalúen el impacto de diferentes modos de manejo en las usuarias de los DIU tras los 40 años de edad. Por este motivo, para el 
desarrollo de esta pregunta clínica se recogen las directrices de la Clinical Effectiveness Unit del Faculty of Sexual and Reproductive Healthcare $(218,219)$ y de la Conferencia de Consenso de la Sociedad Española de Contracepción (8), que realizan una serie de consideraciones sobre el momento de interrupción de los dispositivos intrauterinos.

En estos documentos se recogen una serie de directrices sobre las consideraciones que se deben tener en cuenta cuando se plantea la interrupción de la anticoncepción durante la menopausia. En términos generales se considera que la anticoncepción puede interrumpirse a los 55 años, aunque este criterio debería individualizarse dependiendo de las usuarias, principalmente en aquellas mujeres que tras la edad mencionada mantengan un patrón de sangrado.

En el caso concreto de los métodos no hormonales se considera que puede interrumpirse la anticoncepción tras un año después del último ciclo menstrual en las mujeres mayores de 50 años, periodo que aumenta a los dos años en las mujeres menores de 50 años. El documento de la FSRH considera que las mujeres a las que se ha insertado un DIU de cobre $\geq 300 \mathrm{~mm}^{2}$ a partir de los 40 años, puede mantenerse el dispositivo hasta la menopausia, fuera de la indicación reflejada en la ficha técnica $(218,219)$.

Los resultados derivados de un uso más allá de la indicación del DIU de cobre se evaluaron en un estudio observacional prospectivo, que evaluó Calidad una cohorte de 228 usuarias de un DIU de cobre (TCu 380A) con una edad baja de al menos 35 años (38,8 años de media) en el momento en el que hacía más de 10 años que usaban el dispositivo (220). En las 366 usuarias/año que se evaluaron más allá de los 10 años de uso del dispositivo no se registró ningún embarazo. La principal razón por la cual las usuarias solicitaron interrumpir el método fue porque tuvieron conocimiento que el dispositivo no estaba aprobado para ser utilizado más allá de los 10 años. Tras 10 años de uso, la interrupción del uso del dispositivo a seis años fue de 42,5 por 100 usuarias/año.

En relación a los dispositivos liberadores de levonorgestrel, el documento de la FSRH considera que en las mujeres que han iniciado el uso del DIU a partir de los 45 años pueden continuar con el método durante siete años si presentan un patrón de sangrado aceptable, y además recoge una recomendación de una guía NICE según la cual las usuarias del DIU de levonorgestrel que lo usan a partir de los 45 años y con amenorrea deben continuar con el método hasta la menopausia (221). El documento de la FSRH insiste en la necesidad de informar a estas usuarias sobre la posibilidad de un embarazo espontáneo hasta una edad avanzada, la eficacia del DIU de levonorgestrel y también los riesgos relacionados con la extracción y su reposición. En casos de sangrado o manchado irregular se debe sospechar de actividad folicular y cambiar el dispositivo por un método alternativo (219).

Por su parte, en la Conferencia de Consenso de la Sociedad Española de Contracepción (8), se especifica en las mujeres en las que el DIU de levonorgestrel se insertó entre los 35 y 39 años, éste debería renovarse siguiendo las indicaciones para los que está autorizado. El DIU puede renovarse o sustituirlo por un método alternativo si se sospecha la presencia de ciclos ovulatorios. En las usuarias en las que el DIU de levonorgestrel se in- 
serta tras los 40 años de edad, se conserva las indicaciones de ficha técnica y de la FSRH (actualización 2017) de sustituirlo cada 5 años.

\section{De la evidencia a la recomendación}

\section{Resumen de la evidencia}

No está establecido hasta qué momento en la vida de una mujer mantiene la posibilidad de quedarse embarazada, por lo que la edad no puede ser un motivo aislado para decidir hasta cuándo continuar con un método anticonceptivo. Los criterios de elegibilidad para el uso de anticonceptivos clasifican a los diferentes métodos anticonceptivos hormonales como eficaces y seguros cuando se utiliza la edad como el criterio de elección (4), pero es necesario considerar todos aquellos problemas de salud y factores de riesgo que pueden convertir a estos métodos en poco seguros.

\section{Calidad de la evidencia}

La calidad global es baja.

Los datos valorados para desarrollar la pregunta clínica sobre la edad de interrupción de la anticoncepción hormonal no se han sometido a una evaluación formal de la calidad de la evidencia, debido a que se basan en datos epidemiológicos, poblacionales y clínicos relacionados con la fertilidad de las mujeres, considerando también los criterios de elegibilidad establecidos para la elección de un método anticonceptivo (4).

Los datos sobre el manejo de los dispositivos intrauterinos provienen de las directrices establecidas por otras Sociedades Científicas y se apoyan en la limitada literatura disponible para responder a la pregunta clínica.

\section{Valores y preferencias de las usuarias}

A pesar de no haber identificado estudios específicos aplicables a este apartado, las recomendaciones consideran el beneficio que puede aportar el uso de un método anticonceptivo de acción prolongada en una etapa en la que a pesar de que la fertilidad disminuye, el método mantiene su eficacia en las mujeres que desean evitar un embarazo no deseado. En la valoración de mantener o remplazar los dispositivos intrauterinos se realiza un balance entre los inconvenientes para la usuaria que puede suponer el remplazo de un dispositivo y la eventual pérdida de eficacia del dispositivo cuando se utiliza más allá de la indicación para el que está aprobado. Del mismo modo, especialmente en el caso del dispositivo liberador de levonorgestrel, se individualiza la decisión teniendo en cuenta el patrón de sangrado de las usuarias.

\section{Beneficios y riesgos/carga de las intervenciones}

Ver apartado anterior. 


\section{Balance beneficio/riesgo}

Ver apartado anterior.

\section{Uso de recursos y costes}

No se han identificado evaluaciones económicas relevantes aplicables a estas preguntas clínicas.

\section{Justificación}

Las recomendaciones relacionadas con la edad hasta la que se puede continuar con un método anticonceptivo se han basado en los datos disponibles sobre fertilidad de las mujeres en edades avanzadas. La recomendación sobre la edad de las usuarias se ha graduado como fuerte teniendo en cuenta que los beneficios de la anticoncepción superan a los riesgos en las mujeres fértiles que usan los métodos disponibles para evitar un embarazo no deseado. Esta recomendación se ha complementado con aspectos recogidos en otros documentos de recomendaciones que abordan el manejo de la anticoncepción en mujeres de edades avanzadas o en los criterios de elegibilidad para el uso de anticonceptivos (FSRH 2010, $3)$. En estos casos, se han clasificado las recomendaciones como de buena práctica clínica.

\section{Recomendaciones}

\begin{tabular}{|l|l|}
\hline Fuerte & $\begin{array}{l}\text { Se recomienda no utilizar la edad de la usuaria como el único criterio en la } \\
\text { decisión sobre la interrupción de un anticonceptivo. }\end{array}$ \\
\hline $\begin{array}{l}\text { Buena } \\
\text { práctica } \\
\text { clínica }\end{array}$ & $\begin{array}{l}\text { Se sugiere que, en mujeres sin factores de riesgo ni problemas médicos rele- } \\
\text { vantes que desean evitar un embarazo no deseado, se mantenga la anticon- } \\
\text { cepción hormonal hasta la menopausia. }\end{array}$ \\
\hline $\begin{array}{l}\text { Buena } \\
\text { práctica } \\
\text { clínica }\end{array}$ & $\begin{array}{l}\text { Se sugiere que en la decisión de utilizar o continuar un determinado método } \\
\text { anticonceptivo en mujeres con edad reproductiva tardía, se individualice la } \\
\text { decisión teniendo en cuenta sus riesgos y beneficios, incluidos los no anti- } \\
\text { conceptivos. }\end{array}$ \\
\hline $\begin{array}{l}\text { Buena } \\
\text { práctica } \\
\text { clínica }\end{array}$ & $\begin{array}{l}\text { Se sugiere que las usuarias de un método anticonceptivo no hormonal como } \\
\text { el DIU de cobre interrumpan su uso transcurridos dos años tras el último } \\
\text { ciclo menstrual en las mujeres menores de 50 años, periodo que puede ser } \\
\text { de un año en las mujeres mayores de 50 años. }\end{array}$ \\
\hline $\begin{array}{l}\text { Buena } \\
\text { práctica } \\
\text { clínica }\end{array}$ & $\begin{array}{l}\text { Se sugiere informar a las usuarias de un DIU de cobre insertado a partir de } \\
\text { los 40 años sobre la posibilidad de mantener el dispositivo hasta la aparición } \\
\text { de la menopausia. }\end{array}$ \\
\hline $\begin{array}{l}\text { Buena } \\
\text { práctica } \\
\text { clínica }\end{array}$ & $\begin{array}{l}\text { Se sugiere extraer el DIU de levonorgestrel entre los 40 y 44 años a aquellas } \\
\text { mujeres a las que se les había insertado entre los 35 y 39 años. El DIU puede } \\
\text { renovarse o sustituirlo por un método alternativo. }\end{array}$ \\
\hline $\begin{array}{l}\text { Buena } \\
\text { práctica } \\
\text { clínica }\end{array}$ & $\begin{array}{l}\text { Se sugiere mantener el DIU de levonorgestrel durante siete años o hasta la } \\
\text { aparición de la menopausia en las usuarias en las que se les insertara el dis- } \\
\text { positivo a partir de los 45 años de edad. }\end{array}$ \\
\hline
\end{tabular}




\subsection{Sobrepeso y obesidad}

\subsubsection{Consideraciones en usuarias de anticonceptivos hormonales o intrauterinos con sobrepeso u obesidad}

Para la elaboración de este apartado se han integrado las indicaciones recogidas en los documentos sobre los criterios de elegibilidad para la anticoncepción de la Organización Mundial de la Salud (4) y los del UK Medical Eligibility Criteria-UKMEC (5), además de los recogidos en la guía de práctica clínica del Royal College of Obstetricians and Gynaecologist británico (222) sobre el riesgo de enfermedad tromboembólica venosa (ETV) asociado a la contracepción hormonal. Otra revisión ha recogido varios estudios observacionales para intentar contrastar las indicaciones que recogen estos criterios de elegibilidad (223).

Adicionalmente se ha identificado una revisión de la literatura narrativa (224) que discute sobre la contracepción en circunstancias especiales, y un documento de consenso basado en una búsqueda de la literatura científica que recoge consideraciones especiales sobre la contracepción en mujeres obesas (225).

Por otro lado una revisión sistemática Cochrane ((226), fecha de búsqueda enero de 2013) evaluó la eficacia de los anticonceptivos hormonales en mujeres con sobrepeso u obesidad frente aquellas con un IMC menor. Se ha obtenido información sobre los dispositivos intrauterinos de un documento de recomendaciones de la Sociedad Europea de Anticoncepción (228) y de un análisis de la cohorte del estudio CHOICE para el implante de etonogestrel (229).

La obesidad se ha relacionado fundamentalmente con un incremento del riesgo de enfermedad tromboembólica venosa (ETV). El riesgo puede llegar a ser 10 veces mayor en las mujeres con obesidad que toman anticonceptivos hormonales combinados en comparación con el de las mujeres que no son obesas y no los usan (222). Aunque en situaciones específicas, el riesgo sobrepasa el beneficio, las mujeres adultas o adolescentes con un índice de masa corporal (IMC) entre los $30 \mathrm{~kg} / \mathrm{m}^{2}$ y los $34 \mathrm{~kg} / \mathrm{m}^{2}$ pueden ser usuarias sin restricción de los contraceptivos hormonales combinados (contraceptivos orales combinados, parche y anillo vaginal) $(4,225)$. Por el contrario, en las mujeres con un IMC que supere los $35 \mathrm{~kg} / \mathrm{m}^{2}$ el uso de anticonceptivos hormonales combinados está contraindicado por un aumento importante del riesgo de $\operatorname{ETV}(4,225)$.

Debido a que el riesgo de ETV también aumenta con la edad y en personas con un hábito tabáquico, es recomendable que las mujeres con obesidad y alguno de estos factores de riesgo opten por anticonceptivos de progestágeno solo o por el DIU (224).

A pesar de estas indicaciones, una revisión (223) revisa cinco estudios de casos y controles en los que se evalúa el efecto de los anticonceptivos hor-

Calidad baja monales combinados sobre el riesgo de ETV en relación al IMC (230-234). Esta revisión de la literatura pretende determinar si el riesgo de ETV entre las usuarias de anticonceptivos hormonales combinados aumenta de manera significativa entre los IMC de 30 a 34,35 a 39 , ó $>$ de $40 \mathrm{~kg} / \mathrm{m}^{2}$ en un intento por matizar los criterios de eligibilidad de la OMS y UKMEC. 
La siguiente tabla resume el riesgo relativo de ETV asociado a los anticonceptivos hormonales combinados por cada intervalo de IMC descrito en los estudios recopilados por Trussell 2008 (223). Estas estimaciones del efecto se derivan de la comparación con el riesgo de ETV en mujeres con un IMC entre los 25 y los $29 \mathrm{~kg} / \mathrm{m}^{2}$.

\begin{tabular}{|c|c|c|c|c|}
\hline $\begin{array}{l}\mathrm{IMC} \\
\mathrm{kg} / \mathrm{m}^{2}\end{array}$ & $\begin{array}{c}\text { Abdollahi } 2003 \\
\text { (230) } \\
\text { Dinamarca }\end{array}$ & $\begin{array}{c}\text { Pomp } 2007 \text { (233) } \\
\text { Holanda }\end{array}$ & $\begin{array}{l}\text { Nightingale } \\
2000 \text { (232) } \\
\text { Reino Unido }\end{array}$ & $\begin{array}{c}\text { Lidegaard } \\
2002(231) \\
\text { Holanda }\end{array}$ \\
\hline$<20$ (delgadez) & \multirow{2}{*}{0,5} & \multirow{2}{*}{0,4} & 0,6 & 0,5 \\
\hline $20-24$ (normal) & & & 0,7 & 0,6 \\
\hline $\begin{array}{l}25-29 \\
\text { (sobrepeso) }\end{array}$ & \multicolumn{4}{|c|}{ categoría de referencia $=1$} \\
\hline $\begin{array}{l}30-34 \\
\text { (obesidad) }\end{array}$ & \multirow{2}{*}{1,0} & \multirow{2}{*}{2,0} & 1,3 & \multirow{2}{*}{2,7} \\
\hline $\begin{array}{l}\geq 35 \text { (obesidad } \\
\text { mórbida) }\end{array}$ & & & 2,2 & \\
\hline Casos & 170 & 676 & 384 & 987 \\
\hline Controles & 170 & 538 & 1464 & 4054 \\
\hline
\end{tabular}

Tal como se observa en la tabla, los resultados fueron contradictorios entre los estudios. Mientras que en el estudio de Abdollahi 2003 no se observó un incremento del riesgo (230), los dos estudios holandeses mostraron un incremento del riesgo de ETV considerable $(231,233)$. En el estudio británico se observó un riesgo considerable solamente en las mujeres con un $\mathrm{IMC} \geq 35$.

En la siguiente tabla se incluye el riesgo relativo de ETV de las usuarias de anticonceptivos hormonales combinados comparado con el de las no usuarias, según el IMC.

\begin{tabular}{|l|c|c|c|}
\hline \multicolumn{1}{|c|}{$\begin{array}{c}\text { IMC } \\
\mathbf{k g} / \mathbf{m}^{\mathbf{2}}\end{array}$} & $\begin{array}{c}\text { Abdollahi 2003 } \\
\mathbf{( 2 3 0 )} \\
\text { Dinamarca }\end{array}$ & $\begin{array}{c}\text { Nightingale 2000 } \\
\mathbf{( 2 3 2 )} \\
\text { Reino Unido }\end{array}$ & $\begin{array}{c}\text { Pomp 2007 } \\
\mathbf{( 2 3 3 )} \\
\text { Holanda }\end{array}$ \\
\hline$<25$ & 4,6 & \multirow{2}{*}{$3,3(2,0-5,5)$} & $4,3(2,9-6,2)$ \\
\cline { 1 - 2 } $25-29$ (sobrepeso) & 11,3 & $4,8(2,6-9,1)$ \\
\hline$\geq 30$ (obesidad) & 6,5 & $6,0(3,1-11,7)$ & $8,4(4,0-17,4)$ \\
\hline Casos & 170 & 196 & 676 \\
\hline Controles & 170 & 746 & 538 \\
\hline
\end{tabular}


En la revisión de Trussell 2008 se argumenta que a pesar del aumento de riesgo de ETV entre las mujeres con un mayor IMC, los intervalos de confianza entre las diferentes categorías de IMC en los estudios de Nightingale 2000 y Pomp 2007 se solapan y las diferencias entre las estimaciones no alcanzan la significación estadística $(223,232,233)$. Además destaca la necesidad de disponer de valores absolutos para valorar adecuadamente la relevancia de las diferencias en el riesgo de ETV entre los diferentes intervalos de IMC. Para disponer de estos datos se extrapola la incidencia descrita en el estudio de Nightingale 2000 (39 casos de ETV por 100.000 mujeres/año) para estimar el riesgo absoluto anual (por 100.000) de ETV en las usuarias de anticonceptivos hormonales combinados a partir de los datos de este estudio (232):

\begin{tabular}{|c|c|c|c|c|c|}
\hline $\begin{array}{l}\mathrm{IMC} \\
\mathrm{kg} / \mathrm{m}^{2}\end{array}$ & $\begin{array}{c}\text { Número de } \\
\text { participantes }\end{array}$ & OR & $\begin{array}{c}\text { Riesgo } \\
\text { absoluto }\end{array}$ & $\begin{array}{l}\text { Criterios de } \\
\text { elegibilidad } \\
\text { UKMEC (5) }\end{array}$ & $\begin{array}{c}\text { Criterios de } \\
\text { elegibilidad } \\
\text { OMS (4) }\end{array}$ \\
\hline$<20$ (delgadez) & 194 & 0,8 & 27,0 & \multirow{3}{*}{1} & \multirow{3}{*}{1} \\
\hline $20-24$ (normal) & 681 & 1,0 & 33,7 & & \\
\hline $\begin{array}{l}25-29 \\
\text { (sobrepeso) }\end{array}$ & 216 & 1,4 & 47,2 & & \\
\hline 30 - 34 (obesidad) & 77 & 1,8 & 60,7 & 2 & 2 \\
\hline $\begin{array}{l}\geq 35 \text { (obesidad } \\
\text { mórbida) }\end{array}$ & 27 & 3,1 & 104,5 & 3 & 3 \\
\hline
\end{tabular}

Teniendo en cuenta los estudios recopilados por Trussell 2008, el riesgo absoluto de ETV entre las usuarias de anticonceptivos hormonales con un IMC $\geq 35 \mathrm{~kg} / \mathrm{m}^{2}$ oscilaría entre el 63 y el 176 por cada 100.000 mujeres/año (en el caso de ETV fatal los autores estiman un riesgo del 2,4 por cada 100.000 mujeres/año) (223). Los autores del estudio comentan que este riesgo es similar a la de otros factores a tener en cuenta con un riesgo absoluto similar (edad de la usuaria) o superior (tabaquismo) al de la obesidad. Los autores consideran, por tanto, que se aplican criterios inconsistentes en el documento UKMEC y el de la OMS para los diferentes factores comentados.

Por otro lado, la obesidad es un factor de riesgo cardiovascular reconocido. En mujeres en las que, además de la obesidad, se identifiquen otros factores de riesgo como edad avanzada, tabaquismo, diabetes o hipertensión, los métodos anticonceptivos hormonales combinados (píldora, parche, anillo) están contraindicados, ya que el riesgo de enfermedad cardiovascular secundaria se incrementa sustancialmente $(4,225)$.

Una guia clínica focalizada en aspectos relacionados con la contracepción en mujeres con obesidad de la Society of Family Planning estadounidense (225) discute sobre diferentes aspectos como el riesgo de embarazo en estas mujeres, o el mismo riesgo de ETV.

Aunque la obesidad se reconoce como un factor de riesgo para la reducción de la 
fertilidad, la mayoría de mujeres con obesidad ovulan regularmente y pueden quedarse embarazadas y mantienen una actividad sexual similar a la del resto de mujeres (235). Por otro lado, un estudio transversal en un registro estadounidense de cerca de 8000 participantes mostró que las mujeres obesas usaban anticonceptivos con menor frecuencia que el resto de mujeres (236). En el caso de las adolescentes, algunos estudios observacionales han mostrado que, debido a una insatisfación con la imagen corporal, las adolescentes obesas pueden tener un mayor riesgo a mantener prácticas sexuales de riesgo. Por estas razones, la guía de la Society of Family Planning concluye que las mujeres obseas tienen un mayor riesgo de quedarse embarazadas que las mujeres con un IMC normal.

Respecto al riesgo de ETV, la guía de la Society of Family Planning se desmarca en cierto sentido de las recomendaciones de los documentos de eligibilidad de la anticoncepción FSRH - UKMEC (5) y OMS (4). Tanto el uso de anticonceptivos hormonales (237) como la obesidad (230) aumentan el riesgo de ETV, pero la guía comenta que en mujeres con obesidad sanas, el uso de anticonceptivos hormonales combinados (píldora, parche o anillo) aumenta de manera moderada el riesgo de ETV en relación a las usuarias de anticonceptivos hormonales no obesas, pero esto no debe ser un motivo para contraindicarlos puesto que el riesgo es el mismo que el asociado al embarazo (225), y mucho mayor inmediatamente tras el parto (245).

\subsubsection{Eficacia de los anticonceptivos hormonales en usuarias con sobrepeso u obesidad}

La revisión Cochrane sobre la eficacia de los anticonceptivos hormonales en mujeres con sobrepeso u obesidad (226), incluyó resultados de 13 ensayos clínicos (con un total de 49.712 mujeres participantes). Entre cinco estudios que compararon la eficacia de los anticonceptivos entre mujeres con sobrepeso u obesidad frente a mujeres con un IMC menor, solamente un estudio mostró un aumento de los embarazos entre las mujeres con sobrepeso. Las mujeres en este estudio usaban un anticonceptivo combinado con $1,0 \mathrm{mg}$ de acetato de noretindrona y $20 \mu \mathrm{g}$ de EE (227) y mostraba una mayor tasa de embarazo entre aquellas mujeres con un IMC de $25 \mathrm{~kg} / \mathrm{m}^{2}$ o superior en comparación con las mujeres con un IMC inferior (RR 2,49; IC95\% $1,01$ a 6,13$)$. El resto de estudios evaluaron otros anticonceptivos hormonales combinados, el parche transdérmico, un anticonceptivo implantable $\mathrm{y}$ un inyectable.

En el ensayo clínico que evaluó la eficacia del parche transdérmico se registraron 15 embarazos en las 3319 participantes en el estudio (238). Siete de los 15 embarazos se registraron en el decil más alto de peso, en mujeres con $80 \mathrm{~kg}$ o más. Cinco de estos siete embarazos se registraron en mujeres con $90 \mathrm{~kg}$ de peso o más ( $3 \%$ del total de las participantes en el estudio). En este estudio, el peso al inicio del estudio se asoció significativamente con el riesgo de embarazo en un modelo de riesgos proporcionales de Cox $(\mathrm{P}<0,001)$ en el que se evaluaron otros factores como edad, o IMC.

Calidad baja 
Dos ensayos clínicos evaluaron la eficacia del inyectable de medroxiprogesterona, sin que se registrara ningún embarazo al año de uso (239). En estos estudios solamente el $27 \%$ de usuarias tenían sobrepeso u obesidad en el grupo de participantes en centros europeos o asiáticos, mientras que en los centros estadounidenses el porcentaje ascendía hasta el $40 \%$.

La literatura científica no muestra en términos generales una asociación del IMC con la eficacia de los anticonceptivos hormonales, y se dispone de datos limitados para cada método anticonceptivo. Si bien los estudios que evalúan IMC (en lugar del peso) pueden dar más información acerca de si la composición corporal se relaciona con la eficacia anticonceptiva, no se dispone de suficiente información al respecto.

El uso del DIU de cobre en mujeres con sobrepeso u obesidad no debería plantear problemas debido a que el método no supone ningún aspecto metabólico, no plantea problemas relacionados con el riesgo de ETV y su acción anticonceptiva actúa principalmente en el útero. Para su uso deben considerarse tanto sus contraindicaciones (embarazo, malformaciones uterinas, o enfermedad pélvica inflamatoria) como situaciones en las que deberían valorarse sus beneficios y riesgos (riesgo de ITS, histerometría $>9 \mathrm{~cm}$, nuliparidad) (228).

El DIU de levonorgestrel aportaría las mismas ventajas, puesto a que tiene una acción local en la cavidad uterina y no se asocia con un aumento del riesgo de ETV. Por otro lado un estudio observacional prospectivo en 56 mujeres con un IMC $\geq 30 \mathrm{~kg} / \mathrm{m}^{2}$ a las que se insertó un DIU de levonorgestrel debido a un sangrado uterino anormal, mostraron una mejora del sangrado entre los tres y seis meses de uso, con un porcentaje de expulsión de algo más del 20\% (240).

Un análisis de la cohorte del estudio CHOICE Project evaluó la eficacia del implante de etonogestrel en mujeres con sobrepeso u obesidad (229). De las usuarias de este implante en la cohorte, el 28\% tuvieron sobrepeso y el $35 \%$ obesidad. Solamente se registró un embarazo entre las 1377 usuarias por año de uso del implante, en una mujer con IMC de $30,7 \mathrm{~kg} / \mathrm{m}^{2}$. La tasas de fallo del implante para las mujeres con sobrepeso fue de 0 por cada 100 mujeres/año, y de 0,23 por cada 100 mujeres/año en las mujeres con obesidad. Un análisis de supervivencia no mostró diferencias en la eficacia del método dependiendo del IMC de las usuarias.

Por otro lado, el implante de desogestrel es un método seguro puesto que no aumenta de manera significativa el riesgo de ETV, sin que se disponga de datos sobre una alteración de su eficacia en mujeres con sobrepeso u obesidad (228). 


\section{De la evidencia a la recomendación}

\section{Resumen de la evidencia}

El uso de anticonceptivos hormonales combinados en las mujeres con un índice de masa corporal de $35 \mathrm{~kg} / \mathrm{m}^{2}$ o superior está contraindicado debido a un riesgo considerable de enfermedad tromboembólica venosa $(4,225)$, aunque algunos autores han considerado que se aplican unos criterios de estimación de riesgo más estrictos en el caso de la obesidad que en el de otros factores como la edad de las usuarias o el tabaquismo (223).

La literatura científica no muestra en términos generales una asociación del IMC con la eficacia de los anticonceptivos hormonales, y se dispone de datos limitados para cada método anticonceptivo $(226,228)$. Los datos

Calidad baja

Calidad baja más relevantes muestran una mayor tasa de embarazo en las usuarias de un anticonceptivo hormonal combinado de noretindrona en mujeres con un IMC superior a $25 \mathrm{~kg} / \mathrm{m}^{2}$ (227), y un aumento del riesgo de embarazo en las usuarias del parche transdérmico con un peso superior a los $90 \mathrm{~kg}$ (238).

\section{Calidad de la evidencia}

La calidad global es baja.

En el caso de la relación de la obesidad con el riesgo de enfermedad tromboembólica venosa, las pruebas se han obtenido de estudios de casos y controles con resultados heterogéneos, sin motivos para considerar aumentar la calidad de la evidencia.

En el caso de la eficacia de los anticonceptivos hormonales combinados, la calidad de la evidencia es baja debido a serias limitaciones en el diseño del estudio (el estudio de Burkman 2009 no realizó ajustes a factores de confusión adecuados, y los análisis de los resultados dependiendo del peso y el IMC de las participantes fueron post hoc (227)). En el caso de la eficacia del parche y el inyectable, los estudios también tuvieron limitaciones en el diseño. Teniendo en cuenta que además la información es limitada para los diferentes tipos de anticonceptivos, la calidad de la evidencia se ha clasificado como baja.

\section{Valores y preferencias de las usuarias}

En un estudio transversal que analizó datos de la National Survey of Family Growth estadounidense, se analizaron los factores asociados con el uso incorrecto de los métodos anticonceptivos por parte de las usuarias con obesidad (mujeres sexualmente activas de entre 20 y 44 años con un IMC de $30 \mathrm{~kg} / \mathrm{m}^{2}$ o superior) (241). Entre otros resultados, el estudio mostró que solamente el $42 \%$ de las participantes en el estudio (el $20 \%$ en el caso de las usuarias de un anticonceptivo que no requiere prescripción) habían discutido con un profesional sanitario sobre la eficacia de los diferentes anticonceptivos. Las usuarias de anticonceptivos que no requieren prescripción tenían una probabilidad menor de haber discutido con un profesional sanitario sobre la eficacia de los diferentes métodos en com- 
paración con las usuarias de la píldora, parche, anillo, inyectable, implante o DIU (RR en no usurias: 0,16; IC95\% 0,10 a 0,27; RR en usuarias de un método de barrera: 0,15; IC95\% $0,09$ a 0,25$)$.

\section{Beneficios y riesgos/carga de las intervenciones}

No se ha considerado este aspecto en esta pregunta clínica.

\section{Balance beneficio/riesgo}

En la elección de un método anticonceptivo para mujeres con sobrepeso u obesidad debe tenerse en cuenta que éstas tienen un riesgo más alto de enfermedad tromboembólica venosa, considerando este aspecto con la eficacia de los anticonceptivos que no varía de manera significativa en comparación con las mujeres con IMC menores.

\section{Uso de recursos y costes}

No se ha considerado este aspecto en esta pregunta clínica.

\section{Justificación}

El principal aspecto a tener en cuenta en la formulación de una recomendación en este apartado es el balance entre el mayor riesgo de enfermedad tromboembólica venosa en las mujeres con sobrepeso u obesidad (que lleva a la contraindicación en la elegibilidad de algunos métodos) y la eficacia de los métodos anticonceptivos, que no sufre modificaciones notables en comparación con el resto de usuarias. En términos generales, cuando en las rescomendaciones se hace referencia a mujeres con obesidad se toma como referencia el valor reflejado en los criterios de elegibilidad de la OMS (IMC $\geq 30 \mathrm{Kg} / \mathrm{m}^{2}$ ).

\section{Recomendaciones}

\begin{tabular}{|l|l|}
\hline $\begin{array}{l}\text { Buena } \\
\text { práctica } \\
\text { clínica }\end{array}$ & $\begin{array}{l}\text { En la elección de un método anticonceptivo para una mujer con sobrepeso } \\
\text { u obesidad se sugiere individualizar la decisión de acuerdo a las caracterís- } \\
\text { ticas de cada usuaria y a las directrices reflejadas en los criterios médicos de } \\
\text { elegibilidad para el uso de anticonceptivos, informando a la usuaria de las } \\
\text { ventajas e inconvenientes de cada uno de los métodos. }\end{array}$ \\
\hline Fuerte & $\begin{array}{l}\text { No se recomienda el uso de anticonceptivos hormonales combinados a las } \\
\text { mujeres con obesidad en las que se identifican factores de riesgo cardiovas- } \\
\text { cular adicional (edad avanzada, tabaquismo, diabetes, hipertensión), debido } \\
\text { a que aumenta el riesgo de sufrir alguna enfermedad cardiovascular secun- } \\
\text { daria. }\end{array}$ \\
\hline Débil & $\begin{array}{l}\text { En estas mujeres pueden considerarse métodos anticonceptivos que han } \\
\text { mostrado una buena eficacia sin aumentar el riesgo cardiovascular, como } \\
\text { los dispositivos intrauterinos o los métodos hormonales de sólo gestágeno. }\end{array}$ \\
\hline
\end{tabular}




\subsection{Puerperio}

\subsubsection{Inicio de la anticoncepción hormonal en el puerperio}

Este apartado recoge las recomendaciones de la "Guía de Práctica Clínica de Atención en el Embarazo y Puerperio" del programa de Guías de Práctica Clínica en el SNS (Guía de Embarazo y Puerperio, en adelante) (242). La Guía basó sus recomendaciones en una revisión sistemática sobre la recuperación de la fertilidad tras el parto (243), los criterios de elegibilidad para el uso de anticonceptivos $(3,4)$ y otros documentos de directrices disponibles (244). En el desarrollo de esta pregunta clínica, además se han valorado los datos de otras revisiones sistemáticas sobre el riesgo de tromboembolismo durante el puerperio (245) o el impacto de los anticonceptivos de sólo gestágeno en la lactancia y en los resultados neonatales (246).

Una revisión sistemática incluyó cuatro estudios observacionales en los que Calidad se evaluaron el periodo de tiempo entre el parto y la primera menstruación baja y la ovulación en mujeres no lactantes (243). En los estudios la primera menstruación tuvo lugar en un rango entre los 45 y los 64 días y la primera ovulación entre los 45 y 94 días. Hasta el $71 \%$ de las menstruaciones fueron precedidas de ovulación y hasta el $60 \%$ fueron potencialmente fértiles.

A pesar de que el tiempo medio hasta la ovulación fue de 45 días de media tras el parto (con una desviación estándar de 16,9 días), dos estudios recogieron ovulaciones más tempranas que fueron juzgadas por los investigadores como fértiles, subrayando la necesidad de valorar el inicio apropiado de la anticoncepción tras el parto en las mujeres que deseen evitar un embarazo no deseado.

Los criterios de elegibilidad para el uso de anticonceptivos y otros documentos de directrices consideran que los anticonceptivos no son necesarios durante los primeros 21 días tras el parto. Si se inicia un tratamiento anticonceptivo hormonal después de los 21 días tras el parto, se debe descartar embarazo, y aconsejar la abstinencia sexual o el uso de un método anticonceptivo adicional durante los primeros siete días de uso, dos en el caso de anticonceptivos con progestágenos solos.

Aquellas mujeres con amenorrea y que realizan lactancia materna exclusiva, pueden evitar el uso de métodos contraceptivos hasta los 6 meses tras el parto, siguiendo el denominado método lactancia-amenorrea (MELA) $(3,4,244)$. Es importante tener en cuenta que en las mujeres que optan por el MELA el riesgo de embarazo aumenta cuando la frecuencia de la lactancia disminuye (al interrumpir algunas tomas, o al iniciar la lactancia mixta y la introducción de alimentos), momento en el que las mujeres pueden volver a menstruar.

La Guía de Embarazo y Puerperio también recogió los resultados de una revisión Cochrane sobre la eficacia del MELA ((247), fecha de búsqueda Calidad 2008). La revisión incluyó 12 estudios observacionales prospectivos, $10 \sin$ baja grupo control y 2 con grupo control. 
En dos estudios observacionales controlados se describió una tasa de embarazos a los 6 meses de seguimiento del $0,45 \%$ y $2,45 \%$, mientras que los estudios no controlados describieron un rango del 0 al 7,5\%.

En los estudios que se restringieron a las mujeres que realizaban lactancia materna exclusiva con amenorrea y que no usaban ningún método anticonceptivo la tasa de embarazo a los seis meses varió entre el 0,88 y el 1,2 (el IC95\% en uno de los estudios fue de 0 a $2,4 \%$ ).

La Guía de Embarazo y Puerperio recoge las indicaciones de los diferentes métodos anticonceptivos de la propuesta de los criterios de elegibilidad de la OMS (242), que se han contrastado en esta pregunta clínica con los del Centers for Disease Control and Prevention (3). Teniendo en cuenta que parámetros de coagulación y fibrinólisis se normalizan aproximadamente a las 3 semanas tras el parto, se puede iniciar un tratamiento con anticonceptivos hormonales combinados tras tres semanas si las mujeres no realizan lactancia materna y si no existen contraindicaciones para este método. En las mujeres con riesgo de tromboembolismo venoso, el periodo debería extenderse a los 42 días, y en las mujeres que realizan lactancia materna se desaconseja su uso hasta pasados los seis meses mínimos recomendados para la lactancia.

Una revisión sistemática recogió los datos de incidencia y el riesgo de tromboembolismo venoso durante el postparto de 13 estudios observacionales Calidad (245). Dos de los estudios compararon el riesgo de tromboembolismo de las mujeres en las seis primeras semanas tras el parto con las de mujeres no embarazadas, y en ambos se observó un incremento muy significativo del riesgo durante el puerperio (uno de los estudios mostró un RR de 21,5 sin que los autores pudieran calcular el IC95\%, mientras que el otro mostró un OR 84; IC95\% 31,7 a 222,6).

Las tasas de incidencia de tromboembolismo recogidas en tres estudios oscilaron entre el 25 y el 99 por 10000 mujeres/año, mayores que las recogidas en la literatura en mujeres no embarazadas (entre 2,5 y 21,5). La incidencia de tromboembolismo fue mayor inmediatamente tras el parto (tasa de incidencia estandarizada de 115,1; IC95\% 96,4 a 137,0), aunque se moderaba tras las cuatro y seis semanas tras el parto.

Los anticonceptivos de sólo gestágeno pueden usarse en cualquier momento tras el parto. Las mujeres que realizan lactancia materna pueden utilizar este método sin restricción tras las seis semanas postparto. Antes de las seis semanas los beneficios todavía son mayores que los riesgos (categoría 2 en los criterios de elegibilidad) (4).

Una revisión sistemática evaluó el impacto de los anticonceptivos de sólo gestágeno en la lactancia y en los resultados neonatales ((246), fecha de búsqueda mayo de 2009), incluyendo cinco ensayos clínicos y 38 estudios Calidad baja observacionales. 
En términos generales los estudios no mostraron que los diferentes métodos de sólo gestágeno tengan un impacto sobre el desarrollo de la lactancia durante los 12 primeros meses de vida del bebé. Los estudios tampoco mostraron un impacto de los anticonceptivos en el crecimiento de los bebés, su salud o desarrollo con datos que oscilaron entre los seis meses y los seis años de seguimiento.

Los criterios de elegibilidad de la OMS también destacan que los estudios disponibles no han mostrado un impacto de los anticonceptivos de sólo gestágeno en el desarrollo de la lactancia materna o el desarrollo de los bebés, aunque insisten en que los estudios sufren de importantes limitaciones y pueden no ser válidos para conocer los riesgos de la exposición a largo plazo (4).

Los criterios de elegibilidad para el uso de anticonceptivos de la OMS no sugieren ninguna restricción a la inserción de un DIU cuando se realiza más allá de las 4 semanas tras el parto, o si se realiza en las primeras 48 horas tras el parto en mujeres que no tienen planeado realizar lactancia materna $(3,4)$. La inserción temprana del DIU de levonorgestrel debería evitarse en las mujeres que deseen instaurar la lactancia materna. Entre las 48 horas y cuatro semanas después del parto se desaconseja la inserción de dispositivos intrauterinos (4), y se contraindican en caso de presencia de sepsis puerperal $(3,4)$. La usuarias de un DIU deben ser informadas de los signos de expulsión, debiendo acudir a la consulta para su revisión o si se detectan problemas.

El estudio de cohortes EURAS-IUD (European Active Surveillance Study for Intrauterine Devices) ha valorado la incidencia de las perforaciones uteCalidad rinas derivadas del uso rutinario de los DIU. El estudio se llevó a cabo en baja seis países europeos e incluyó a 61.500 usuarias del DIU de levonorgestrel o el DIU de cobre (248).

El estudio mostró una incidencia de perforaciones del 1,3 por cada 1.000 dispositivos insertados (IC95\% 1,1 a 1,6), sin que hubiera diferencias dependiendo del tipo de dispositivo (1,4 por cada 1.000 inserciones de un DIU de levonorgestrel (IC95\% 1,1 a 1,8) frente a 1,1 por cada 1.000 inserciones de un DIU de cobre (IC95\% 0,7 a 1,6).

Independientemente del tipo de dispositivo insertado, el estudio identificó un incremento del riesgo de perforación en las mujeres lactantes en el momento de la inserción (en comparación a las no lactantes, y en las mujeres en las que la inserción de producía durante las 36 semanas después de dar a luz (en comparación a la inserción más tardía). La siguiente tabla muestra la incidencia de perforación por cada 1.000 inserciones de dispositivos de acuerdo a las características comentadas (248): 


\begin{tabular}{|l|c|c|c|}
\hline & $\begin{array}{c}\text { Mujer lactante en } \\
\text { el momento de la } \\
\text { inserción }\end{array}$ & $\begin{array}{c}\text { Mujer no lactante } \\
\text { en el momento de la } \\
\text { inserción }\end{array}$ & $\begin{array}{c}\text { Riesgo de } \\
\text { perforación } \\
\text { RR (IC95\%) }\end{array}$ \\
\hline $\begin{array}{l}\text { Inserción antes de las } \\
\mathbf{3 6} \text { semanas tras el } \\
\text { parto }\end{array}$ & $\begin{array}{c}6,5 \text { perforaciones } \\
(\text { IC95\% 3,9 a } 7,9)\end{array}$ & $\begin{array}{c}1,7 \text { perforaciones } \\
(\text { IC95\% 0,8 a 3,1) }\end{array}$ & $\begin{array}{c}3,3 \\
(1,6 \text { a } 6,7)\end{array}$ \\
\hline $\begin{array}{l}\text { Inserción después de } \\
\text { las 36 semanas tras } \\
\text { el parto }\end{array}$ & $\begin{array}{c}1,6 \text { perforaciones } \\
(\text { IC95\% 0,0 a 9,1) }\end{array}$ & $\begin{array}{c}0,7 \text { perforaciones } \\
(\text { IC95\% } 0,5 \text { a } 1,1)\end{array}$ & $\begin{array}{c}2,2 \\
(0,3 \text { a 16,3) }\end{array}$ \\
\hline $\begin{array}{l}\text { Riesgo de } \\
\text { perforación } \\
\text { RR (IC95\%) }\end{array}$ & 3,4 & $\begin{array}{c}2,3 \\
(1,1 \text { a 4,7) }\end{array}$ & \\
\hline
\end{tabular}

En una carta de seguridad dirigida a los profesionales sanitarios para comunicar este aspecto de seguridad (249), se destaca la baja incidencia de perforación que se deriva de la inserción de un DIU y los factores comentados que pueden aumentar el riesgo. Se anima a los profesionales sanitarios a informar a las usuarias de la eficacia de este método a largo plazo y del incremento del riesgo de perforación ante estas circunstancias antes de la inserción de un DIU, además de los síntomas de alerta de perforación.

\section{De la evidencia a la recomendación}

\section{Resumen de la evidencia}

La mayoría de las mujeres presentan la primera ovulación y la primera menstruación más allá de los primeros 45 días tras el parto. No obstante en la literatura se han identificado casos de ovulaciones, consideradas fértiles, mucho más tempranas (243).

Los criterios de elegibilidad para el uso de anticonceptivos y otros documentos de directrices consideran que los anticonceptivos no son necesarios durante los primeros 21 días tras el parto.

La tasa de embarazo en mujeres con amenorrea y lactancia materna exclusiva durante los primeros seis meses de vida del bebé es inferior al 1,2\% (247).

Las mujeres que siguen el denominado método lactancia-amenorrea deben tener en cuenta que el riesgo de embarazo aumenta cuando la frecuencia de la lactancia disminuye y por tanto pueden volver a menstruar.

En las seis semanas tras el parto, el riesgo de tromboembolismo venoso es muy elevado, superior al de la mujer embarazada. El riesgo es mucho mayor inmediatamente tras el parto y se modera tras las cuatro semanas, sin estar determinado en qué momento vuelve a la situación basal (245).

Calidad baja

Calidad baja

Calidad baja 
Existen datos consistentes pero con una fiabilidad limitada de que los anticonceptivos de sólo gestágeno no tienen un impacto relevante en el desa- baja rrollo de la lactancia materna o la salud de los bebés (246).

\section{Calidad de la evidencia}

La calidad global de la evidencia es baja.

En la mayoría de aspectos evaluados solamente se dispone de estudios observacionales con diversas limitaciones. Los estudios incluidos en las revisiones de Jackson sobre el retorno a la menstruación y el riesgo de tromboembolismo tras el parto o bien no fueron controlados (243), o bien mostraron un riesgo de sesgo selección y del control de los confusores considerable (245). Los estudios que evaluaron el método MELA eran estudios con un alto riesgo de sesgo de selección (247), y los que evaluaron el impacto de los anticonceptivos de sólo gestágeno mostraron limitaciones en la definición de los desenlaces de interés y no controlaron adecuadamente los confusores de interés (246) además de presentar datos que no permiten conocer el impacto de la exposición a largo plazo (4).

\section{Valores y preferencias de las usuarias}

La Estrategia de Salud Sexual y Reproductiva del SNS identifica el puerperio como un periodo en el que se debe promover la salud de las mujeres, en el que se pueden atender sus necesidades, facilitando el asesoramiento y los cuidados necesarios para su bienestar (1). Por este motivo el puerperio es una oportunidad para ofrecer una visión amplia de la vivencia de la sexualidad, y fomentar espacios de confianza en los que las mujeres y sus parejas puedan abordar sus vivencias respecto a la sexualidad, ofrecer un consejo contraceptivo adecuado y aclarar ideas erróneas sobre la sexualidad tras el embarazo.

\section{Beneficios y riesgos/carga de las intervenciones}

Ver apartado de justificación.

\section{Balance beneficio/riesgo}

Ver apartado de justificación.

\section{Uso de recursos y costes}

Ver apartado de justificación.

\section{Justificación}

Las recomendaciones se han adaptado de la "Guía de Práctica Clínica de Atención en el Embarazo y Puerperio" del programa de Guías de Práctica Clínica en el SNS (242), que a su vez se basan en los criterios de elegibilidad de la OMS. Las recomendaciones en este apartado se han enfocado a promover la información sobre la anticoncepción durante el 
puerperio, remarcando la conveniencia de introducir un método anticonceptivo a partir de los 21 días tras el parto, y ofreciendo la opción del MELA para aquellas mujeres que deciden instaurar la lactancia materna exclusiva. Para la elección de un método anticonceptivo concreto, y la valoración de los riesgos y beneficios en cada situación, se remite a los profesionales a los criterios de elegibilidad.

\section{Recomendaciones}

\begin{tabular}{|l|l|}
\hline $\begin{array}{l}\text { Buena } \\
\text { práctica } \\
\text { clínica }\end{array}$ & $\begin{array}{l}\text { Se sugiere que los profesionales sanitarios promuevan durante el puerperio } \\
\text { espacios en los que se pueda tratar con las mujeres y sus parejas aspectos } \\
\text { relacionados con el consejo contraceptivo y la vivencia de la sexualidad en } \\
\text { esta etapa. }\end{array}$ \\
\hline $\begin{array}{l}\text { Buena } \\
\text { práctica } \\
\text { clínica }\end{array}$ & $\begin{array}{l}\text { Se sugiere consultar los criterios de elegibilidad disponibles para el uso de } \\
\text { anticonceptivos para individualizar la elección del método anticonceptivo } \\
\text { más adecuado de acuerdo con las características y antecedentes de cada mu- } \\
\text { jer. }\end{array}$ \\
\hline Fuerte & $\begin{array}{l}\text { Se recomienda informar sobre la necesidad de anticoncepción y la introduc- } \\
\text { ción de un método adaptado a la situación específica de las mujeres que de- } \\
\text { sean planificar sus futuros embarazos y que no mantienen lactancia materna } \\
\text { exclusiva. }\end{array}$ \\
\hline Débil & $\begin{array}{l}\text { Se sugiere informar sobre la posibilidad de seguir el método lactancia-ame- } \\
\text { norrea (MELA) hasta los 6 meses tras el parto a aquellas mujeres en las que } \\
\text { persiste la amenorrea, realizan lactancia materna exclusiva, y no presentan } \\
\text { riesgo de transmitir o adquirir una infección de transmisión sexual. }\end{array}$ \\
\hline
\end{tabular}





\section{Estrategias diagnósticas y terapéuticas}

\section{Algoritmo 1. Exploraciones y pruebas necesarias antes de la prescripción de anticonceptivos}

\begin{tabular}{|c|c|c|c|c|c|}
\hline & \multicolumn{5}{|c|}{ Método anticonceptivo } \\
\hline & $\begin{array}{c}\text { DIU } \\
\text { (Cu o LNG) }\end{array}$ & Implante & Inyectable & $\begin{array}{c}\text { Píldora } \\
\text { Parche } \\
\text { Anillo }\end{array}$ & $\begin{array}{c}\text { Sólo } \\
\text { gestágeno }\end{array}$ \\
\hline \multicolumn{6}{|l|}{ Exploración } \\
\hline Presión arterial & $\mathrm{C}$ & C & C & $A$ & $\mathrm{C}$ \\
\hline Peso/IMC & $\dagger$ & $\dagger$ & $\dagger$ & $\dagger$ & $\dagger$ \\
\hline Exploración mamaria & $\mathrm{C}$ & C & C & C & $\mathrm{C}$ \\
\hline Examen pélvico & A & C & C & $\mathrm{C}$ & $\mathrm{C}$ \\
\hline \multicolumn{6}{|c|}{ Prueba de laboratorio } \\
\hline Nivel de glucosa & $\mathrm{C}$ & C & C & C & C \\
\hline Lípidos & C & C & C & C & C \\
\hline Función hepática & C & C & C & C & C \\
\hline Hemoglobina & C & C & C & C & C \\
\hline Citología & C & C & C & C & C \\
\hline Serología (ITS) & $\ddagger$ & C & C & C & C \\
\hline Serología $(\mathrm{VIH})$ & $\mathrm{C}$ & C & C & C & $\mathrm{C}$ \\
\hline
\end{tabular}

Abreviaciones: DIU dispositivo intrauterino; Cu cobre; LNG levonorgestrel; IMC índice de masa corporal; ITS infección de transmisión sexual; $\mathrm{VIH}$ virus de la inmunodeficiencia humana.

Las letras representan la clasificación de la utilidad de cada una de las pruebas:

Clase A: obligatoria en todas las ocasiones para un uso eficaz y seguro del anticonceptivo;

Clase B: contribuye de manera sustancial al uso eficaz y seguro del anticonceptivo, pero su implantación debe considerarse en el contexto en que de desea aplicar;

Clase C: no contribuye al uso eficaz y seguro del anticonceptivo.

† La medida del peso (o IMC) no es necesaria para determinar la elegibilidad de un método anticonceptivo, aunque puede ser de ayuda en la primera visita para realizar un seguimiento en futuras consultas.

‡ La mayoría de mujeres no requieren de un cribado especial de ITS en el momento de la inserción de un DIU si ya se les ha realizado el cribado en otros momentos en los que está indicado. Si la mujer requiere de un cribado puede realizarse antes de la inserción, y si no cumple ninguna contraindicación en los criterios de elegibilidad, la inserción no debe retrasarse. En las mujeres con alguna contraindicación para el uso de un DIU, la inserción debe retrasarse hasta disponer de los resultados de la serología y haber tratado a aquellas mujeres con un resultado positivo.

Adaptado de: OMS 2004(88) y CDC 2013(3). 


\section{Algoritmo 2. Actuación ante la alteración del patrón de sangrado en anticoncepción hormonal o intrauterina}

\begin{tabular}{|c|c|c|c|c|c|}
\hline & DIU Cu & DIU LNG & Implante & Inyectable & $\begin{array}{c}\text { Anticonceptivo } \\
\text { hormonal combinado }\end{array}$ \\
\hline $\begin{array}{l}\text { Consideraciones } \\
\text { previas }\end{array}$ & $\begin{array}{l}\text { Antes de la inserción } \\
\text { debe informarse } \\
\text { a la usuaria de la } \\
\text { modificación en su } \\
\text { patrón de sangrado, } \\
\text { y que los manchados } \\
\text { o sangrados no } \\
\text { esperados pueden } \\
\text { ser habituales } \\
\text { entre los } 3 \text { y } 6 \\
\text { primeros meses, } \\
\text { sin entrañar mayor } \\
\text { riesgo y tendiendo a } \\
\text { desaparecer con su } \\
\text { uso continuado. }\end{array}$ & $\begin{array}{l}\text { Antes de la inserción debe } \\
\text { informarse a la usuaria de } \\
\text { la posible modificación en } \\
\text { su patrón de sangrado, } \\
\text { y que los manchados o } \\
\text { sangrados no esperados } \\
\text { pueden ser habituales entre } \\
\text { los } 3 \text { y } 6 \text { primeros meses, } \\
\text { sin entrañar mayor riesgo. } \\
\text { Con el DIU LNG el sangrado } \\
\text { acostumbra a reducirse } \\
\text { con su uso, pudiendo } \\
\text { observarse un sangrado } \\
\text { menstrual leve o amenorrea. } \\
\text { El sangrado frecuente o } \\
\text { prolongado, tanto si no } \\
\text { es esperado como si es } \\
\text { menstrual, es poco común } \\
\text { con su uso. }\end{array}$ & $\begin{array}{l}\text { Antes de la inserción } \\
\text { debe informarse a la } \\
\text { usuaria de la posible } \\
\text { modificación en su } \\
\text { patrón de sangrado, y } \\
\text { que los manchados o } \\
\text { sangrados no esperados } \\
\text { pueden ser habituales, } \\
\text { pudiendo observarse } \\
\text { amenorrea. } \\
\text { Estos cambios en el } \\
\text { patrón de sangrado no } \\
\text { entrañan mayor riesgo. } \\
\text { El sangrado frecuente } \\
\text { o prolongado, tanto si } \\
\text { no es esperado como si } \\
\text { es menstrual, es poco } \\
\text { común con el uso del } \\
\text { implante. }\end{array}$ & $\begin{array}{l}\text { Antes de iniciar el } \\
\text { tratamiento debe informarse } \\
\text { a la usuaria de la posible } \\
\text { modificación en su patrón } \\
\text { de sangrado durante su } \\
\text { uso, y que los manchados } \\
\text { o sangrados no esperados } \\
\text { pueden ser habituales, } \\
\text { pudiendo observarse } \\
\text { amenorrea e incluso algún } \\
\text { sangrado frecuente o } \\
\text { prolongado. Estos cambios } \\
\text { en el patrón de sangrado } \\
\text { no entrañan mayor riesgo, y } \\
\text { deben disminuir con su uso. }\end{array}$ & $\begin{array}{l}\text { Antes de iniciar el tratamiento } \\
\text { debe informarse a la usuaria de la } \\
\text { posible modificación en su patrón } \\
\text { de sangrado durante su uso, y que } \\
\text { los manchados o sangrados no } \\
\text { esperados pueden ser habituales } \\
\text { en los primeros } 3 \text { a } 6 \text { meses. Estos } \\
\text { cambios en el patrón de sangrado } \\
\text { no entrañan mayor riesgo, y deben } \\
\text { disminuir con su uso. }\end{array}$ \\
\hline
\end{tabular}




\section{Actuación ante la alteración del patrón de sangrado en anticoncepción hormonal o intrauterina (cont.)}

\begin{tabular}{|c|c|c|c|c|c|}
\hline & DIU Cu & DIU LNG & Implante & Inyectable & $\begin{array}{c}\text { Anticonceptivo } \\
\text { hormonal combinado }\end{array}$ \\
\hline $\begin{array}{l}\text { Valoración } \\
\text { del patrón de } \\
\text { sangrado }\end{array}$ & $\begin{array}{l}\text { Ante cualquier } \\
\text { sospecha clínica, } \\
\text { se debe descartar } \\
\text { cualquier problema } \\
\text { ginecológico como } \\
\text { una malposición } \\
\text { o expulsión del } \\
\text { DIU, una ITS, un } \\
\text { embarazo o alguna } \\
\text { patología uterina, } \\
\text { especialmente ante } \\
\text { aquellas mujeres } \\
\text { que presentan un } \\
\text { sangrado inesperado } \\
\text { tras haber usado } \\
\text { el DIU durante un } \\
\text { tiempo. En caso } \\
\text { de identificar algún } \\
\text { problema, éste debe } \\
\text { ser tratado. }\end{array}$ & $\begin{array}{l}\text { Ante cualquier sospecha } \\
\text { clínica, se debe descartar } \\
\text { cualquier problema } \\
\text { ginecológico como una } \\
\text { malposición o expulsión del } \\
\text { DIU, una ITS, un embarazo } \\
\text { o alguna patología uterina. } \\
\text { En caso de identificar algún } \\
\text { problema, éste debe ser } \\
\text { tratado. }\end{array}$ & $\begin{array}{l}\text { Ante cualquier sospecha } \\
\text { clínica, se debe } \\
\text { descartar cualquier } \\
\text { problema ginecológico } \\
\text { como una pérdida, } \\
\text { una ITS, un embarazo, } \\
\text { interacción con un } \\
\text { medicamento o alguna } \\
\text { patología uterina. En } \\
\text { caso de identificar algún } \\
\text { problema, éste debe ser } \\
\text { tratado. }\end{array}$ & $\begin{array}{l}\text { Si aparecen sangrados } \\
\text { inesperados de cualquier } \\
\text { tipo, ante cualquier } \\
\text { sospecha clínica, se } \\
\text { debe descartar cualquier } \\
\text { problema ginecológico } \\
\text { como una ITS, un } \\
\text { embarazo, interacción } \\
\text { con un medicamento o } \\
\text { alguna patología uterina. } \\
\text { En caso de identificar algún } \\
\text { problema, éste debe ser } \\
\text { tratado. }\end{array}$ & $\begin{array}{l}\text { Si aparecen sangrados inesperados } \\
\text { de cualquier tipo, ante cualquier } \\
\text { sospecha clínica, se debe descartar } \\
\text { cualquier problema ginecológico } \\
\text { como una ITS, un embarazo, } \\
\text { interacción con un medicamento o } \\
\text { alguna patología uterina. En caso de } \\
\text { identificar algún problema, éste debe } \\
\text { ser tratado. }\end{array}$ \\
\hline
\end{tabular}




\section{Actuación ante la alteración del patrón de sangrado en anticoncepción hormonal o intrauterina (cont.)}

\begin{tabular}{|c|c|c|c|c|c|}
\hline & DIU Cu & DIU LNG & Implante & Inyectable & $\begin{array}{c}\text { Anticonceptivo } \\
\text { hormonal combinado }\end{array}$ \\
\hline $\begin{array}{l}\text { Actuación } \\
\text { en caso de } \\
\text { persistencia } \\
\text { del sangrado, } \\
\text { y ausencia } \\
\text { de problemas } \\
\text { ginecológicos }\end{array}$ & $\begin{array}{l}\text { Considerar el } \\
\text { tratamiento con un } \\
\text { AINE durante } 5 \text { ó } 7 \\
\text { días }\end{array}$ & & $\begin{array}{l}\text { Considerar el } \\
\text { tratamiento con un } \\
\text { AlNE durante } 5 \text { ó } 7 \\
\text { días, o un tratamiento } \\
\text { hormonal (si se cumplen } \\
\text { criterios de elegibilidad) } \\
\text { con anticonceptivos } \\
\text { combinados o } \\
\text { estrógenos en dosis } \\
\text { bajas durante } 10 \text { ó } 20 \\
\text { días. }\end{array}$ & $\begin{array}{l}\text { En caso de sangrados no } \\
\text { esperados o infrecuentes, si } \\
\text { la mujer acepta tratamiento } \\
\text { para el sangrado, y no } \\
\text { existe ningún otro problema } \\
\text { ginecológico, se puede } \\
\text { considerar el tratamiento } \\
\text { con un AINE durante } 5 \text { ó } \\
7 \text { días. Este tratamiento } \\
\text { también puede considerarse } \\
\text { en usuarias con las mismas } \\
\text { condiciones que presentan } \\
\text { un sangrado frecuente } \\
\text { o prolongado, caso en } \\
\text { el que también puede } \\
\text { considerarse un tratamiento } \\
\text { hormonal (si se cumplen } \\
\text { criterios de elegibilidad) con } \\
\text { anticonceptivos combinados } \\
\text { o estrógenos en dosis bajas } \\
\text { durante } 10 \text { ó } 20 \text { días. }\end{array}$ & $\begin{array}{l}\text { En caso de sangrados no esperados } \\
\text { o infrecuentes, si la mujer acepta } \\
\text { tratamiento para el sangrado, y } \\
\text { no existe ningún otro problema } \\
\text { ginecológico, se puede aconsejar } \\
\text { a la usuaria que interrumpa el } \\
\text { tratamiento durante } 3 \text { ó } 4 \text { días } \\
\text { consecutivos. } \\
\text { Este intervalo libre de hormonas } \\
\text { correspondiente a los días de } \\
\text { descanso del anticonceptivo, no } \\
\text { está indicado en los primeros } 21 \\
\text { días de uso del método hormonal } \\
\text { combinado en ciclos extendidos, ni } \\
\text { está indicado realizarlo en más de } \\
\text { una ocasión en el mismo mes, ya } \\
\text { que el anticonceptivo puede reducir } \\
\text { su eficacia. Como alternativa para la } \\
\text { persistencia de sangrado se puede } \\
\text { ofrecer un cambio de dosis de } \\
\text { estrógenos o de tipo de gestágenos. }\end{array}$ \\
\hline $\begin{array}{l}\text { Actuación si } \\
\text { el sangrado } \\
\text { persiste y es } \\
\text { inaceptable para } \\
\text { la mujer }\end{array}$ & \multicolumn{5}{|c|}{ Valorar el uso de otro método anticonceptivo } \\
\hline $\begin{array}{l}\text { Actuación } \\
\text { en caso de } \\
\text { amenorrea }\end{array}$ & & \multicolumn{3}{|c|}{$\begin{array}{l}\text { Informar a la usuaria de que ésta no requiere de tratamiento médico. Si un patrón } \\
\text { regular de sangrado cambia abruptamente a amenorrea, valorar la posibilidad de un } \\
\text { embarazo. Si la amenorrea persiste y es inaceptable para la mujer, se puede valorar el } \\
\text { uso de otro método anticonceptivo. }\end{array}$} & \\
\hline
\end{tabular}




\section{Algoritmo 3. Manejo de los olvidos de píldoras combinadas}

\begin{tabular}{|l|}
\hline Retraso en la toma de \\
una sola píldora: \\
(transcurren menos \\
de 24 horas desde que \\
se hubiera tenido que \\
tomar la píldora) \\
\hline
\end{tabular}

Olvido en la toma de dos o más píldoras de manera consecutiva:

(transcurren 48 horas o más desde que se hubiera tenido que tomar la píldora)
- Tomar la píldora lo antes posible

- Continuar la toma del resto de píldoras de la manera habitual (incluso si deben tomarse dos píldoras en el mismo día)

- No se requieren medidas anticonceptivas adicionales

- No se requiere anticoncepción de urgencia, pero puede considerarse su uso si el olvido se produce al incio del ciclo o durante la última semana del ciclo anterior
- Tomar la píldora lo antes posible, y descartar otros posibles olvidos

- Continuar la toma del resto de píldoras de la manera habitual (incluso si deben tomarse dos píldoras en el mismo día)

- Utilizar un método adicional (de barrera) hasta haber tomado la píldora durante siete días consecutivos

- Si el olvido se ha producido en la última semana de toma de anticonceptivos (antes del periodo libre de anticonceptivos):

- Evitar el periodo libre de anticonceptivos, terminando el envase de anticonceptivos y empezando un nuevo envase al día siguiente

- Si no se puede empezar un nuevo envase inmediatamente, utilizar un método adicional (de barrera) hasta haber tomado la píldora durante siete días consecutivos de un nuevo envase

- Considerar el uso de anticoncepción de urgencia si el olvido se produjo durante la primera semana de toma del anticonceptivo, y se ha mantenido un coito sin protección en los cinco días previos

- Considerar el uso de anticoncepción de urgencia en otras situaciones individuales en las que se considere apropiado 


\section{Algoritmo 4. Manejo de los olvidos de píldoras de solo gestágeno}

Olvido en la toma de una dosis:

(transcurren 3 horas o más desde que se hubiera tenido que tomar la píldora)

Tomar la píldora lo antes posible

- Continuar la toma del resto de píldoras de la manera habitual (una a diario, en el mismo momento del día, incluso si deben tomarse dos píldoras en el mismo día)

- Utilizar un método adicional (por ejemplo, de barrera) o evitar el coito sin protección hasta haber tomado la píldora correctamente, en el momento adecuado, durante dos días consecutivos.

- Considerar la anticoncepción de urgencia si en el periodo del olvido hubo coito sin protección.

Adaptado de: CDC 2013(3). 


\section{Difusión e implementación}

Las GPC son útiles para mejorar la calidad asistencial y los resultados en términos en salud, y para garantizar el acceso a prácticas clínicas con un beneficio apoyado en los resultados de la literatura científica. Uno de los retos actuales es lograr que las personas a las que va dirigida una GPC, adopten sus recomendaciones y las apliquen en su contexto específico. Para lograr este objetivo, es primordial planificar estrategias que vayan más allá de la simple distribución de la información y que apoyen a la población usuaria a mejorar sus conocimientos y habilidad en el tema (diseminación), así como en la identificación de dificultades y fortalezas en el momento de su uso en un contexto específico (implementación).

El plan para difundir la Guía de Práctica Clínica de Anticoncepción Hormonal e Intrauterina incluye las siguientes intervenciones:

- Presentación de la guía por parte de las autoridades sanitarias a los medios de comunicación.

- Presentación de la guía a las direcciones y subdirecciones de Atención Primaria y Atención Hospitalaria de los diferentes Servicios Regionales de Salud.

- Presentación institucional de la guía, en colaboración con el Ministerio de Sanidad, Consumo y Bienestar Social, a las distintas sociedades científicas y profesionales implicadas así como a los colectivos y asociaciones de usuarias interesadas.

- Distribución dirigida y efectiva a los colectivos profesionales implicados (profesionales de la salud que prestan servicio en Hospitales, Centros de Atención Primaria, o Unidades de Atención a la Salud Sexual y Reproductiva) y a las usuarias de anticonceptivos interesadas para facilitar su difusión.

- Presentación interactiva de la guía en los centros de salud y hospitales por parte de líderes locales de opinión.

- Difusión de la guía en formato electrónico en las páginas web del Ministerio de Sanidad, Consumo y Bienestar Social de GuíaSalud y de las sociedades científicas implicadas en el proyecto.

- Publicación de la guía en revistas científicas.

- Presentación de la guía en actividades científicas (jornadas, congresos y reuniones). 



\section{Anexos}

1. Anexo 1. Eficacia de los métodos anticonceptivos hormonales descritos en la literatura científica.

2. Anexo 2. Fármacos que pueden reducir los niveles de hormonas anticonceptivas o la eficacia contraceptiva de los anticonceptivos hormonales.

3. Anexo 3. Fármacos que aumentan la concentración de las hormonas anticonceptivas.

4. Anexo 4. Declaración de Intereses. 


\section{Anexo 1. Eficacia de los métodos anticonceptivos hormonales descritos en la literatura científica}

Resumen de los resultados de los estudios recopilados en la revisión

\begin{tabular}{|c|c|c|c|c|c|c|c|c|c|c|}
\hline \multirow{2}{*}{$\begin{array}{c}\text { Método } \\
\text { (número de estudios) }\end{array}$} & \multicolumn{2}{|c|}{$\begin{array}{l}\text { Índice de Pearl } \\
\text { (rango) }\end{array}$} & \multirow{2}{*}{$\begin{array}{c}\text { Duración de } \\
\text { los estudios } \\
\text { (rango de } \\
\text { meses) }\end{array}$} & \multicolumn{2}{|c|}{$\begin{array}{l}\text { Índice de Pearl al año de uso } \\
\text { (rango) }\end{array}$} & \multicolumn{2}{|c|}{$\begin{array}{l}\text { Tablas de vida } \\
\text { (rango) }\end{array}$} & \multirow{2}{*}{$\begin{array}{c}\text { Duración de los } \\
\text { estudios (rango } \\
\text { de meses) }\end{array}$} & \multicolumn{2}{|c|}{$\begin{array}{c}\text { Tablas de vida al año de } \\
\text { uso (rango) }\end{array}$} \\
\hline & $\begin{array}{l}\text { Uso } \\
\text { ideal }\end{array}$ & Uso habitual & & $\begin{array}{l}\text { Uso } \\
\text { ideal }\end{array}$ & Uso habitual & $\begin{array}{c}\text { Bruto } \\
\text { por } 100 \\
\text { mujeres }\end{array}$ & $\begin{array}{l}\text { Neto } \\
\text { por } 100 \\
\text { mujeres }\end{array}$ & & $\begin{array}{l}\text { Bruto } \\
\text { por } 100 \\
\text { mujeres }\end{array}$ & $\begin{array}{l}\text { Neto por } 100 \\
\text { mujeres }\end{array}$ \\
\hline $\begin{array}{l}\text { Anticonceptivos orales } \\
\text { combinados (47) }\end{array}$ & $\begin{array}{c}0 a \\
1,26\end{array}$ & 0 a 2,18 & 6 a 36 & $\begin{array}{c}0 a \\
1,26\end{array}$ & 0 a 2,18 & 0,2 a 2,3 & 0,1 a 1,5 & 6 a 28 & 0,2 a 2,3 & 0,1 a 1,33 \\
\hline $20 \mu \mathrm{g} E \mathrm{E}$ & $\begin{array}{c}0 a \\
1,26\end{array}$ & 0 a 1,82 & 6 a 36 & $\begin{array}{c}0 a \\
1,26\end{array}$ & 0 a 1,6 & - & - & 6 a 28 & - & - \\
\hline $30 \mu g$ EE & $\begin{array}{c}0 a \\
0,62\end{array}$ & 0 a 1,19 & 6 a 24 & $\begin{array}{c}0 a \\
0,55\end{array}$ & 0 a 1,19 & - & - & 6 a 12 & - & - \\
\hline $\begin{array}{l}\text { Píldora de progestágeno solo } \\
\text { (desogestrel)(1) }\end{array}$ & 0,14 & 0,41 & 12 & 0,14 & 0,41 & - & - & - & - & - \\
\hline Parche transdérmico(3) & $\begin{array}{l}0,59 \\
a \\
0,99\end{array}$ & 0,71 a 1,24 & 6 a 12 & $\begin{array}{l}0,59 \\
a \\
0,99\end{array}$ & 0,71 a 1,24 & - & - & 6 a 12 & - & - \\
\hline Anillo vaginal(3) & $\begin{array}{c}0,31 \\
a \\
0,96\end{array}$ & 0,25 a 1,23 & 12 & $\begin{array}{c}0,31 \\
a \\
0,96\end{array}$ & 0,25 a 1,23 & - & - & 12 & - & - \\
\hline $\begin{array}{l}\text { Implante subcutáneo de } \\
\text { gestágeno (15) }\end{array}$ & 0 & 0 a 0,3 & 24 a 84 & - & 0 a 0,08 & 0 a 1,3 & 0 a 0,8 & 24 a 84 & 0 a 0,2 & 0 \\
\hline Progestágenos inyectables(16) & 0 & 0 & 12 & 0 & 0 & 0 & 0 a 1,1 & 12 a 36 & 0 & 0 a 1,1 \\
\hline DIU de levonorgestrel(9) & - & 0,09 a 0,11 & 60 & - & 0,1 & 0 a 1,1 & 0 a 0,3 & 36 a 84 & 0 a 0,6 & 0 a 0,1 \\
\hline
\end{tabular}


Índice de fallo de los métodos anticonceptivos hormonales de los estudios europeos incluidos en la revisión sistemática

\begin{tabular}{|c|c|c|c|}
\hline $\begin{array}{c}\text { Estudio } \\
\text { (núm. participantes) }\end{array}$ & Anticonceptivo (régimen) & Uso ideal (IC95\%) & $\begin{array}{l}\text { Uso habitual } \\
\text { (IC95\%) }\end{array}$ \\
\hline \multicolumn{4}{|c|}{ Anticonceptivos orales (24 estudios) } \\
\hline $\begin{array}{l}\text { Ahrendt 2006* } \\
\text { (484 / 405) }\end{array}$ & EE30 DRSP3 (21/7) & $\begin{array}{c}0,55 \\
(0,66 \text { a } 1,9)\end{array}$ & \\
\hline Cibula 2006 (516) & EE20 DRSP3 (21/7) & $\begin{array}{c}0,12 \\
\text { (límite sup 0,67) }\end{array}$ & $\begin{array}{c}0,23 \\
\text { (límte sup 0,84) }\end{array}$ \\
\hline Oddsson 2005 (518) & EE30 LNG150 21/7 & $0,53(0,06$ a 1,90$)$ & $1,19$ (0,39 a 2,79$)$ \\
\hline Golbs 2002 (431) & EE30 DNG2 (21/7) & 0 & 0 \\
\hline Golbs 2002 (557) & EE30 DNG2 (21/7) & 0,198 & 0,596 \\
\hline Endrink 2001* (518) & $\begin{array}{l}\text { EE20 GSD75 (23/5) } \\
\text { EE20 GSD150 (23/5) }\end{array}$ & 0 & 0 \\
\hline Ferguson 2000 (2025) & $\begin{array}{l}\text { EE35 DSG50 / EE35 DSG100 / } \\
\text { EE35 DSG150 (21/7) }\end{array}$ & $0,12(0,03$ a 0,70$)$ & $0,25(0,03$ a 0,70$)$ \\
\hline Huber 2000* (1657 / 412) & $\begin{array}{l}\text { EE30 DRSP3 }(21 / 7) \\
\text { EE30 DSG150 }(21 / 7)\end{array}$ & $\begin{array}{l}0,07 \\
0,28\end{array}$ & $\begin{array}{l}0,71 \\
\mathrm{~N} / \mathrm{A}\end{array}$ \\
\hline $\begin{array}{l}\text { Gestodene Study Group } \\
3241999^{*}(539 / 535)\end{array}$ & $\begin{array}{l}\text { EE15 GSD60 }(21 / 7) \\
\text { EE15 DSG150 (21/7) }\end{array}$ & & $\begin{array}{c}0,44 \\
0\end{array}$ \\
\hline $\begin{array}{l}\text { Gestodene Study Group } \\
322 \text { 1999* (1496) }\end{array}$ & EE15 GSD 60 (24/4) & & 0,21 (en 19 ciclos) \\
\hline $\begin{array}{l}\text { Endrik 1999*} \\
(740 / 736)\end{array}$ & $\begin{array}{l}\text { EE20 GSD75 (21/7) } \\
\text { EE20 GSD150 (21/7) }\end{array}$ & & $\begin{array}{l}1,06 \\
0,53\end{array}$ \\
\hline Hite 1999 (12843) & EE20 LNG100 (21/7) & 0,44 & \\
\hline Zahradnik 1998 (1655) & EE30 Chlor acetato2 (21/7) & $0,26(0,019$ a 0,60$)$ & $0,64(0,35$ a 1,09$)$ \\
\hline $\begin{array}{l}\text { Bannmerschult } 1997 \\
\text { (805) }\end{array}$ & EE20 LNG100 (21/7) & 0,29 & \\
\hline Dustenberg 1996 (670) & EE20 GSD75 (21/7) & & 0,07 \\
\hline Brill 1994 (5602) & EE30 DSG150 (21/7) & 0,08 & \\
\hline Bruyniks 1994 (461) & EE30 DSG150 & 0,0 & $0,0(0,0$ a 1,51$)$ \\
\hline $\begin{array}{l}\text { Akerlund } 1993^{\star} \\
(485 / 497)\end{array}$ & $\begin{array}{l}\text { EE20 DSG150 (21/7) } \\
\text { EE30 DSG150 (21/7) }\end{array}$ & $\begin{array}{c}0 \\
0,4(0,04 \text { a } 1,5) \\
\end{array}$ & $\begin{array}{c}0,41(0,04 \text { a } 1,5) \\
0,6(0,1 \text { a } 1,6) \\
\end{array}$ \\
\hline Gauthier 1992 (661) & EE35 NGS & 0,37 & 0,55 \\
\hline $\begin{array}{l}\text { Runnembaum } 1992 \\
\text { (59701) }\end{array}$ & EE35 NGS250 (21/7) & & $0,25(0,19$ a 0,31$)$ \\
\hline Brill 1991 (96000) & EE30 GSD75 (21/7) & 0,062 & \\
\hline
\end{tabular}


Índice de fallo de los métodos anticonceptivos hormonales de los estudios europeos incluidos en la revisión sistemática (cont.)

\begin{tabular}{|c|c|c|c|}
\hline $\begin{array}{c}\text { Estudio } \\
\text { (núm. participantes) }\end{array}$ & Anticonceptivo (régimen) & Uso ideal (IC95\%) & $\begin{array}{l}\text { Uso habitual } \\
\text { (IC95\%) }\end{array}$ \\
\hline Dieben 1991* (882) & $\begin{array}{l}\text { EE40 DSG25 / EE30 DSG125 } \\
(7 / 15)\end{array}$ & $0,0(0,0$ a 0,3$)$ & \\
\hline Lammers $1991^{*}(1684)$ & EE20 DSG150 (21/7) & 0,05 & 0,2 \\
\hline Reiner 1991 (2378) & EE30 GSD75 (21/7) & 0 & 0,21 \\
\hline \multicolumn{4}{|c|}{ Píldora de progestágeno solo (1 estudio) } \\
\hline $\begin{array}{l}\text { Collaborative Study } \\
\text { Group } 1998^{\star}(846)\end{array}$ & DSG75 & $\begin{array}{c}0,14(0,003 \mathrm{a} \\
0,766)\end{array}$ & $\begin{array}{c}0,41(0,085 \mathrm{a} \\
1,204)\end{array}$ \\
\hline \multicolumn{4}{|l|}{ Parches (2 estudios) } \\
\hline Urdl 2005* (846) & Parche transdérmico & $\begin{array}{l}0,66(0,0 \text { a } 1,4) \text { al } \\
\text { año de uso }\end{array}$ & $0,88(0,02$ a 1,74$)$ \\
\hline Smallwood 2001* (1664) & Parche transdérmico & $0,99(0,02$ a 1,96$)$ & $1,24(0,15$ a 2,33$)$ \\
\hline \multicolumn{4}{|l|}{ Anillo (3 estudios) } \\
\hline Ahrendt 2006* (499) & Anillo vaginal $(21 / 7)$ & $\begin{array}{c}0,31 \\
(0,008 \text { a } 1,751)\end{array}$ & $\begin{array}{c}0,25 \\
(0,006 \text { a } 1,363)\end{array}$ \\
\hline Oddsson 2005* (499) & Anillo vaginal $(21 / 7)$ & $0,96(0,20$ a 2,82$)$ & $1,23(0,40$ a 2,86$)$ \\
\hline Roumen 2001* (1145) & Anillo vaginal $(21 / 7)$ & & $0,65(0,24$ a 1,41$)$ \\
\hline \multicolumn{4}{|l|}{ Inyectable ( 1 estudio) } \\
\hline Jain $2004^{\star}(1787)$ & $\begin{array}{l}\text { Inyectable de acetato de } \\
\text { medroxiprogesterona }\end{array}$ & 0 & 0 \\
\hline \multicolumn{2}{|l|}{ DIU LNG (3 estudios) } & $\begin{array}{l}\text { Tablas de vida } \\
\text { (bruto) }\end{array}$ & $\begin{array}{l}\text { Tablas de vida } \\
\text { (neto) }\end{array}$ \\
\hline Backman 2004 (17360) & DIU LNG52mg & $\begin{array}{l}0,1 \text { al año / } \\
0,11 \text { a los } 5 a\end{array}$ & \\
\hline Cox 2002 (678) & DIU LNG52mg & & $\begin{array}{l}0,6 \text { al año } / 1,0 \text { a } \\
\text { los } 2,4 \text { y } 5 \text { años }\end{array}$ \\
\hline Toivonen 1991* (1821) & DIU LNG20 & & 0,3 \\
\hline
\end{tabular}




\section{Anexo 2. Fármacos que pueden reducir los niveles de hormonas anticonceptivas o la eficacia contraceptiva de los anticonceptivos hormonales}

\begin{tabular}{|c|c|c|c|}
\hline Tipo de fármaco & Fármaco & Interacción & Relevancia clínica \\
\hline \multirow[t]{3}{*}{ Antiepilépticos } & $\begin{array}{l}\text { Carbamacepina } \\
\text { Eslicarbamacepina } \\
\text { Oxcarbamacepina } \\
\text { Fenobarbital } \\
\text { Fenitoína } \\
\text { Primidona } \\
\text { Rufinamida }\end{array}$ & Inductor enzimático & $\begin{array}{l}\text { De modesta a marcada } \\
\text { reducción del EE y los } \\
\text { progestágenos. Posible } \\
\text { reducción de la eficacia } \\
\text { contraceptiva. }\end{array}$ \\
\hline & Topiramato & Inductor enzimático débil & $\begin{array}{l}\text { Eficacia contraceptiva puede no } \\
\text { afectarse a dosis terapéuticas } \\
\text { bajas de topiramato. }\end{array}$ \\
\hline & Lamotrigina & $\begin{array}{l}\text { No induce las enzimas } \\
\text { hepáticas }\end{array}$ & $\begin{array}{l}\text { Disminución < del LNG al } \\
\text { administrar como AOC, aunque } \\
\text { evidencia sugiere que la eficacia } \\
\text { de los métodos hormonales } \\
\text { combinados no se ve afectada. } \\
\text { No hay datos sobre el efecto en } \\
\text { métodos de solo progestágenos }\end{array}$ \\
\hline Antiobióticos & $\begin{array}{l}\text { Rifabutina } \\
\text { Rifampicina }\end{array}$ & Inductor enzimático & $\begin{array}{l}\text { Rifabutina asociada a modesta } \\
\text { disminución del EE o estradiol y } \\
\text { progestágenos. Rifampicina tiene } \\
\text { marcados efectos }\end{array}$ \\
\hline \multirow{3}{*}{$\begin{array}{l}\text { Fármacos que } \\
\text { aumentan el pH } \\
\text { gástrico }\end{array}$} & $\begin{array}{l}\text { Inhibidores de } \\
\text { bomba protones }\end{array}$ & Incrementan el PH gástrico & \multirow{3}{*}{$\begin{array}{l}\text { Reducción de las } \\
\text { concentraciones en plasma en } \\
\text { la anticoncepción de urgencia, } \\
\text { (como ulipristal) disminuyendo } \\
\text { su eficacia. No se recomienda el } \\
\text { uso concomitante. }\end{array}$} \\
\hline & Antiácidos & Inductor enzimático & \\
\hline & $\begin{array}{l}\text { Antogonistas de los } \\
\text { receptores } \mathrm{H} 2\end{array}$ & Inductor enzimático & \\
\hline Otros & Sugammadex & $\begin{array}{l}\text { Agente selectivo de unión a } \\
\text { bloqueantes }\end{array}$ & $\begin{array}{l}\text { Interacción hipotética. Modesta } \\
\text { reducción de niveles de AO. } \\
\text { Administrar } 1 \text { dosis en bolo } \\
\text { de sugammadex equivale } \\
\text { a } 1 \text { dosis diaria olvidada de } \\
\text { contraceptivos esteroideos } \\
\text { orales (o combinados o solo } \\
\text { progestágenos). Seguir guía } \\
\text { para píldora olvidada. Para otros } \\
\text { métodos usar precauciones } \\
\text { adicionales durante } 7 \text { días }\end{array}$ \\
\hline
\end{tabular}




\section{Fármacos que pueden reducir los niveles de hormonas anticonceptivas o la eficacia contraceptiva de los anticonceptivos hormonales (cont.)}

\begin{tabular}{|c|c|c|c|}
\hline Tipo de fármaco & Fármaco & Interacción & Relevancia clínica \\
\hline \multirow[t]{4}{*}{$\begin{array}{l}\text { Antiretrovirales } \\
\text { (Inhibidores de } \\
\text { proteasa) }\end{array}$} & $\begin{array}{l}\text { Ritonavir } \\
\text { Ritonavir } \\
\text { potenciado con } \\
\text { saquinavir }\end{array}$ & $\begin{array}{l}\text { Inductor enzimático. Efecto } \\
\text { inhibidor sobre enzima } \\
\text { citocromo P-450. Inductor } \\
\text { de la glucoronidación }\end{array}$ & $\begin{array}{l}\text { Marcada reducción del EE (40\%). } \\
\text { Necesario uso de métodos } \\
\text { contraceptivos adicionales y/o } \\
\text { alternativos }\end{array}$ \\
\hline & $\begin{array}{l}\text { Ritonavir } \\
\text { potenciado con } \\
\text { atazanovir }\end{array}$ & $\begin{array}{l}\text { Atazanovir inhibe } \\
\text { CYP3A4. Combinado } \\
\text { puede predominar la } \\
\text { glucoronidación de ritonavir. }\end{array}$ & $\begin{array}{l}\text { El efecto neto es una reducción } \\
\text { menor del EE y un marcado } \\
\text { incremento en el norgestimato y } \\
\text { el NET-EN. }\end{array}$ \\
\hline & $\begin{array}{l}\text { Ritonavir } \\
\text { potenciado con } \\
\text { tipranavir }\end{array}$ & $\begin{array}{l}\text { Tipranavir (sustrato), } \\
\text { inductor e inhibidor del } \\
\text { citocromo P-450 CYP3A4. } \\
\text { Ritonavir puede tener un } \\
\text { efecto predominante }\end{array}$ & $\begin{array}{l}\text { El efecto neto es una marcada } \\
\text { reducción del EE. }\end{array}$ \\
\hline & $\begin{array}{l}\text { Ritonavir } \\
\text { potenciado con } \\
\text { otros inhibidores de } \\
\text { proteasas* }\end{array}$ & $\begin{array}{l}\text { Puede predominar el efecto } \\
\text { de ritonavir }\end{array}$ & $\begin{array}{l}\text { Reducción del EE y } \\
\text { progestágenos. }\end{array}$ \\
\hline \multirow[t]{2}{*}{$\begin{array}{l}\text { Antiretrovirales } \\
\text { (Inhibidores no } \\
\text { nucleósidos de } \\
\text { transcriptasa } \\
\text { reversa) }\end{array}$} & Efavirenz & Inductor enzimático & $\begin{array}{l}\text { No reduce EE. Si reduce } \\
\text { metabolítos del norgestimato } \\
\text { (norelgestromin y LNG). } \\
\text { Observados algunos embarazos. } \\
\text { No hay una reducción en los } \\
\text { niveles DMPA }\end{array}$ \\
\hline & Nevirapina & Inductor enzimático & $\begin{array}{l}\text { Reducción moderada del EE y } \\
\text { un reducción menor del NET-EN }\end{array}$ \\
\hline \multirow[t]{4}{*}{ Otros } & $\begin{array}{l}\text { Hierba de San } \\
\text { Juan (Hypericum } \\
\text { perforatum) }\end{array}$ & Inductor enzimático & $\begin{array}{l}\text { En un inductor enzimático débil } \\
\text { por lo que potencialmente puede } \\
\text { reducir la eficacia. }\end{array}$ \\
\hline & Bosentán & Inductor enzimático & $\begin{array}{l}\text { Modesta reducción del EE y } \\
\text { progestágeno }\end{array}$ \\
\hline & Modafinilo & Inductor enzimático & Reducción menor del EE \\
\hline & Aprepitant & Inductor enzimático & $\begin{array}{l}\text { Modesta reducción del EE y } \\
\text { progestágeno }\end{array}$ \\
\hline
\end{tabular}




\section{Anexo 3. Fármacos que aumentan la concentración de las hormonas anticonceptivas}

\begin{tabular}{|c|c|c|c|}
\hline Tipo de Fármaco & Fármaco & Interacción & Relevancia clínica \\
\hline Antibacteriano & Eritromicina & $\begin{array}{l}\text { Inhibidor } \\
\text { enzimático }\end{array}$ & $\begin{array}{l}\text { Incremento moderado o importante de } \\
\text { los niveles de estradiol y dienogest. La } \\
\text { relevancia clínica no es clara, sin embargo } \\
\text { se puede prever la incidencia de eventos } \\
\text { adversos. }\end{array}$ \\
\hline Antifúngico & $\begin{array}{l}\text { Fluconazol } \\
\text { Itraconazol } \\
\text { Ketoconazol } \\
\text { Voriconazol }\end{array}$ & $\begin{array}{l}\text { Inhibidor } \\
\text { enzimático }\end{array}$ & $\begin{array}{l}\text { Incremento moderado del EE y los } \\
\text { progestágenos. } \\
\text { Algunos estudios han reportado incidencia } \\
\text { de sangrado. Los estudios que han evaluado } \\
\text { el fluconazol reportan una buena eficacia } \\
\text { anticonceptiva. }\end{array}$ \\
\hline Antiretroviral & Atazanavir (simple) & $\begin{array}{l}\text { Inhibidor } \\
\text { enzimático }\end{array}$ & $\begin{array}{l}\text { Incremento moderado a marcado cuando se } \\
\text { utiliza EE y NET-EN. No se recomienda el uso } \\
\text { concomitante de métodos combinados. }\end{array}$ \\
\hline Inmunosupresor & Tacrolimus & $\begin{array}{l}\text { Inhibidor } \\
\text { enzimático }\end{array}$ & $\begin{array}{l}\text { Teóricamente inhibe el metabolismo de los } \\
\text { estrógenos y progestágenos lo que conlleva } \\
\text { a un aumento de los niveles. La relevancia } \\
\text { clínica no es clara pero el incremento parece } \\
\text { ser pequeño. }\end{array}$ \\
\hline $\begin{array}{l}\text { Antinflamatorios } \\
\text { no esteroides }\end{array}$ & Etoricoxib & $\begin{array}{l}\text { Inhibidor } \\
\text { enzimático }\end{array}$ & $\begin{array}{l}\text { La dosis de etoricoxib igual o mayor a } \\
60 \text { mg incrementa los niveles de EE en } 40 \% \\
\text { o más. Se ha reportado un riesgo potencial } \\
\text { de eventos adversos relacionados a los } \\
\text { estrógenos. }\end{array}$ \\
\hline Estatinas & $\begin{array}{l}\text { Atorvastatin } \\
\text { Rosuvastatin }\end{array}$ & $\begin{array}{l}\text { Inhibidor } \\
\text { enzimático }\end{array}$ & $\begin{array}{l}\text { Incremento moderado en los niveles de EE } \\
\text { y progestágenos. La relevancia clínica no es } \\
\text { clara pero parece ser pequeña. }\end{array}$ \\
\hline Vasodilatadores & Sitaxentan sodium & $\begin{array}{l}\text { Inhibidor } \\
\text { enzimático }\end{array}$ & $\begin{array}{l}\text { Incremento moderado o importante de los } \\
\text { niveles de EE y NET-EN. La relevancia clínica } \\
\text { no es clara pero se pueden anticipar eventos } \\
\text { adversos. }\end{array}$ \\
\hline
\end{tabular}




\section{Anexo 4. Declaración de Intereses. GPC Anticoncepción Hormonal e Intrauterina}

Los siguientes miembros del grupo elaborador han declarado ausencia de intereses:

Lorenzo Arribas Mir, Natividad Fidalgo Rodríguez, Patricia Gavín Benavent, Ivan Solà, Juan Ignacio Martín Sánchez.

$\mathrm{M}^{\mathrm{a}}$ Jesús Alonso Llamazares, ha recibido financiación de Sociedades Científicas para asistir a reuniones, congresos y cursos, ha recibido honorarios de SEFAC en concepto de ponencias, Y ha realizado consultoría para Laboratorio HRA compañía farmacéutica / otras tecnologías.

Joaquim Calaf Alsina, ha recibido financiación del Hospital de la Santa Creu i Sant Pau Servei de Ginecología, para asistir a reuniones, congresos y cursos, Ha recibido honorario como ponente y Financiación por participar en una investigación y consultoría para una compañía farmacéutica / otras tecnologías.

$\mathrm{M}^{\mathrm{a}}$ Jesús Cancelo Hidalgo, ha recibido financiación de Exeltis para asistir a reuniones, congresos y cursos. Y ha recibido honorarios de Bayer, Pfzer, Grünenthal, MSD, Rovi en concepto de ponencias. Ha recibido de Teva, Gedeon Richter, financiación de programas educativos o cursos, ha realizado consultoría para Shionogui compañía farmacéutica u otras tecnologías.

Esther de la Viuda García, ha recibido financiación para asistir a reuniones, congresos y cursos. Y ha recibido honorarios en concepto de ponencias. Ha colaborado con estudios de investigación, coordinación y redacción de libros o capítulos de los mismos, entre otras actividades científicas y docentes con los siguientes laboratorios farmacéuticos relacionados con la ginecología y la anticoncepción: BAYER, MSD, HRA, CHIESI, MERCK, EFFIK y BIAL y forma parte del board nacional de TEVA.

José Vicente González Navarro, ha recibido financiación de Gedeon Richter para asistir a reuniones, congresos y cursos. Y ha recibido honorarios de GSK, MSD, Gedeon Richter, Teva, Exeltix, Sanofi, Effik, Casen, en concepto de ponencias.

Paloma Lobo Abascal, ha recibido honorarios de Bayer, Exeltis, Hra, MSD, TEVA en concepto de ponencias. Ha recibido de MSD financiación de programas educativos o cursos Y ha realizado consultoría para TEVA compañía farmacéutica / otras tecnologías como miembro del Board Europeo.

Cristina Martínez Bueno, ha recibido financiación de Bayer por participar en una investigación

Francisca Martínez San Andrés, ha recibido financiación de Bayer para asistir a reuniones, congresos y cursos, Y ha recibido honorarios de Ferring en concepto de ponencias. Ha recibido financiación de Bayer por participar en una investigación, ha realizado consultoría para Bayer, Kern Pharma compañía farmacéutica / otras tecnologías.

Ezequiel Pérez Campos, ha recibido financiación de HRA Pharma, Exeltis, MSD, TEVA para asistir a reuniones, congresos y cursos, ha realizado consultoría para Exeltis compañía farmacéutica / otras tecnologías 
Isabel Ramírez Polo, ha recibido financiación de Bayer, Leo Pharma, para asistir a reuniones, congresos y cursos, Y ha recibido honorarios de Bayer, MSD, Organon; Effik, Italfarmaco, Gedeón Rischter, Leo Pharma, en concepto de ponencias. Ha recibido de Bayer, MSD, Organon; Effik, Italfarmaco, Gedeón Rischter, TEVA, Leo Pharma, financiación de programas educativos o cursos, ha sido miembro del Comité de Seguridad de medicamentos de Uso Humano en relación con productos anticonceptivos de la Agencia Española de Medicamentos y Productos Sanitarios.

Los siguientes revisores externos han declarado ausencia de intereses:

Eva Adarve Hidalgo, José Gutiérrez Ales, Roberto Lertxundi, Amparo Ortega del Moral, María Lourdes Ortiz Villanueva, Alberto Salamanca Ballesteros, Isabel Serrano Fuster.

Ma Ángeles Gómez Martínez, ha recibido financiación de Exeltis para asistir a reuniones, congresos y cursos. Y ha recibido honorarios de MSD en concepto de ponencias. Y ha realizado consultoría para TEVA compañía farmacéutica / otras tecnologías

Iñaki Lete Lasa, ha recibido honorarios de MSD, TEVA, HRA Pharma y Exeltis en concepto de ponencias, ha realizado consultoría para MSD España, TEVA Internacional, Merck Internacional, HRA Pharma España, Exeltis, Adamed, compañías farmacéuticas / otras tecnologías. Y ayuda económica de Merck Internacinal para la financiación de una investigación en el Hospital Universitario Araba.

Hildegard Mausbach Reisen, ha recibido financiación de diversas industrias para asistir a reuniones, congresos y cursos. Y ha recibido honorarios de MSD en concepto de ponencias

Nicolás Mendoza Ladrón de Guevara, ha recibido financiación de MSD, Angelini, Deka, para asistir a reuniones, congresos y cursos. Y ha recibido honorarios de MSD, Exeltis y Adamed en concepto de ponencias. Ha realizado Consultoría para Adamed. Y Ha recibido financiación de Shionogi, Puleva, por participar en una investigación.

Ana Felisa Muñoz Ruiz, ha recibido financiación de Bayer, MSD para asistir a reuniones, congresos y cursos. Ha recibido financiación de Sociedad Aragonesa de Anticoncepción, como beca parcial en Máster de Anticoncepción y Salud Sexual. U. Alcalá de H. Y ha recibido honorarios de Sociedad Española de Anticoncepción (Zaragoza), MSD, Colegio Oficial de Enfermería de Zaragoza, SALUD, Servicio Aragonés de la Salud, en concepto de ponencias. Ha recibido financiación de Bayer por participar en una investigación, ha realizado consultoría para $P \& G$ compañía farmacéutica / otras tecnologías.

Núria Parera Junyent, ha recibido financiación de MSD, Teva, Bayer para asistir a reuniones, congresos y cursos, Y ha recibido honorarios de MSD, Teva, en concepto de ponencias, Ha recibido financiación de Bayer por participar en una investigación, ha realizado consultoría para $P \& G$ compañía farmacéutica / otras tecnologías.

Macarena Quesada Moreno, ha recibido financiación de Bayer, Procare Health, para asistir a reuniones, congresos y cursos, Y ha recibido honorarios de MSD en concepto de ponencias, Ha recibido de Janssen, Gynea, MSD, Gedeon Richter, MSD, Sandoz, Sanofi Pasteur MSD, para financiación de programas educativos o cursos. Ha recibido financiación de Centro de Epidemiología e Investigación de la Salud de Berlín (ZEG) y Kantar 
Health SA, y Bayer, por participar en una investigación. Y ha realizado consultoría para Effik compañía farmacéutica / otras tecnologías. Otros conflictos de interés: 1. Artículo HPV revista práctica ginecología y obstetricia. Verrugas genitales en la consulta de ginecología, Laboratorios Bial 2015. 2. Update. Anticoncepción e ITS, Laboratorios Pfizer 2016. 


\section{Bibliografía}

1. Ministerio de Sanidad, Política Social e Igualdad. Estrategia Nacional de Salud Sexual y Reproductiva. Madrid: Ministerio de Sanidad, Política Social e Igualdad, 2011.

2. Ministerio de Sanidad y Política Social. Encuesta Nacional de Salud Sexual. Centro de Investigaciones Sociológicas, número 2780, 2009.

3. Centers for Disease Control and Prevention (CDC). U.S. Selected Practice Recommendations for Contraceptive Use, 2013: adapted from the World Health Organization selected practice recommendations for contraceptive use, 2nd edition. MMWR Recomm Rep. 2013;62 (RR-05):1-60.

4. World Health Organization. Medical eligibility criteria for contraceptive use - 5th ed. Genève: World Health Organization 2015.

5. Faculty of Sexual and Reproductive Healthcare (FSRH). UK Medical Eligibility Criteria for Contraceptive Use (2009)

6. Grupo de trabajo para la actualización del Manual de Elaboración de GPC. Elaboración de Guías de Práctica Clínica en el Sistema Nacional de Salud. Actualización del Manual Metodológico [Internet]. Madrid: Ministerio de Sanidad, Servicios Sociales e Igualdad; Zaragoza: Instituto Aragonés de Ciencias de la Salud (IACS); 2016 [acceso en agosto 2016]. Disponible en: http://portal.guiasalud.es/emanuales/elaboracion_2/.

7. Schünemann H, Brożek J, Guyatt G, Oxman A, editores. GRADE handbook for grading quality of evidence and strength of recommendations. Updated October 2013. The GRADE Working Group, 2013. Disponible en línea www.guidelinedevelopment.org/handbook.

8. Sociedad Española de Contracepción. Actualización en el manejo clínico de la anticoncepción hormonal, intrauterina y de urgencia. Conferencia de Consenso. 2011. Disponible online en http://sec.es/area-cientifica/documentacion-cientifica/conferencia-de-consenso/

9. Alonso-Coello P, Rigau D, Solà I, Martínez García L. Formulating health care recommendations: the GRADE system. Med Clin (Barc). 2013;140(8):366-73.

10. Mansour D, Inki P, Gemzell-Danielsson K. Efficacy of contraceptive methods: A review of the literature. Eur J Contracept Reprod Health Care. 2010;15(1):4-16.

11. Wiegratz I, Thaler CJ. Hormonal contraception--what kind, when, and for whom?. Dtsch Arztebl Int. 2011;108(28-29):495-505.

12. Trussell J. Contraceptive failure in the United States. Contraception. 2011;83(5):397-404.

13. Kost K, Singh S, Vaughan B, Trussell J, Bankole A. Estimates of contraceptive failure from the 2002 National Survey of Family Growth. Contraception. 2008;77(1):10-21.

14. Dinger J, Minh TD, Buttmann N, Bardenheuer K. Effectiveness of oral contraceptive pills in a large U.S. cohort comparing progestogen and regimen. Obstet Gynecol. 2011;117(1):33-40.

15. Dinger JC, Heinemann LA, Kühl-Habich D. The safety of a drospirenone-containing oral contraceptive: final results from the European Active Surveillance Study on oral contraceptives based on 142,475 women-years of observation. Contraception 2007;75(5):344-54.

16. Dinger JC, Cronin M, Möhner S, Schellschmidt I, Minh TD, Westhoff C. Oral contraceptive effectiveness according to body mass index, weight, age, and other factors. Am J Obstet Gynecol. 2009;201(3):263.e1-9.

17. Winner B, Peipert JF, Zhao Q, Buckel C, Madden T, Allsworth JE, et al. Effectiveness of long-acting reversible contraception. N Engl J Med. 2012;366(21):1998-2007.

18. Trussell J. Contraceptive efficacy. En: Hatcher RA, Trussell J, Stewart F, editores. Contraceptive technology (18a edición revisada). New York: Ardent Media, 2004. 
19. Gallo MF, Nanda K, Grimes DA, Lopez LM, Schulz KF. $20 \mu \mathrm{g}$ versus $>20 \mu \mathrm{g}$ estrogen combined oral contraceptives for contraception. Cochrane Database of Systematic Reviews 2013, Issue 8. Art. No.: CD003989. DOI: 10.1002/14651858.CD003989.pub5.

20. Anttila L, Kunz M, Marr J. Bleeding pattern with drospirenone $3 \mathrm{mg}+$ ethinyl estradiol 20 mcg 24/4 combined oral contraceptive compared with desogestrel $150 \mathrm{mcg}+\mathrm{ethinyl}$ estradiol $20 \mathrm{mcg}$ 21/7 combined oral contraceptive. Contraception. 2009;80(5):445-51.

21. Gestodene Study Group. Cycle control, safety and efficacy of a 24-day regimen of gestodene $60 \mathrm{microg} /$ ethinylestradiol $15 \mathrm{microg}$ and a 21-day regimen of desogestrel $150 \mathrm{microg} / \mathrm{ethin}$ ylestradiol 20 microg. Gestodene Study Group 324. Eur J Contracept Reprod Health Care. 1999;4 Suppl 2:17-25.

22. Kaunitz AM, Burkman RT, Fisher AC, Laguardia KD. Cycle control with a 21-day compared with a 24-day oral contraceptive pill: a randomized controlled trial. Obstet Gynecol. 2009;114(6):1205-12.

23. Mansour D, Verhoeven C, Sommer W, Weisberg E, Taneepanichskul S, Melis GB, et al.. Efficacy and tolerability of a monophasic combined oral contraceptive containing nomegestrol acetate and $17 \beta$-oestradiol in a $24 / 4$ regimen, in comparison to an oral contraceptive containing ethinylestradiol and drospirenone in a 21/7 regimen. Eur J Contracept Reprod Health Care. 2011;16(6):430-43.

24. Nakajima ST, Archer DF, Ellman H. Efficacy and safety of a new 24-day oral contraceptive regimen of norethindrone acetate $1 \mathrm{mg} /$ ethinyl estradiol 20 micro g (Loestrin $24 \mathrm{Fe}$ ). Contraception. 2007;75(1):16-22.

25. Westhoff C, Kaunitz AM, Korver T, Sommer W, Bahamondes L, Darney P, et al. Efficacy, safety, and tolerability of a monophasic oral contraceptive containing nomegestrol acetate and 17ß-estradiol: a randomized controlled trial. Obstet Gynecol. 2012;119(5):989-99.

26. Dinger J, Bardenheuer K, Heinemann K. Cardiovascular and general safety of a 24-day regimen of drospirenone-containing combined oral contraceptives: final results from the International Active Surveillance Study of Women Taking Oral Contraceptives. Contraception. 2014;89(4):253-63.

27. Mantha S, Karp R, Raghavan V, Terrin N, Bauer KA, Zwicker JI. Assessing the risk of venous thromboembolic events in women taking progestin-only contraception: a meta-analysis. BMJ. 2012;345:e4944. doi: 10.1136/bmj.e4944.

28. Martínez F, Ramírez I, Pérez-Campos E, Latorre K, Lete I. Venous and pulmonary thromboembolism and combined hormonal contraceptives. Systematic review and meta-analysis. Eur J Contracept Reprod Health Care. 2012;17(1):7-29. doi: 10.3109/13625187.2011.643836.

29. Peragallo Urrutia R, Coeytaux RR, McBroom AJ, Gierisch JM, Havrilesky LJ, Moorman PG, et al. Risk of acute thromboembolic events with oral contraceptive use: a systematic review and meta-analysis. Obstet Gynecol. 2013;122(2 Pt 1):380-9. doi: 10.1097/ AOG.0b013e3182994c43.

30. Stegeman BH, de Bastos M, Rosendaal FR, van Hylckama Vlieg A, Helmerhorst FM, Stijnen $\mathrm{T}$, et al. Different combined oral contraceptives and the risk of venous thrombosis: systematic review and network meta-analysis. BMJ. 2013;347:f5298. doi: 10.1136/bmj.f5298.

31. de Bastos M, Stegeman BH, Rosendaal FR, Van Hylckama Vlieg A, Helmerhorst FM, Stijnen T, et al. Combined oral contraceptives: venous thrombosis. Cochrane Database of Systematic Reviews 2014, Issue 3. Art. No.: CD010813. DOI: 10.1002/14651858.CD010813.pub2.

32. Lidegaard O, Nielsen H, Skovlund CW, Skjeldestad FE, Løkkegaard E. Risk of venous thromboembolism from use of oral contraceptives containing different progestogens and oestrogen doses: Danish cohort study, 2001-9. BMJ 2011; 343:d6423.

33. Samuelsson E, Hagg S. Incidence of venous thromboembolism in young Swedish women and possibly preventable cases among combined oral contraceptive users. Acta Obstet Gynecol Scand 2004;83:674-81. 
34. Lidegaard $\varnothing$, Løkkegaard E, Jensen A, Skovlund CW, Keiding N. Thrombotic stroke and myocardial infarction with hormonal contraception. N Engl J Med. 2012;366(24):2257-66.

35. Dinger J, Möhner S, Heinemann K. Cardiovascular risk associated with the use of an etonogestrel-containing vaginal ring. Obstet Gynecol. 2013;122(4):800-8.

36. Agencia Española de Medicamentos y Productos Sanitarios (AEMPS). Anticonceptivos hormonales combinados: conclusiones de la revisión del riesgo de tromboembolismo venoso. Información para profesionales sanitarios. Referencia: MUH (FV), 27/2013.

37. Wyatt KD, Anderson RT, Creedon D, Montori VM, Bachman J, et al. Women's values in contraceptive choice: a systematic review of relevant attributes included in decision aids. BMC Womens Health. 2014;14(1):28. doi: 10.1186/1472-6874-14-28.

38. Garbers S, Meserve A, Kottke M, Hatcher R, Ventura A, Chiasson MA. Randomized controlled trial of a computer-based module to improve contraceptive method choice. Contraception. 2012;86(4):383-90.

39. Lopez LM, Steiner M, Grimes DA, Hilgenberg D, Schulz KF. Strategies for communicating contraceptive effectiveness. Cochrane Database of Systematic Reviews 2013, Issue 4. Art. No.: CD006964. DOI: 10.1002/14651858.CD006964.pub3.

40. Ortmeier B G, Sauer K A, Langley P C, Bealmear B K. A cost-benefit analysis of four hormonal contraceptive methods. Clinical Therapeutics 1994;16(4):707-713.

41. Trussell J, Leveque J A, Koenig J D, London R, Borden S, Henneberry J, et al. The economic value of contraception: a comparison of 15 methods. American Journal of Public Health 1995;85(4):494-503.

42. Sonnenberg F A, Burkman R T, Hagerty C G, Speroff L, Speroff T. Costs and net health effects of contraceptive methods. Contraception 2004;69(6):447-459.

43. Mavranezouli I; LARC Guideline Development Group. The cost-effectiveness of long-acting reversible contraceptive methods in the UK: analysis based on a decision-analytic model developed for a National Institute for Health and Clinical Excellence (NICE) clinical practice guideline. Hum Reprod. 2008;23(6):1338-45.

44. Trussell J, Lalla AM, Doan QV, Reyes E, Pinto L, Gricar J. Cost effectiveness of contraceptives in the United States. Contraception. 2009;79(1):5-14.

45. Cheng L, Che Y, Gülmezoglu AM. Interventions for emergency contraception. Cochrane Database of Systematic Reviews 2012, Issue 8. Art. No.: CD001324. DOI: 10.1002/14651858. CD001324.pub4.

46. Glasier AF, Cameron ST, Fine PM, Logan SJ, Casale W, Van Horn J, et al. Ulipristal acetate versus levonorgestrel for emergency contraception: a randomised non-inferiority trial and meta-analysis. Lancet. 2010;375(9714):555-62.

47. Creinin MD, Schlaff W, Archer DF, Wan L, Frezieres R, Thomas M, et al. Progesterone receptor modulator for emergency contraception: a randomized controlled trial. Obstet Gynecol. 2006;108(5):1089-97.

48. Askalani AH, Al-Senity AM, Al-Agizy HM, Salam HI, Al-Masry GI, El-Sadek SM. Evaluation of copper T-200 as a post-coital contraceptive. Egyptian Journal of Obstetrics and Gynaecology 1987;13:63-6.

49. Liu L, Chen A. A comparative study of mifepristone with Cu-IUD for emergency contraception. Journal of Changzhi Medical College 2002;61:198-9.

50. Cleland K, Zhu H, Goldstuck N, Cheng L, Trussell J. The efficacy of intrauterine devices for emergency contraception: a systematic review of 35 years of experience. Hum Reprod. 2012;27(7):1994-2000.

51. Faculty of Family Planning and Reproductive Health Care. Emergency Contraception - Clinical Effectiveness Unit, August 2011. [accesible online en www.fsrh.org/pdfs/CEUguidanceEmergencyContraception11.pdf, accedido 1 septiembre 2014]. 
52. European Consortium for Emergency Contraception. Emergency Contraception: A guideline for service provision in Europe. ECEC, December 2013. [acceso online en www.ec-ec.org/ emergency-contraception-in-europe/guidelines/, accedido enero 2016].

53. National Institute for Health and Care Excellence. Contraception. NICE Quality Standard [QS129]. London, September 2016.

54. Agencia Española del Medicamento y Productos Sanitarios. Mirena 0,02 mg cada 24 horas sistema de liberación intrauterino. Ficha Técnica o Resumen de las Características del Producto. Disponible en http://www.aemps.gob.es/cima/pdfs/es/ft/63158/FT_63158.pdf

55. North East Treatment Advisory Group. Ulipristal (ellaOne) for post-coital contraception. North East Treatment Advisory Group, September 2009.

56. Agencia Española del Medicamento y Productos Sanitarios. ellaOne $30 \mathrm{mg}$ comprimido (acetato de ulipristal). Ficha Técnica o Resumen de las Características del Producto. Disponible en www.ema.europa.eu/docs/es_ES/document_library/EPAR_Product_Information/human/ 001027/WC500023670.pdf

57. Faculty of Family Planning and Reproductive Health Care. Statement on Drug Interactions between Hormonal Contraception and Ulipristal Products: ellaOne ${ }^{\circledR}$ and Esmya ${ }^{\circledR}$ - Clinical Effectiveness Unit, November 2012. [accesible online en www.fsrh.org/pdfs/CEUstatementEsmya.pdf, accedido 1 septiembre 2013].

58. Polis CB, Grimes DA, Schaffer K, Blanchard K, Glasier A, Harper C. Advance provision of emergency contraception for pregnancy prevention. Cochrane Database of Systematic Reviews 2007, Issue 2. Art. No.: CD005497. DOI: 10.1002/14651858.CD005497.pub2.

59. Ekstrand M, Larsson M, Darj E, Tydén T. Advance provision of emergency contraceptive pills reduces treatment delay: a randomised controlled trial among Swedish teenage girls. Acta Obstet Gynecol Scand. 2008;87(3):354-9.

60. Gold MA, Wolford JE, Smith KA, Parker AM. The effects of advance provision of emergency contraception on adolescent women's sexual and contraceptive behaviors. J Pediatr Adolesc Gynecol. 2004;17(2):87-96.

61. Raine TR, Harper CC, Rocca CH, Fischer R, Padian N, Klausner JD, et al. Direct access to emergency contraception through pharmacies and effect on unintended pregnancy and STIs: a randomized controlled trial. JAMA. 2005;293(1):54-62.

62. Raymond EG, Stewart F, Weaver M, Monteith C, Van Der Pol B. Randomized trial to evaluate the impact of increased access to emergency contraceptive pills. Obstetrics and Gynecology 2006;108(5):1098-106.

63. Meyer JL, Gold MA, Haggerty CL. Advance provision of emergency contraception among adolescent and young adult women: a systematic review of literature. J Pediatr Adolesc Gynecol. 2011 Feb;24(1):2-9.

64. Rodriguez MI, Curtis KM, Gaffield ML, Jackson E, Kapp N. Advance supply of emergency contraception: a systematic review. Contraception. 2013;87(5):590-601.

65. Bayer LL, Edelman AB, Caughey AB, Rodriguez MI. The price of emergency contraception in the United States: what is the cost-effectiveness of ulipristal acetate versus single-dose levonorgestrel? Contraception 2013; 87(3): 385-390.

66. Tepper NK, Curtis KM, Steenland MW, Marchbanks PA. Physical examination prior to initiating hormonal contraception: a systematic review. Contraception. 2013;87(5):650-4.

67. Tepper NK, Curtis KM, Steenland MW, Marchbanks PA. Blood pressure measurement prior to initiating hormonal contraception: a systematic review. Contraception. 2013;87(5):631-8.

68. Tepper NK, Steenland MW, Marchbanks PA, Curtis KM. Laboratory screening prior to initiating contraception: a systematic review. Contraception. 2013;87(5):645-9. 
69. WHO Collaborative Study of Cardiovascular Disease and Steroid Hormone Contraception. Acute myocardial infarction and combined oral contraceptives: results of an international multicentre case-control study. Lancet 1997;349(9060):1202-9.

70. Lewis MA, Heinemann LA, Spitzer WO, MacRae KD, Bruppacher R. The use of oral contraceptives and the occurrence of acute myocardial infarction in young women. Results from the Transnational Study on Oral Contraceptives and the Health of Young Women. Contraception. 1997;56(3):129-40.

71. Dunn N, Thorogood M, Faragher B, de Caestecker L, MacDonald TM, McCollum C, et al. Oral contraceptives and myocardial infarction: results of the MICA case-control study. BMJ. 1999;318(7198):1579-83.

72. Hamilton T, Loudon NB, Prescott RJ, Rankin ME. Detection of breast disease in a family planning association clinic. Scott Med J. 1976;21(1):31-6.

73. Kösters JP, Gøtzsche PC. Regular self-examination or clinical examination for early detection of breast cancer. Cochrane Database of Systematic Reviews 2003, Issue 2. Art. No.: CD003373. DOI: 10.1002/14651858.CD003373.

74. Armstrong KA, Stover MA. SMART START: an option for adolescents to delay the pelvic examination and blood work in family planning clinics. J Adolesc Health. 1994;15(5):389-95.

75. Sawaya GF, Harper C, Balistreri E, Boggess J, Darney P. Cervical neoplasia risk in women provided hormonal contraception without a Pap smear. Contraception. 2001;63(2):57-60.

76. Kahn HS, Curtis KM, Marchbanks PA. Effects of injectable or implantable progestin-only contraceptives on insulin-glucose metabolism and diabetes risk. Diabetes Care. 2003;26(1): 216-25.

77. Diab KM, Zaki MM. Contraception in diabetic women: comparative metabolic study of Norplant, depot medroxyprogesterone acetate, low dose oral contraceptive pill and CuT380A. J Obstet Gynaecol Res. 2000;26(1):17-26.

78. López LM, Grimes DA, Schulz KF. Steroidal contraceptives: effect on carbohydrate metabolism in women without diabetes mellitus. Cochrane Database of Systematic Reviews 2014, Issue 4. Art. No.: CD006133. DOI: 10.1002/14651858.CD006133.pub5.

79. Berenson $\mathrm{AB}$, Rahman $\mathrm{M}$, Wilkinson G. Effect of injectable and oral contraceptives on serum lipids. Obstet Gynecol. 2009;114(4):786-94.

80. Kapp N, Tilley IB, Curtis KM. The effects of hormonal contraceptive use among women with viral hepatitis or cirrhosis of the liver: a systematic review. Contraception. 2009;80(4):381-6.

81. Kapp N, Curtis KM. Hormonal contraceptive use among women with liver tumors: a systematic review. Contraception. 2009;80(4):387-90.

82. International Agency for Research on Cancer. European guidelines for quality assurance in cervical cancer screening (second edition). Lyon: International Agency for Research on Cancer, 2008.

83. International Agency for Research on Cancer. IARC Handbooks of Cancer Prevention, volume 10: Cervix Cancer Screening. Lyon: International Agency for Research on Cancer, 2005.

84. Smith JS, Green J, Berrington de Gonzalez A, Appleby P, Peto J, Plummer M, Franceschi S, Beral V. Cervical cancer and use of hormonal contraceptives: a systematic review. Lancet. 2003 5;361(9364):1159-67.

85. Puig-Tintoré LM, Castellsagué X, Torné A, de Sanjosé S, Cortés J, Roura E, et al. Coverage and factors associated with cervical cancer screening: results from the AFRODITA study: a population-based survey in Spain. J Low Genit Tract Dis. 2008;12(2):82-9.

86. Mohllajee AP, Curtis KM, Peterson HB. Does insertion and use of an intrauterine device increase the risk of pelvic inflammatory disease among women with sexually transmitted infection? A systematic review. Contraception. 2006;73(2):145-53. 
87. Martínez F, López-Arregui E. Infection risk and intrauterine devices. Acta Obstet Gynecol Scand. 2009;88(3):246-50.

88. World Health Organization. Selected practice recommendations for contraceptive use - 2nd ed. Genève: World Health Organization 2004.

89. Faculty of Family Planning and Reproductive Health Care. Combined Hormonal Contraception. Faculty of Sexual \& Reproductive Healthcare Clinical Guidance- Clinical Effectiveness Unit, October 2011 [acceso online en www.fsrh.org/pdfs/CEUGuidanceCombinedHormonalContraception.pdf]

90. Steenland MW, Zapata LB, Brahmi D, Marchbanks PA, Curtis KM. The effect of follow-up visits or contacts after contraceptive initiation on method continuation and correct use. Contraception. 2013;87(5):625-30.

91. Neuteboom K, de Kroon CD, Dersjant-Roorda M, Jansen FW. Follow-up visits after IUDinsertion: sense or nonsense? A technology assessment study to analyze the effectiveness of follow-up visits after IUD insertion. Contraception. 2003;68(2):101-4.

92. Bang S. Korea: the relationship between IUD retention and check-up visits. Stud Fam Plann. 1971;2(5):110-2.

93. Herceg-Baron R, Furstenberg FF Jr, Shea J, Harris KM. Supporting teenagers' use of contraceptives: a comparison of clinic services. Fam Plann Perspect. 1986;18(2):61-6.

94. Kirby D, Raine T, Thrush G, Yuen C, Sokoloff A, Potter SC. Impact of an intervention to improve contraceptive use through follow-up phone calls to female adolescent clinic patients. Perspect Sex Reprod Health. 2010;42(4):251-7.

95. Steenland MW, Zapata LB, Brahmi D, Marchbanks PA, Curtis KM. Appropriate follow up to detect potential adverse events after initiation of select contraceptive methods: a systematic review. Contraception. 2013;87(5):611-24.

96. Cardoso F, Polónia J, Santos A, Silva-Carvalho J, Ferreira-de-Almeida J. Low-dose oral contraceptives and 24-hour ambulatory blood pressure. Int J Gynaecol Obstet. 1997;59(3): 237-43.

97. Coney P, Washenik K, Langley RG, DiGiovanna JJ, Harrison DD. Weight change and adverse event incidence with a low-dose oral contraceptive: two randomized, placebo-controlled trials. Contraception. 2001;63(6):297-302.

98. Review Manager (RevMan) [Computer program]. Version 5.3. Copenhagen: The Nordic Cochrane Centre, The Cochrane Collaboration, 2014.

99. Wilson ES, Cruickshank J, McMaster M, Weir RJ. A prospective controlled study of the effect on blood pressure of contraceptive preparations containing different types and dosages of progestagen. Br J Obstet Gynaecol. 1984;91(12):1254-60.

100. Fasce E, Ibáñez P, Nieto C. Estudio controlado del efecto de anticonceptivos orales sobre la presion arterial. Rev Med Chil. 1995;123(2):158-64.

101. Shen Q, Lin D, Jiang X, Li H, Zhang Z. Blood pressure changes and hormonal contraceptives. Contraception. 1994;50(2):131-41.

102. Farley TM, Rosenberg MJ, Rowe PJ, Chen JH, Meirik O. Intrauterine devices and pelvic inflammatory disease: an international perspective. Lancet. 1992;339(8796):785-8.

103. Sivin I, Alvarez F, Diaz J, Diaz S, el Mahgoub S, Coutinho E, et al. Intrauterine contraception with copper and with levonorgestrel: a randomized study of the TCu $380 \mathrm{Ag}$ and levonorgestrel $20 \mathrm{mcg} /$ day devices. Contraception. 1984;30(5):443-56.

104. Feldblum PJ, Caraway J, Bahamondes L, El-Shafei M, Quan Ha D, Morales E, et al. Randomized assignment to copper IUD or depot-medroxyprogesterone acetate: feasibility of enrollment, continuation and disease ascertainment. Contraception. 2005;72(3):187-91. 
105. Sivin I, Stern J, Diaz J, Diaz MM, Faundes A, el Mahgoub S, et al. Two years of intrauterine contraception with levonorgestrel and with copper: a randomized comparison of the $\mathrm{TCu}$ 380Ag and levonorgestrel $20 \mathrm{mcg} /$ day devices. Contraception. 1987;35(3):245-55.

106. Sivin I, Stern J, Coutinho E, Mattos CE, el Mahgoub S, Diaz S, et al. Prolonged intrauterine contraception: a seven-year randomized study of the levonorgestrel $20 \mathrm{mcg} / \mathrm{day}(\mathrm{LNg} 20)$ and the Copper T380 Ag IUDS. Contraception. 1991;44(5):473-80.

107. Le YC, Rahman M, Berenson AB. Early weight gain predicting later weight gain among depot medroxyprogesterone acetate users. Obstet Gynecol. 2009;114(2 Pt 1):279-84.

108. Bonny AE, Secic M, Cromer B. Early weight gain related to later weight gain in adolescents on depot medroxyprogesterone acetate. Obstet Gynecol. 2011;117(4):793-7.

109. Madden T, Secura GM, Nease RF, Politi MC, Peipert JF. The role of contraceptive attributes in women's contraceptive decision making. Am J Obstet Gynecol. 2015. pii: S0002-9378(15) 00107-6. doi: 10.1016/j.ajog.2015.01.051.

110. Belsey EM. The association between vaginal bleeding patterns and reasons for discontinuation of contraceptive use. Contraception. 1988;38(2):207-25.

111. Faculty of Family Planning and Reproductive Health Care. Management of Unscheduled Bleeding in Women Using Hormonal Contraception. Faculty of Sexual and Reproductive Healthcare - Clinical Effectiveness Unit, May 2009. [accesible online en www.fsrh.org/pdfs/ UnscheduledBleedingMay09.pdf, accedido septiembre 2014].

112. Godfrey EM, Whiteman MK, Curtis KM. Treatment of unscheduled bleeding in women using extended or continuous-use combined hormonal contraception: a systematic review. Contraception. 2013;87(5):567-75.

113. Sulak PJ, Kuehl TJ, Coffee A, Willis S. Prospective analysis of occurrence and management of breakthrough bleeding during an extended oral contraceptive regimen. Am J Obstet Gynecol. 2006;195(4):935-41.

114. Sulak PJ, Smith V, Coffee A, Witt I, Kuehl AL, Kuehl TJ. Frequency and management of breakthrough bleeding with continuous use of the transvaginal contraceptive ring: a randomized controlled trial. Obstet Gynecol. 2008;112(3):563-71.

115. Kaneshiro B, Edelman A, Carlson N, Morgan K, Nichols M, Jensen J. Treatment of unscheduled bleeding in continuous oral contraceptive users with doxycycline: a randomized controlled trial. Obstet Gynecol. 2010;115(6):1141-9.

116. Abdel-Aleem H, d'Arcangues C, Vogelsong KM, Gaffield ML, Gülmezoglu AM. Treatment of vaginal bleeding irregularities induced by progestin only contraceptives. Cochrane Database of Systematic Reviews 2013, Issue 10. Art. No.: CD003449. DOI: 10.1002/14651858. CD003449.pub5.

117. Godfrey EM, Folger SG, Jeng G, Jamieson DJ, Curtis KM. Treatment of bleeding irregularities in women with copper-containing IUDs: a systematic review. Contraception. 2013;87(5): 549-66.

118. Grimes DA, Hubacher D, Lopez LM, Schulz KF. Non-steroidal anti-inflammatory drugs for heavy bleeding or pain associated with intrauterine-device use. Cochrane Database of Systematic Reviews 2006, Issue 4. Art. No.: CD006034. DOI: 10.1002/14651858.CD006034.pub2.

119. Zapata LB, Steenland MW, Brahmi D, Marchbanks PA, Curtis KM. Effect of missed combined hormonal contraceptives on contraceptive effectiveness: a systematic review. Contraception. 2013;87(5):685-700.

120. Mansour D. Revision of the 'missed pill' rules. J Fam Plann Reprod Health Care. 2011;37(3): 128-31.

121. Faculty of Family Planning and Reproductive Health Care. Missed Pill Recommendations. CEU Statement (May 2011). Faculty of Sexual and Reproductive Healthcare - Clinical Effectiveness Unit, May 2011. [accesible online en www.fsrh.org/pdfs/CEUStatementMissedPills. pdf, accedido septiembre 2014]. 
122. Halpern V, Lopez LM, Grimes DA, Stockton LL, Gallo MF. Strategies to improve adherence and acceptability of hormonal methods of contraception. Cochrane Database of Systematic Reviews 2013, Issue 10. Art. No.: CD004317. DOI: 10.1002/14651858.CD004317.pub4.

123. Medicines and Healthcare products Regulatory Agency (MHRA). Combined Oral Contraceptives (The Pill): When to Start Taking the Pill, and Missed Pill Advice. MHRA UK Public Assessment Report, May 2011. [accesible online en www.mhra.gov.uk, accedido septiembre 2014].

124. Zapata LB, Steenland MW, Brahmi D, Marchbanks PA, Curtis KM. Patient understanding of oral contraceptive pill instructions related to missed pills: a systematic review. Contraception. 2013;87(5):674-84.

125. Deijen JB, Kornaat H. The influence of type of information, somatization, and locus of control on attitude, knowledge, and compliance with respect to the triphasic oral contraceptive Tri-Minulet. Contraception. 1997;56(1):31-41.

126. Little P, Griffin S, Kelly J, Dickson N, Sadler C. Effect of educational leaflets and questions on knowledge of contraception in women taking the combined contraceptive pill: randomised controlled trial. BMJ. 1998;316(7149):1948-52.

127. Chin-Quee D, Wong E, Cuthbertson C. Evaluating information on oral contraceptive use: a randomized controlled trial to assess missed pill instructions. Hum Reprod. 2006;21(12): 3137-45.

128. Hou MY, Hurwitz S, Kavanagh E, Fortin J, Goldberg AB. Using daily text-message reminders to improve adherence with oral contraceptives. Obstetrics and Gynecology 2010; 116(3): 633-40.

129. Castaño PM, Bynum JY, Andrés R, Lara M, Westhoff C. Effect of daily text messages on oral contraceptive continuation: a randomized controlled trial. Obstet Gynecol. 2012;119(1): 14-20.

130. Faculty of Family Planning and Reproductive Health Care. Drug Interactions with Hormonal Contraception (January 2011). Faculty of Sexual and Reproductive Healthcare - Clinical Effectiveness Unit, January 2011. [accesible online en www.fsrh.org/pdfs/CEUGuidanceDrugInteractionsHormonal.pdf, accedido 1 septiembre 2014].

131. Sidhu J, Job S, Philipson R. The pharmacokinetic and pharmacodynamic consequences of the co-administration of lamotrigine and a combined oral contraceptive in healthy female subjects. Br J Pharmacol 2005; 61: 191-199.

132. Comité d'Avaluació de Nous Medicaments. Ulipristal. Institut Català de la Salut - CANM número 7, Octubre 2010.

133. Faculty of Family Planning and Reproductive Health Care Clinical Effectiveness Unit. First prescription of combined oral contraception. J Fam Plann Reprod Health Care 2003;29:209_ 223.

134. Back DJ, Breckenridge AM, Crawford FE, Hall JM, MacIver M, Orme MLE, et al. The effect of rifampicin on the pharmokinetics of ethynylestradiol in women. Contraception 1980; 21: 135-143.

135. Barditch-Crovo P, Trapnell CB, Ette E, Zacur HA, Coresh J, Rocco LE, et al. The effects of rifampin and rifabutin on the pharmacokinetics and pharmacodynamics of a combination oral contraceptive. Clin Pharmacol Ther 1999; 65:428-438.

136. Gelbke HP, Gethmann U, Knuppen R. Influence of rifampicin treatment on the metabolic fate of [4-14C] mestranol in women. Horm Metab Res 1977;9:415-419.

137. Joshi JV, Joshi UM, Sankolli GM, Gupta K, Rao AP, Hazari K, et al. A study of interaction of a low-dose contraceptive with anti-tubercular drugs. Contraception 1980;21:617-629.

138. LeBel M, Masson E, Guilbert E, Colborn D, Paquet F, Allard S, et al. Effects of rifabutin and rifampicin on pharmacokinetics of ethinylestradiol and norethindrone. J Clin Pharmacol 1998;38:1042-1050. 
139. Abrams LS, Skee DM, Natarajan J, Hutman W, Wong FA. Tetracycline HCL does not affect the pharmakokinetics of a contraceptive patch. Int J Gynaecol Obstet 2000; 70(Suppl. 1): 57-58.

140. Back DJ, Grimmer SfM, Orme MLE, Proudlove C, Mann RD, Breckenridge AM. Evaluation of Committee on Safety of Medicines yellow card reports on oral contraceptive-drug interactions with anticonvulsants and antibiotics. Br J Clin Pharmacol 1988; 25: 527-532.

141. Bacon JF, Shenfield GM. Pregnancy attributable to interaction between tetracycline and oral contraceptives. BMJ 1980; 280: 293.

142. Bainton R. Interaction between antibiotic therapy and contraceptive medication. Oral Surg Oral Med Oral Pathol Oral Radiol Endod 1986; 61: 453-455.

143. Bollen M. Use of antibiotics when taking the oral contraceptive pill. Aust Fam Physician 1995; 24: 928-929.

144. Csemiczky G, Alvendal C, Landgren BM. Risk for ovulation in women taking a low-dose oral contraceptive (Microgynon) when receiving antibacterial treatment with fluoroquinolone (ofloxacin). Adv Contracept 1996; 12: 101-109.

145. DeSano EA, Hurley SC. Possible interactions of antihistamines and antibiotics with oral contraceptive effectiveness. Fertil Steril 1982; 37: 853-854.

146. Dogterom P, van den Heuvel MW, Thomsen T. Absence of pharmacokinetic interactions of the combined contraceptive vaginal ring Nuvaring with oral amoxicillin or doxycycline in two randomised trials. Clin Pharmacokinet 2005; 44: 429-438.

147. Donley TG, Smith RF, Roy B. Reduced oral contraceptive effectiveness with concurrent antibiotic use: a protocol for prescribing antibiotics to women of childbearing age. Compendium 1990; 11: 392-396.

148. Dossetor J. Drug interactions with oral contraceptives. BMJ 1984; 4: 467-468.

149. Maggiolo F, Puricelli G, Dottorini M, Caprioli S, Bianchi W, Suter F. The effect of ciprofloxacin on oral contraceptive steroid treatments. Drugs Exp Clin Res 1991; 17: 451-454.

150. Murphy AA, Zacur HA, Charace P, Burkman RT. The effect of tetracycline on level of oral contraceptives. Am J Obstet Gynecol 1991; 164: 28-33.

151. Neely JL, Abate M, Swinker M, D'Angio R. The effect of doxycycline on serum levels of ethinyl estradiol, norethindrone, and endogenous progesterone. Obstet Gynecol 1991; 77: 416-420.

152. Scholten PC, Droppert RM, Zwinkels MGL, Moesker HL, Nauta JJP, Hoepelman IM. No interaction between ciprofloxacin and an oral contraceptive. Antimicrob Agents Chemother 1998; 42: 3266-3268.

153. Silber TJ. Apparent oral contraceptive failure associated with antibiotic administration. J Adolesc Health Care 1983;4: 287-289.

154. Young LK, Farquhar CM, McCowan LME, Roberts HE, Taylor J. The contraceptive practices of women seeking termination of pregnancy in an Aukland clinic. N Z Med J 1994; 107 : 189-191.

155. Grimes DA, Lopez LM, Schulz KF. Antibiotic prophylaxis for intrauterine contraceptive device insertion. Cochrane Database of Systematic Reviews 1999, Issue 3. Art. No.: CD001327. DOI: 10.1002/14651858.CD001327.

156. Grimes DA, Schulz KF. Prophylactic antibiotics for intrauterine device insertion: a metaanalysis of the randomized controlled trials. Contraception. 1999;60(2):57-63.

157. Martínez F, López-Arregui E. Infection risk and intrauterine devices. Acta Obstet Gynecol Scand. 2009;88(3):246-50.

158. Sinei SK, Schulz KF, Lamptey PR, Grimes DA, Mati JK, Rosenthal SM, et al. Preventing IUCD-related pelvic infection: the efficacy of prophylactic doxycycline at insertion. $\mathrm{Br} \mathbf{J}$ Obstet Gynaecol. 1990;97(5):412-9. Walsh TL, Bernstein GS, Grimes DA, Frezieres R, 
Bernstein L, Coulson AH. Effect of prophylactic antibiotics on morbidity associated with IUD insertion: results of a pilot randomized controlled trial. IUD Study Group. Contraception. 1994;50(4):319-27.

159. Ladipo OA, Farr G, Otolorin E, Konje JC, Sturgen K, Cox P, et al. Prevention of IUD-related pelvic infection: the efficacy of prophylactic doxycycline at IUD insertion. Adv Contracept. 1991;7(1):43-54.

160. Zorlu CG, Aral K, Cobanoglu O, Gürler S, Gokmen O. Pelvic inflammatory disease and intrauterine devices: prophylactic antibiotics to reduce febrile complications. Adv Contracept. 1993;9(4):299-302.

161. Walsh T, Grimes D, Frezieres R, Nelson A, Bernstein L, Coulson A, et al. Randomised controlled trial of prophylactic antibiotics before insertion of intrauterine devices. IUD Study Group. Lancet. 1998;351(9108):1005-8.

162. Faculty of Family Planning and Reproductive Health Care. Recommendation from the CEU: Antibiotic prophylaxis for intrauterine contraceptive use in women at risk of bacterial endocarditis. Faculty of Sexual and Reproductive Healthcare - Clinical Effectiveness Unit, July 2008. [accesible online en www.fsrh.org/pdfs/CEUstatementBacterialEndocarditis.pdf, accedido septiembre 2012].

163. Caddy S, Yudin MH, Hakim J, Money DM; Infectious Disease Committee; Society of Obstetricians and Gynaecologists of Canada. Best practices to minimize risk of infection with intrauterine device insertion. J Obstet Gynaecol Can. 2014;36(3):266-76.

164. American Heart Association Rheumatic Fever, Endocarditis, and Kawasaki Disease Committee; American Heart Association Council on Cardiovascular Disease in the Young; American Heart Association Council on Clinical Cardiology; American Heart Association Council on Cardiovascular Surgery and Anesthesia; Quality of Care and Outcomes Research Interdisciplinary Working Group. Prevention of infective endocarditis: guidelines from the American Heart Association: a guideline from the American Heart Association Rheumatic Fever, Endocarditis, and Kawasaki Disease Committee, Council on Cardiovascular Disease in the Young, and the Council on Clinical Cardiology, Council on Cardiovascular Surgery and Anesthesia, and the Quality of Care and Outcomes Research Interdisciplinary Working Group. Circulation. 2007;116(15):1736-54.

165. National Institute for Health and Care Excellence. Prophylaxis against infective endocarditis: Antimicrobial prophylaxis against infective endocarditis in adults and children undergoing interventional procedures. NICE guidelines [CG64]. London, March 2008.

166. Allen RH, Bartz D, Grimes DA, Hubacher D, O'Brien P. Interventions for pain with intrauterine device insertion. Cochrane Database of Systematic Reviews 2009, Issue 3. Art. No.: CD007373. DOI: 10.1002/14651858.CD007373.pub2

167. Sääv I, Aronsson A, Marions L, Stephansson O, Gemzell-Danielsson K. Cervical priming with sublingual misoprostol prior to insertion of an intrauterine device in nulliparous women: a randomized controlled trial. Human Reproduction 2007;22: 2647-52.

168. Dijkhuizen K, Dekkers OM, Holleboom CA, de Groot CJ, Hellebrekers BW, van Roosmalen GJ, et al. Vaginal misoprostol prior to insertion of an intrauterine device: an RCT. Hum Reprod. 2011;26(2):323-9.

169. Edelman AB, Schaefer E, Olson A, Van Houten L, Bednarek P, Leclair C, et al. Effects of prophylactic misoprostol administration prior to intrauterine device insertion in nulliparous women. Contraception. 2011;84(3):234-9.

170. Scavuzzi A, Souza AS, Costa AA, Amorim MM. Misoprostol prior to inserting an intrauterine device in nulligravidas: a randomized clinical trial. Hum Reprod. 2013;28(8):2118-25.

171. Ibrahim ZM, Sayed Ahmed WA. Sublingual misoprostol prior to insertion of a T380A intrauterine device in women with no previous vaginal delivery. Eur J Contracept Reprod Health Care. 2013;18(4):300-8. 
172. Lathrop E, Haddad L, McWhorter CP, Goedken P. Self-administration of misoprostol prior to intrauterine device insertion among nulliparous women: a randomized controlled trial. Contraception. 2013;88(6):725-9.

173. Espey E, Singh RH, Leeman L, Ogburn T, Fowler K, Greene H. Misoprostol for intrauterine device insertion in nulliparous women: a randomized controlled trial. Am J Obstet Gynecol. 2014;210(3):208.e1-5.

174. Ward K, Jacobson JC, Turok DK, Murphy PA. A survey of provider experience with misoprostol to facilitate intrauterine device insertion in nulliparous women. Contraception 2011;84(6):594-9. doi: 10.1016/j.contraception.2011.03.011. Epub 2011 May 11.

175. Faculty of Family Planning and Reproductive Health Care. FSRH Clinical Guidance: Contraceptive Choices for Young People (March 2010). Faculty of Sexual and Reproductive Healthcare - Clinical Effectiveness Unit, March 2010. [accesible online en http://www.fsrh. org/standards-and-guidance/documents/cec-ceu-guidance-young-people-mar-2010/, accedido septiembre 2014].

176. Faculty of Family Planning and Reproductive Health Care. FSRH Clinical Guidance: Quick Starting Contraception (September 2010). Faculty of Sexual and Reproductive Healthcare Clinical Effectiveness Unit, September 2010. [accesible online en http://www.fsrh.org/standards-and-guidance/documents/cec-ceu-guidance-young-people-mar-2010/, accedido septiembre 2014].

177. Lara-Torre E, Schroeder B. Adolescent compliance and side effects with Quick Start initiation of oral contraceptive pills. Contraception. 2002;66(2):81-5.

178. Westhoff C, Kerns J, Morroni C, Cushman LF, Tiezzi L, Murphy PA. Quick start: novel oral contraceptive initiation method. Contraception. 2002;66(3):141-5.

179. Westhoff C, Morroni C, Kerns J, Murphy PA. Bleeding patterns after immediate vs. conventional oral contraceptive initiation: a randomized, controlled trial. Fertil Steril. 2003;79(2):322-9.

180. Edwards SM, Zieman M, Jones K, Diaz A, Robilotto C, Westhoff C. Initiation of oral contraceptives--start now! J Adolesc Health. 2008;43(5):432-6.

181. Lopez LM, Newmann SJ, Grimes DA, Nanda K, Schulz KF. Immediate start of hormonal contraceptives for contraception. Cochrane Database of Systematic Reviews 2012, Issue 12. Art. No.: CD006260. DOI: 10.1002/14651858.CD006260.pub3.

182. Murthy AS, Creinin MD, Harwood B, Schreiber CA. Same-day initiation of the transdermal hormonal delivery system (contraceptive patch) versus traditional initiation methods. Contraception. 2005;72(5):333-6.

183. Westhoff C, Osborne LM, Schafer JE, Morroni C Bleeding patterns after immediate initiation of an oral compared with a vaginal hormonal contraceptive. Obstet Gynecol. 2005;106(1):89-96.

184. Westhoff C, Heartwell S, Edwards S, Zieman M, Cushman L, Robilotto C, et al. Initiation of oral contraceptives using a quick start compared with a conventional start: a randomized controlled trial. Obstet Gynecol. 2007;109(6):1270-6.

185. Rickert VI, Tiezzi L, Lipshutz J, León J, Vaughan RD, Westhoff C. Depo Now: preventing unintended pregnancies among adolescents and young adults. J Adolesc Health. 2007;40(1): 22-8.

186. Deans EI, Grimes DA. Intrauterine devices for adolescents: a systematic review. Contraception. 2009;79(6):418-23.

187. Secura GM, Madden T, McNicholas C, Mullersman J, Buckel CM, Zhao Q, et al. Provision of no-cost, long-acting contraception and teenage pregnancy. N Engl J Med. 2014;371(14): 1316-23.

188. Jorgensen V. One-year contraceptive follow-up of adolescent patients. Am J Obstet Gynecol 1973;115:484-6. 
189. Goldman JA, Dekel A, Reichman J. Immediate post abortion intrauterine contraception in nulliparous adolescents. Isr J Med Sci 1979;15:522-5.

190. Goldman JA, Reichman J. Contraception in the teenager. A comparison of four methods of contraception in adolescent girls. Isr J Med Sci 1980;16:510-3.

191. Diaz J, Pinto Neto AM, Bahamondes L, Diaz M, Arce XE, Castro S. Performance of the copper T 200 in parous adolescents: are copper IUDs suitable for these women? Contraception 1993;48:23-8.

192. Lane ME, Sobrero AJ. Experience with intrauterine contraception by adolescent women. Mt Sinai J Med 1975;42:337-44.

193. Weiner E, Berg AA, Johansson I. Copper intrauterine contraceptive devices in adolescent nulliparae. Br J ObstetGynaecol 1978;85:204-6.

194. Kulig JW, Rauh JL, Burket RL, Cabot HM, Brookman RR. Experience with the copper 7 intrauterine device in an adolescent population. J Pediatr 1980;96:746-50.

195. Larsson B, Hagstrom B, Viberg L, Hamberger L. Long-term clinical experience with the Cu7-IUD. Evaluation of a prospective study. Contraception 1981;23:387-97.

196. Rosenstock JR, Peipert JF, Madden T, Zhao Q, Secura GM. Continuation of reversible contraception in teenagers and young women. Obstet Gynecol. 2012;120(6):1298-305.

197. Paterson H, Ashton J, Harrison-Woolrych M.A nationwide cohort study of the use of the levonorgestrel intrauterine device in New Zealand adolescents. Contraception. 2009;79(6): 433-8.

198. Luukkainen T, Allonen H, Haukkamaa M, Holma P, Pyörälä T, Terho J, et al. Effective contraception with the levonorgestrel-releasing intrauterine device: 12-month report of a European multicenter study. Contraception. 1987;36(2):169-79.

199. Rivera R, Chen-Mok M, McMullen S. Analysis of client characteristics that may affect early discontinuation of the TCu-380A IUD. Contraception. 1999;60(3):155-60.

200. Diedrich JT, Desai S, Zhao Q, Secura G, Madden T, Peipert JF. Association of short-term bleeding and cramping patterns with long-acting reversible contraceptive method satisfaction. Am J Obstet Gynecol. 2015;212(1):50.e1-8.

201. Thonneau P, Almont T, de La Rochebrochard E, Maria B. Risk factors for IUD failure: results of a large multicentre case-control study. Hum Reprod. 2006;21(10):2612-6.

202. Madden T, McNicholas C, Zhao Q, Secura GM, Eisenberg DL, Peipert JF. Association of age and parity with intrauterine device expulsion. Obstet Gynecol. 2014;124(4):718-26.

203. Centers for Disease Control and Prevention (CDC). Sexually Transmitted Disease Surveillance 2013. Atlanta: U.S. Department of Health and Human Services; 2014.

204. Centro Nacional de Epidemiología. Resultados de la vigilancia epidemiológica de las enfermedades transmisibles. Informe anual 2012. Madrid, 2014.

205. Centre d'Estudis Epidemiològics sobre les Infeccions de Transmissió Sexual i Sida de Catalunya (CEEISCAT). Informe epidemiològic CEEISCAT. Badalona: 2015, Generalitat de Catalunya. Agència de Salut Pública de Catalunya.

206. Hov GG, Skjeldestad FE, Hilstad T. Use of IUD and subsequent fertility--follow-up after participation in a randomized clinical trial. Contraception. 2007;75(2):88-92.

207. Mestad R, Secura G, Allsworth JE, Madden T, Zhao Q, Peipert JF. Acceptance of long-acting reversible contraceptive methods by adolescent participants in the Contraceptive CHOICE Project. Contraception. 2011;84(5):493-8.

208. Eisenberg D, McNicholas C, Peipert JF. Cost as a barrier to long-acting reversible contraceptive (LARC) use in adolescents. J Adolesc Health. 2013;52(4 Suppl):S59-63.

209. Udeh, B; Losch, M; Spies, E (2009), The Cost of Unintended Pregnancy in Iowa: A BenefitCost Analysis of Public Funded Family Planning Services, The University of Iowa Public Policy Center. 
210. American College of Obstetricians and Gynecologists Committee on Gynecologic Practice; Practice Committee of the American Society for Reproductive Medicine. Female age-related fertility decline. Committee Opinion No. 589. Obstet Gynecol. 2014r;123(3):719-21.

211. Fretts RC, Wilknins-Haug L, Eckler K. Effect of advanced age on fertility and pregnancy in women. Uptodate. Nov 2014.

212. Reynolds RF, Obermeyer CM. Age at natural menopause in Spain and the United States: results from the DAMES project. Am J Hum Biol. 2005;17(3):331-40.

213. Bernis C, Reher DS. Environmental contexts of menopause in Spain: comparative results from recent research. Menopause. 2007;14(4):777-87.

214. Instituto Nacional de Estadística. Encuesta de fecundidad. Año 1999. Consultada en línea en http://www.ine.es/inebmenu/mnu_dinamicapob.htm

215. Instituto Nacional de Estadística. Nacimientos ocurridos en España. Año 2013. Consultada en línea en http://www.ine.es/inebmenu/mnu_dinamicapob.htm

216. Balasch J, Gratacós E. Delayed childbearing: effects on fertility and the outcome of pregnancy. Curr Opin Obstet Gynecol. 2012;24(3):187-93.

217. Collaborative Group on Hormonal Factors in Breast Cancer. Breast cancer and hormonal contraceptives: collaborative reanalysis of individual data on 53297 women with breast cancer and 100239 women without breast cancer from 54 epidemiological studies. Lancet. 1996;347(9017):1713-27.

218. Faculty of Sexual and Reproductive Healthcare. Clinical Effectiveness Unit. Contraception for Women Aged Over 40 Years. FSRH Guidance (July 2010).

219. Faculty of Sexual and Reproductive Healthcare. Clinical Effectiveness Unit. Intrauterine Contraception. FSRH Guidance (November 2007).

220. Bahamondes L, Faundes A, Sobreira-Lima B, Lui-Filho JF, Pecci P, Matera S. TCu 380A IUD: a reversible permanent contraceptive method in women over 35 years of age. Contraception. 2005;72(5):337-41.

221. NICE CG 30: National Collaborating Centre for Women's and Children's Health. Long-acting reversible contraception: the effective and appropriate use of long-acting reversible contraception. National Institute for Health and Clinical Excellence, October 2005 (updated 2011).

222. RCOG 40 Royal College of Obstetricians and Gynaecologists. Venous Thromboembolism and Hormonal Contraception (Green-Top 40). July 2010.

223. Trussell J, Guthrie KA, Schwarz EB. Much ado about little: obesity, combined hormonal contraceptive use and venous thrombosis. Contraception. 2008;77(3):143-6.

224. Weisberg E. Contraceptive options for women in selected circumstances. Best Pract Res Clin Obstet Gynaecol. 2010;24(5):593-604.

225. Society of Family Planning, Higginbotham S. Contraceptive considerations in obese women: release date 1 September 2009, SFP Guideline 20091. Contraception. 2009;80(6):583-90.

226. Lopez LM, Grimes DA, Chen M, Otterness C, Westhoff C, Edelman A, Helmerhorst FM. Hormonal contraceptives for contraception in overweight or obese women. Cochrane Database of Systematic Reviews 2013, Issue 4. Art. No.: CD008452. DOI:10.1002/14651858. CD008452.pub3.

227. Burkman RT, Fisher AC, Wan GJ, Barnowski CE, LaGuardia KD. Association between efficacy and body weight or body mass index for two low-dose oral contraceptives. Contraception 2009;79(6):424-7.

228. Merki-Feld GS, Skouby S, Serfaty D, Lech M, Bitzer J, Crosignani PG, et al. European society of contraception statement on contraception in obese women. Eur J Contracept Reprod Health Care. 2015 Feb;20(1):19-28. 
229. Xu H, Wade JA, Peipert JF, Zhao Q, Madden T, Secura GM. Contraceptive failure rates of etonogestrel subdermal implants in overweight and obese women. Obstet Gynecol. 2012;120 (1):21-6.

230. Abdollahi M, Cushman M, Rosendaal F. Obesity: risk of venous thrombosis and the interaction with coagulation factor levels and oral contraceptive use. Thrombosis \& Haemostasis 2003;89:493-8.

231. Lidegaard O, Edstrom B, Kreiner S. Oral contraceptives and venous thromboembolism: a five-year national case-control study. Contraception 2002;65:187-96.

232. Nightingale AL, Lawrenson RA, Simpson EL, Williams TJ, MacRae KD, Farmer RD. The effects of age, body mass index, smoking and general health on the risk of venous thromboembolism in users of combined oral contraceptives. Eur J Contracept Reprod Health Care 2000;5:265-74.

233. Pomp ER, le Cessie S, Rosendaal FR, Doggen CJ. Risk of venous thrombosis: obesity and its joint effect with oral contraceptive use and prothrombotic mutations. Br J Haematol 2007;139:289-96.

234. Sidney S, Petitti DB, Soff GA, Cundiff DL, Tolan KK, Quesenberry Jr CP. Venous thromboembolic disease in users of low-estrogen combined estrogen-progestin oral contraceptives. Contraception 2004;70:3-10.

235. Kaneshiro B, Jensen JT, Carlson NE, Harvey SM, Nichols MD, Edelman AB. Body mass index and sexual behavior. Obstet Gynecol 2008;112:586-92

236. Chuang CH, Chase GA, Bensyl DM, Weisman CS. Contraceptive use by diabetic and obese women. Women's Health Issues 2005;15:167.

237. Heinemann LA, Dinger JC. Range of published estimates of venous thromboembolism incidence in young women. Contraception. 2007;75(5):328-36.

238. Zieman M, Guillebaud J, Weisberg E, Shangold GA, Fisher AC, Creasy GW. Contraceptive efficacy and cycle control with the Ortho Evra/Evra transdermal system: the analysis of pooled data. Fertil Steril. 2002;77(2 Suppl 2):S13-8.

239. Jain J, Jakimiuk AJ, Bode FR, Ross D, Kaunitz AM. Contraceptive efficacy and safety of DMPA-SC. Contraception. 2004;70(4):269-75.

240. Vilos GA, Marks J, Tureanu V, Abu-Rafea B, Vilos AG. The levonorgestrel intrauterine system is an effective treatment in selected obese women with abnormal uterine bleeding. $\mathbf{J}$ Minim Invasive Gynecol. 2011;18(1):75-80.

241. Callegari LS, Nelson KM, Arterburn DE, Prager SW, Schiff MA, Schwarz EB. Factors associated with lack of effective contraception among obese women in the United States. Contraception. 2014;90(3):265-71.

242. Grupo de trabajo de la Guía de práctica clínica de atención en el embarazo y puerperio. Guía de práctica clínica de atención en el embarazo y puerperio. Ministerio de Sanidad, Servicios Sociales e Igualdad. Agencia de Evaluación de Tecnologías Sanitarias de Andalucía; 2014. Guías de Práctica Clínica en el SNS: AETSA 2011/10.

243. Jackson E, Glasier A. Return of ovulation and menses in postpartum nonlactating women: a systematic review. Obstet Gynecol. 2011;117(3):657-62.

244. Faculty of Sexual and Reproductive Healthcare. Postnatal Sexual and Reproductive Health. FSRH Guidance (2009).

245. Jackson E, Curtis KM, Gaffield ME. Risk of venous thromboembolism during the postpartum period: a systematic review. Obstet Gynecol. 2011;117(3):691-703.

246. Kapp N, Curtis K, Nanda K. Progestogen-only contraceptive use among breastfeeding women: a systematic review. Contraception. 2010;82(1):17-37. 
247. Van der Wijden C, Brown J, Kleijnen J. Lactational amenorrhea for family planning. Cochrane Database of Systematic Reviews 2003, Issue 4. Art. No.: CD001329. DOI:10.1002/14651858. CD001329.

248. Heinemann K, Reed S, Moehner S, Minh TD. Risk of uterine perforation with levonorgestrel-releasing and copper intrauterine devices in the European Active Surveillance Study on Intrauterine Devices. Contraception. 2015;91(4):274-9.

249. AEMPS Agencia Española de Medicamentos y Productos Sanitarios. Comunicación directa para profesionales sanitarios. Información actualizada acerca del riesgo de perforación uterina con dispositivos intrauterinos de cobre (Nova T 380) y sistemas de liberación intrauterinos de levonorgestrel (Mirena y Jaydess). Octubre 2015. Cartas de seguridad a los profesionales sanitarios. En: www.aemps.gob.es/vigilancia/medicamentosUsoHumano/cartas_segProfSani. htm

250. Joint Formulary Committee. British National Formulary (BNF 59). 2010.

251. Stockley IH. Stockley's Drug Interactions (9th edn). London, UK: Pharmaceutical Press, 2010.

252. Cole JA, Norman H, Doherthy M, Walker AM. Venous thromboembolism, myocardial infarction, and stroke among transdermal contraceptive system users. Obstet Gynecol 2007;109:339-46. 
